МИНИСТЕРСТВО ОБРАЗОВАНИЯ И НАУКИ, МОЛОДЕЖИ И СПОРТА УКРАИНЫ

ДНЕПРОПЕТРОВСКИЙ НАЦИОНАЛЬНЫЙ УНИВЕРСИТЕТ ЖЕЛЕЗНОДОРОЖНОГО ТРАНСПОРТА

ИМЕНИ АКАДЕМИКА В. ЛАЗАРЯНА

\title{
Физические зависимости упругих массивов
}

МОНОГРАФИЯ

ИЗДАТЕЛЬСТВО

ДНЕПРОПЕТРОВСКОГО НАЦИОНАЛЬНОГО УНИВЕРСИТЕТА ЖЕЛЕЗНОДОРОЖНОГО ТРАНСПОРТА ИМЕНИ АКАДЕМИКА В. ЛАЗАРЯНА 2012 
Автор:
канд. техн. наук, доц. И. К. Бадалаха.

Рецензенты: д-р техн. наук, проф. Н. Н. Беляев, д-р техн. наук, проф. Е. А. Сдвижкова, д-р техн. наук, проф. С. З. Полищук.

Печатается по решению ученого совета Днепропетровского национального университета железнодорожного транспорта имени академика В. Лазаряна (протокол № 13 от 29.06.11)

\section{УДК 624.044}

Физические зависимости упругих массивов [Текст]: монография / И. К. Бадалаха. - Д.: Изд-во Днепропетр. нац. ун-та ж.-д. трансп. им. акад. В. Лазаряна, 2012. - 197 с. ISBN 978-966-8471-51-3

Деформации упругой среды делятся на два вида соответственно их происхождению: объемные и чистого формоизменения. Каждый вид деформации подчиняется своей, отдельной, зависимости, поэтому они определяются раздельно.

Результаты выполненной работы могут быть использованы специалистами в области физики и механики деформируемых твердых тел, в том числе грунтов, преподавателями, аспирантами и студентами высших учебных заведений, проектировщиками при расчете оснований сооружений.

Ил. 41. Табл. 3. Біблиогр.: 29 наим.

Deformations of the elastic environment divide into two kinds according to their origin: extensional and of a pure form changing. Each kind of deformation is under it's individual dependence, therefore they are determined separately.

Executed job performances can be used by the specialists in the field of physics and mechanics of the deformed solids, including soils, by teachers, post-graduate students and students of higher educational establishments, by the designers at the calculation of building grounds.

ISBN 978-966-8471-51-3

(C) И. К. Бадалаха, 2012

(C) Изд-во Днепропетр. нац. ун-та ж.-д. трансп. им. акад. В. Лазаряна, оригиналмакет, 2012 
Моей alma mater - родному ДИИТу посвящุаю... 



\section{СОДЕРЖАНИЕ}

ПРЕДИСЛОВИЕ

ОСНОВНЫЕ ПОЛОЖЕНИЯ СОВРЕМЕННОЙ ТЕОРИИ УПРУГОСТИ............ 8

1.1. Зависимости напряженного состояния в упругом массиве ........................... 8

1.2. Зависимости деформированного состояния в упругом массиве ..................... 14

1.3. Зависимости компонентов деформаций от компонентов перемещений ....... 17

1.4. Физические зависимости в линейно-упругой изотропной среде ...................20

1.5. Плоское деформирование упругой среды...............................................26

АНАЛИЗ НАПРЯЖЕННО-ДЕФОРМИРОВАННОГО СОСТОЯНИЯ УПРУГИХ МАССИВОВ В РЕШЕНИИ ТЕОРИИ УПРУГОСТИ........................ 30

2.1. Решение Буссинеска ..................................................................................... 30

2.2. Решение Фламана ........................................................................................... 48

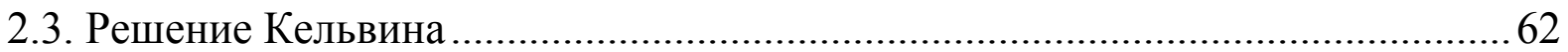

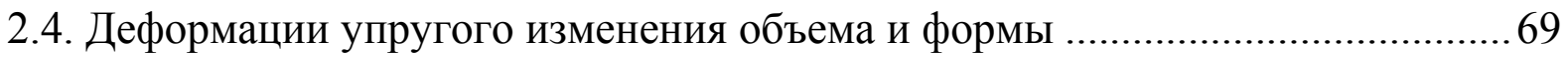

Выводы по главе 2 ................................................................................... 80

ВЫБОР ФИЗИЧЕСКИХ ЗАВИСИМОСТЕЙ УПРУГИХ ТЕЛ ...........................82

3.1. Метод аналогий в физических явлениях...................................................... 82

3.2. Зависимости объемного деформирования ................................................... 88

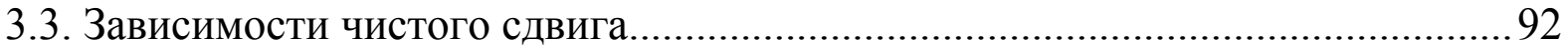

3.4. Оценка возможности независимого существования двух видов

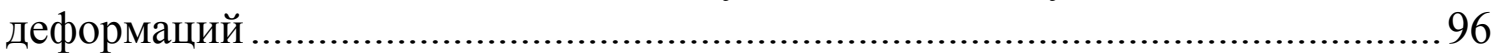

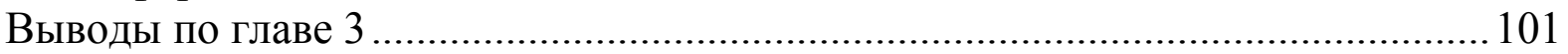

ЗАДАЧИ, РЕШАЕМЫЕ С ПОМОЩЬЮ ПРЕДЛОЖЕННЫХ

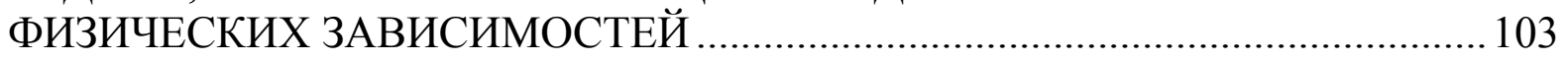

4.1. Напряженно-деформированное состояние упругого изотропного полупространства при различных вариантах его нагружения

4.1.1. Сосредоточенная сила, приложенная по нормали к поверхности полупространства

4.1.2. Сосредоточенная сила, приложенная по касательной к поверхности полупространства .

4.1.3. Сосредоточенная сила, приложенная наклонно к поверхности полупространства.

4.1.4. Нормальная нагрузка, равномерно распределенная вдоль линий конечной и бесконечной длины.

4.1.5. Нормальная нагрузка, равномерно распределенная на полосе постоянной ширины конечной и бесконечной длины

4.1.6. Касательная нагрузка, равномерно распределенная на поверхности полупространства вдоль линий конечной и бесконечной длины. 
4.1.7. Наклонная нагрузка, равномерно распределенная на поверхности полупространства вдоль линий конечной и бесконечной длины.

4.2. Напряженно-деформированное состояние упругого изотропного пространства от действия сосредоточенной внутри его силы.

4.3. Плоская деформация бесконечно длинных упругих массивов конечной толщины с ограниченной и неограниченной шириной.

4.3.1. Упругий массив ограниченной ширины на жестком основании, допускающем только горизонтальные смещения

4.3.2. Упругий массив ограниченной ширины на жестком основании, допускающем только вертикальные смещения

4.3.3. Упругий массив ограниченной ширины на жестком основании, допускающем частично горизонтальные и вертикальные смещения

4.3.4. Бесконечно простирающийся упругий массив на жестком основании, допускающем только горизонтальные смещения

4.3.5. Бесконечно простирающийся упругий массив на жестком основании, допускающем только вертикальные смещения

4.3.6. Бесконечно простирающийся упругий массив на жестком основании, допускающем частично горизонтальные и вертикальные смещения 


\section{ПРЕДИС ЛОВ ИЕ}

В современной теории упругости используются физические зависимости, отражающие связь между напряженным и деформированными состояниями упругой среды, предложенные Коши в 1822 году и впоследствии названные обобщенным законом Гука. Эти зависимости в общем носят феноменологический характер: в них деформации не разделяются по причинам происхождений, а подчиняются одной общей закономерности, поэтому их применение приводит к серьезным недостаткам в решении задач по определению напряженнодеформированного состояния упругой среды. Такие зависимости были приемлемы в науке того времени, поскольку ее уровень не позволял дать теоретическое обоснование для более сложных физических зависимостей. Такая возможность, в принципе, появилась лишь после нахождения формулы Остроградского (1828 г.), выражающей зависимость потока векторного поля через малую замкнутую поверхность с объемом, очерченным этой поверхностью, и формулы Стокса (1854 г.), связывающей циркуляцию векторного поля по замкнутому контуру с потоком ротора этого поля через поверхность, ограниченную указанным контуром. Однако такая возможность пока не была реализована.

Настоящая работа посвящена поиску и обоснованию таких физических зависимостей, применение которых в решениях задач о напряженно-деформированном состоянии упругих массивов не приводило бы к нарушениям общепринятых в мировой науке физических законов и положений.

Уважаемые читатели, автор будет благодарен, если вы направите ему свои замечания и отзывы по данной работе электронной почтой: badalaha@rambler.ru. 
ГЛАВА 1

\section{Основные положения \\ современной теории упругости}

\section{1. Зависимости напряженного состояния в упругом массиве}

Если вблизи произвольной точки упругого массива $M(x, y, z)$ провести три плоскости, параллельные координатным осям, и пересечь их наклонной к ним плоскостью, то получим элементарный тетраэдр (рис. 1.1). Действующие по его граням полные напряжения принято обозначать так [1]: $p_{x}, p_{y}, p_{z}, p_{v}$.

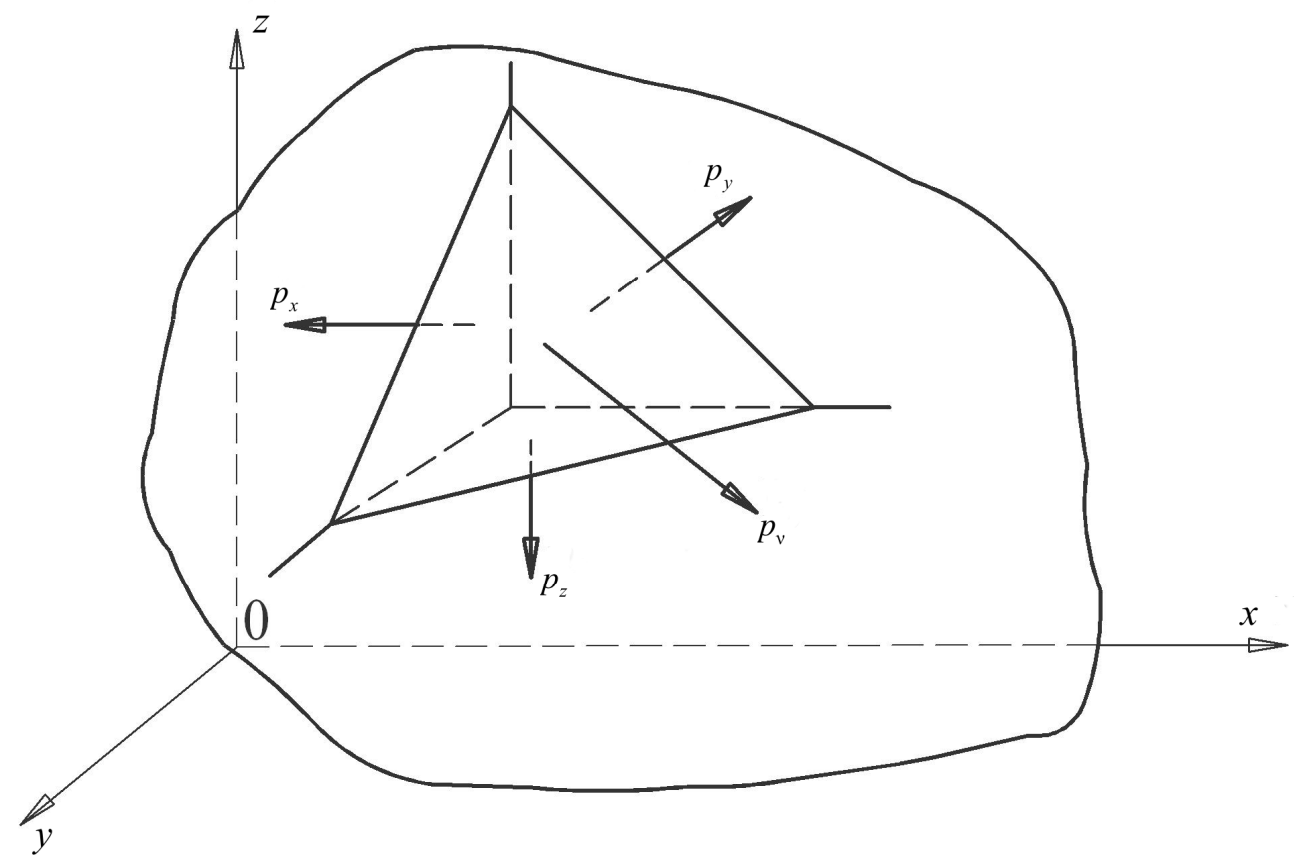

Рис. 1.1. Обозначение полных напряжений на гранях тетраэдра, выделенного возле заданной точки 
Составляющие первых трех напряжений, параллельные координатным осям, действуют по трем взаимно ортогональным площадкам, параллельным координатным, соответственно yoz, xoz, xoy и обозначаются:

от $p_{x}$ - составляющие $p_{x x} ; p_{y x} ; p_{z x}$;

от $p_{y}$ - составляющие $p_{x y} ; p_{y y} ; p_{z y}$;

от $p_{z}$ - составляющие $p_{x z} ; p_{y z} ; p_{z z}$.

Составляющие $p_{x x}, p_{y y}, p_{z z}$, действующие по нормалям к площадкам, называют нормальными напряжениями, остальные - касательными. В дальнейшем нормальные напряжения будем обозначать буквой $\sigma$ с приданием ей индекса, указывающего ось, параллельно которой направлено это напряжение. Для касательных напряжений используем букву $\tau$ с двумя индексами: $\tau_{x y}$ и $\tau_{y x} ; \tau_{y z}$ и $\tau_{z y} ; \tau_{z x}$ и $\tau_{x z}$, первый из которых обозначает направление действия данного напряжения, а второй - направление нормали к площадке его действия.

На рис. 1.2 показаны обозначения компонентов напряжений по трем видимым граням бесконечно малого параллелепипеда, который мысленно выделен в заданной точке массива. По трем невидимым граням соответствующие напряжения следует направлять в противоположную сторону. При этом знаки напряжений определяются с учетом нижеизложенных правил.

Нормальное напряжение считается положительным, когда оно вызывает растяжение в рассматриваемом элементе, и отрицательным, когда приводит к его сжатию.

За положительное направление касательного напряжения принимается положительное направление параллельной ему координатной оси, если растягивающее нормальное напряжение по этой же площадке имеет направление, совпадающее с положительным направлением той оси, параллельно которой оно действует. Если же растягивающее напряжение на этой площадке имеет направление, противоположное положительному направлению соответствующей координатной оси, то за положительное направление компонента касательного напряжения по той же площадке принимается отрицательное направление параллельной ему оси координат.

Если размеры выделенного параллелепипеда бесконечно малы, то одноименные и параллельные напряжения для каждых двух параллельных граней будут практически одинаковыми. 
В том случае когда наклонная к координатным плоскостям площадка является главной (см. рис. 1.1), то для нее $\tau=0$, поэтому $\sigma_{v}=p_{v}$, т. е. полное и нормальное напряжения для главной площадки совпадают по величине и направлению.

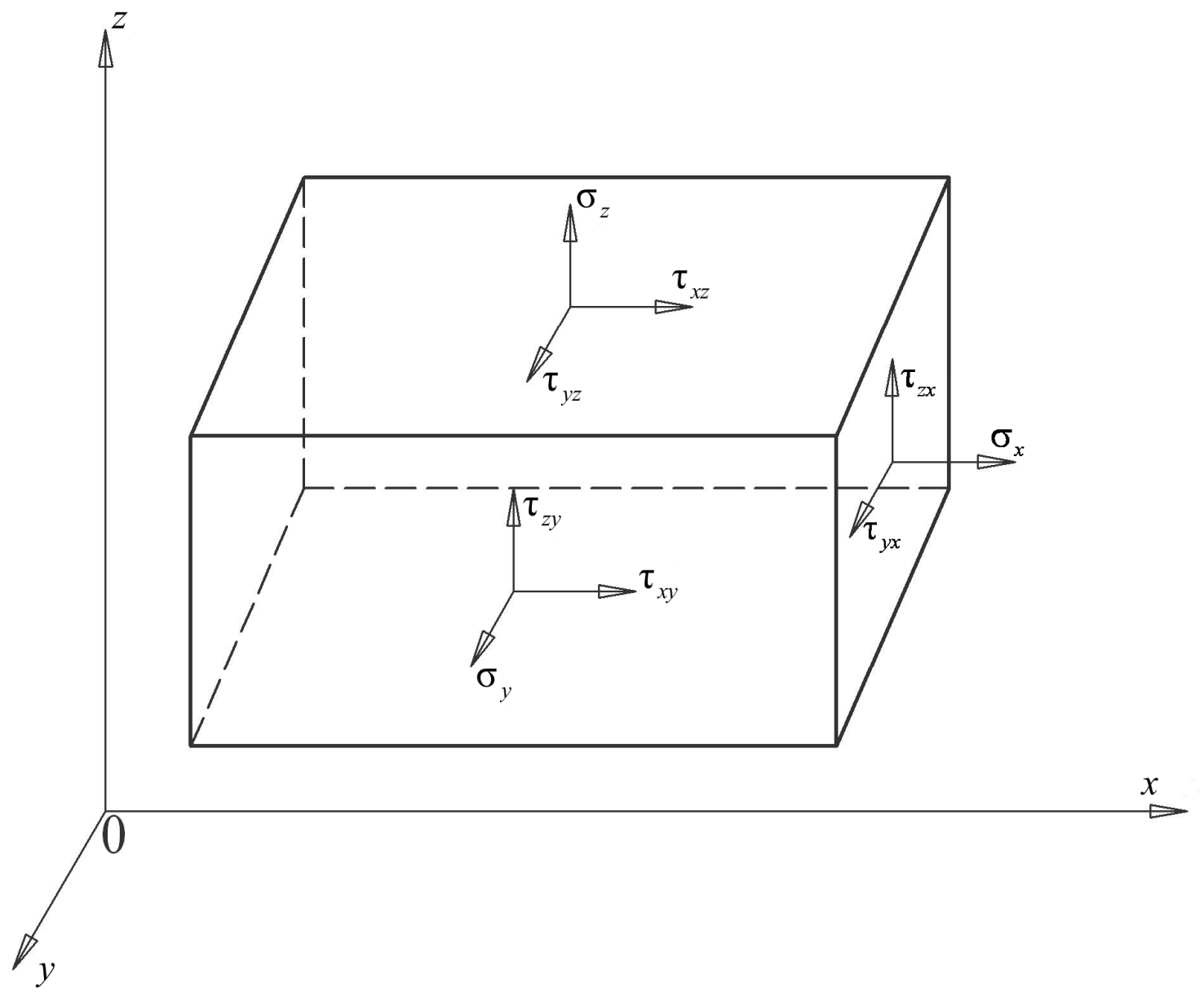

Рис. 1.2. Обозначение компонентов напряжений на гранях бесконечно малого параллелепипеда

В общем случае пространственно напряженного состояния массива через каждую его точку всегда можно провести три взаимно ортогональные плоскости, на которых будут отсутствовать касательные напряжения, а действующие на них нормальные напряжения имеют стационарные, т. е. независимые от координат, значения, называемые главными.

В целях нахождения главных напряжений для произвольной точки упругого массива можно составить кубическое уравнение, связыва- 
ющее величину главных напряжений в выбранной системе координат:

$$
\begin{aligned}
\sigma^{3}-\sigma^{2}\left(\sigma_{x}+\sigma_{y}+\sigma_{z}\right) & +\sigma\left(\sigma_{x} \sigma_{y}+\sigma_{y} \sigma_{z}+\sigma_{z} \sigma_{x}-\tau_{x y}^{2}-\tau_{y z}^{2}-\tau_{z x}^{2}\right)- \\
& -\left(\sigma_{x} \sigma_{y} \sigma_{z}+2 \tau_{x y} \tau_{y z} \tau_{z x}-\sigma_{x} \tau_{y z}^{2}-\sigma_{y} \tau_{z x}^{2}-\sigma_{z} \tau_{x y}^{2}\right)=0 .
\end{aligned}
$$

Решение уравнения (1.1) даст три действительных корня для определения главных напряжений ( $\sigma_{1}-$ максимальное значение, $\sigma_{2}-$ минимальное значение, $\sigma_{3}$ - промежуточное значение). Корни этого уравнения не зависят от системы координат, поэтому и коэффициенты его также не зависят от выбора координатной системы, т. е. эти коэффициенты являются инвариантами преобразования координат.

В связи с этим уравнение (1.1) можно записать в виде

$$
\sigma^{3}-\sigma^{I} \sigma^{2}+\sigma^{I I} \sigma-\sigma^{I I I}=0
$$

где $\sigma^{I}, \sigma^{I I}$ и $\sigma^{I I I}$ - инвариантные соотношения, называемые соответственно первым, вторым и третьим инвариантами напряженного состояния.

Первый, линейный инвариант представляет собой сумму нормальных напряжений по любым трем ортогональным площадкам в данной точке. В дальнейшем будем обозначать его $I_{1}$.

Напряженное состояние для элементарного объема можно записать в виде матрицы

$$
T_{\mathrm{H}}=\left\{\begin{array}{ccc}
\sigma_{x}, & \tau_{x y}, & \tau_{x z} ; \\
\tau_{y x}, & \sigma_{y}, & \tau_{y z} \\
\tau_{z x}, & \tau_{z y}, & \sigma_{z}
\end{array}\right\}
$$

которую называют тензором напряжения.

Для суждения о прочности упругого материала иногда целесообразно выделить те компоненты, которые связаны с изменением объема, и отдельно рассмотреть компоненты деформации, имеющие отношение к изменению формы. С этой целью вводится обозначение 


$$
\sigma_{\mathrm{cp}}=\frac{1}{3}\left(\sigma_{x}+\sigma_{y}+\sigma_{z}\right)=\frac{1}{3} \sigma^{I}
$$

а тензор (1.2) представляется в виде двух составляющих $T_{\mathrm{H}}=T_{\mathrm{H}}^{0}+D_{\text {н }}$, первую из которых

$$
T_{\mathrm{H}}^{0}=\left\{\begin{array}{ccc}
\sigma_{\mathrm{cp}}, & 0, & 0 \\
0, & \sigma_{\mathrm{cp}}, & 0 \\
0, & 0, & \sigma_{\mathrm{cp}}
\end{array}\right\}
$$

называют шаровой тензор напряжений, а вторую

$$
D_{\text {н }}=\left\{\begin{array}{ccc}
\left(\sigma_{\mathrm{cp}}-\sigma_{x}\right), & \tau_{x y}, & \tau_{x z} ; \\
\tau_{y x}, & \left(\sigma_{\mathrm{cp}}-\sigma_{y}\right), & \tau_{y z} ; \\
\tau_{z y}, & \tau_{z y}, & \left(\sigma_{\mathrm{cp}}-\sigma_{z}\right)
\end{array}\right\}
$$

девиатор напряжений.

Если упругое тело после приложения нагрузки находится в равновесии, то для любого выделенного из него элементарного куба должны удовлетворяться шесть условий равновесия (здесь рассматривается статическое равновесие тела):

$$
\begin{array}{ll}
\sum x=0 ; & \sum M_{x}=0 ; \\
\sum y=0 ; & \sum M_{y}=0 ; \\
\sum z=0 ; & \sum M_{z}=0 .
\end{array}
$$

После преобразований получаем систему дифференциальных статических уравнений равновесия Коши без инерционных сил: 


$$
\left.\begin{array}{c}
\frac{\partial \sigma_{x}}{\partial x}+\frac{\partial \tau_{x y}}{\partial y}+\frac{\partial \tau_{x z}}{\partial z}=0 \\
\frac{\partial \tau_{y x}}{\partial x}+\frac{\partial \sigma_{y}}{\partial y}+\frac{\partial \tau_{y z}}{\partial z}=0 \\
\frac{\partial \tau_{z x}}{\partial x}+\frac{\partial \tau_{z y}}{\partial y}+\frac{\partial \sigma_{z}}{\partial z}=0
\end{array}\right\}
$$

В каждой строке системы уравнений (1.3) содержится условие равенства нулю проекций действующих на элемент сил на ось координат - соответственно $x, y, z$. Как видим, эта система не содержит условий равенства нулю действующих моментов относительно тех же осей, однако из них вытекает закон парности касательных напряжений:

$$
\left.\begin{array}{l}
\tau_{x y}=\tau_{y x} ; \\
\tau_{z x}=\tau_{x z} ; \\
\tau_{y z}=\tau_{z y} .
\end{array}\right\}
$$

Таким образом, количество неизвестных компонентов напряжений сокращается с девяти до шести.

Если через одну и ту же точку упругой среды с прямоугольными координатами $x, y, z$ провести новую систему прямоугольных координат $x^{\prime}, y^{\prime}, z^{\prime}$, то то же самое напряженное состояние будет определяться шестью новыми компонентами напряжений на трех взаимно перпендикулярных элементарных площадках, проходящих через данную точку нормально новым осям:

$$
\sigma_{x^{\prime}}^{\prime} ; \sigma_{y^{\prime}}^{\prime} ; \sigma_{z^{\prime}}^{\prime} ; \tau_{x^{\prime} y^{\prime}}^{\prime}\left(=\tau_{y^{\prime} x^{\prime}}^{\prime}\right) ; \tau_{x^{\prime} z^{\prime}}^{\prime}\left(=\tau_{z^{\prime} x^{\prime}}^{\prime}\right) ; \tau_{y^{\prime} z^{\prime}}^{\prime}\left(=\tau_{z^{\prime} y^{\prime}}^{\prime}\right) \text {. }
$$

Эти шесть новых компонентов выражаются через шесть прежних компонентов

$$
\sigma_{x} ; \sigma_{y} ; \sigma_{z} ; \tau_{x y}\left(=\tau_{y x}\right) ; \tau_{x z}\left(=\tau_{z x}\right) ; \tau_{y z}\left(=\tau_{z y}\right)
$$

зависимостями [2]: 


$$
\begin{aligned}
& \sigma_{x^{\prime}}^{\prime}=l_{1}^{2} \sigma_{x}+m_{1}^{2} \sigma_{y}+n_{1}^{2} \sigma_{z}+2 m_{1} n_{1} \tau_{z y}+2 n_{1} l_{1} \tau_{z x}+2 m_{1} l_{1} \tau_{x y} ; \\
& \tau_{y^{\prime} x^{\prime}}^{\prime}=l_{1} l_{2} \sigma_{x}+m_{1} m_{2} \sigma_{y}+n_{1} n_{2} \sigma_{z}+\left(m_{1} n_{2}+m_{2} n_{1}\right) \tau_{y z}+\left(n_{1} l_{2}+n_{2} l_{1}\right) \tau_{z x}+ \\
& +\left(l_{1} m_{2}+l_{2} m_{1}\right) \tau_{x y} \\
& \tau_{z^{\prime} x^{\prime}}^{\prime}=l_{1} l_{3} \sigma_{x}+m_{1} m_{3} \sigma_{y}+n_{1} n_{3} \sigma_{z}+\left(m_{1} n_{3}+m_{3} n_{1}\right) \tau_{y z}+\left(n_{1} l_{3}+n_{3} l_{1}\right) \tau_{z x}+ \\
& +\left(l_{3} m_{1}+l_{1} m_{3}\right) \tau_{x y} \\
& \sigma_{y^{\prime}}^{\prime}=l_{2}^{2} \sigma_{x}+m_{2}^{2} \sigma_{y}+n_{2}^{2} \sigma_{z}+2 m_{2} n_{2} \tau_{z y}+2 n_{2} l_{2} \tau_{z x}+2 m_{2} l_{2} \tau_{x y} \text {; } \\
& \sigma_{z^{\prime}}^{\prime}=l_{3}^{2} \sigma_{x}+m_{3}^{2} \sigma_{y}+n_{3}^{2} \sigma_{z}+2 m_{3} n_{3} \tau_{z y}+2 n_{3} l_{3} \tau_{z x}+2 m_{3} l_{3} \tau_{x y} \text {; } \\
& \tau_{y^{\prime} z^{\prime}}^{\prime}=l_{2} l_{3} \sigma_{x}+m_{2} m_{3} \sigma_{y}+n_{2} n_{3} \sigma_{z}+\left(m_{2} n_{3}+m_{3} n_{2}\right) \tau_{y z}+\left(n_{2} l_{3}+n_{3} l_{2}\right) \tau_{z x}+ \\
& +\left(l_{3} m_{2}+l_{2} m_{3}\right) \tau_{x y}
\end{aligned}
$$

В этой системе зависимостей положение новой системы координат $x^{\prime}, y^{\prime}, z^{\prime}$ определяется относительно старой с помощью девяти косинусов между нормалями к площадкам в старой и новой системах, согласно схеме, приведенной в табл. 1.1.

Таблицуа 1.1

Схема к определению направляющих косинусов между двумя прямоугольными системами координат

\begin{tabular}{c|c|c|c}
\hline $\begin{array}{c}\text { Новые } \\
\text { координаты }\end{array}$ & $x$ & $y$ & $z$ \\
\hline$x^{\prime}$ & $l_{1}$ & $m_{1}$ & $n_{1}$ \\
$y^{\prime}$ & $l_{2}$ & $m_{2}$ & $n_{2}$ \\
$z^{\prime}$ & $l_{3}$ & $m_{3}$ & $n_{3}$
\end{tabular}

\section{2. Зависимости деформированного состояния в упругом массиве}

Деформации любого элементарного объема упругого массива могут быть представлены состоящими из ряда отдельных простейших деформаций, т. е. разложены на составляющие. Так, в случае элемен- 
тарного параллелепипеда можно различить шесть составляющих деформаций: три линейные составляющие (относительные удлинения ребер) и три угловые, или сдвиговые, составляющие (относительные изменения первоначальных углов).

Относительные удлинения ребер обозначаются буквой $\varepsilon$ с индексом, указывающим направление удлинения: $\varepsilon_{x} ; \varepsilon_{y} ; \varepsilon_{z}$. Углы сдвига, проектирующиеся на плоскости $x-y, y-z$ и $z-x$, соответственно обозначаются $\gamma_{x y}\left(\right.$ либо $\left.\gamma_{y x}\right), \gamma_{y z}\left(\gamma_{z y}\right)$ и $\gamma_{z x}\left(\gamma_{x z}\right)[1-3]$.

Принято считать, что линейные относительные деформации сопровождаются изменением объема, а угловые относительные деформации - изменением формы выделенного элемента. Однако здесь следует отметить очень важный момент о том, что это условие выполняется не всегда. Например, для упругой объемно недеформируемой среды при различных линейных относительных деформациях, отличных от нуля, изменение объема наблюдаться не будет, так как их сумма равна нулю, а будет наблюдаться формоизменение, не сопровождаемое изменением объема, так называемое чистое. Но об этом речь пойдет дальше.

Если расположить все компоненты, определяющие деформированное состояние в рассматриваемой точке массива, в виде таблицы, подобно таблице напряженного состояния (1.2), то получим матрицу, которую называют тензором деформаций:

$$
T_{\text {деф }}=\left\{\begin{array}{ccc}
\varepsilon_{x}, & \frac{1}{2} \gamma_{x y}, & \frac{1}{2} \gamma_{x z} ; \\
\frac{1}{2} \gamma_{y x}, & \varepsilon_{y}, & \frac{1}{2} \gamma_{y z} ; \\
\frac{1}{2} \gamma_{z x}, & \frac{1}{2} \gamma_{z y}, & \varepsilon_{z} .
\end{array}\right\}
$$

При сложении всех членов по главной диагонали тензора деформаций (1.4) получим относительную объемную деформацию в точке:

$$
\varepsilon_{x}+\varepsilon_{y}+\varepsilon_{z}=\theta \text {. }
$$

Очевидно, что эта сумма инвариантна по отношению к преобразованию ортогональной системы координат, также как и матрица- 
тензор напряженного состояния, поэтому ее называют первым инвариантом тензора деформаций.

Если ввести обозначение

$$
\frac{1}{3}\left(\varepsilon_{x}+\varepsilon_{y}+\varepsilon_{z}\right)=\varepsilon_{\mathrm{cp}}
$$

и назвать его средней деформацией в точке, то очевидно соотношение $\varepsilon_{\mathrm{cp}}=\frac{1}{3} \theta$, в котором $\varepsilon_{\mathrm{cp}}$ представляет первый инвариант деформации в точке.

Подобно тому как это было сделано для напряженного состояния, разложим тензор (1.4) на две составляющие:

$$
T_{\text {деф }}=T_{\text {деф }}^{0}+D_{\text {деф }},
$$

где $T_{\text {деф }}^{0}$ - матрица, именуемая шаровым тензором деформаций:

$$
T_{\partial e \phi}^{0}=\left\{\begin{array}{ccc}
\varepsilon_{\mathrm{cp}}, & 0, & 0 ; \\
0, & \varepsilon_{\mathrm{cp}}, & 0 ; \\
0, & 0, & \varepsilon_{\mathrm{cp}},
\end{array}\right\}
$$

а дополняющей матрицей к (1.7), очевидно, будет матрица

$$
D_{\text {деф }}=\left\{\begin{array}{ccc}
\left(\varepsilon_{x}-\varepsilon_{\mathrm{cp}}\right), & \frac{1}{2} \gamma_{x y}, & \frac{1}{2} \gamma_{x z} ; \\
\frac{1}{2} \gamma_{y x}, & \left(\varepsilon_{y}-\varepsilon_{\mathrm{cp}}\right), & \frac{1}{2} \gamma_{y z} ; \\
\frac{1}{2} \gamma_{z x}, & \frac{1}{2} \gamma_{z y}, & \left(\varepsilon_{z}-\varepsilon_{\mathrm{cp}}\right),
\end{array}\right\}
$$

называемая девиатором деформаций.

Как и сами тензоры напряжений и деформаций, их девиаторы обладают свойствами, раскрывающими характер ожидаемой деформации [1]. 


\section{3. Зависимости компонентов деформаций от компонентов перемещений}

Если в сплошном упругом массиве до его деформации выбрать произвольную точку $M$ с координатами $x, y, z$, которая после деформации займет положение $M_{1}$, то проекции полного перемещения точки в пространстве $M M_{1}$ на оси координат обозначаются соответственно $U, V, W$ и называются компонентами перемещения точки. Очевидно, что эти компоненты в различных точках различны и будут функциями координат точки:

$$
\left.\begin{array}{l}
U=f_{1}(x, y, z) \\
V=f_{2}(x, y, z) \\
W=f_{3}(x, y, z)
\end{array}\right\}
$$

а полное перемещение точки $M$ определяется выражением

$$
\Delta=\sqrt{U^{2}+V^{2}+W^{2}}=\psi(x, y, z) .
$$

Система дифференциальных зависимостей компонентов деформаций от компонентов перемещений, предложенная Коши в 1822 г. [1], записывается в следующем виде:

относительные удлинения

$$
\left.\begin{array}{l}
\varepsilon_{x}=\frac{\partial U}{\partial x} \\
\varepsilon_{\gamma}=\frac{\partial V}{\partial y} \\
\varepsilon_{z}=\frac{\partial W}{\partial z}
\end{array}\right\}
$$

относительные углы сдвига 


$$
\left.\begin{array}{l}
\gamma_{x y}=\frac{\partial U}{\partial y}+\frac{\partial V}{\partial x} \\
\gamma_{y z}=\frac{\partial V}{\partial z}+\frac{\partial W}{\partial y} \\
\gamma_{z x}=\frac{\partial W}{\partial x}+\frac{\partial U}{\partial z} .
\end{array}\right\}
$$

Если около рассматриваемой точки $M(x, y, z)$ выделить элементарный параллелепипед с гранями, параллельными координатным плоскостям, и первоначально предположить, что он не деформируется, то для определения его нового положения в связи с общей деформацией массива трех компонентов перемещений недостаточно, так как он может повернуться вокруг произвольной оси, не параллельной выбранным осям координат. В таком случае нужны составляющие углов поворотов диагоналей сторон параллелепипеда относительно нормальных к ним осей:

$\omega_{\mathrm{x}}-$ угол поворота диагонали грани, параллельной плоскости $y-z$;

$\omega_{\mathrm{y}}-$ угол поворота диагонали грани, параллельной плоскости $z-x$;

$\omega_{z}-$ угол поворота диагонали грани, параллельной плоскости $x-y$, которые называют компонентами жесткого вращения [1]. Они являются функциями координат и тесно связаны с перемещениями.

Эти составляющие компонентов жесткого вращения определяются из зависимостей:

$$
\left.\begin{array}{l}
2 \omega_{x}=\frac{\partial V}{\partial z}-\frac{\partial W}{\partial y} \\
2 \omega_{y}=\frac{\partial W}{\partial x}-\frac{\partial U}{\partial z} \\
2 \omega_{z}=\frac{\partial U}{\partial y}-\frac{\partial V}{\partial x}
\end{array}\right\}
$$

Случай, когда $\omega_{x}=\omega_{y}=\omega_{z}$ (отсутствие жестких поворотов в окресности рассматриваемой точки), в теории упругости называют чистой деформацией, или чистым сдвигом.

Здесь следует отметить, что в теории упругости эти деформации явно недооценены. Например, в [1, с. 87] отмечено, что они «...в об- 
щем для теории упругости интереса не представляют...». Как будет продемонстрировано дальше, они наглядно показывают возможность разделения общей деформации на две независимые части по происхождению: объемную и чистого сдвига.

Дифференциальные зависимости компонентов относительных деформаций (1.8), (1.9) и углов жесткого вращения (1.10) от перемещений называют геометрическими уравнениями.

Уравнения равновесия (1.3) и геометрические зависимости (1.8), (1.9), составляя девять уравнений, содержат 15 неизвестных

$$
\sigma_{x} ; \sigma_{y} ; \sigma_{z} ; \tau_{x y} ; \tau_{y z} ; \tau_{z x} ; \varepsilon_{x} ; \varepsilon_{y} ; \varepsilon_{z} ; \gamma_{x y} ; \gamma_{y z} ; \gamma_{z x} ; U ; V ; W
$$

следовательно, для решения задачи определения напряженно-деформированного состояния упругого массива необходимо добавить еще шесть уравнений. При этом следует уточнить, что система уравнений для жесткого поворота (1.10) не добавляет количества уравнений, так как она вытекает из системы (1.8) и ее можно определить по известным компонентам деформаций.

Перемещения любой точки упругого массива, как уже было отмечено, определяются тремя функциями координат $U ; V ; W$, деформации же элемента вокруг этой точки можно найти с помощью шести функций: $\varepsilon_{x} ; \varepsilon_{y} ; \varepsilon_{z} ; \gamma_{x y} ; \gamma_{y z} ; \gamma_{z x}$.

Из уравнений (1.8) и (1.9) следует, что если заданы три функции перемещений, то компоненты относительных деформаций уже предопределены этими уравнениями.

Очевидно, что все шесть компонентов деформаций задать произвольно нельзя, между ними должны быть какие-то зависимости, которые и были получены Сен-Венаном [1]:

$$
\left.\begin{array}{l}
\frac{\partial^{2} \varepsilon_{x}}{\partial y^{2}}+\frac{\partial^{2} \varepsilon_{y}}{\partial x^{2}}=\frac{\partial^{2} \gamma_{x y}}{\partial x \partial y} ; \quad \frac{\partial}{\partial z}\left(\frac{\partial \gamma_{y z}}{\partial x}+\frac{\partial \gamma_{z x}}{\partial y}-\frac{\partial \gamma_{x y}}{\partial z}\right)=2 \frac{\partial^{2} \varepsilon_{z}}{\partial x \partial y} \\
\frac{\partial^{2} \varepsilon_{y}}{\partial z^{2}}+\frac{\partial^{2} \varepsilon_{z}}{\partial y^{2}}=\frac{\partial^{2} \gamma_{y z}}{\partial y \partial z} ; \quad \frac{\partial}{\partial x}\left(\frac{\partial \gamma_{z x}}{\partial y}+\frac{\partial \gamma_{x y x}}{\partial z}-\frac{\partial \gamma_{y z}}{\partial x}\right)=2 \frac{\partial^{2} \varepsilon_{x}}{\partial y \partial z} \\
\frac{\partial^{2} \varepsilon_{z}}{\partial x^{2}}+\frac{\partial^{2} \varepsilon_{x}}{\partial z^{2}}=\frac{\partial^{2} \gamma_{z x}}{\partial z \partial x} ; \quad \frac{\partial}{\partial y}\left(\frac{\partial \gamma_{x y}}{\partial z}+\frac{\partial \gamma_{y z}}{\partial x}-\frac{\partial \gamma_{z x}}{\partial y}\right)=2 \frac{\partial^{2} \varepsilon_{y}}{\partial z \partial x}
\end{array}\right\}
$$


Система уравнений (1.11) называется условиями совместности, или неразрывности, деформаций. Физический смысл ее следующий: заданный упругий массив, сплошной и непрерывный до деформации, остается таковым и после деформации. Поскольку эта система вытекает из системы геометрических уравнений (1.8), (1.9), то геометрические уравнения также выражают условие неразрывности деформаций и удовлетворение решения задачи системе (1.11) автоматически влечет за собой удовлетворение системе геометрических уравнений.

Как было отмечено выше, все шесть деформаций задать произвольно нельзя, из них произвольно можно задать только три в любой комбинации.

\section{4. Физические зависимости В линейно-упругой изотропной среде}

Итак, для полного описания напряженно-деформированного состояния в упругом массиве необходимо определить 15 неизвестных: шесть компонентов напряжений $\left(\sigma_{x} ; \sigma_{y} ; \sigma_{z} ; \tau_{x y} ; \tau_{y z} ; \tau_{z x}\right)$, шесть компонентов относительных деформаций $\left(\varepsilon_{x} ; \varepsilon_{y} ; \varepsilon_{z} ; \gamma_{x y} ; \gamma_{y z} ; \gamma_{z x}\right)$ и три компонента перемещения $(U ; V ; W)$, для которых имеется шесть уравнений Коши (1.8), (1.9) либо шесть уравнений Сен-Венана (1.11) и три уравнения равновесия (1.3). Недостающие шесть уравнений представляют собой физические зависимости, связывающие напряженное и деформированное состояния. При их обосновании использован принцип независимости действия сил и линейная зависимость деформаций от напряжений.

Полагая вначале действие только напряжений $\sigma_{x}$ (одноосное напряженное состояние), принимается, что элементарный куб получит следующие относительные удлинения [4]:

в направлении оси $x$

$$
\varepsilon_{x}^{\prime}=\frac{\sigma_{x}}{E}
$$

в направлениях осей $y$ и $x$, в соответствии с законом Пуассона: 


$$
\varepsilon_{y}^{\prime}=\varepsilon_{z}^{\prime}=-v \frac{\sigma_{x}}{E}
$$

где $E$ - модуль упругости (постоянный для упругой изотропной среды);

$v$ - коэффициент бокового расширения среды, или коэффициент Пуассона, также постоянный для данной упругой изотропной среды.

Принимая в дальнейшем действие одних только напряжений $\sigma_{y}$, получим:

$$
\varepsilon_{y}^{\prime \prime}=\frac{\sigma_{y}}{E} ; \quad \varepsilon_{x}^{\prime \prime}=\varepsilon_{z}^{\prime \prime}=-v \frac{\sigma_{y}}{E} .
$$

Наконец, рассматривая действие одних только напряжений $\sigma_{z}$, имеем:

$$
\varepsilon_{z}^{\prime \prime \prime}=\frac{\sigma_{z}}{E} ; \quad \varepsilon_{x}^{\prime \prime \prime}=\varepsilon_{y}^{\prime \prime \prime}=-v \frac{\sigma_{z}}{E}
$$

Принято, что касательные напряжения влекут только изменение формы элементарного куба, а удлинения его ребер отсутствуют. При этом пара касательных напряжений $\tau_{x y}$ и $\tau_{y x}$ будет вызывать перекашивание граней элемента, параллельных плоскости хоу, и оставлять без изменения грани $x o z$ и yoz. То же самое относится и к двум остальным парам касательных напряжений $\tau_{y z}=\tau_{z y}$ и $\tau_{z x}=\tau_{x z}$. При линейной зависимости угловой относительной деформации от соответствующего касательного напряжения имеем:

$$
\gamma_{x y}^{\prime}=\frac{\tau_{x y}}{G} ; \quad \gamma_{y z}^{\prime}=\gamma_{z x}^{\prime}=0
$$

где величина $G$, называемая модулем сдвига, реже - постоянной Ламе, как известно [2-4], определяется из условия:

$$
G=\frac{E}{2(1-v)}
$$


Аналогично, при независимом действии других пар касательных напряжений $\tau_{y z}=\tau_{z y}$ и $\tau_{z x}=\tau_{x z}$, будем иметь:

$$
\begin{array}{ll}
\gamma_{y z}^{\prime \prime}=\frac{\tau_{y z}}{G} ; & \gamma_{z x}^{\prime \prime}=\gamma_{x y}^{\prime \prime}=0, \\
\gamma_{z x}^{\prime \prime \prime}=\frac{\tau_{z x}}{G} ; & \gamma_{x y}^{\prime \prime \prime}=\gamma_{y z}^{\prime \prime \prime}=0 .
\end{array}
$$

В итоге, при наличии всех компонентов напряжений, используя принцип независимости действия сил, получим такие обобщенные составляющие деформаций:

$$
\left.\begin{array}{l}
\varepsilon_{x}=\frac{1}{E}\left[\sigma_{x}-v\left(\sigma_{y}+\sigma_{z}\right)\right] ; \\
\varepsilon_{y}=\frac{1}{E}\left[\sigma_{y}-v\left(\sigma_{z}+\sigma_{x}\right)\right] ; \\
\varepsilon_{z}=\frac{1}{E}\left[\sigma_{z}-v\left(\sigma_{x}+\sigma_{y}\right)\right] ; \\
\gamma_{x y}=\frac{\tau_{x y}}{G} ; \quad \gamma_{y z}=\frac{\tau_{y z}}{G} ; \quad \gamma_{z x}=\frac{\tau_{z x}}{G} .
\end{array}\right\}
$$

Первую тройку уравнений из системы (1.12) иногда называют первым законом Гука, а вторую - вторым законом Гука [1].

Из системы (1.12) обратным решением можно получить компоненты напряжений, выраженные через относительные деформации:

$$
\left.\begin{array}{l}
\sigma_{x}=2 G\left(\varepsilon_{x}+\frac{3 \nu}{1-2 \nu} \varepsilon_{\mathrm{cp}}\right) ; \\
\sigma_{y}=2 G\left(\varepsilon_{y}+\frac{3 \nu}{1-2 \nu} \varepsilon_{\mathrm{cp}}\right) ; \\
\sigma_{z}=2 G\left(\varepsilon_{z}+\frac{3 \nu}{1-2 \nu} \varepsilon_{\mathrm{cp}}\right) ; \\
\tau_{x y}=G \gamma_{x y} ; \quad \tau_{y z}=G \gamma_{y z} ; \quad \tau_{z x}=G \gamma_{z x},
\end{array}\right\}
$$

где $\varepsilon_{\mathrm{cp}}$ - средняя линейная деформация в точке, равная (1.6). 
Компоненты нормальных напряжений в (1.13) можно записать также в более компактном виде:

$$
\left.\begin{array}{l}
\sigma_{x}=2 G \varepsilon_{x}+\lambda \theta \\
\sigma_{y}=2 G \varepsilon_{y}+\lambda \theta \\
\sigma_{z}=2 G \varepsilon_{z}+\lambda \theta
\end{array}\right\}
$$

где $\theta$ - объемная относительная деформация в точке, численно равная $\theta=3 \varepsilon_{\mathrm{cp}}$;

$\lambda$ - постоянная Ляме, определяемая зависимостью $[1-3,5]$

$$
\lambda=\frac{2 v}{1-2 v} G \text {. }
$$

После сложения в (1.12) левых и правых частей первых трех уравнений получаем

$$
\varepsilon_{x}+\varepsilon_{y}+\varepsilon_{z}=\frac{1-2 v}{E}\left(\sigma_{x}+\sigma_{y}+\sigma_{z}\right)
$$

Обозначив в (1.15)

$$
\begin{aligned}
& \frac{1}{3}\left(\varepsilon_{x}+\varepsilon_{y}+\varepsilon_{z}\right)=\varepsilon_{\mathrm{cp}}, \\
& \frac{1}{3}\left(\sigma_{x}+\sigma_{y}+\sigma_{z}\right)=\sigma_{\mathrm{cp}}
\end{aligned}
$$

и назвав их соответственно средняя линейная относительная деформация и среднее нормальное напряжение, имеем

$$
\varepsilon_{\mathrm{cp}}=\frac{1-2 v}{E} \sigma_{\mathrm{cp}}
$$

или, обратно

$$
\sigma_{\mathrm{cp}}=\frac{E}{1-2 v} \varepsilon_{\mathrm{cp}}
$$


то есть средняя линейная деформация пропорциональна среднему нормальному напряжению. Учитывая, что сумма относительных удлинений элемента по трем ортогональным направлениям численно равна объемной относительной деформации (1.5), условие (1.16) можно записать выражением

$$
\theta=\frac{3(1-2 v)}{E} \sigma_{\mathrm{cp}},
$$

которое носит название упругого изменения объема [1].

Отняв в первом уравнении системы (1.13) от левой и правой частей соответственно левую и правую часть уравнения (1.14), получим

$$
\sigma_{x}-\sigma_{\mathrm{cp}}=2 G\left(\varepsilon_{x}-\frac{3 \nu}{1-2 \nu} \varepsilon_{\mathrm{cp}}\right)-\frac{E}{1-v} \varepsilon_{\mathrm{cp}},
$$

а окончательно, после замены модуля линейной деформации на модуль сдвига,

$$
\sigma_{x}-\sigma_{\mathrm{cp}}=2 G\left(\varepsilon_{x}-\varepsilon_{\mathrm{cp}}\right)
$$

Второе и третье уравнения системы (1.13) преобразовываются по такой же схеме:

$$
\begin{aligned}
& \sigma_{y}-\sigma_{\mathrm{cp}}=2 G\left(\varepsilon_{y}-\varepsilon_{\mathrm{cp}}\right) ; \\
& \sigma_{z}-\sigma_{\mathrm{cp}}=2 G\left(\varepsilon_{z}-\varepsilon_{\mathrm{cp}}\right) .
\end{aligned}
$$

Остальные три уравнения системы (1.13) остаются без изменений. Полученная таким способом новая система в целом будет иметь вид:

$$
\left.\begin{array}{ll}
\sigma_{x}-\sigma_{\mathrm{cp}}=2 G\left(\varepsilon_{x}-\varepsilon_{\mathrm{cp}}\right) ; & \tau_{x y}=2 G \frac{1}{2} \gamma_{x y} \\
\sigma_{y}-\sigma_{\mathrm{cp}}=2 G\left(\varepsilon_{y}-\varepsilon_{\mathrm{cp}}\right) ; & \tau_{y z}=2 G \frac{1}{2} \gamma_{y z} ; \\
\sigma_{z}-\sigma_{\mathrm{cp}}=2 G\left(\varepsilon_{z}-\varepsilon_{\mathrm{cp}}\right) ; & \tau_{z x}=2 G \frac{1}{2} \gamma_{z x}
\end{array}\right\}
$$


Левые части уравнений в системе (1.18) называют компонентами напряжений, а правые части без коэффициента $2 G$ - компонентами деформаций, отвечающими за изменение формы элемента. В целом система (1.18) представляет закон упругого изменения формы, который формулируется в следующей редакции [1]: компоненты напряжений и деформаций, соответствующие изменению формы, пропорциональны друг другу.

Систему зависимостей (1.18) можно записать также в виде двух таблиц (матриц)

$$
\begin{aligned}
\left\{\begin{array}{ccc}
\sigma_{x}-\sigma_{\mathrm{cp}}, & \tau_{x y}, & \tau_{x z} ; \\
\tau_{y x}, & \sigma_{y}-\sigma_{\mathrm{cp}}, & \tau_{y z} ; \\
\tau_{z x}, & \tau_{z y}, & \sigma_{z}-\sigma_{\mathrm{cp}}
\end{array}\right\} & =\left\{\begin{array}{ccc}
\varepsilon_{x}-\varepsilon_{\mathrm{cp}}, & \frac{1}{2} \gamma_{x y}, & \frac{1}{2} \gamma_{x z} ; \\
\frac{1}{2} \gamma_{y x}, & \varepsilon_{y}-\varepsilon_{\mathrm{cp}}, & \frac{1}{2} \gamma_{y z} ; \\
\frac{1}{2} \gamma_{z x}, & \frac{1}{2} \gamma_{z y}, & \varepsilon_{z}-\varepsilon_{\mathrm{cp}} .
\end{array}\right\}
\end{aligned}
$$

Левую матрицу, составленную из компонентов напряжений, влияющих на изменение формы, называют девиатором напряжений $\left(D_{\text {н }}\right)$, а правую матрицу - соответственно девиатором деформаций, и обобщенный закон упругости будет иметь следующую символическую запись:

$$
D_{\text {н }}=2 G D_{\text {деф }} \text {. }
$$

Выражение (1.20) имеет название закона упругого изменения формы. Возвращаясь к закону изменения объема, выражение (1.17) можно записать так:

$$
T_{\text {н }}^{0}=E_{0} T_{\text {деф }}^{0},
$$

или в матричной форме 


$$
\left\{\begin{array}{ccc}
\sigma_{\mathrm{cp}}, & 0, & 0 \\
0, & \sigma_{\mathrm{cp}}, & 0 ; \\
0, & 0, & \sigma_{\mathrm{cp}},
\end{array}\right\}=E_{0}\left\{\begin{array}{ccc}
\varepsilon_{\mathrm{cp}}, & 0, & 0 \\
0, & \varepsilon_{\mathrm{cp}}, & 0 ; \\
0, & 0, & \varepsilon_{\mathrm{cp}}
\end{array}\right\}
$$

В (1.21) и (1.22) коэффициент пропорциональности $E_{0}$ называют объемным модулем упругости, который имеет следующую зависимость от линейного модуля упругости:

$$
E_{0}=\frac{E}{3(1-2 v)} \text {. }
$$

\section{5. Плоское деформирование упругой среды}

Если при каком-либо напряженном состоянии упругого массива перемещения всех его точек будут происходить только в одной плоскости, то такую деформацию называют плоской. Классическим примером плоской деформации может быть упругое полупространство, на поверхности которого равномерно распределена нагрузка вдоль бесконечной линии или полосы постоянной ширины. Сюда также относятся упругие массивы, имеющие ограниченную толщину и хотя бы один из оставшихся размеров бесконечной величины, вдоль которого равномерно распределена внешняя нагрузка, а именно:

бесконечно простирающийся массив ограниченной постоянной толщины на жестком основании;

массив бесконечной длины и ограниченных, но постоянных по длине поперечных размеров на жестком основании.

Примером плоской деформации упругого тела малых размеров может быть тело цилиндрической формы, размещенное без трения между двух параллельных абсолютно жестких пластин с фиксированным расстоянием между ними и нагруженное на боковой поверхности равномерной нагрузкой $p$ (рис. 1.3).

Если упругий массив с фиксированными поперечными размерами имеет бесконечно большую длину вдоль оси $y$ и в этом же направлении равномерно распределена внешняя нагрузка, то перемещения всех точек деформированного тела будут происходить только в плос- 
костях, перпендикулярных линии распределения внешней нагрузки (оси y), т. е. $\varepsilon_{y}=0 ; \quad \gamma_{x y}=0 ; \quad \gamma_{z y}=0 ; \quad \tau_{x y}=0 ; \quad \tau_{y z}=0$, а тензор напряженного состояния в этом случае будет иметь следующую запись:

$$
T_{\mathrm{H}}=\left\{\begin{array}{ccc}
\sigma_{x} ; & 0 ; & \tau_{x z} ; \\
0 ; & \sigma_{y} ; & 0 ; \\
\tau_{z x} ; & 0 ; & \sigma_{z} .
\end{array}\right\}
$$

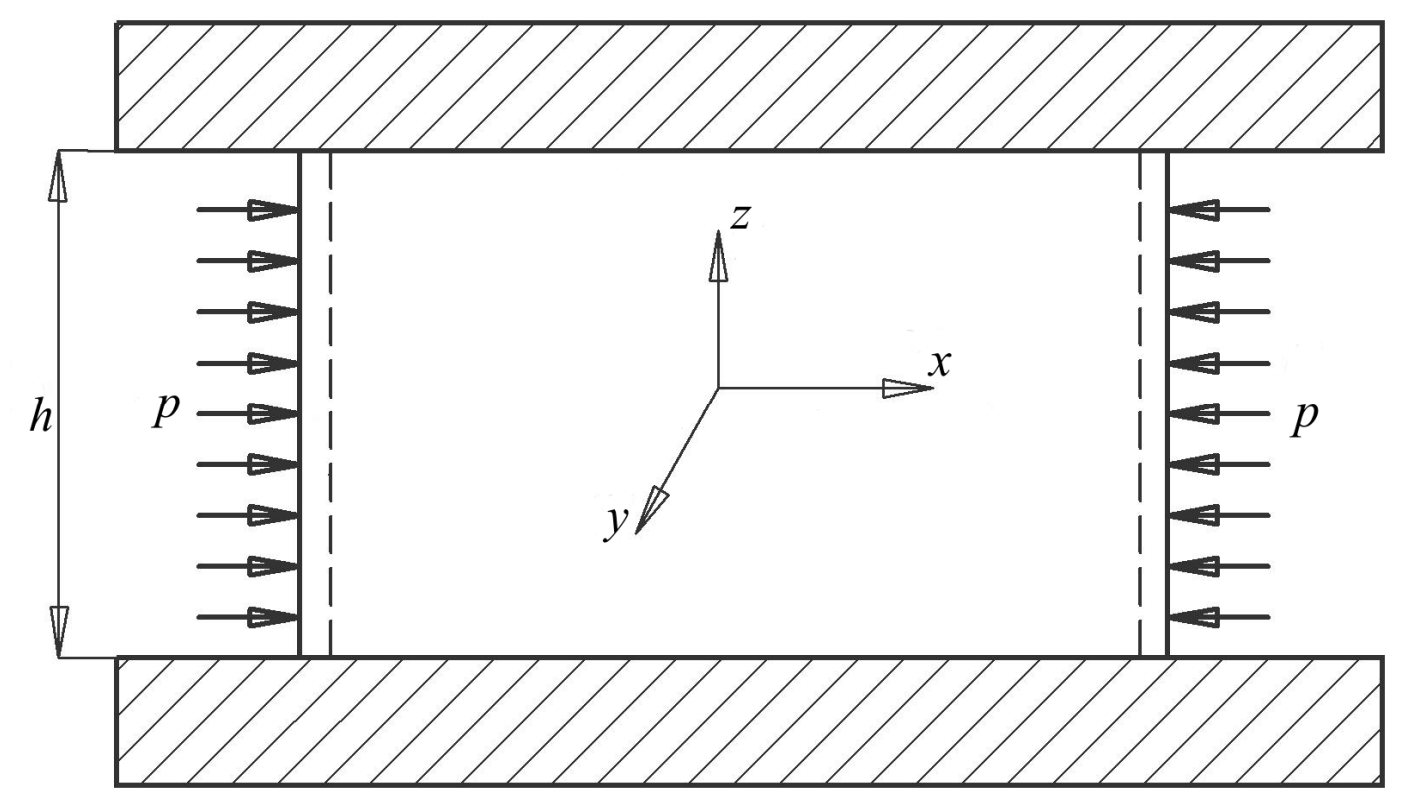

Рис. 1.3. Упругое тело между двух жестких пластин, нагруженное на боковой поверхности равномерной нагрузкой (плоская деформация)

Система уравнений равновесия (1.3) для плоской деформации упрощается и принимает вид

$$
\left.\begin{array}{c}
\frac{\partial \sigma_{x}}{\partial x}+\frac{\partial \tau_{z x}}{\partial z}=0 \\
\frac{\partial \tau_{x z}}{\partial x}+\frac{\partial \sigma_{z}}{\partial z}=0
\end{array}\right\}
$$

Из обобщенного физического закона, полагая $\varepsilon_{y}=0$, получим:

$$
\sigma_{y}=v\left(\sigma_{x}+\sigma_{z}\right)
$$


и тогда относительные деформации будут определяться зависимостями

$$
\left.\begin{array}{l}
\varepsilon_{x}=\frac{1}{E}\left[\left(1-v^{2}\right) \sigma_{x}-v(1+v) \sigma_{z}\right] \\
\varepsilon_{z}=\frac{1}{E}\left[\left(1-v^{2}\right) \sigma_{z}-v(1+v) \sigma_{x}\right] .
\end{array}\right\}
$$

Из уравнений непрерывности деформаций (1.10) остается одно:

$$
\frac{\partial^{2} \varepsilon_{x}}{\partial z^{2}}+\frac{\partial^{2} \varepsilon_{z}}{\partial x^{2}}=\frac{\partial^{2} \gamma_{x z}}{\partial x \partial z}
$$

Если деформации в (1.25) выразить через напряжения, то получим:

$$
\frac{\partial^{2}}{\partial z^{2}}\left(\sigma_{x}-v \sigma_{z}\right)+\frac{\partial^{2}}{\partial x^{2}}\left(\sigma_{z}-v \sigma_{x}\right)=2(1+v) \frac{\partial^{2} \tau_{x z}}{\partial x \partial z}
$$

то есть это уравнение представляет условие совместности деформаций, выраженное в напряжениях.

Продифференцировав первое уравнение в (1.23) по $x$, а второе по $z$ и затем сложив их, получим:

$$
2 \frac{\partial^{2} \tau_{x z}}{\partial x \partial z}=-\frac{\partial^{2} \sigma_{x}}{\partial x^{2}}-\frac{\partial^{2} \sigma_{z}}{\partial z^{2}}
$$

Подстановка (1.24) в уравнение (1.25) приводит уравнение совместности к виду, выраженному только через нормальные напряжения, т. е. получаем известное уравнение Леви [1]:

$$
\frac{\partial^{2}}{\partial x^{2}}\left(\sigma_{x}+\sigma_{z}\right)+\frac{\partial^{2}}{\partial z^{2}}\left(\sigma_{x}+\sigma_{z}\right)=0
$$

В итоге, для плоской деформации совокупность основных уравнений теории упругости приводится к системе из трех уравнений: 


$$
\begin{gathered}
\frac{\partial \sigma_{x}}{\partial x}+\frac{\partial \tau_{x z}}{\partial z}=0 \\
\frac{\partial \tau_{z x}}{\partial x}+\frac{\partial \sigma_{z}}{\partial z}=0 \\
\nabla^{2}\left(\sigma_{x}+\sigma_{z}\right)=0
\end{gathered}
$$

где для сокращения записи принято:

$$
\nabla^{2}=\left(\frac{\partial^{2}}{\partial x^{2}}+\frac{\partial^{2}}{\partial z^{2}}\right)
$$

то есть эта запись представляет собой известный оператор Лапласа.

Таким образом, сумма нормальных напряжений $\left(\sigma_{x}+\sigma_{z}\right)$ в задаче плоского деформирования, инвариантная относительно выбора координатной системы, представляет гармоническую, а следовательно потенциальную функцию. 
ГЛАВА 2

\section{Анализ напряженно-деформированного состояния упругих массивов \\ в решениях теории упругости}

Далее в данной работе рассмотрим и проанализируем решения теории упругости, которые считаются математически точными, т. е. удовлетворяющими всем ее требованиям, и поэтому стали классическими. К таким решениям относят решения Буссинеска [1-6], Фламана [2; 7-9], Кельвина $[4 ; 10 ; 11]$.

\section{1. Решение Буссинеска}

На поверхности полупространства по нормали к нему действует сосредоточенная сила $P$. Начало координат принято в точке приложения силы, ось $z$ направлена вниз, в полупространство. Эта задача решена французским ученым Буссинеском в 1885 г. [6]. Запишем это решение в декартовой системе координат.

Компоненты напряжений

$$
\begin{gathered}
\sigma_{x}=\frac{3 P}{2 \pi}\left\{\frac{x^{2} z}{R^{5}}+\frac{(1-2 v)}{3}\left[\frac{1}{R(R+z)}-\frac{(2 R+z) x^{2}}{R^{3}(R+z)^{2}}-\frac{z}{R^{3}}\right]\right\} ; \\
\sigma_{y}=\frac{3 P}{2 \pi}\left\{\frac{y^{2} z}{R^{5}}+\frac{(1-2 v)}{3}\left[\frac{1}{R(R+z)}-\frac{(2 R+z) y^{2}}{R^{3}(R+z)^{2}}-\frac{z}{R^{3}}\right]\right\} ; \\
\sigma_{z}=\frac{3 P}{2 \pi} \frac{z^{3}}{R^{5}} ;
\end{gathered}
$$




$$
\begin{gathered}
\tau_{x y}=\frac{3 P}{2 \pi}\left[\frac{x y z}{R^{5}}-\frac{(1-2 v)}{3} \frac{(2 R+z) x y}{R^{3}(R+z)^{2}}\right] \\
\tau_{y z}=\frac{3 P}{2 \pi} \frac{y z^{2}}{R^{5}} \\
\tau_{z x}=\frac{3 P}{2 \pi} \frac{x z^{2}}{R^{5}}
\end{gathered}
$$

первый инвариант напряженного состояния

$$
I_{1}=\sigma_{x}+\sigma_{y}+\sigma_{z}=\frac{3 P}{2 \pi} \frac{z}{R^{3}}-\frac{(1-2 v)}{3} \frac{3 P}{2 \pi} \frac{z}{R^{3}}=(1+v) \frac{P z}{\pi R^{3}} ;
$$

компоненты перемещений

$$
\begin{gathered}
U=\frac{P(1+v)}{2 \pi E}\left[\frac{x z}{R^{3}}-(1-2 v) \frac{x}{R(R+z)}\right] ; \\
V=\frac{P(1+v)}{2 \pi E}\left[\frac{y z}{R^{3}}-(1-2 v) \frac{y}{R(R+z)}\right] ; \\
W=\frac{P(1+v)}{2 \pi E}\left[\frac{z^{2}}{R^{3}}-2(1-v) \frac{1}{R}\right] .
\end{gathered}
$$

Решение этой задачи в цилиндрических координатах записывается в следующем виде [1]:

$$
\begin{gathered}
\sigma_{z}=\frac{3 P}{2 \pi} \frac{z^{3}}{R^{5}} \\
\sigma_{r}=-\frac{P}{2 \pi}\left[\frac{(1-2 v)}{R(R+z)}-\frac{3 r^{2} z}{R^{5}}\right]
\end{gathered}
$$




$$
\begin{gathered}
\sigma_{\theta}=-\frac{P}{2 \pi}(1-2 v)\left[\frac{z}{R^{3}}-\frac{1}{R(R+z)}\right] \\
\tau_{r z}=\frac{3 P}{2 \pi} \frac{r z^{2}}{R^{5}}
\end{gathered}
$$

компонент горизонтальных перемещений

$$
U_{r}=\frac{P(1+v)}{2 \pi E}\left[\frac{z r}{R^{3}}-(1-2 v) \frac{r}{\left(R^{2}+r z\right)}\right]
$$

компонент вертикальных перемещений

$$
W=\frac{P(1+v)}{2 \pi E}\left[\frac{2(1+v)}{R}+\frac{z^{2}}{R^{3}}\right] .
$$

В формулах (2.1)-(2.16):

$R$ - радиус-вектор, расстояние от рассматриваемой точки с координатами $x, y, z$ до точки приложения внешней силы,

$$
R=\left(x^{2}+y^{2}+z^{2}\right)^{1 / 2}
$$

$v$ - коэффициент Пуассона;

$E$ - модуль линейной деформации;

$r$ - горизонтальное расстояние от рассматриваемой точки до оси $z$ (цилиндрическая координата),

$$
r=\left(x^{2}+y^{2}\right)^{1 / 2}
$$

Здесь и в дальнейших выкладках значение сжимающего напряжения принято со знаком плюс.

В представленном решении имеются следующие недостатки. Компоненты нормальных напряжений $\sigma_{x}$ и $\sigma_{y}(2.1)$ и (2.2) в декартовой системе координат либо $\sigma_{r}$ и $\sigma_{\theta}(2.12)$ и (2.13) в цилиндрической системе координат зависят от деформационной характеристики $v$, 
и эта зависимость приводит к необоснованному появлению по оси $x$ и $y$ поверхности полупространства (при $z=0$ ) растягивающих горизонтальных напряжений при сжимающей внешней силе $P$ и, наоборот, сжимающих горизонтальных напряжений при растягивающей внешней силе:

$$
\begin{aligned}
& r=0 ; \quad z \neq 0 \rightarrow \sigma_{r}=-\frac{P}{2 \pi} \frac{(1-2 v)}{2 z^{2}} ; \\
& r \neq 0 ; \quad z=0 \rightarrow \sigma_{r}=-\frac{P}{2 \pi} \frac{(1-2 v)}{z^{2} r^{2}} .
\end{aligned}
$$

Появление радиального напряжения $\sigma_{r}$ в упругом массиве - это реакция его на действие внешней силы $P$, поэтому невозможно представить, чтобы при сжатии любого тела внешней вертикальной силой в нем возникали растягивающие горизонтальные напряжения и, наоборот, при растяжении внешней силой возникали сжимающие горизонтальные напряжения. Это противоречит кинематике деформирования.

Полное напряженное состояние в решении (2.1)-(2.7) можно разделить на две части: в одной компоненты напряжений не зависят от коэффициента Пуассона (v), а в другой - зависят. Запишем эти части в виде двух систем.

Система напряжений, не зависящих от v:

компоненты напряженного состояния

$$
\left.\begin{array}{ll}
\sigma_{x}^{\prime}=\frac{3 P}{2 \pi} \frac{x^{2} z}{R^{5}} ; & \tau_{x y}^{\prime}=\frac{3 P}{2 \pi} \frac{x y z}{R^{5}} ; \\
\sigma_{y}^{\prime}=\frac{3 P}{2 \pi} \frac{y^{2} z}{R^{5}} ; & \tau_{y z}^{\prime}=\frac{3 P}{2 \pi} \frac{y z^{2}}{R^{5}} ; \\
\sigma_{z}^{\prime}=\frac{3 P}{2 \pi} \frac{z^{3}}{R^{5}} ; \quad \tau_{z x}^{\prime}=\frac{3 P}{2 \pi} \frac{x z^{2}}{R^{5}} ;
\end{array}\right\}
$$

первый инвариант напряженного состояния:

$$
I_{1}^{\prime}=\sigma_{x}^{\prime}+\sigma_{y}^{\prime}+\sigma_{z}^{\prime}=\frac{3 P}{2 \pi} \frac{z}{R^{3}} .
$$


Система напряжений, зависимых от v: компоненты напряженного состояния

$$
\begin{aligned}
& \sigma_{x}^{\prime \prime}=\frac{3 P}{2 \pi} \frac{(1-2 v)}{3}\left[\frac{1}{R(R+z)}-\frac{x^{2}(2 R+z)}{R^{3}(R+z)^{2}}-\frac{z}{R^{3}}\right] ; \\
& \sigma_{y}^{\prime \prime}=\frac{3 P}{2 \pi} \frac{(1-2 v)}{3}\left[\frac{1}{R(R+z)}-\frac{y^{2}(2 R+z)}{R^{3}(R+z)^{2}}-\frac{z}{R^{3}}\right] ; \\
& \sigma_{z}^{\prime \prime}=0 ; \\
& \tau_{x y}^{\prime \prime}=-\frac{3 P}{2 \pi} \frac{(1-2 v)}{3} \frac{x y(2 R+z)}{R^{3}(R+z)^{2}} \\
& \tau_{y z}^{\prime \prime}=0 \\
& \tau_{z x}^{\prime \prime}=0
\end{aligned}
$$

первый инвариант напряженного состояния

$$
I_{1}^{\prime \prime}=\sigma_{x}^{\prime \prime}+\sigma_{y}^{\prime \prime}+\sigma_{z}^{\prime \prime}=-\frac{(1-2 v)}{3} \frac{3 P}{2 \pi} \frac{z}{R^{3}} .
$$

Нетрудно убедиться в том, что обе системы напряжений (2.17), (2.19) удовлетворяют дифференциальным условиям равновесия (1.3).

При сравнении инвариантов (2.18) и (2.20) стает очевидным, что они описываются одной и той же гармонической функцией, отвечающей одним и тем же граничным условиям. Однако если инвариант (2.18) вызван действием приложенной силы $P$, то инвариант (2.20) соответствует действию силы $-\frac{1-2 v}{3} P$.

Таким образом, полный инвариант напряженного состояния (2.7) вызван действием на полупространство двух приложенных в одной точке, различных по величине и противоположно направленных сил Р и $-\frac{1-2 v}{3} P$.

Однако вторая сила является фиктивной: в равновесии она участия не принимает, но ее действие уменьшает общую интенсивность напряженного состояния и, в частности, величину компонентов $\sigma_{x}$ 
и $\sigma_{y}$. Как это объяснить с точки зрения физики, механики, математики, логики? Вопрос пока остается открытым.

Из решения следует, что горизонтальные смещения поверхности, ограничивающей полупространство, направлены к точке приложения сжимающей внешней силы $P$ и от нее, когда она растягивающая.

Так, приняв в (2.15) $z=0$, имеем:

$$
U_{r}=-\frac{(1-2 v)(1+v) P}{2 \pi E r} .
$$

На рис. 2.1 показана качественная эпюра горизонтальных смещений поверхности от силы $P$, на которой очевиден разрыв сплошности среды в точке приложения силы. Объяснить этот разрыв лишь особенностью распределения напряженно-деформированного состояния в этой точке и решить проблему исключением из рассмотрения некоторой ограниченной области вокруг нее неправомерно, так как при экстраполировании данного решения на распределенную каким-либо образом нагрузку на поверхности полупространства этот разрыв сохраняется. Проиллюстрируем это следующим примером.

Вертикальные перемещения поверхности полупространства от сосредоточенной силы согласно (2.16) при $z=0$ будут

$$
W=\frac{\left(1-v^{2}\right) P}{\pi E r} .
$$

Интегрирование (2.22) для случая равномерно распределенной нагрузки интенсивностью $q$ по площади круга радиуса $a$ (согласно рис. 2.2) приводит к следующему результату [4]:

вертикальные перемещения центра загруженной площади

$$
W_{r=0}=\frac{2\left(1-v^{2}\right)}{E} q a ;
$$

вертикальные перемещения края загруженной площади

$$
W_{r=a}=\frac{4\left(1-v^{2}\right)}{\pi E} q a .
$$




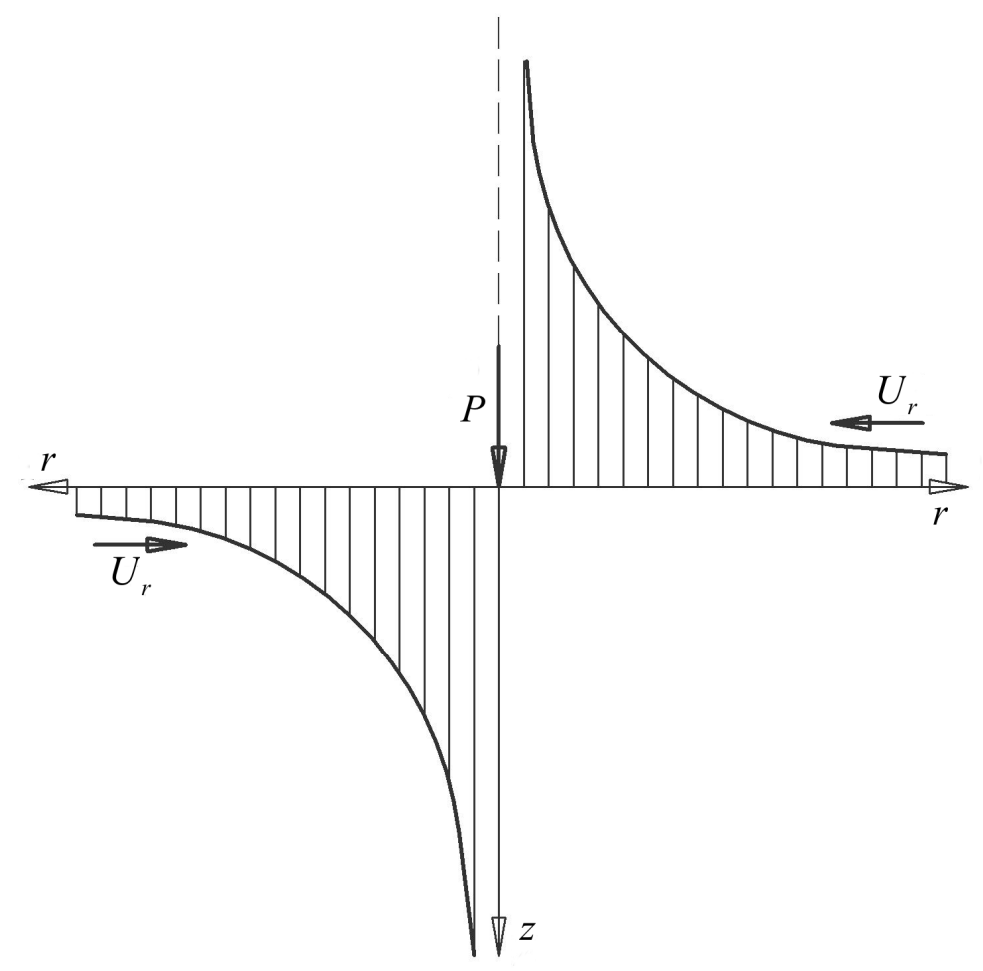

Рис. 2.1. Качественная эпюра горизонтальных смещений поверхности полупространства от силы $P$ в решении Буссинеска

Поскольку исходные функции для определения горизонтальных и вертикальных смещений, соответственно (2.21) и (2.22), отличаются лишь постоянным коэффициентом, равным

$$
k=\frac{U_{r=0}}{W_{r=0}}=-\frac{(1-2 v)(1+v) P}{2 \pi E r} \frac{\pi E r}{(1-v)(1+v) P}=-\frac{1-2 v}{2(1-v)},
$$

то горизонтальные перемещения поверхности в тех же точках, выраженные через вертикальные, будут:

$$
\begin{gathered}
U_{r=0}=k W_{r=0}=-\frac{(1-2 v)(1+v)}{2(1-v)} \frac{2(1-v)}{E} q a=-\frac{(1-2 v)(1+v)}{E} q a \\
U_{r=a}=k W_{r=a}=-\frac{(1-2 v)}{2(1-v)} \frac{4\left(1-v^{2}\right)}{\pi E} q a=-\frac{2(1-2 v)(1+v)}{\pi E} q a
\end{gathered}
$$


то есть горизонтальное смещение центра загруженной площади в $\pi / 2$ раза больше смещения ее края. Соответствующая эпюра качественно приведена на рис. 2.2.

Таким образом, разрыв горизонтальных перемещений поверхности в исходной формуле (2.21) для сосредоточенной силы сохраняется при экстраполяции ее на распределенную нагрузку и приводит к нелепому результату.

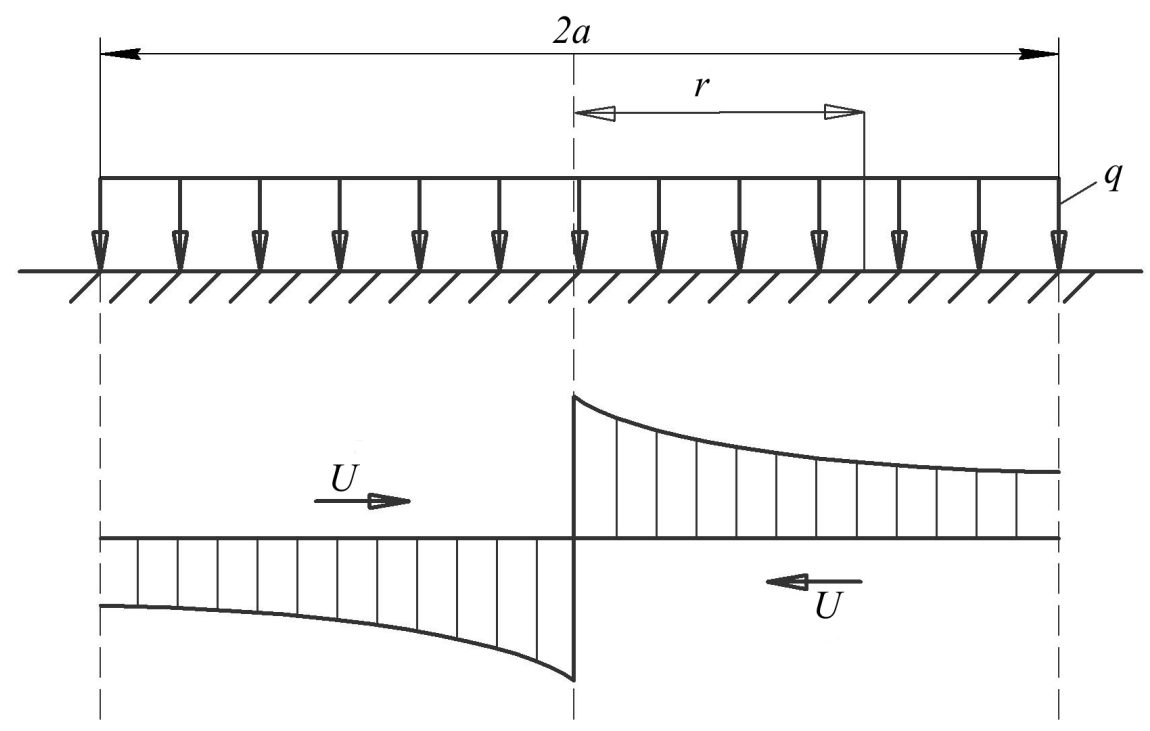

Рис. 2.2. Качественная эпюра горизонтальных перемещений

поверхности над нагрузкой, равномерно распределенной по площади круга

Наличие горизонтальных смещений точек плоскости, ограничивающей упругое полупространство, приводит к тому, что ее деформированное положение при симметричном изменении направления действия силы $P$ на противоположное изменится несимметрично. Так, если на поверхности полупространства выбрать какую-либо точку $M$ (рис. 2.3), то после приложения вертикальной сжимающей силы $(+P)$ точка опустится вниз и приблизится к направлению действия силы, заняв положение $M_{1}$ (рис. $2.3, a$ ). После симметричного изменения направления действия внешней силы на растягивающее $(-P)$ выбранная точка $M$ поднимется вверх и удалится от точки приложения силы, заняв положение $M_{2}$ (рис. $\left.2.3, \sigma\right)$.

При нагрузке упругого полупространства нормально направленной к его поверхности сосредоточенной силой $P$ в нем возникают 
радиальные горизонтальные перемещения (2.15), которые можно представить в виде [5]

$$
U_{r}=\frac{P \sin \theta}{4 \pi G r}\left(\cos \theta-\frac{1-2 v}{1+\cos \theta}\right),
$$

где $\theta$ - угол, образованный радиус-вектором с вертикальной осью $z$, направленной вниз (рис. 2.4);

$G$ - модуль сдвига;

$r$ - горизонтальное расстояние от рассматриваемой точки до оси $z$ (радиус),

$$
r=\sqrt{x^{2}+y^{2}} .
$$

$a$

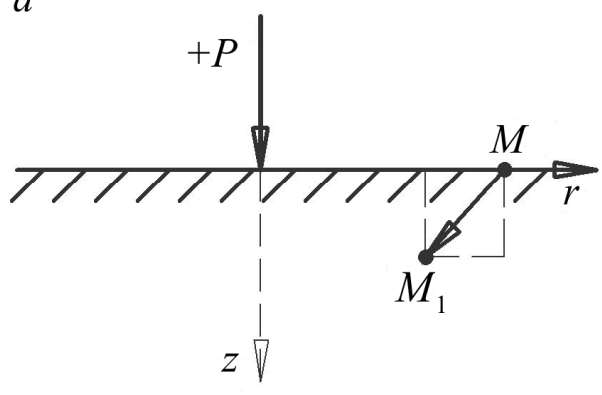

$\sigma$

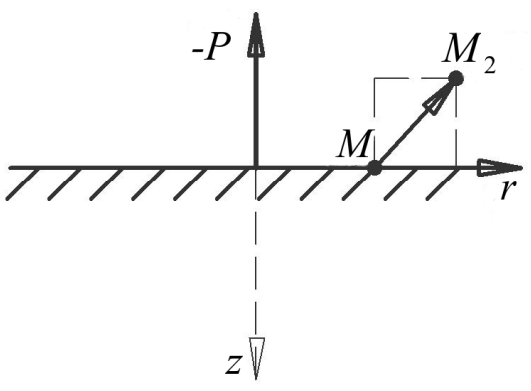

Рис. 2.3. Смещение точки поверхности полупространства от сосредоточенной силы в решении Буссинеска:

$a$ - сила, сжимающая полупространство; $\sigma$ - сила, растягивающая полупространство

Смещение направлено либо к линии действия силы $P$, либо в противоположную сторону, в зависимости от того, будет ли радиусвектор $R$ лежать вне или внутри конической области, которая описывается уравнением

$$
\cos \theta(1+\cos \theta)=(1-2 v) .
$$

Предельные значения угла $\theta$, в зависимости от коэффициента Пуассона, будут:

$$
\begin{array}{ll}
v=0, & \theta_{\text {min }}=51^{\circ} 40^{\prime} ; \\
v=0,5, & \theta_{\text {max }}=0,5 \pi .
\end{array}
$$


Пользуясь формулой (2.23), можно построить эпюру горизонтальных перемещений $U_{r}$ на некоторой глубине $z$ в решении Буссинеска. Такая эпюра качественно показана на рис. 2.4, где угол $\theta$ удовлетворяет условию (2.25). Анализ этой эпюры приводит к следующим результатам.

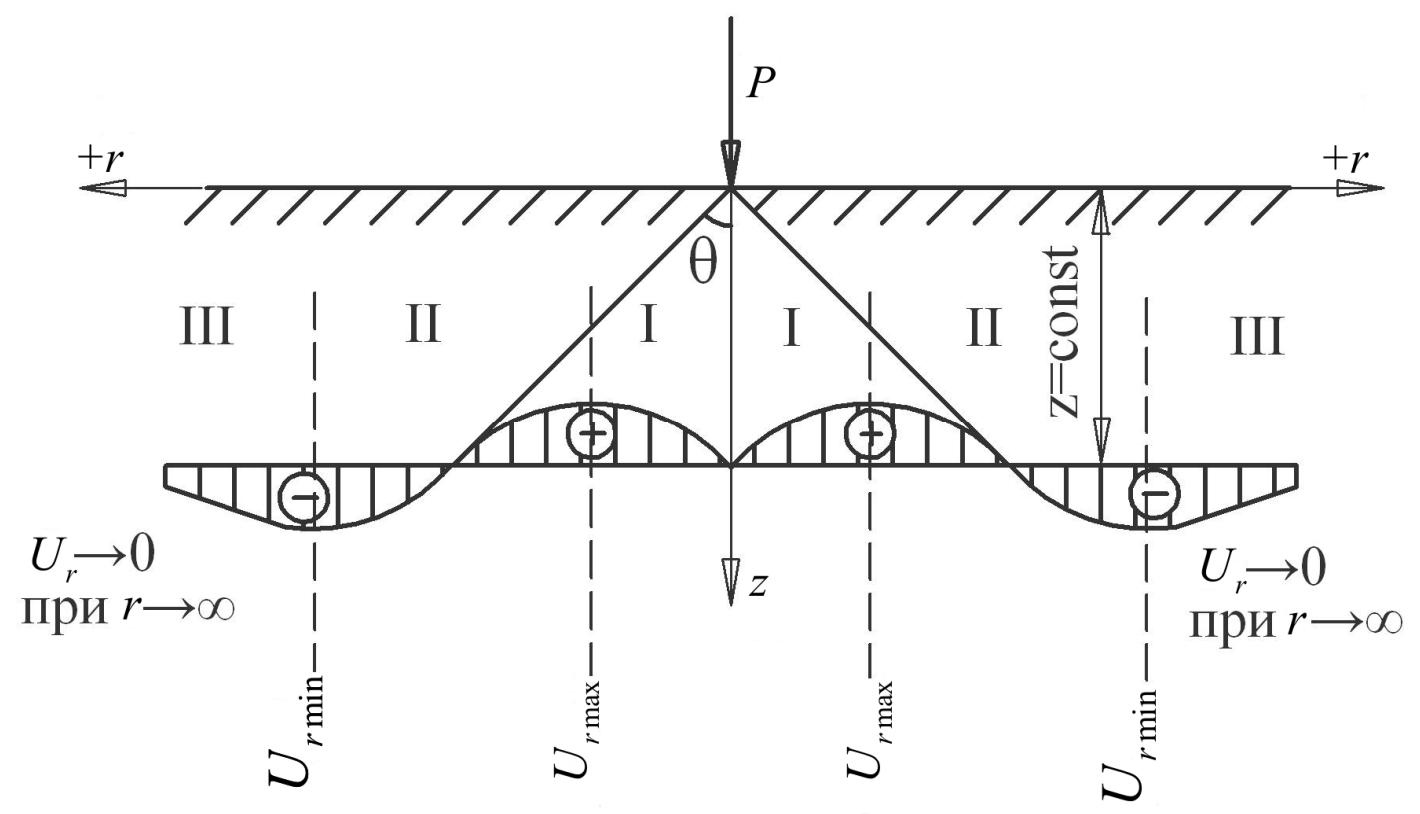

Рис. 2.4. Качественная эпюра горизонтальных смещений точек полупространства от сосредоточенной силы в плоскости $z=$ const (решение Буссинеска)

Начиная от оси $z$, горизонтальные перемещения положительны, то есть совпадают с направлением радиуса $r$, и увеличиваются от нуля до своего максимального значения $U_{r \max }$, а затем уменьшаются до нуля в точке пересечения образующей конической области с горизонталью. В этой точке перемещения меняют свое направление на противоположное, т. е. становятся отрицательными. Продолжая уменьшаться, они достигают своего минимального значения $U_{r \min }$, после чего начинают возрастать и постепенно, при $r \rightarrow \infty$, приближаются к нулю.

Если рассматривать деформации в направлении радиуса $r$, то на нем можно выделить три области. Первая начинается от оси $z$ и заканчивается в точке достижения горизонтальными перемещениями максимального значения $U_{r \max }$. В этой области упругая среда 
испытывает растяжение по горизонтали $\left(\varepsilon_{r}>0\right)$. Вторая область начинается в точке $U_{r \max }$ и заканчивается в точке $U_{r \min }$. Здесь относительные горизонтальные деформации меняют свой знак на отрицательный $\left(\varepsilon_{r}<0\right)$ и среда по горизонтали начинает сжиматься. Третья область начинается в точке достижения горизонтальными перемещениями своего минимального значения $U_{r \min }$ и продолжается до $r \rightarrow \infty$. На этом участке относительные горизонтальные деформации меняют свой знак и становятся растягивающими $\left(\varepsilon_{r}>0\right)$.

Таким образом, горизонтальные перемещения точек среды вдоль радиуса $r$ меняют свое направление, а относительная линейная деформация $\varepsilon_{r}$ меняет знак дважды: растяжение-сжатие-растяжение, что не может быть оправдано кинематикой деформирования. Для качественного уточнения возможных горизонтальных перемещений проведем следующий мысленный эксперимент.

Приложим вертикальную силу $P$ в центре верха упругого тела цилиндрической формы диаметром $2 a$ и высотой $h$, опирающегося без трения на недеформируемое основание (рис. 2.5).

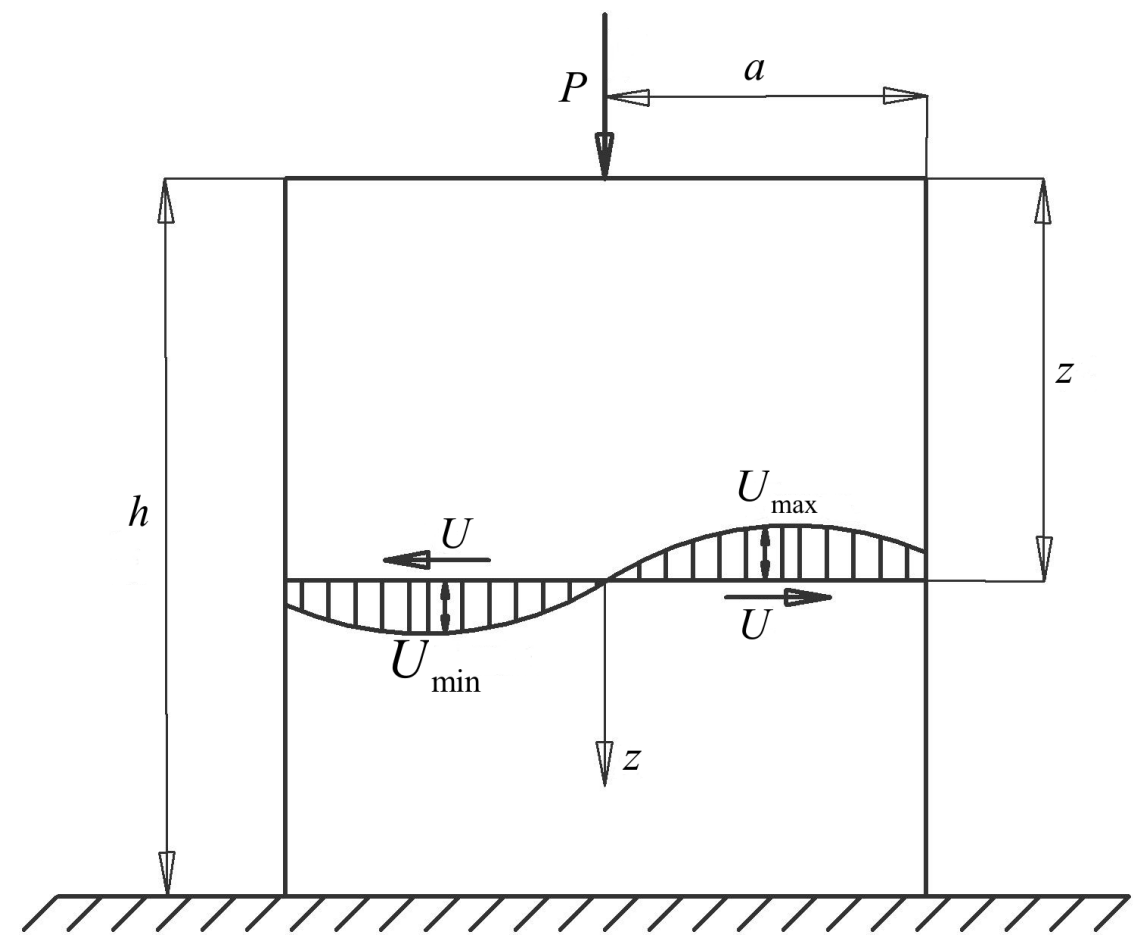

Рис. 2.5. Качественная эпюра кинематически возможных горизонтальных перемещений в упругом теле цилиндрической формы 
Очевидно, что в этом случае тело будет расширяться в горизонтальных направлениях от оси симметрии $z$, т. е. в нем возникнут соответствующие перемещения, качественная эпюра для которых показана на упомянутом выше рисунке. Если теперь мы будем неограниченно увеличивать высоту и радиус цилиндра при неизменной силе $P$, то горизонтальные перемещения боковой поверхности тела будут монотонно уменьшаться и в пределе $(a=\infty), \quad(h=\infty)$ станут равными нулю.

Максимальное значение горизонтального смещения также будет уменьшаться и стремиться к какому-то конечному положительному значению, однако для изменения направления смещений внутри тела нет никаких причин, это кинематически невозможно. Поэтому причину изменения направления горизонтальных перемещений в решении Буссинеска следует искать в исходных предпосылках, т. е. в физических зависимостях, которые не точно отражают взаимосвязь между напряженным и деформированным состояниями среды. Устранить эту аномалию можно путем уточнения физических зависимостей.

Продолжая анализ решения Буссинеска, выделим в упругом полупространстве часть его объема вместе с приложенной внешней силой $P$ некоторой замкнутой на ограничивающую плоскость поверхностью (рис. 2.6), а действие отброшенной части заменим множеством сил $q_{i}$, сосредоточенных на малых площадках.

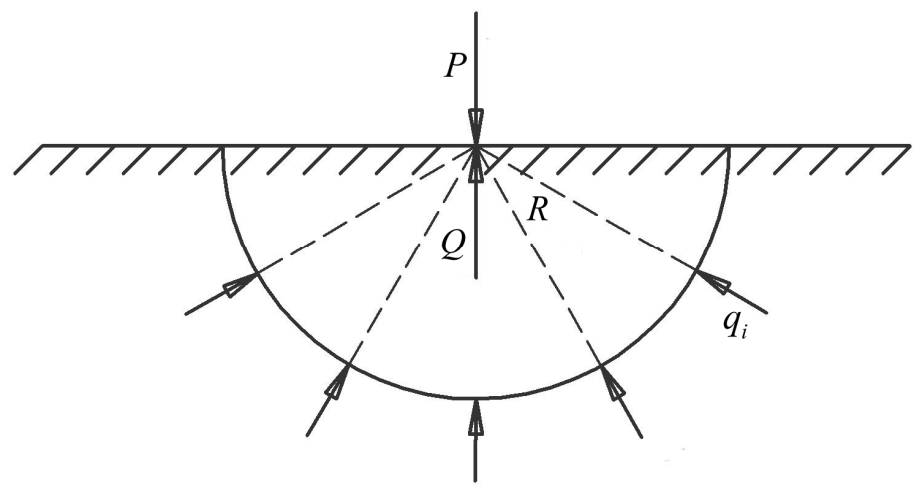

Рис. 2.6. Радиальность напряженного состояния в полупространстве

На рис. 2.6 форма выделенного объема изображена в виде полусферы, но она может быть любой, что никак не меняет сути рассматриваемого вопроса. 
Согласно третьему закону механики реактивная сила $Q$ должна быть равной внешней силе $P$, противоположно ей направленной и приложенной в той же точке, что и сила $P$. Действие же сил $q_{i}$ должно быть статически эквивалентным силе $Q$, поэтому она должна быть их равнодействующей, но равнодействующую имеет только та система сил, линии действия которых пересекаются в одной точке. Такой точкой является точка приложения внешней силы. Отсюда вывод: всякая элементарная реактивная сила $q_{i}$ внутри полупространства, сосредоточенная на любой элементарной площадке, должна быть направлена по радиус-вектору $R$ в точку приложения внешней силы на его поверхности.

Этим самым полностью определяется напряженное состояние в полупространстве, так как элементарная сила $q_{i}$, отнесенная к величине площадки действия $\Delta F_{i}$, представляет полное напряжение в этой площадке, которое называется радиальным: $\sigma_{R}=\frac{q_{i}}{\Delta F_{i}}$.

Очевидно, когда элементарная площадка ориентирована нормально к радиус-вектору в некоторой точке, то такое напряжение будет главным, а два другие будут отсутствовать. Именно такое напряженное состояние в полупространстве вытекает из третьего закона механики.

А теперь проверим, соблюдается ли этот закон в решении Буссинеска. С этой целью выделим элементарную площадку в полупространстве, нормалью к которой является радиус $r$ - расстояние до линии действия силы $P$ (рис. 2.7 в цилиндрической системе координат).

Действующее полное напряжение $\sigma_{R}$ в этой площадке разложим на два компонента:

$$
\sigma_{r}=\sigma_{R} \sin \alpha=\sigma_{R} \frac{r}{R} ; \quad \tau_{r z}=\sigma_{R} \cos \alpha=\sigma_{R} \frac{z}{R} .
$$

Соотношение

$$
\frac{\sigma_{r}}{\tau_{r z}}=\frac{\sigma_{R} \sin \alpha}{\sigma_{R} \cos \alpha}=\operatorname{tg} \alpha=\frac{r}{z}
$$

является условием радиальности напряженного состояния. 
Подставим в (2.26) значения нормального $\sigma_{r}$ (2.12) и тангенциального $\tau_{r z}(2.14)$ напряжений:

$$
\frac{\sigma_{r}}{\tau_{r z}}=\frac{P}{2 \pi}\left[\frac{3 z r^{2}}{R^{5}}-\frac{(1-2 v)}{R(R+z)}\right] \frac{2 \pi R^{5}}{3 P z^{2} r} .
$$

Очевидно, что условие радиальности напряженного состояния (2.28), так же как и третий закон механики, в общем случае в решении Буссинеска не выполняются. Однако есть частный случай, единственный из множества случаев напряженного состояния с различными коэффициентами Пуассона, в котором при $v=0,5$ выполняются и условия равновесия, и третий закон механики, - это радиальное напряженное состояние, в котором нет места его зависимости от коэффициента Пуассона.

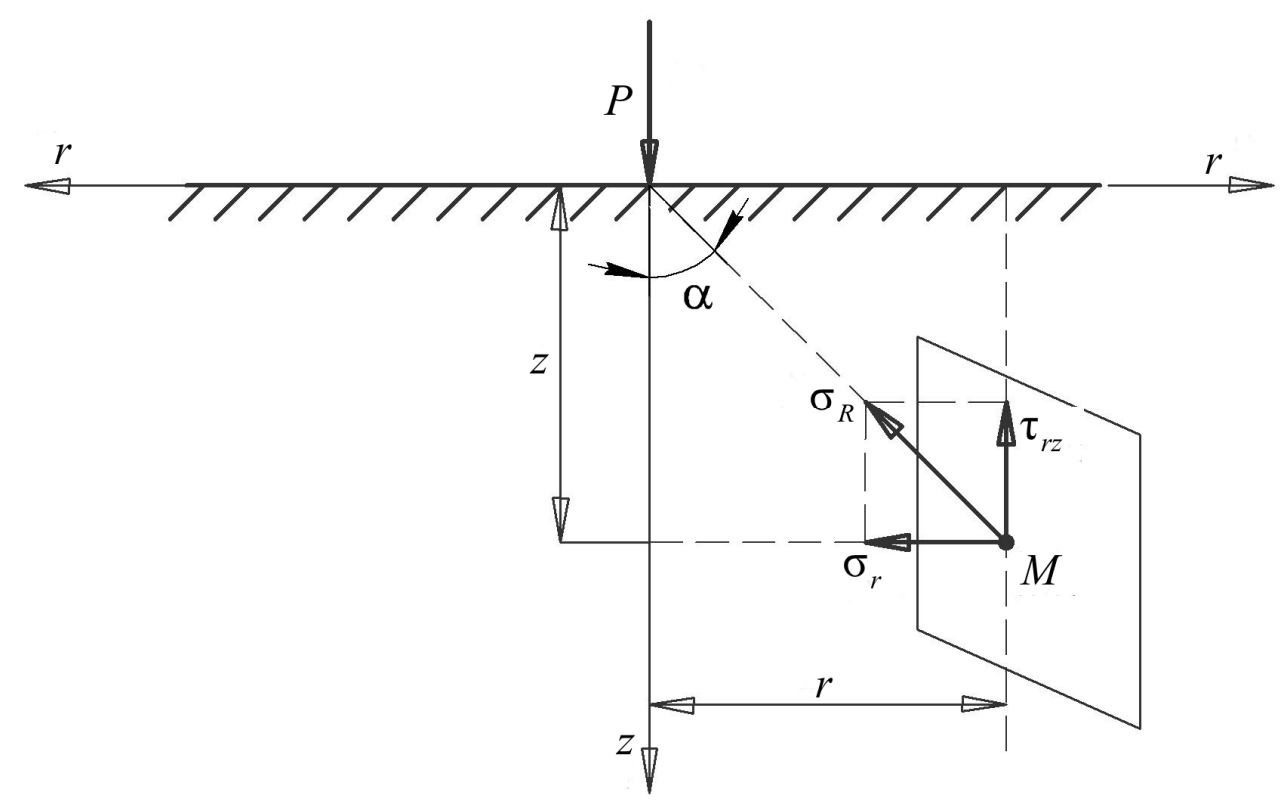

Рис. 2.7. Схема к проверке радиальности напряженного состояния в полупространстве от действия сосредоточенной силы

Таким образом, истинное напряженное состояние в полупространстве от сосредоточенной силы может быть определено, если известна функция радиального напряжения. Чтобы убедиться в этом, зададимся, пока без дополнительных условий, функцией радиального распределения напряжений в полупространстве в виде 


$$
\sigma_{R}=A \frac{z}{R^{3}}
$$

и определим все компоненты напряжений в декартовой системе координат. С этой целью выделим в окрестности какой-либо точки $M$ $(x, y, z)$ элементарный тетраэдр, в котором грань $a b c$ является нормальной к радиус-вектору $R$, проведенному в точку приложения внешней силы, а три остальные грани параллельны координатным плоскостям (рис. 2.8).

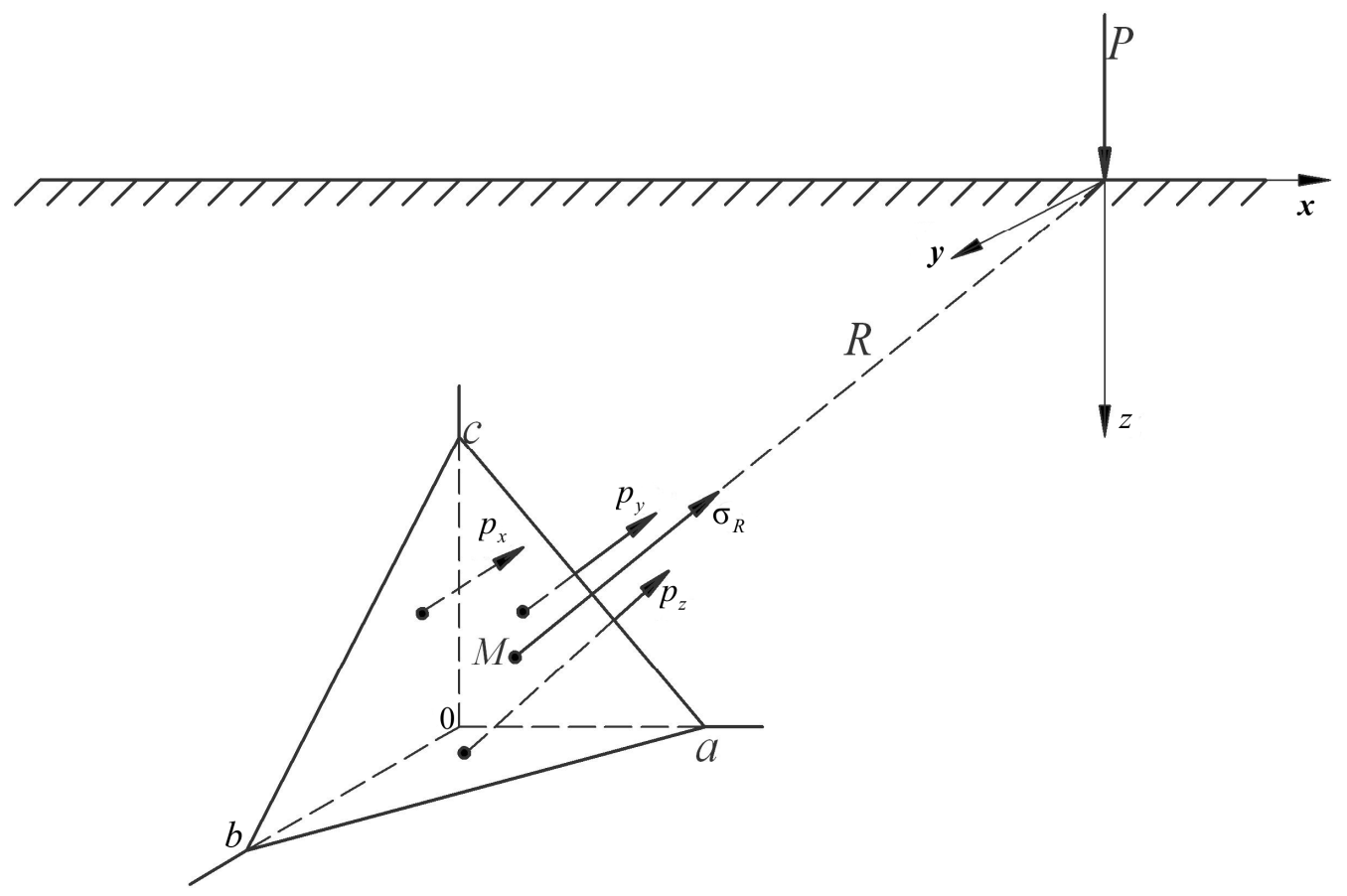

Рис. 2.8. Схема к определению компонентов напряжений в полупространстве от действия сосредоточенной силы при радиальном напряженном состоянии

По площадке $a b c$ действует полное напряжение $\sigma_{R}$. Полные напряжения в остальных площадках тетраэдра будут:

площадка $b o c$

$$
p_{x}=\sigma_{R} \cos (\widehat{x-R})=\sigma_{R} \frac{x}{R}
$$

площадка аос

$$
p_{y}=\sigma_{R} \cos (\widehat{y-R})=\sigma_{R} \frac{y}{R}
$$


площадка $a o b$

$$
p_{z}=\sigma_{R} \cos (\widehat{z-R})=\sigma_{R} \frac{z}{R}
$$

Для определения компонентов напряженного состояния разложим полные напряжения в каждой из трех ортогональных площадок на нормальное и два касательных. При этом следует учесть, что полное напряжение в каждой площадке направлено в точку приложения внешней силы, т. е. совпадает с направлением радиус-вектора $R$.

Компоненты напряжения в плоскости уоz:

$$
\begin{aligned}
& \sigma_{x}=p_{x} \cos (\widehat{R-x})=p_{x} \frac{x}{R}=\sigma_{R} \frac{x}{R} \frac{x}{R}=A \frac{z}{R^{3}} \frac{x^{2}}{R^{2}}=A \frac{x^{2} z}{R^{5}} ; \\
& \tau_{y x}=p_{x} \cos (\widehat{R-y})=p_{x} \frac{y}{R}=\sigma_{R} \frac{x}{R} \frac{y}{R}=A \frac{z}{R^{3}} \frac{x y}{R^{2}}=A \frac{x y z}{R^{5}} ; \\
& \tau_{z x}=p_{x} \cos (\widehat{R-z})=p_{x} \frac{z}{R}=\sigma_{R} \frac{x}{R} \frac{z}{R}=A \frac{z}{R^{3}} \frac{x z}{R^{2}}=A \frac{x z^{2}}{R^{5}},
\end{aligned}
$$

компоненты напряжений в плоскости zox:

$$
\begin{aligned}
& \sigma_{y}=p_{y} \cos (\widehat{R-y})=p_{y} \frac{y}{R}=\sigma_{R} \frac{y}{R} \frac{y}{R}=A \frac{z}{R^{3}} \frac{y^{2}}{R^{2}}=A \frac{y^{2} z}{R^{5}} ; \\
& \tau_{z y}=p_{y} \cos (\widehat{R-z})=p_{y} \frac{z}{R}=\sigma_{R} \frac{y}{R} \frac{z}{R}=A \frac{z}{R^{3}} \frac{y z}{R^{2}}=A \frac{y z^{2}}{R^{5}} ; \\
& \tau_{x y}=p_{y} \cos (\widehat{R-x})=p_{y} \frac{x}{R}=\sigma_{R} \frac{y}{R} \frac{x}{R}=A \frac{z}{R^{3}} \frac{x y}{R^{2}}=A \frac{x y z}{R^{5}},
\end{aligned}
$$

компоненты напряжений в плоскости хоy:

$$
\sigma_{z}=p_{z} \cos (\widehat{R-z})=p_{z} \frac{y}{R}=\sigma_{R} \frac{z}{R} \frac{z}{R}=A \frac{z}{R^{3}} \frac{z^{2}}{R^{2}}=A \frac{z^{3}}{R^{5}}
$$




$$
\begin{aligned}
& \tau_{x z}=p_{z} \cos (\widehat{R-x})=p_{z} \frac{x}{R}=\sigma_{R} \frac{x}{R} \frac{z}{R}=A \frac{z}{R^{3}} \frac{x z}{R^{2}}=A \frac{x z^{2}}{R^{5}} \\
& \tau_{y z}=p_{z} \cos (\widehat{R-y})=p_{z} \frac{y}{R}=\sigma_{R} \frac{y}{R} \frac{z}{R}=A \frac{z}{R^{3}} \frac{y z}{R^{2}}=A \frac{y z^{2}}{R^{5}} .
\end{aligned}
$$

Коэффициент $A$ в компонентах напряжений (2.29)-(2.37) определим из условий равновесия. Для этого проведем полусферическое сечение любым радиусом с центром в точке приложения сосредоточенной силы $P$ (рис. 2.9).

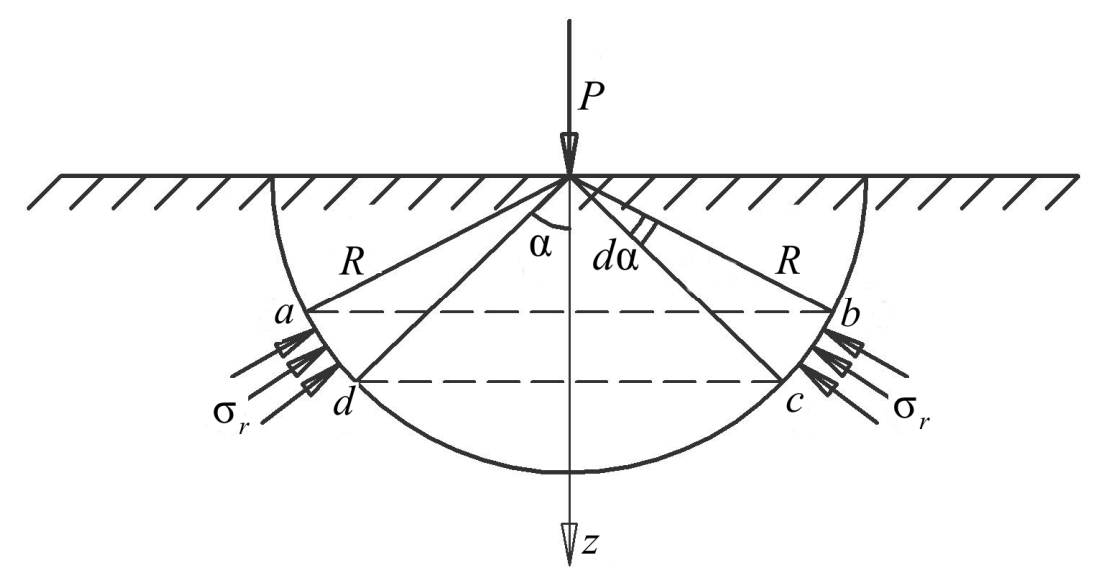

Рис. 2.9. Схема для определения коэффициента пропорциональности $A$ для функции давления (2.28) в полупространстве от действия сосредоточенной силы

По всей поверхности полусферы будут действовать сжимающие радиальные напряжения, величину которых запишем в виде

$$
\sigma_{R}=A \frac{\cos \alpha}{R^{2}}
$$

Для элементарного сферического пояса $a b c d$, отвечающего малому центральному углу $d \alpha$, величину этих напряжений будем считать одинаковой.

Составим уравнение равновесия полусферы:

$$
\int_{0}^{\frac{\pi}{2}} \sigma_{R} \cos \alpha \cdot d F=P,
$$


где $d F$ - поверхность элементарного сферического пояса $a b c d$,

$$
d F=2 \pi(R \sin \alpha)(R d \alpha) z
$$

Выполняем интегрирование $\sigma_{R}$ по всей полусфере:

$$
\begin{gathered}
\int_{0}^{\frac{\pi}{2}} A \frac{\cos ^{2} \alpha}{R^{2}} 2 \pi(R \sin \alpha) \cdot R d \alpha=P \\
A 2 \pi \int_{0}^{\frac{\pi}{2}} \cos ^{2} \alpha \sin \alpha \cdot d \alpha=P \\
\left.A 2 \pi \frac{\cos ^{3} \alpha}{3}\right|_{0} ^{\frac{\pi}{2}}=P
\end{gathered}
$$

а после подстановки пределов

$$
A 2 \pi \frac{1}{3}=P
$$

откуда:

$$
A=\frac{3 P}{2 \pi} .
$$

Окончательно значение радиального напряжения в любой точке полупространства будет

$$
\sigma_{R}=\frac{3 P}{2 \pi} \frac{\cos \alpha}{R^{2}}=\frac{3 P}{2 \pi} \frac{z}{R^{3}} .
$$

Другой возможный способ определения коэффициента $A$ : проинтегрировать напряжения $\sigma_{z}$ по любой горизонтальной плоскости и приравнять сумму внешней силе $P$. Результат получим один и тот же. 
Таким образом, следует считать доказанным, что компоненты напряженного состояния в упругом полупространстве от действия нормальной к его поверхности силы Р для любой изотропной однородной среды будут:

$$
\begin{aligned}
\sigma_{x} & =\frac{3 P}{2 \pi} \frac{x^{2} z}{R^{5}} \\
\sigma_{y} & =\frac{3 P}{2 \pi} \frac{y^{2} z}{R^{5}} \\
\sigma_{z} & =\frac{3 P}{2 \pi} \frac{z^{3}}{R^{5}} \\
\tau_{x y} & =\frac{3 P}{2 \pi} \frac{x y z}{R^{5}} \\
\tau_{y z} & =\frac{3 P}{2 \pi} \frac{y z^{2}}{R^{5}} \\
\tau_{z x} & =\frac{3 P}{2 \pi} \frac{x z^{2}}{R^{5}} .
\end{aligned}
$$

\section{2. Решение Фламана}

Нормально к поверхности упругого полупространства вдоль бесконечной линии равномерно распределена погонная сила $P(\mathrm{H} / \mathrm{M})$. Это задача плоского деформирования полупространства. Она решена Фламаном в 1892 г. [7].

Компоненты напряженного состояния и перемещений запишем в виде $[2 ; 4 ; 6 ; 8 ; 9]$

$$
\sigma_{x}=\frac{2 P}{\pi} \frac{x^{2} z}{\left(x^{2}+z^{2}\right)^{2}}
$$




$$
\begin{aligned}
& \sigma_{z}=\frac{2 P}{\pi} \frac{z^{3}}{\left(x^{2}+z^{2}\right)^{2}} \\
& \tau_{x z}=\frac{2 P}{\pi} \frac{x z^{2}}{\left(x^{2}+z^{2}\right)^{2}} \\
& \sigma_{y}=v \frac{2 P}{\pi} \frac{z}{\left(x^{2}+z^{2}\right)},
\end{aligned}
$$

или

$$
\sigma_{y}=\frac{P}{\pi} \frac{z}{\left(x^{2}+z^{2}\right)}-(1-v) \frac{P}{\pi} \frac{z}{x^{2}+z^{2}}
$$

первый инвариант напряженного состояния

$$
I_{1}=\frac{2 P}{\pi}(1+v) \frac{z}{x^{2}+z^{2}}
$$

или

$$
I_{1}=\frac{3 P}{\pi} \frac{z}{x^{2}+z^{2}}-\frac{1-2 v}{3} \frac{3 P}{\pi} \frac{z}{x^{2}+z^{2}}
$$

компонент горизонтального перемещения

$$
U=-\frac{P(1+v)(1-2 v)}{\pi E} \varphi+\frac{P(1+v)}{\pi E} \frac{x z}{x^{2}+z^{2}} ;
$$

компонент вертикального перемещения

$$
W=-\frac{2 P\left(1-v^{2}\right)}{\pi E}\left(x^{2}+z^{2}\right)^{1 / 2}-\frac{2 P(1+v)}{\pi E} \frac{z^{2}}{x^{2}+z^{2}} .
$$

Для удобства дальнейшего анализа здесь компонент напряженного состояния $\sigma_{y}$ в плоскости деформации и первый инвариант напряженного состояния $I_{1}$ записаны в двух видах - соответственно 
(2.49), (2.50) и (2.51) (2.52). Записи (2.49) и (2.51) являются общепринятыми, а в записях (2.50) и (2.52) выделены части компонентов напряжений, не зависящих от коэффициента Пуассона (первые члены в (2.50) и (2.52)) и зависимых от него (вторые члены в (2.50) и (2.52)).

В формуле (2.53) угол $\varphi$ определяется по схеме, приведенной на рис. 2.10, на котором величина

$$
R=\left(x^{2}+z^{2}\right)^{1 / 2}
$$

представляет радиус-вектор, т. е. расстояние от линии распределения внешней нагрузки $P$ до рассматриваемой точки $M(x, z)$.

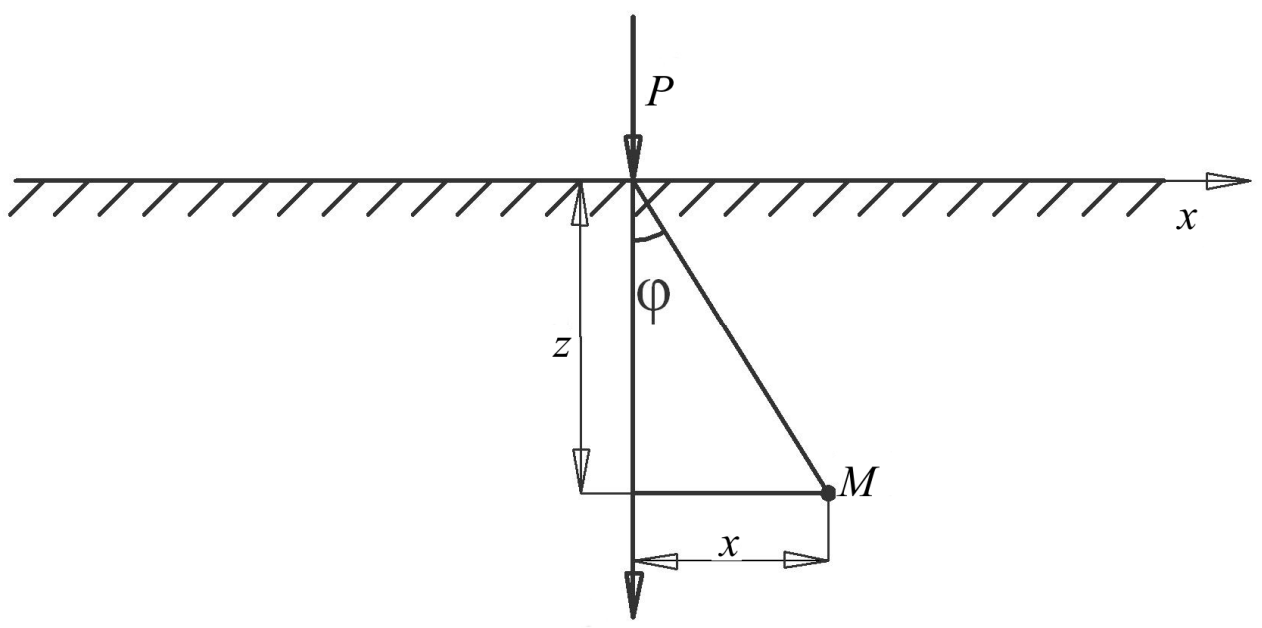

Рис. 2.10. Схема обозначения угла $\varphi$ в (2.53) при определении горизонтальных перемещений полупространства в решении Фламана

В целях установления взаимной связи решений Буссинеска и Фламана попробуем получить компоненты напряженного состояния (2.46)-(2.50) из (2.1)-(2.7) путем предельного перехода. Для этого будем считать, что на поверхности упругого полупространства вдоль оси $y$ равномерно распределена внешняя нагрузка $P(\mathrm{H} / \mathrm{M})$ в пределах от $y=-\infty$ до $y=+\infty$.

Интегрирование компонента $\sigma_{x}(2.1)$

$$
\sigma_{x}=\frac{3 P}{2 \pi} 2 \int_{0}^{\infty} \frac{x^{2} z d y}{\left(x^{2}+y^{2}+z^{2}\right)^{5 / 2}}+\frac{3 P}{2 \pi} \frac{(1-2 v)}{3} \times
$$




$$
\begin{aligned}
& \times 2 \int_{0}^{\infty} \frac{1}{\left(x^{2}+y^{2}+z^{2}\right)^{1 / 2}\left[\left(x^{2}+y^{2}+z^{2}\right)^{1 / 2}+z\right]} \\
& -\frac{\left[2\left(x^{2}+y^{2}+z^{2}\right)^{1 / 2}+z\right] y^{2}}{\left(x^{2}+y^{2}+z^{2}\right)^{3 / 2}\left[\left(x^{2}+y^{2}+z^{2}\right)+z\right]^{2}} d y-\frac{3 P}{2 \pi} \frac{(1-2 v)}{3} 2 \int_{0}^{\infty} \frac{z d y}{\left(x^{2}+y^{2}+z^{2}\right)^{3 / 2}}= \\
& =\left.\frac{3 P}{\pi} \frac{x^{2} z}{\left(x^{2}+z^{2}\right)^{2}}\left[\frac{y}{\left(x^{2}+y^{2}+z^{2}\right)^{1 / 2}}-\frac{y^{3}}{3\left(x^{2}+y^{2}+z^{2}\right)^{3 / 2}}\right]\right|_{0} ^{\infty}+\frac{P}{\pi}(1-2 v) \times \\
& \times\left.\frac{\left[y^{2} z-x^{2}\left(x^{2}+y^{2}+z^{2}\right)^{1 / 2}+z^{3}-z^{2}\left(x^{2}+y^{2}+z^{2}\right)^{1 / 2}+2 x^{2} z\right]}{\left(x^{2}+y^{2}+z^{2}\right)^{1 / 2}\left(x^{2}+z^{2}\right)\left(x^{2}+y^{2}\right)}\right|_{0} ^{\infty}- \\
& -\left.\frac{P}{\pi}(1-2 v) \frac{z}{\left(x^{2}+z^{2}\right)} \frac{y}{\left(x^{2}+y^{2}+z^{2}\right)^{1 / 2}}\right|_{0} ^{\infty}=\frac{3 P}{\pi} \frac{x^{2} z}{\left(x^{2}+z^{2}\right)^{2}} \frac{2}{3}+\frac{P}{\pi}(1-2 v) \times \\
& \times \frac{z}{\left(x^{2}+z^{2}\right)}-\frac{P}{\pi}(1-2 v) \frac{z}{\left(x^{2}+z^{2}\right)}, \quad \sigma_{x}=\frac{2 P}{\pi} \frac{x^{2} z}{\left(x^{2}+z^{2}\right)^{2}},
\end{aligned}
$$

что совпадает с (2.46).

Интегрирование компонента $\sigma_{y}(2.2)$

$$
\begin{aligned}
\sigma_{y}=\frac{3 P}{2 \pi} 2 \int_{0}^{\infty} \frac{y^{2} z d y}{\left(x^{2}+y^{2}+z^{2}\right)^{5 / 2}}+ & \frac{3 P}{2 \pi} \frac{(1-2 v)}{3} \times \\
& \times 2 \int_{0}^{\infty}\left\{\frac{1}{\left(x^{2}+y^{2}+z^{2}\right)^{1 / 2}\left[\left(x^{2}+y^{2}+z^{2}\right)^{1 / 2}+z\right]}-\right.
\end{aligned}
$$




$$
\begin{aligned}
& \left.-\frac{\left[2\left(x^{2}+y^{2}+z^{2}\right)^{1 / 2}+z\right] y^{2}}{\left(x^{2}+y^{2}+z^{2}\right)^{3 / 2}\left[\left(x^{2}+y^{2}+z^{2}\right)+z\right]^{2}}\right\} \times \\
& \times d y-\frac{3 P}{2 \pi} \frac{(1-2 v)}{3} 2 \int_{0}^{\infty} \frac{z d y}{\left(x^{2}+y^{2}+z^{2}\right)^{3 / 2}}= \\
& =\left.\frac{3 P z}{\pi} \frac{1}{3\left(x^{2}+z^{2}\right)} \frac{y}{\left(x^{2}+y^{2}+z^{2}\right)^{1 / 2}}\right|_{0} ^{\infty}-\left.\frac{P(1-2 v)}{\pi} \frac{\left[\left(x^{2}+y^{2}+z^{2}\right)^{1 / 2}\right]}{\left(x^{2}+y^{2}+z^{2}\right)\left(x^{2}+y^{2}\right)}\right|_{0} ^{\infty}- \\
& -\left.\frac{P(1-2 v)}{\pi} \frac{z}{\left(x^{2}+z^{2}\right)} \frac{y}{\left(x^{2}+y^{2}+z^{2}\right)^{1 / 2}}\right|_{0} ^{\infty}= \\
& =\frac{P}{\pi} \frac{z}{\left(x^{2}+z^{2}\right)}-0-\frac{P(1-2 v)}{\pi} \frac{z}{\left(x^{2}+z^{2}\right)}= \\
& =\frac{P}{\pi}(1-1+2 v) \frac{z}{\left(x^{2}+z^{2}\right)}, \quad \sigma_{y}=v \frac{2 P}{\pi} \frac{z}{\left(x^{2}+z^{2}\right)},
\end{aligned}
$$

что совпадает с (2.49).

Интегрирование компонента $\sigma_{z}(2.3)$ :

$$
\begin{aligned}
& \sigma_{z}=\frac{3 P}{2 \pi} 2 \int_{0}^{\infty} \frac{z^{3} d y}{\left(x^{2}+y^{2}+z^{2}\right)^{5 / 2}}=\frac{3 P z^{3}}{\pi\left(x^{2}+z^{2}\right)^{2}} \times \\
& \times\left[\frac{y}{\left(x^{2}+y^{2}+z^{2}\right)^{1 / 2}}-\frac{y^{3}}{3\left(x^{2}+y^{2}+z^{2}\right)^{3 / 2}}\right]_{0}^{\infty}=\frac{3 P z^{3}}{\pi\left(x^{2}+z^{2}\right)^{2}}\left(1-\frac{1}{3}\right),
\end{aligned}
$$




$$
\sigma_{z}=\frac{2 P z^{3}}{\pi\left(x^{2}+z^{2}\right)^{2}}
$$

что совпадает с (2.47).

Интегрирование компонента $\tau_{x z}(2.6)$ :

$$
\tau_{x z}=\frac{3 P}{\pi} \frac{x z^{2}}{\left(x^{2}+z^{2}\right)^{2}} \times
$$

$$
\begin{gathered}
\times\left.\left[\frac{y}{\left(x^{2}+y^{2}+z^{2}\right)^{\frac{1}{2}}}-\frac{y^{3}}{3\left(x^{2}+y^{2}+z^{2}\right)}\right]\right|_{0} ^{\infty}=\frac{3 P}{\pi} \frac{x z^{2}}{\left(x^{2}+z^{2}\right)^{2}} \frac{2}{3}, \\
\tau_{x z}=\frac{2 P}{\pi} \frac{x z^{2}}{\left(x^{2}+z^{2}\right)^{2}},
\end{gathered}
$$

что совпадает с (2.48).

Поскольку функции (2.5) и (2.6) нечетные относительно координаты $y$, то их интегралы в пределах от $y=-\infty$ до $y=+\infty$ сходятся к нулю, поэтому $\tau_{x y}=\tau_{y z}=0$.

Интегрирование первого инварианта напряженного состояния $I_{1}(2.7)$ :

$$
\begin{gathered}
I_{1}=(1+v) \frac{P}{\pi} 2 \int_{0}^{\infty} \frac{z d y}{\left(x^{2}+y^{2}+z^{2}\right)^{3 / 2}}=\left.(1+v) \frac{2 P}{\pi} \frac{z}{\left(x^{2}+z^{2}\right)} \frac{y}{\left(x^{2}+y^{2}+z^{2}\right)^{1 / 2}}\right|_{0} ^{\infty}, \\
I_{1}=(1+v) \frac{2 P z}{\left(x^{2}+z^{2}\right)},
\end{gathered}
$$

что совпадает с (2.51).

Таким образом, путем предельного перехода от напряженного состояния в решении Буссинеска мы получили напряженное состояние в решении Фламана, что подтверждает взаимную связь этих решений. 
Вероятно, что компоненты перемещений (2.15), (2.16) и (2.53), (2.54) также взаимосвязаны между собой, по этой причине и недостатки в рассматриваемом решении имеют близкий характер. Остановимся на некоторых из них.

От коэффициента Пуассона $v$ зависят уже не три, а только один компонент напряжения $\sigma_{y}$ в плоскости деформации, который не оказывает влияния ни на деформации, ни на перемещения, но влияет на величину первого инварианта напряженного состояния, снижая его интенсивность (2.49). Уточним, что зависимость компонента $\sigma_{y}$ от коэффициента Пуассона вытекает из обобщенного физического закона.

Горизонтальные перемещения поверхности, как это следует из (2.50) при $z=0$, будут постоянные по величине на всей ограничивающей полупространство поверхности и направлены к линии приложения сжимающей внешней нагрузки и от нее в случае растягивающей нагрузки [8]:

$$
U(x, 0)_{\substack{x<0 \\ x>0}}= \pm \frac{(1+v)(1-2 v) P}{2 E}
$$

Соответствующая эпюра качественно показана на рис. 2.11, из которого следует, что на линии распределения внешней нагрузки имеется разрыв горизонтальных перемещений. По поводу этого разрыва следует отметить, что к нему приведут любые горизонтальные перемещения поверхности, отличные от нуля.

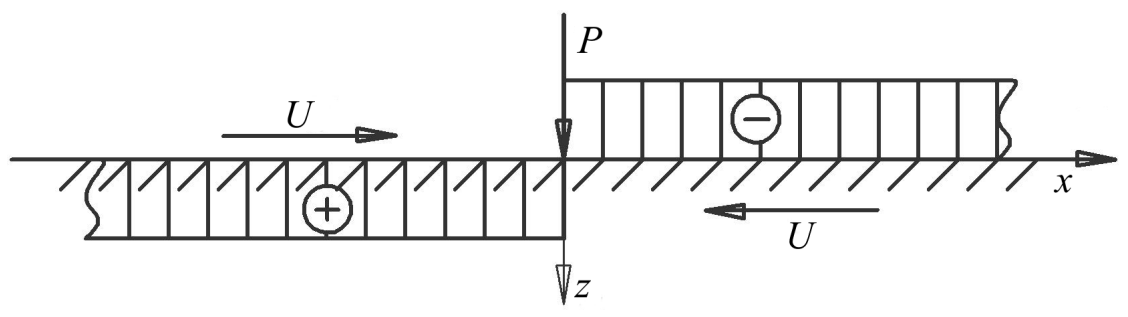

Рис. 2.11. Качественная эпюра горизонтальных перемещений точек поверхности полупространства от равномерно распределенной вдоль бесконечной линии нагрузки (решение Фламана)

Как и в рассмотренном выше решении Буссинеска, в этом решении также наблюдается несимметричное изменение деформирован- 
ного положения поверхности полупространства при симметричном изменении направления действия внешней нагрузки на противоположное, что не может быть подтверждено кинематикой деформирования.

Горизонтальные перемещения в упругом полупространстве при плоском его деформировании линейной нагрузкой определяются зависимостью (2.53). Для удобства анализа запишем ее в виде [2]

$$
U=\frac{P(1+v)}{\pi E}[-(1-2 v) \varphi+\sin \varphi \cos \varphi] .
$$

Рассматривая выражение в квадратных скобках, видим, что горизонтальные перемещения в упругом полупространстве при $z$, отличном от нуля, меняют свое направление на противоположное при некотором удалении от оси $z$. Подобно решению Буссинеска, в котором можно выделить коническую область с вершиной под приложенной силой, в данном решении существует клиновидная область с вершиной под линией распределения нагрузки, в пределах которой горизонтальные перемещения при сжимающей нагрузке направлены от оси $z$, а за ее пределами - к оси $z$ (рис. 2.12). Уравнение этой области можно представить в виде $(1-2 v) \theta=\sin \theta$, в котором $\theta$ составляет угол при вершине клина, он зависит от коэффициента Пуассона.

Предельные значения этого угла будут:

$$
\begin{gathered}
v=0, \quad \theta_{\text {min }}=0 \\
v=0,5, \quad \theta_{\text {max }}=\pi .
\end{gathered}
$$

На рис. 2.12 приведена качественная эпюра горизонтальных перемещений на некоторой глубине $z$. При бесконечном удалении от оси $z$ эти перемещения стремятся к конечному значению:

$$
x \rightarrow \infty, U=-P \frac{(1+v)(1-2 v)}{2 E} .
$$

Для случая, когда коэффициент Пуассона $v=0,5$, горизонтальные перемещения во всем полупространстве при сжимающей внешней нагрузке будут одного знака - растягивающие, а на поверхности они 
будут отсутствовать. Такие перемещения не противоречат кинематике деформирования.

Путем предельного перехода от напряженного состояния в данной задаче мы должны получить полупространство, загруженное равномерно распределенной нагрузкой $p\left(\mathrm{H} / \mathrm{M}^{2}\right)$ по всей его поверхности. С этой целью воспользуемся напряженным состоянием полупространства от действия равномерно распределенной нагрузки по бесконечной полосе шириной $2 a$ [8], переход от которого к напряженному состоянию при загружении всей поверхности полупространства очевиден.

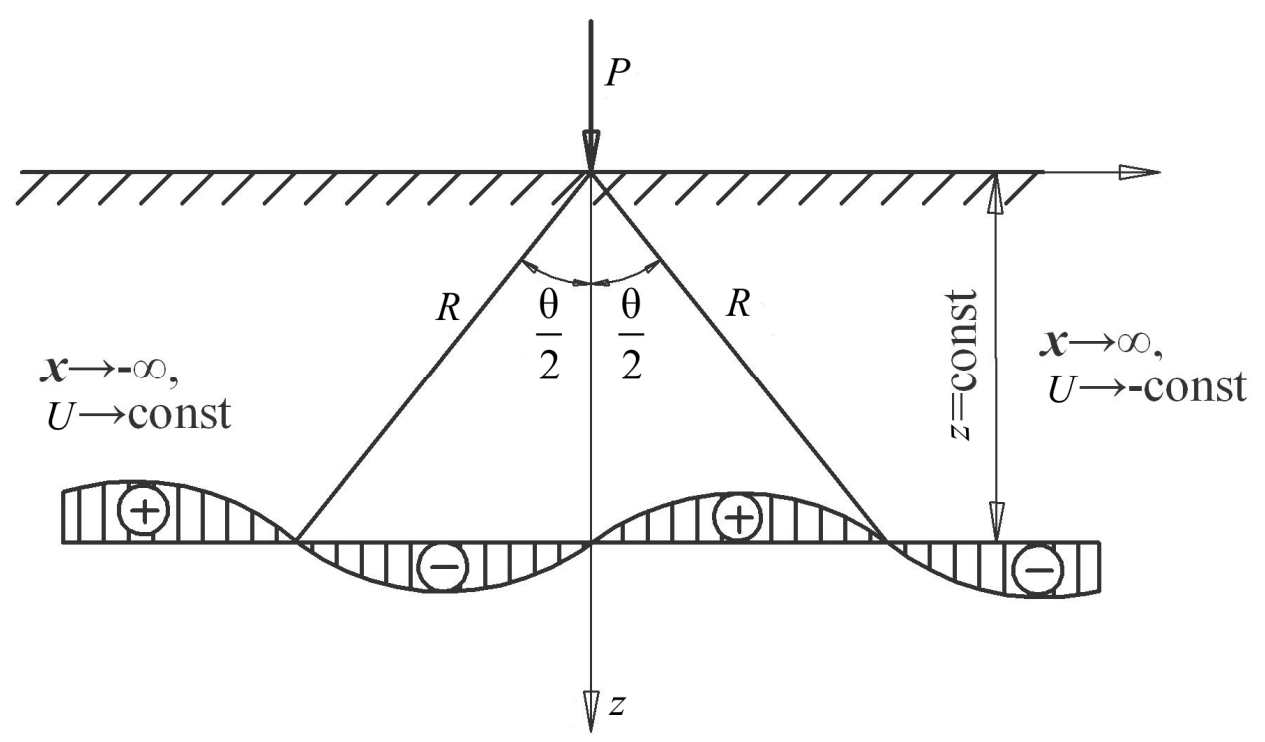

Рис. 2.12. Качественная эпюра горизонтальных смещений

точек полупространства в плоскости $z=$ const от нагрузки, равномерно распределенной вдоль бесконечной линии на поверхности (решение Фламана)

Компоненты напряжений, вызванные полосовой равномерно распределенной нагрузкой, будут:

$$
\begin{aligned}
& \sigma_{x}=\frac{p}{\pi}\left(\operatorname{arctg} \frac{a-x}{z}+\operatorname{arctg} \frac{a+x}{z}\right)+\frac{2 p a z\left(x^{2}-z^{2}-a^{2}\right)}{\pi\left[\left(x^{2}+z^{2}-a^{2}\right)+4 a^{2} z^{2}\right]} ;(2.55) \\
& \sigma_{z}=\frac{p}{\pi}\left(\operatorname{arctg} \frac{a-x}{z}+\operatorname{arctg} \frac{a+x}{z}\right)-\frac{2 p a z\left(x^{2}-z^{2}-a^{2}\right)}{\pi\left[\left(x^{2}+z^{2}-a^{2}\right)+4 a^{2} z^{2}\right]} ;(2.56)
\end{aligned}
$$




$$
\begin{gathered}
\tau_{x z}=\frac{4 p a x z^{2}}{\pi\left[\left(x^{2}+z^{2}-a^{2}\right)+4 a^{2} z^{2}\right]} ; \\
\sigma_{y}=v\left(\sigma_{x}+\sigma_{z}\right)=v \frac{2 p}{\pi}\left(\operatorname{arctg} \frac{a-x}{z}+\operatorname{arctg} \frac{a+x}{z}\right),
\end{gathered}
$$

или

$$
\begin{aligned}
\sigma_{y}=\frac{p}{\pi}\left(\operatorname{arctg} \frac{a-x}{z}+\operatorname{arctg} \frac{a+x}{z}\right)- \\
\\
\quad-\frac{p}{\pi}(1-2 v)\left(\operatorname{arctg} \frac{a-x}{z}+\operatorname{arctg} \frac{a+x}{z}\right)
\end{aligned}
$$

первый инвариант напряженного состояния

$$
I_{1}=(1+v) \frac{p}{\pi}\left(\operatorname{arctg} \frac{a-x}{z}+\operatorname{arctg} \frac{a+x}{z}\right)
$$

или

$$
\begin{aligned}
I_{1}=\frac{3 p}{\pi}\left(\operatorname{arctg} \frac{a-x}{z}+\operatorname{arctg} \frac{a+x}{z}\right)- \\
\quad-\frac{(1-2 v)}{3} \frac{3 p}{\pi}\left(\operatorname{arctg} \frac{a-x}{z}+\operatorname{arctg} \frac{a+x}{z}\right) .
\end{aligned}
$$

В формулах (2.59) и (2.61) для компонента напряжения $\sigma_{y}$ и первого инварианта напряжений $I_{1}$ выделены отдельно их части, не зависимые от коэффициента Пуассона и зависимые от него.

Предельный переход от (2.55)-(2.60) путем а $\rightarrow \infty$ дает

$$
\sigma_{x}=\sigma_{z}=p ; \quad \sigma_{y}=v 2 p ; \quad \tau_{z x}=0 ; \quad I_{1}=2 p(1+v)
$$


то есть получаем явно бессмысленный результат, т. к. $\sigma_{x}$ и $\sigma_{y}$ не равны между собой.

Задача по определению напряженного состояния полупространства, вся поверхность которого загружена равномерно распределенной нагрузкой $p$, может быть решена и другим путем, исходя из обобщенных физических зависимостей. Для этого заменим в первых двух уравнениях (1.12) вертикальный компонент $\sigma_{z}=p$ и ввиду отсутствия горизонтальных перемещений и деформаций получим

$$
\left.\begin{array}{l}
\varepsilon_{x}=\frac{1}{E}\left[\sigma_{x}-v\left(\sigma_{y}+p\right)\right] ; \\
\varepsilon_{y}=\frac{1}{E}\left[\sigma_{y}-v\left(\sigma_{x}+p\right)\right] .
\end{array}\right\}
$$

Решение системы уравнений (2.63) дает

$$
\sigma_{x}=\sigma_{y}=\frac{v}{1-v} p=k_{k} p
$$

где $k_{k}$ - коэффициент, который в механике грунтов называют коэффициентом бокового давления, или геостатическим коэффициентом $[8 ; 9 ; 13]$. Он определяет соотношение главных напряжений в условиях отсутствия боковых деформаций упругой среды.

Как видим, два подхода к решению одной и той же задачи дают различные результаты, чего в принципе быть не должно.

Причина несовпадения результатов предельного перехода (2.62) и прямого решения (2.64), а также зависимости компонента $\sigma_{y}$ от коэффициента Пуассона при плоском деформировании полупространства скрыта в неточном отражении связи между напряженным и деформированным состояниями существующими физическими зависимостями.

Ответить на вопрос о том, каким же все-таки должно быть напряженное состояние упругого полупространства при загружении его поверхности равномерной нагрузкой, можно очень просто, руководствуясь следующими соображениями.

Поскольку истинное напряженное состояние в изотропном упругом полупространстве от сосредоточенной нормальной к его поверх- 
ности силы $P(\mathrm{H})$ мы уже определили в виде (2.29)-(2.37), то переход к решению, когда на поверхности этого полупространства действует равномерно распределенная вдоль бесконечной линии сила $P(\mathrm{H} / \mathrm{M})$, можно выполнить путем предельного интегрирования компонентов (2.29)-(2.37). Выполним это интегрирование.

Компонент $\sigma_{x}$ :

$$
\begin{aligned}
\sigma_{x}=2 \int_{0}^{\infty} \frac{3 P}{2 \pi} \frac{x^{2} z d y}{\left(x^{2}+y^{2}+z^{2}\right)^{5 / 2}}= & \frac{3 P}{2 \pi} \frac{x^{2} z}{\left(x^{2}+z^{2}\right)^{2}} \times \\
& \times\left[\frac{y}{\left(x^{2}+y^{2}+z^{2}\right)^{1 / 2}}-\frac{y^{3}}{3\left(x^{2}+y^{2}+z^{2}\right)^{3 / 2}}\right] \\
\sigma_{x} & =\frac{2 P}{\pi} \frac{x^{2} z}{\left(x^{2}+z^{2}\right)^{2}} ;
\end{aligned}
$$

компонент $\sigma_{z}$ :

$$
\begin{aligned}
\sigma_{z}=2 \int_{0}^{\infty} \frac{3 P}{2 \pi} \frac{z^{3} d y}{\left(x^{2}+y^{2}+z^{2}\right)^{5 / 2}}= & \frac{3 P}{\pi} \frac{z^{3}}{\left(x^{2}+z^{2}\right)^{2}} \times \\
& \times\left[\frac{y}{\left(x^{2}+y^{2}+z^{2}\right)^{1 / 2}}--\frac{y^{3}}{3\left(x^{2}+y^{2}+z^{2}\right)^{3 / 2}}\right] \\
\sigma_{z} & =\frac{2 P}{\pi} \frac{z^{3}}{\left(x^{2}+z^{2}\right)^{2}} ;
\end{aligned}
$$

компонент $\sigma_{y}$ :

$$
\sigma_{y}=2 \int_{0}^{\infty} \frac{3 P}{2 \pi} \frac{y^{2} z d y}{\left(x^{2}+y^{2}+z^{2}\right)^{5 / 2}}=\left.\frac{3 P}{\pi} \frac{z y^{3}}{3\left(x^{2}+z^{2}\right)^{2}\left(x^{2}+y^{2}+z^{2}\right)^{3 / 2}}\right|_{0} ^{\infty}
$$




$$
\sigma_{y}=\frac{P}{\pi} \frac{z}{x^{2}+z^{2}}
$$

компонент $\tau_{x z}$ :

$$
\begin{aligned}
\tau_{x z}=2 \int_{0}^{\infty} \frac{3 P}{2 \pi} \frac{x z^{2} d y}{\left(x^{2}+y^{2}+z^{2}\right)^{5 / 2}=} & \frac{3 P}{\pi} \frac{x z^{2}}{\left(x^{2}+z^{2}\right)^{2}} \times \\
& \times\left[\frac{y}{\left(x^{2}+y^{2}+z^{2}\right)^{1 / 2}}--\frac{y^{3}}{3\left(x^{2}+y^{2}+z^{2}\right)^{3 / 2}}\right] \mid ; \\
\tau_{x z} & =\frac{2 P}{\pi} \frac{x z^{2}}{\left(x^{2}+z^{2}\right)^{2}} .
\end{aligned}
$$

Поскольку подинтегральные функции (2.34) и (2.37) нечетные относительно координаты $y$, то их интегралы в пределах от $y=-\infty$ до $y=+\infty$ сходятся к нулю. Поэтому имеем:

$$
\begin{gathered}
\tau_{x y}=\int_{-\infty}^{\infty} \frac{3 P}{\pi} \frac{x z y d y}{\left(x^{2}+y^{2}+z^{2}\right)^{5 / 2}}=0 \\
\tau_{y z}=\int_{-\infty}^{\infty} \frac{3 P}{2 \pi} \frac{x z^{2} y d y}{\left(x^{2}+y^{2}+z^{2}\right)^{5 / 2}}=0 .
\end{gathered}
$$

Интегрирование первого инварианта напряжений $I_{1}$ :

$$
\begin{gathered}
I_{1}=2 \int_{0}^{\infty} \frac{3 P}{2 \pi} \frac{z d y}{\left(x^{2}+y^{2}+z^{2}\right)^{3 / 2}}=\left.\frac{3 P}{\pi} \frac{z y}{\left(x^{2}+z^{2}\right)^{2}\left(x^{2}+y^{2}+z^{2}\right)^{1 / 2}}\right|_{0} ^{\infty} ; \\
I_{1}=\frac{3 P}{\pi} \frac{z}{x^{2}+z^{2}} .
\end{gathered}
$$


Решение задачи плоского деформирования упругого полупространства, когда на его поверхности равномерно распределена нагрузка по бесконечной полосе постоянной ширины, получим из формул (2.55)-(2.61), где в компоненте $\sigma_{y}(2.59)$ и первом инварианте напряженного состояния $I_{1}(2.61)$ формально примем $v=0,5$.

Из полученных соотношений следует, что для случая плоского деформирования компонент нормального напряжения $\sigma_{y}$ в плоскости деформации (2.66) равен среднему значению между двумя другими компонентами нормальных напряжений $\sigma_{x}$ и $\sigma_{z}$ в плоскостях, перпендикулярных плоскости деформации. Поэтому в системе напряжений (2.46)-(2.52) не должно быть их зависимостей от коэффициента Пуассона. Компонент $\sigma_{y}(2.49)$ должен быть заменен компонентом (2.67), а первый инвариант напряжений $I_{1}$ (2.51) и (2.52) на (2.69). После этого предельный переход к напряженному полупространству, когда на всей ограничивающей его поверхности будет действовать равномерно распределенная нагрузка интенсивностью $p\left(\mathrm{H} / \mathrm{M}^{2}\right)$, дает следующие результаты.

Интегрирование компонента $\sigma_{x}(2.68)$ :

$$
\sigma_{x}=2 \int_{0}^{\infty} \frac{2 p}{\pi} \frac{z x^{2} d x}{\left(x^{2}+z^{2}\right)^{2}}=\frac{4 p z}{\pi}\left[-\frac{x}{\left(x^{2}+z^{2}\right)}+\frac{1}{2 z} \operatorname{arctg} \frac{x}{z}\right] \prod_{0}^{\infty} ; \quad \sigma_{x}=p .
$$

Интегрирование компонента $\sigma_{z}(2.66)$ :

$$
\sigma_{z}=2 \int_{0}^{\infty} \frac{2 p}{\pi} \frac{z^{3} d x}{\left(x^{2}+z^{2}\right)^{2}}=\left.\frac{4 p z^{3}}{\pi}\left[\frac{x}{2 z^{2}\left(x^{2}+z^{2}\right)}+\frac{1}{2 z^{3}} \operatorname{arctg} \frac{x}{z}\right]\right|_{0} ^{\infty} ; \quad \sigma_{z}=p
$$

Интегрирование компонента $\sigma_{y}(2.67)$ :

$$
\sigma_{y}=2 \int_{0}^{\infty} \frac{p}{\pi} \frac{z d x}{x^{2}+z^{2}}=\left.\frac{2 p z}{\pi} \frac{1}{z} \operatorname{arctg} \frac{x}{z}\right|_{0} ^{\infty} ; \quad \sigma_{y}=p
$$


Поскольку функция $\tau_{z x}(2.68)$ нечетная относительно координаты $x$, то ее интеграл в пределах от $x=-\infty$ до $x=+\infty$ сходится к нулю. В результате получим $\tau_{z x}=0$.

Интегрирование первого инварианта напряжений (2.69)

$$
\begin{gathered}
I_{1}=2 \int_{0}^{\infty} \frac{3 p}{\pi} \frac{z d x}{x^{2}+z^{2}}=\left.\frac{6 p z}{\pi} \frac{1}{z} \operatorname{arctg} \frac{x}{z}\right|_{0} ^{\infty} ; \\
I_{1}=3 p .
\end{gathered}
$$

Таким образом, мы пришли к результату о том, что при загружении всего полупространства равномерной нагрузкой интенсивностью $p\left(\mathrm{H} / \mathrm{M}^{2}\right)$ напряженное состояние его будет однородным, гидростатическим:

$$
\left.\begin{array}{l}
\sigma_{x}=\sigma_{y}=\sigma_{z}=p \\
I_{1}=\sigma_{x}+\sigma_{y}+\sigma_{z}=3 p .
\end{array}\right\}
$$

Теперь решения трех задач для полупространства, когда нормально к его поверхности действуют: сосредоточенная нагрузка $P(\mathrm{H})$; равномерно распределенная по бесконечной линии нагрузка интенсивностью $P(\mathrm{H} / \mathrm{M})$, равномерно распределенная по всей поверхности нагрузка интенсивностью $p\left(\mathrm{H} / \mathrm{M}^{2}\right)$, взаимно связаны: от первой задачи предельным интегрированием получаем решение второй задачи, а от второй задачи предельным интегрированием получаем решение третьей задачи. Все логично и математически обосновано, без ограничений и допущений.

\section{3. Решение Кельвина}

Сила $P$ сосредоточена в некоторой точке упругого пространства. Решение этой задачи получил в 1848 году Кельвин [10]. Приведем компоненты напряженного состояния этой задачи в цилиндрических координатах $[4 ; 11]$ : 


$$
\begin{gathered}
\sigma_{r}=\frac{P}{8 \pi(1-v)}\left[\frac{3 r^{2} z}{\left(r^{2}+z^{2}\right)^{5 / 2}}-(1-2 v) \frac{z}{\left(r^{2}+z^{2}\right)^{3 / 2}}\right] \\
\sigma_{\theta}=-\frac{P(1-2 v)}{8 \pi(1-v)} \frac{z}{\left(r^{2}+z^{2}\right)^{3 / 2}} ; \\
\sigma_{z}=\frac{P}{8 \pi(1-v)}\left[\frac{3 z^{3}}{\left(r^{2}+z^{2}\right)^{5 / 2}}+(1-2 v) \frac{z}{\left(r^{2}+z^{2}\right)^{3 / 2}}\right] \\
\tau_{z r}=\frac{P}{8 \pi(1-v)}\left[\frac{3 r z^{2}}{\left(r^{2}+z^{2}\right)^{5 / 2}}+(1-2 v) \frac{z}{\left(r^{2}+z^{2}\right)^{3 / 2}}\right] .
\end{gathered}
$$

Эта задача имеет скорее теоретическое, познавательное значение, чем практическое. Она играет существенную роль в анализе напряженно-деформированного состояния полупространства и пространства, являясь важным звеном в проявлении взаимозависимости решений задач теории упругости для массивов.

Как видим, напряженное состояние (2.70)-(2.73) зависит от коэффициента Пуассона. В целях анализа этой зависимости запишем рассматриваемое решение для предельного случая, когда упругая характеристика $v=0,5$ и напряженное состояние не будет от нее зависеть:

$$
\begin{aligned}
& \sigma_{r}^{\prime}=\frac{3 P}{4 \pi} \frac{r^{2} z}{\left(r^{2}+z^{2}\right)^{5 / 2}} ; \\
& \sigma_{\theta}^{\prime}=0 ; \\
& \sigma_{z}^{\prime}=\frac{3 P}{4 \pi} \frac{z^{3}}{\left(r^{2}+z^{2}\right)^{5 / 2}} ;
\end{aligned}
$$




$$
\tau_{z r}^{\prime}=\frac{3 P}{4 \pi} \frac{r z^{2}}{\left(r^{2}+z^{2}\right)^{5 / 2}} .
$$

Как видим, компоненты этих напряжений в два раза меньше компонентов напряжений для полупространства при тех же условиях (формулы (2.11)-(2.14), в которых принято $v=0,5)$, и это вполне логично, потому что сила Р в (2.74)-(2.77) равномерно распределяется на два полупространства, одно из которых она сжимает, а другое растягивает.

Разделим теперь напряженное состояние в упругом бесконечном пространстве (2.70)-(2.73) на две части, выделив в каждом компоненте часть напряжения, которая не зависит от коэффициента Пуассона (система (2.74)-(2.77)). Оставшаяся часть будет зависимой от него и определяется по схеме.

$$
\begin{aligned}
& \sigma_{r}^{\prime \prime}=-\sigma_{r}^{\prime}+\sigma_{r} ; \\
& \sigma_{\theta}^{\prime \prime}=-\sigma_{\theta}^{\prime}+\sigma_{\theta} ; \\
& \sigma_{z}^{\prime \prime}=-\sigma_{z}^{\prime}+\sigma_{z} ; \\
& \tau_{r z}^{\prime \prime}=-\tau_{r z}^{\prime}+\tau_{r z} .
\end{aligned}
$$

После подстановки в (2.78)-(2.81) значений соответствующих компонентов напряжения из систем (2.70)-(2.73) и (2.74)-(2.77) имеем:

$$
\begin{gathered}
\sigma_{r}^{\prime \prime}=-\frac{3 P}{4 \pi} \frac{r^{2} z}{\left(r^{2}+z^{2}\right)^{5 / 2}}+\frac{P}{8 \pi(1-v)}\left[\frac{3 r^{2} z}{\left(r^{2}+z^{2}\right)^{5 / 2}}-(1-2 v) \frac{z}{\left(r^{2}+z^{2}\right)^{5 / 2}}\right] \\
\sigma_{r}^{\prime \prime}=-\frac{P(1-2 v)}{8 \pi(1-v)}\left[\frac{3 r^{2} z}{\left(r^{2}+z^{2}\right)^{5 / 2}}+\frac{z}{\left(r^{2}+z^{2}\right)^{3 / 2}}\right]
\end{gathered}
$$




$$
\begin{gathered}
\sigma_{\theta}^{\prime \prime}=-\frac{P(1-2 v)}{8 \pi(1-v)} \frac{z}{\left(r^{2}+z^{2}\right)^{3 / 2}} ; \\
\sigma_{z}^{\prime \prime}=-\frac{3 P}{4 \pi} \frac{z^{3}}{\left(r^{2}+z^{2}\right)^{5 / 2}}+\frac{P}{8 \pi(1-v)}\left[\frac{z^{3}}{\left(r^{2}+z^{2}\right)^{5 / 2}}+(1-2 v) \frac{z}{\left(r^{2}+z^{2}\right)^{3 / 2}}\right] \\
\tau_{r z}^{\prime \prime}=-\frac{3 P}{4 \pi} \frac{r z^{2}}{\left(r^{2}+z^{2}\right)^{5 / 2}}+\frac{P}{8 \pi(1-v)}\left[(1-2 v) \frac{r}{\left(r^{2}+z^{2}\right)^{3 / 2}}+\frac{3 r z^{2}}{\left(r^{2}+z^{2}\right)^{5 / 2}}\right] \\
\sigma_{r z}^{\prime \prime}=-\frac{3(1-2 v)}{8 \pi(1-v)}\left[\frac{\left(r^{2}+z^{2}\right)^{5 / 2}}{8 \pi(1-2 v)}\left[\frac{r}{\left(r^{2}+z^{2}\right)^{3 / 2}}\right]\right.
\end{gathered}
$$

Очевидно, что система полных напряжений (2.70)-(2.73) эквивалентна сумме двух систем напряжений (2.74)-(2.77) и (2.82)-(2.85). Обе части напряженного состояния в отдельности удовлетворяют дифференциальным условиям равновесия (1.8). Однако, если система (2.74)-(2.77) уравновешивает внешнюю силу $P$, так как интегрирование компонента $\sigma_{z}^{\prime}$ по любой горизонтальной плоскости дает силу $0,5 P$, то система (2.82)-(2.85) не уравновешивает ничего, в чем можно убедиться интегрированием добавочной части компонента вертикального напряжения $\sigma_{z}^{\prime \prime}(2.85)$, который зависит от коэффициента Пуассона. С этой целью сначала представим ее в виде

$$
\sigma_{z}^{\prime \prime}=-B \frac{3 z^{3}}{\left(r^{2}+z^{2}\right)^{5 / 2}}+B \frac{z}{\left(r^{2}+z^{2}\right)^{3 / 2}}
$$

где $B$ - постоянный коэффициент, равный $B=\frac{P}{8 \pi} \frac{1-2 v}{1-v}$. 
Выделим в упругом пространстве двумя параллельными бесконечными плоскостями, расположенными на расстояниях $\pm z$ от точки приложения силы $P$, часть его объема (рис. 2.13).
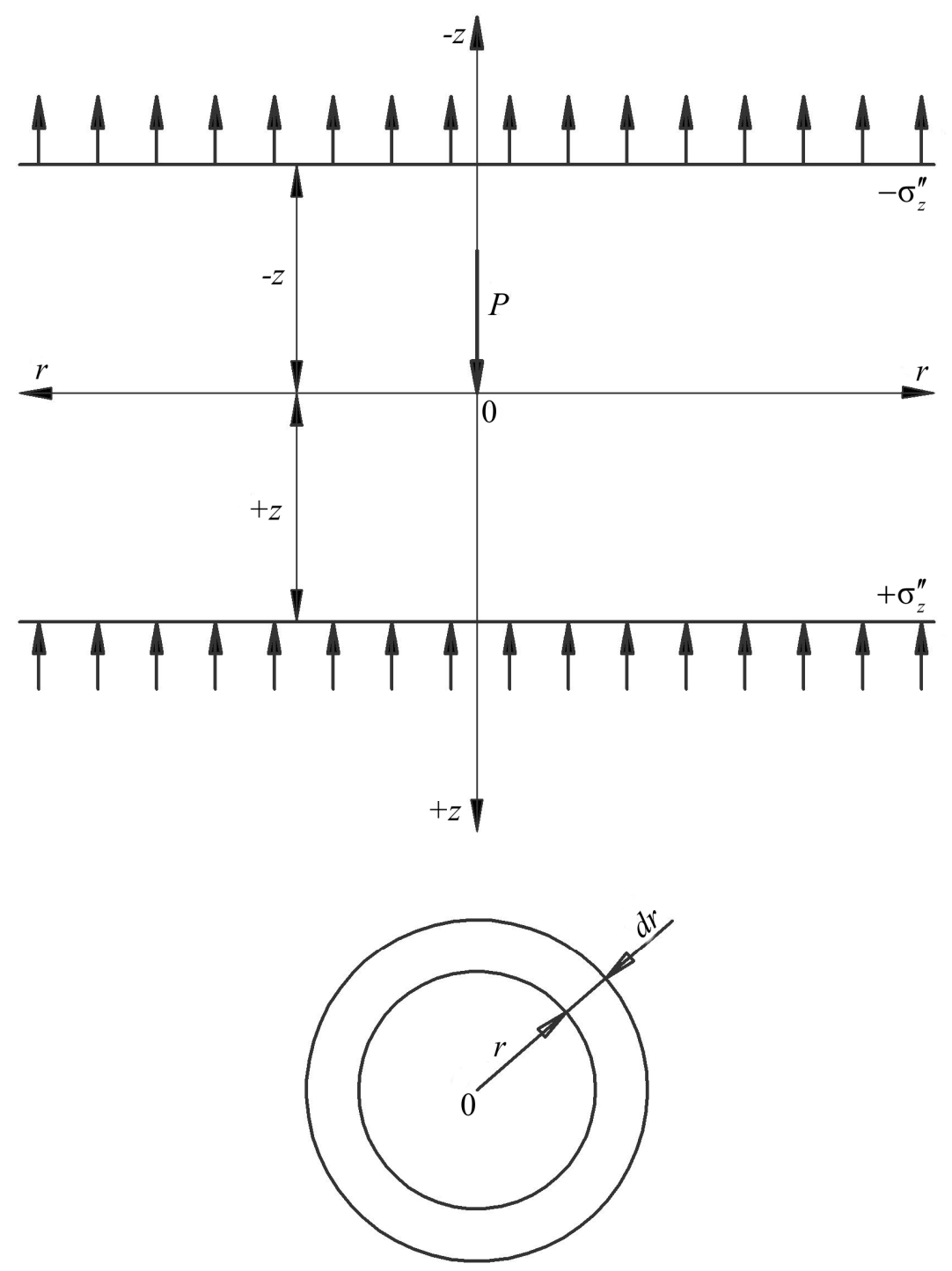

Рис. 2.13. Схема интегрирования компонента $\sigma_{z}^{\prime \prime}(2.84)$ в решении Кельвина

На одной из плоскостей выделим кольцевой элемент $d F$ радиусом $r$ и центром на оси $z$ (см. рис. 2.13):

$$
d F=2 \pi r d r
$$

Составим интегралы для суммирования напряжения $\sigma_{z}^{\prime \prime}$ по всей плоскости: 


$$
\begin{aligned}
& B \int_{0}^{\infty} \frac{3 z^{3} 2 \pi r d r}{\left(r^{2}+z^{2}\right)^{5 / 2}}-B \int_{0}^{\infty} \frac{z 2 \pi r d r}{\left(r^{2}+z^{2}\right)^{3 / 2}}= \\
& =\left.B 6 \pi z^{3}\left[-\frac{1}{3\left(r^{2}+z^{2}\right)^{3 / 2}}\right]\right|_{0} ^{\infty}-B 2 \pi z\left[-\left.\frac{1}{\left(r^{2}+z^{2}\right)^{1 / 2}}\right|_{0} ^{\infty}=\right. \\
& \quad=-B 2 \pi z^{3}\left(0-\frac{1}{z^{3}}\right)+B 2 \pi z\left(0-\frac{1}{z}\right)=-2 \pi B+2 \pi B=0,
\end{aligned}
$$

то есть суммарное давление на эту плоскость от напряжений $\sigma_{z}^{\prime \prime}$ равно нулю. Очевидно, что интегрирование по второй плоскости приведет к такому же результату.

С другой стороны, первые инварианты напряженного состояния рассматриваемых систем соответственно равны:

$$
\begin{gathered}
I_{1}^{\prime}=\sigma_{r}^{\prime}+\sigma_{\theta}^{\prime}+\sigma_{z}^{\prime}=\frac{3 P}{4 \pi}\left[\frac{r^{2} z}{\left(r^{2}+z^{2}\right)}+0+\frac{z^{3}}{\left(r^{2}+z^{2}\right)^{5 / 2}}\right] \\
I_{1}^{\prime}=\frac{3 P}{4 \pi} \frac{z}{\left(r^{2}+z^{2}\right)^{3 / 2}}, \\
I_{1}^{\prime \prime}=\sigma_{r}^{\prime \prime}+\sigma_{\theta}^{\prime \prime}+\sigma_{z}^{\prime \prime}=-\frac{3 P(1-2 v)}{8 \pi(1-v)} \frac{r^{2} z}{\left(r^{2}+z^{2}\right)^{5 / 2}}- \\
\quad \times \frac{z(1-2 v)}{\left(r^{2}+z^{2}\right)^{3 / 2}}-\frac{3 P(1-2 v)}{8 \pi(1-v)} \frac{z^{3}}{\left(r^{2}+z^{2}\right)^{5 / 2}}+\frac{P(1-2 v)}{8 \pi(1-v)} \frac{z(1-2 v)}{\left(r^{2}+z^{2}\right)^{3 / 2}} \times \frac{\left.z z^{2}\right)^{3 / 2}}{8 \pi(1-v)}
\end{gathered}
$$




$$
I_{1}^{\prime \prime}=-\frac{P}{2 \pi} \frac{(1-2 v)}{(1-v)} \frac{z}{\left(r^{2}+z^{2}\right)^{3 / 2}} .
$$

Полностью первый инвариант напряженного состояния запишем в виде

$$
I_{1}=I_{1}^{\prime \prime}+I_{1}^{\prime \prime}=\frac{3 P}{4 \pi} \frac{z}{\left(r^{2}+z^{3}\right)^{3 / 2}}-\frac{2(1-2 v)}{3(1-v)} \frac{3 P}{4 \pi} \frac{z}{\left(r^{2}+z^{2}\right)^{3 / 2}}
$$

или

$$
I_{1}=\frac{3}{4 \pi} \frac{z}{\left(r^{2}+z^{2}\right)^{3 / 2}}\left[P-\frac{2(1-2 v)}{3(1-v)} P\right]
$$

Здесь функция перед квадратными скобками - гармоническая, следовательно, потенциальная; она отвечает конкретным граничным условиям и полностью характеризует интенсивность напряженного состояния в упругом массиве, ее источником является конкретная сила. Но в скобках две силы, действующие в одной и той же точке: сила $P$ и часть этой силы с обратным знаком, уменьшающая интенсивность напряженного состояния в целом. По всем законам их следует алгебраически суммировать, но в задаче наблюдается влияние второй силы на напряженное состояние, а в равновесии она не учитывается, то есть является фиктивной.

Как и в решении Буссинеска, можно показать, что в рассматриваемом решении в общем случае распределение напряжений не является радиальным, поэтому в нем также не выполняется и третий закон механики. Однако существует единственный, частный случай напряженного состояния упругого пространства от действующей внутри его сосредоточенной силы $P$, когда выполняются и третий закон механики, и условия равновесия. Он представлен компонентами (2.74)-(2.77), т. е. при радиальном напряженном состоянии, которое не зависит от коэффициента Пуассона.

Таким образом, мы приходим к выводу о том, что эти две задачи близнецы. Сложив из двух полупространств полное пространство таким способом, чтобы внутри его оказалась сосредоточенная сила $P$, 
и продлив ось $z$ в обе стороны от $-\infty$ до $+\infty$, мы распределяем эту силу в равных частях на две половины пространства: к одной половине приложена сжимающая сила, равная $0,5 P$, а к другой - растягивающая сила такой же величины.

Формально в решения (2.40)-(2.45) следует ввести коэффициент 0,5 , и тогда получим единственное решение задачи о распределении напряжений в однородном упругом пространстве от сосредоточенной силы, которое в декартовой системе координат будет представлено в виде:

$$
\left.\begin{array}{ll}
\sigma_{x}=\frac{3 P}{8 \pi} \frac{x^{2} z}{R^{5}} ; & \sigma_{x y}=\frac{3 P}{8 \pi} \frac{x y z}{R^{5}} ; \\
\sigma_{y}=\frac{3 P}{8 \pi} \frac{y^{2} z}{R^{5}} ; & \sigma_{y z}=\frac{3 P}{8 \pi} \frac{y z^{2}}{R^{5}} ; \\
\sigma_{z}=\frac{3 P}{8 \pi} \frac{z^{3}}{R^{5}} ; & \sigma_{z x}=\frac{3 P}{8 \pi} \frac{x z^{2}}{R^{5}} .
\end{array}\right\}
$$

\section{4. Деформации упругого изменения объема и формы}

Общую деформацию элементарного объема упругой среды в теории упругости делят на два вида - деформацию изменения объема и деформацию изменения формы. Соответственно сформулированы два закона: закон упругого изменения объема (1.17), (1.21), (1.22) и закон упругого изменения формы (1.18)-(1.20).

В законе упругого изменения объема дается величина объемной относительной деформации и принимается, что ее вызывает шаровой тензор напряжения (1.22), поэтому линейные компоненты этого вида деформаций равны между собой. В целях их определения из общего напряженного состояния выделяется шаровой тензор напряжений, от которого зависят линейные деформации от изменения объема среды.

В законе упругого изменения формы принято, что после выделения части напряжения, вызвавшей изменение объема, оставшаяся 
часть напряженного состояния, представляющая девиатор напряжения (1.19), вызывает изменение формы элемента.

Но ни та, ни другая часть деформации не доведены до логического завершения. Поскольку есть два вида деформаций по происхождению, то следует определять также два полных тензора деформаций и соответствующие им перемещения.

Правомерность выделения частей напряженного состояния, соответствующих каждому виду деформаций, вызывает сомнения по следующим причинам:

- оба вида деформаций вызваны одним и тем же напряженным состоянием, но различными его формами взаимодействия с упругой средой;

- обе части напряженного состояния в отдельности не уравновешены;

- не существует «рафинированной» объемной деформации в упругом массиве с равномерным сжатием любого элемента, она обязательно должна сопровождаться только ей принадлежащим формоизменением.

Для того чтобы внести некоторую ясность в причины возникновения рассматриваемых деформаций и классифицировать их не по форме внешнего проявления, а по происхождению, рассмотрим несколько элементарных примеров.

Пример 1. Упругое тело цилиндрической формы сжимается по вертикали равномерным давлением р без возможности бокового расширения (рис. 2.14). В механике грунтов такие испытания грунта называют компрессионными.

Тело помещается в жесткое кольцо, трение по его стенкам отсутствует. Очевидно, что деформация тела в этом случае будет протекать только за счет изменения его объема и только по вертикали, а единственная относительная деформация по вертикали в любой точке будет численно равна объемной относительной деформации:

$$
\theta=\varepsilon_{z}^{0}
$$

остальные же относительные линейные деформации будут отсутствовать, т. е. $\varepsilon_{x}^{0}=\varepsilon_{y}^{0}=0$. 


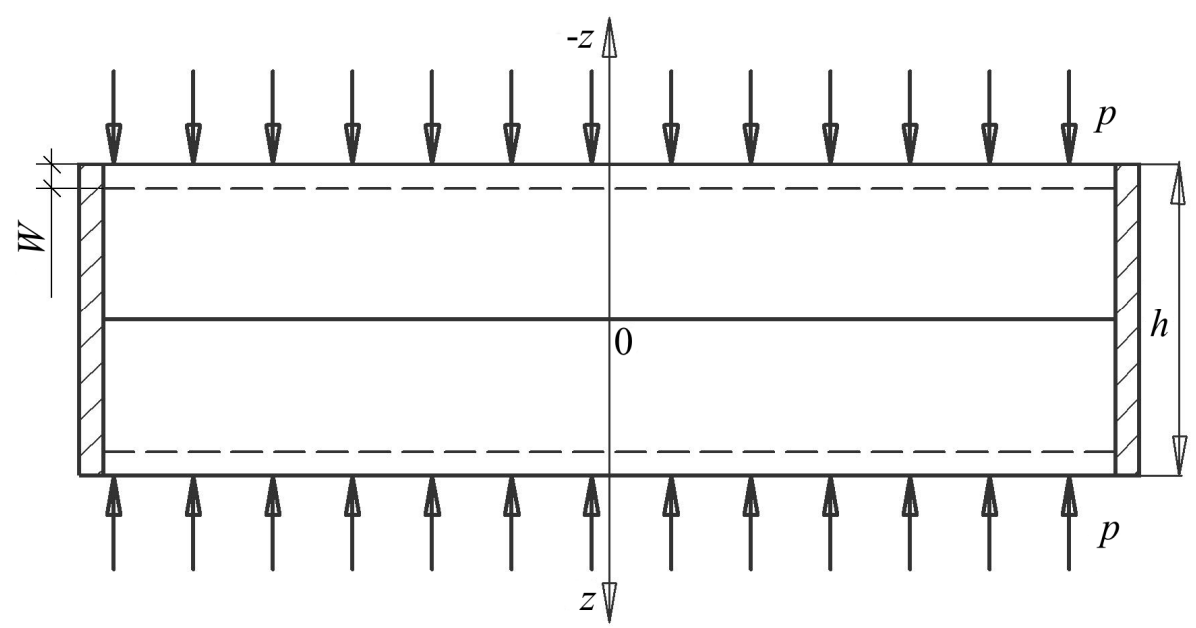

Рис. 2.14. Равномерное сжатие упругого тела по вертикали без возможности горизонтального расширения

Абсолютные перемещения торцов цилиндрического тела от объемной деформации будут наблюдаться только по вертикали:

$$
W=-0,5 \varepsilon_{z}^{0} h .
$$

Таким образом, единственная линейная относительная деформация, отличная от нуля, будет $\varepsilon_{z}^{0}$, и любой выделенный элемент изменяет свою форму, например из элементарного куба он превращается в элементарный параллелепипед, так как угловые относительные деформации отсутствуют

$$
\gamma_{x y}=\gamma_{y z}=\gamma_{z x}=0
$$

Пример 2. Упругое тело цилиндрической формы помещено без трения между двумя жесткими пластинами (см. рис. 1.3) с неизмененным расстоянием между ними и сжимается по боковой поверхности равномерно распределенной нагрузкой $p$. Это плоская деформация упругого тела. Линейные относительные деформации в этом случае также будут протекать только за счет изменения объема тела, но уже в двух направлениях - вдоль осей $x$ и $y$ (здесь принята декартова система координат, ее начало - в центре тела, ось $z$ вертикальная).

Величина объемной относительной деформации будет численно равна сумме двух равных по величине линейных относительных 
деформаций $\theta=\varepsilon_{x}^{0}+\varepsilon_{y}^{0}$, а третья линейная относительная деформация будет отсутствовать $\left(\varepsilon_{z}^{0}=0\right)$. Отсутствуют также угловые относительные деформации от изменения объема в выбранной системе координат

$$
\gamma_{x y}^{0}=\gamma_{y z}^{0}=\gamma_{z x}^{0}=0
$$

Очевидно, что объемная деформация тела в этом случае также сопровождается изменением формы: любой выделенный по направлению координат осей элемент в виде куба сжимается в двух горизонтальных направлениях и превращается в параллелепипед.

Пример 3. Упругое тело в виде шара (или любой другой объемной формы) сжимается равномерным давлением $p$ по всей поверхности (рис. 2.15).

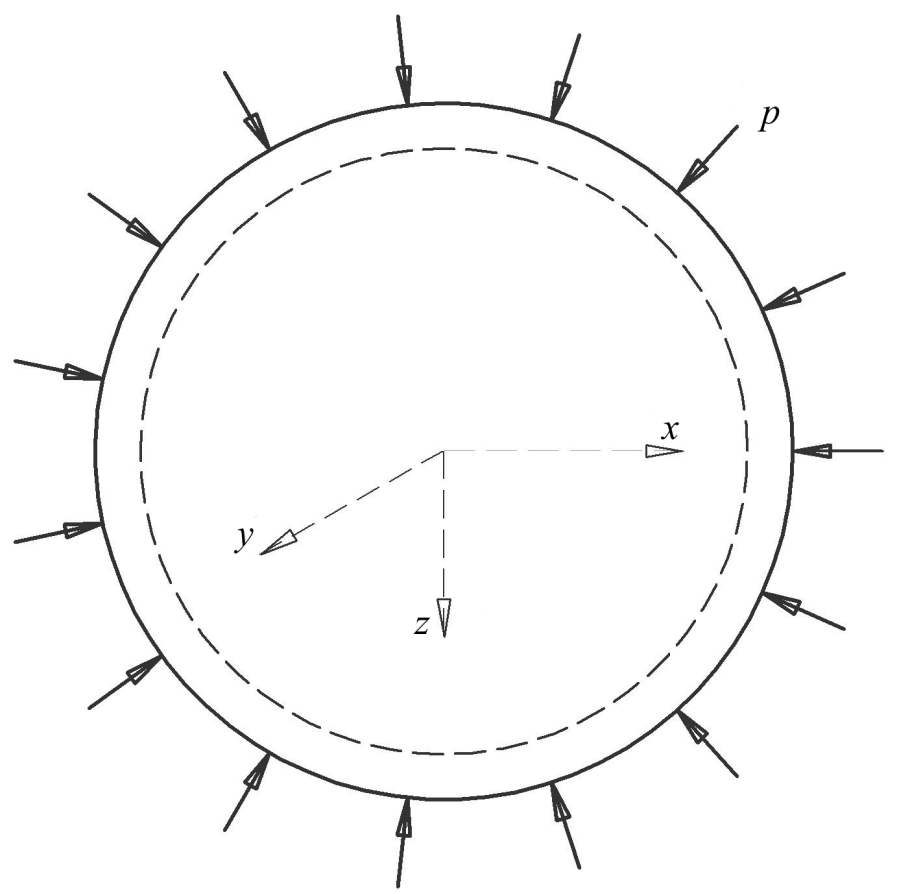

Рис. 2.15. Сжатие упругого тела всесторонним равномерным давлением

В этом случае его деформация также будет протекать только вследствие изменения объема, но, в отличие от первых двух примеров, изменения формы наблюдаться не будет. Тензор деформаций здесь будет шаровым: любой выделенный элемент будет подвержен равномерному сжатию, т. е. 


$$
\varepsilon_{x}^{0}=\varepsilon_{y}^{0}=\varepsilon_{z}^{0} \neq 0,
$$

при отсутствии угловых деформаций от изменения объема

$$
\gamma_{x y}^{0}=\gamma_{y z}^{0}=\gamma_{z x}^{0}=0,
$$

то есть относительные линейные деформации будут одинаковыми по любому направлению, а перемещения в любой точке направлены к центру.

Пример 4. Упругое тело шаровидной формы (для удобства и наглядности) подвергается сжатию равномерным давлением $p$, приложенным симметрично на двух его противоположных частях (рис. 2.16, на котором пунктиром качественно показана его форма после деформирования).

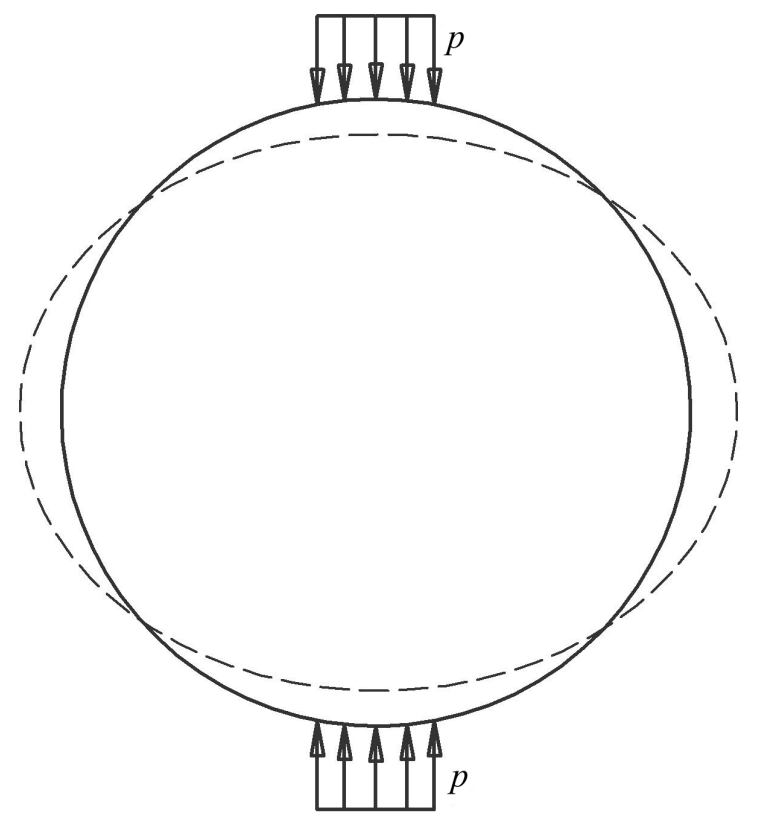

Рис. 2.16. Сжатие упругого тела нагрузкой, приложенной симметрично на двух частях его поверхности

Очевидно, что в этом случае деформации тела происходят за счет как объемного деформирования с сопутствующим ему формоизменением, так и иного формоизменения, вызванного другой причиной, независимой от объемного деформирования. Почему же такого формоизменения нет в первых трех примерах? В чем различие? А различие состоит в их напряженном состоянии. В первых трех случаях оно 
однородное, т. е. одинаковое в любой точке упругого тела, в четвертом же - различно в различных точках, т. е. в упругом теле возникают градиенты напряженного состояния, которые и вызывают дополнительное формоизменение, но это формоизменение не зависит от объемного, так как имеет другое происхождение. В дальнейшем будем называть его чистым формоизменением, или чистым сдвигом (оба термина равнозначны по существу). Отличие от общепринятого в теории упругости понятия чистого сдвига состоит в том, что в данном случае деформация чистого сдвига протекает без изменения объема, т. е сумма линейных относительных деформаций от чистого сдвига равна нулю $\varepsilon_{x}^{c}+\varepsilon_{y}^{c}+\varepsilon_{z}^{c}=0$, а напряженно-деформированное состояние упругой среды пространственное, т. е. все компоненты напряжений различные и отличаются от нуля.

Общепринятое же понятие чистого сдвига [4] относится к плоскому напряженному состоянию, когда главные напряжения

$$
\sigma_{1}=-\sigma_{2}, \quad \sigma_{3}=0
$$

и тогда по площадкам, проведенным под углом $45^{\circ}$ к главным двум $\left(\sigma_{1}\right.$ и $\left.\sigma_{2}\right)$, нормальные напряжения отсутствуют, а действуют только касательные напряжения, которые согласно обобщенному физическому закону и вызывают сдвиговые деформации.

Таким образом, понятие чистого сдвига в данной работе несколько шире этого понятия в классическом толковании, оно относится не только к плоскому напряженному состоянию, но и к пространственному.

Из рассмотренных примеров вытекают следующие важные выводы. Объемная деформация, как правило, не является всесторонне равномерной. Можно назвать единственный тривиальный случай такой деформации: равномерное всестороннее обжатие упругого тела. В общем же случае она должна всегда сопровождаться изменением формы тела. Поэтому в объемное деформирование следует отнести и вызванную им часть общего формоизменения.

Если же считать, как это принято в теории упругости, объемные деформации равномерными, то определение соответствующих перемещений не вызывает затруднений, но приводит к неприемлемым результатам. Для примера рассмотрим напряженное состояние упругого полупространства, нагруженного сосредоточенной силой $P(2.1)$ 
(2.10). Используя формулу (2.7), запишем среднее нормальное напряжение в полупространстве:

$$
\sigma_{\mathrm{cp}}=\frac{1}{3}\left(\sigma_{x}+\sigma_{y}+\sigma_{z}\right)=\frac{(1+v) P}{3 \pi} \frac{z}{R^{3}} .
$$

Учитывая (1.16), запишем линейные относительные деформации от изменения объема:

$$
\varepsilon_{x}^{0}=\varepsilon_{y}^{0}=\varepsilon_{z}^{0}=-\frac{1-2 \nu}{E} \sigma_{\mathrm{cp}}=-\frac{(1+v)(1-2 v) P}{3 \pi E} \frac{z}{R^{3}} .
$$

Поскольку мы ранее условились сжимающее напряжение обозначать со знаком плюс, то в вычислениях относительных деформаций и перемещений следует поменять знак.

Определяем перемещения вдоль оси $x$, вызванные изменениями объема упругой среды:

$$
\begin{gathered}
U^{0}=-2 \int_{0}^{x} \varepsilon_{x}^{0} d x=-\frac{-2(1+v) P z}{3 \pi E} \int_{0}^{x} \frac{d x}{\left(x^{2}+y^{2}+z^{2}\right)^{3 / 2}}= \\
=\left.\frac{2(1+v)(1-2 v) P z}{3 \pi E} \frac{1}{\left(y^{2}+z^{2}\right)} \frac{x}{\left(x^{2}+y^{2}+z^{2}\right)^{1 / 2}}\right|_{0} ^{\infty},
\end{gathered}
$$

а после подстановки пределов:

$$
U^{0}=-\frac{2 P(1+v)(1-2 v)}{3 \pi E} \frac{x z}{\left(y^{2}+z^{2}\right)\left(x^{2}+y^{2}+z^{2}\right)^{1 / 2}} .
$$

Аналогично определяются горизонтальные перемещения от изменения объема среды вдоль оси $y$. В результате имеем:

$$
V^{0}=-\frac{2 P(1+v)(1-2 v)}{3 \pi E} \frac{y z}{\left(y^{2}+z^{2}\right)\left(x^{2}+y^{2}+z^{2}\right)^{1 / 2}} .
$$


Определяем вертикальные перемещения:

$$
\begin{aligned}
W^{0}=-\int_{z}^{\infty} \varepsilon_{z}^{0} d z=-\frac{(1+v)(1-2 v) P}{3 \pi E} \int_{\infty}^{z} \frac{z d z}{\left(x^{2}+y^{2}+z^{2}\right)^{3 / 2}}= \\
=-\left.\frac{P(1+v)(1-2 v)}{3 \pi E} \frac{x}{\left(x^{2}+y^{2}+z^{2}\right)^{1 / 2}}\right|_{0} ^{\infty}
\end{aligned}
$$

а после подстановки пределов получим:

$$
W^{0}=-\frac{P(1+v)(1-2 v)}{3 \pi E} \frac{1}{\left(x^{2}+y^{2}+z^{2}\right)^{1 / 2}} .
$$

Для удобства анализа запишем перемещения (2.87)-(2.89) в цилиндрических координатах:

радиальные перемещения

$$
U_{r}^{0}=-\frac{P(1+v)(1-2 v)}{3 \pi E} \frac{r z}{r^{2}\left(r^{2}+z^{2}\right)^{1 / 2}} ;
$$

вертикальные перемещения

$$
W^{0}=\frac{P(1+v)(1-2 v)}{3 \pi E} \frac{1}{\left(r^{2}+z^{2}\right)^{1 / 2}} .
$$

Угловые деформации определяем, пользуясь зависимостями Коши (1.9). Для этого составим частные производные компонентов перемещений (2.87)-(2.89):

$$
\frac{\partial U^{0}}{\partial y}=\frac{\partial}{\partial y}\left[-A \frac{x z}{\left(y^{2}+z^{2}\right)\left(x^{2}+y^{2}+z^{2}\right)^{1 / 2}}\right],
$$




$$
\begin{aligned}
& \frac{\partial U^{0}}{\partial y}=A \frac{x y z\left[3\left(y^{2}+z^{2}\right)+2 x^{2}\right]}{\left(y^{2}+z^{2}\right)\left(x^{2}+y^{2}+z^{2}\right)^{3 / 2}} . \\
& \frac{\partial U^{0}}{\partial z}=-A \frac{x\left[\left(x^{2}+y^{2}+z^{2}\right)\left(y^{2}-z^{2}\right)-z^{2}\left(y^{2}+z^{2}\right)\right]}{\left(y^{2}+z^{2}\right)^{2}\left(x^{2}+y^{2}+z^{2}\right)^{3 / 2}} ; \\
& \frac{\partial V^{0}}{\partial x}=\frac{\partial}{\partial x}\left[-A \frac{y z}{\left(x^{2}+z^{2}\right)\left(x^{2}+y^{2}+z^{2}\right)^{1 / 2}}\right], \\
& \frac{\partial V^{0}}{\partial x}=A \frac{x y z\left[3\left(x^{2}+z^{2}\right)+2 y^{2}\right]}{\left(y^{2}+z^{2}\right)^{2}\left(x^{2}+y^{2}+z^{2}\right)^{3 / 2}} \\
& \frac{\partial V^{0}}{\partial z}=-A \frac{y\left[\left(x^{2}+y^{2}+z^{2}\right)\left(x^{2}-z^{2}\right)-z^{2}\left(x^{2}+z^{2}\right)\right]}{\left(x^{2}+z^{2}\right)^{2}\left(x^{2}+y^{2}+z^{2}\right)^{3 / 2}} ; \\
& \frac{\partial W^{0}}{\partial x}=\frac{\partial}{\partial x}\left[A \frac{1}{\left(x^{2}+y^{2}+z^{2}\right)^{1 / 2}}\right] \\
& \frac{\partial W^{0}}{\partial x}=-A \frac{x}{\left(x^{2}+y^{2}+z^{2}\right)^{3 / 2}} \\
& \frac{\partial W^{0}}{\partial y}=-A \frac{y}{\left(x^{2}+y^{2}+z^{2}\right)^{3 / 2}} .
\end{aligned}
$$

Компоненты угловых деформаций получаем в виде

$$
\gamma_{x y}^{0}=\frac{\partial U^{0}}{\partial y}+\frac{\partial V^{0}}{\partial x},
$$




$$
\begin{gathered}
\gamma_{x y}^{0}=A \frac{x y z}{\left(x^{2}+y^{2}+z^{2}\right)^{3 / 2}}\left[\frac{3\left(y^{2}+z^{2}\right)+2 x^{2}}{\left(y^{2}+z^{2}\right)^{2}}+\frac{3\left(x^{2}+z^{2}\right)+2 y^{2}}{\left(x^{2}+z^{2}\right)^{2}}\right] \\
\gamma_{y z}^{0}=\frac{\partial V^{0}}{\partial z}+\frac{\partial W^{0}}{\partial y}, \\
\gamma_{y z}^{0}=-A \frac{y\left[\left(x^{2}+y^{2}+z^{2}\right)\left(x^{2}-z^{2}\right)-\left(x^{2}+z^{2}\right)\left(x^{2}+2 z^{2}\right)\right]}{\left(x^{2}+z^{2}\right)^{2}\left(x^{2}+y^{2}+z^{2}\right)^{3 / 2}} ; \\
\gamma_{z x}^{0}=\frac{\partial W^{0}}{\partial x}+\frac{\partial U^{0}}{\partial z}, \\
\gamma_{z x}^{0}=-A \frac{x\left[\left(x^{2}+y^{2}+z^{2}\right)\left(y^{2}-z^{2}\right)-\left(y^{2}+z^{2}\right)\left(y^{2}+2 z^{2}\right)\right]}{\left(y^{2}+z^{2}\right)^{2}\left(x^{2}+y^{2}+z^{2}\right)^{3 / 2}} .
\end{gathered}
$$

В формулах (2.90)-(2.98) $A$ - постоянный коэффициент, который равен

$$
A=\frac{P(1+v)(1-2 v)}{3 \pi E} .
$$

Представленные перемещения и деформации от объемного деформирования (2.87)-(2.98), хотя формально и удовлетворяют условиям совместности (2.18), (2.19), имеют следующие недостатки, связанные с исходной предпосылкой:

1. Все угловые деформации (2.96)-(2.98) отличны от нуля, поэтому избежать формоизменения в упругой среде от объемных деформаций в предположении, что относительные линейные деформации этого вида равны между собой в каждой точке:

$$
\varepsilon_{x}^{0}=\varepsilon_{y}^{0}=\varepsilon_{z}^{0}=\frac{1}{3} \theta,
$$


невозможно, потому что они различны в различных точках полупространства, так как объемная относительная деформация является функцией координат:

$$
\theta=f(x, y, z) .
$$

Само по себе наличие угловых деформаций при отсутствии касательных напряжений - отступление от существующих физических зависимостей (1.12).

2. Принятие условия

$$
\sigma_{x}=\sigma_{y}=\sigma_{z}=\frac{1}{3}\left(\sigma_{x}+\sigma_{y}+\sigma_{z}\right)=\sigma_{\text {cp }}
$$

влечет за собой нарушение равновесия любого элемента от этой части напряжения.

Таким образом, шаровой тензор, каким представляется объемное деформирование, когда принимается

$$
\varepsilon_{x}^{0}=\varepsilon_{y}^{0}=\varepsilon_{z}^{0}
$$

и накладывается условие отсутствия формоизменения, не соответствует его истинному тензору, так как в этом случае неизбежно появление угловых деформаций, т. е. формоизменения.

Выделение частей напряженного состояния, от которых зависит объемное деформирование и формоизменение, нарушает единство напряженного состояния в целом, так как каждая из выделенных частей в отдельности не удовлетворяет условиям равновесия. При таком допущении нет оснований в отдельном рассмотрении упомянутых видов деформаций как независимых: они будут перекрещиваться, накладываться и зависеть одна от другой.

Подобное выделение объемных деформаций и деформаций формоизменения является умозрительным, оно не соответствует реальным условиям деформирования и лишено всякой логики, так как здесь потерян главный стержень, по которому следует разделять их не по внешнему признаку проявления, а по причине возникновения. Выделение каких-то частей напряженного состояния, ответственных за тот или иной вид деформации, - заблуждение. Напряженное 
состояние не отдельными своими частями, а в целом ответственно за все деформации, при этом различается только форма его влияния на упругую среду: одна форма влияния вызывает объемное деформирование, другая - чистое формоизменение.

\section{Выводы по главе 2}

Выше были проанализированы главные решения теории упругости, которые должны были стать эталонными для выполнения в них законов физики, механики, логики. К сожалению, они таковыми не могут быть из-за ряда содержащихся в них серьезных недостатков, главными из которых являются следующие.

1. В решениях теории упругости имеются разрывы сплошности среды при определении перемещений, нелогичная и противоречивая их кинематика.

2. Линейная зависимость напряженного состояния упругого массива от его деформационной характеристики $v$ (коэффициента Пуассона) приводит к тому, что перемещения в нем зависят уже от нее дважды, чем нарушается принцип линейности в линейной теории упругости.

3. Для конкретных задач с полупространством и пространством в напряженном состоянии не выполняется третий закон механики.

4. Невозможен логический предельный переход от напряженного состояния при плоской деформации полупространства к напряженному состоянию его при равномерном загружении всей поверхности.

5. Отсутствует взаимная связь между напряженно-деформированным состоянием в упругом полупространстве и пространстве от действия сосредоточенной силы.

6. Попытка разделения деформаций не по природе их возникновения, а по форме проявления принципиально не верна, так как из объемных деформаций исключается сопутствующее им формоизменение, а в деформации формоизменения собраны деформации различного происхождения - от объемного деформирования и от чистого сдвига.

Источником перечисленных и ряда других недостатков в решениях теории упругости являются принятые физические зависимости 
между напряжениями и деформациями (1.12), (1.13), названные обобщенным законом Гука, хотя он к ним не имеет отношения. Эти зависимости предложил в 1822 г. Коши [1], через 144 года после открытия Гуком закона линейной зависимости абсолютной деформации упругого тела от приложенной к нему силы (1678 г.). Зависимости (1.12), (1.13), по сути, являются феноменологическими, полученными из наблюдений деформаций тел малых размеров и механически перенесенными на упругие массивы больших размеров.

Два явления, которые наблюдаются при загружении упругого тела (объемное деформирование и чистый сдвиг), различные по природе происхождения, описываются одной формулой, устанавливающей локальную зависимость обоих видов деформаций от напряженного состояния. Из этой зависимости автоматически вытекает один из главных постулатов теории упругости о совпадении осей главных напряжений и осей главных деформаций, который стает камнем преткновения на пути принятия иных физических зависимостей. Из разрозненных элементов непросто сложить монолитный массив, так как только в массиве могут проявиться интегрирующие его свойства, а локальная зависимость не может превратиться в интегральную, как бы мы не делили массив на элементы.

Принятые физические зависимости приводят к дефектам во всех имеющихся решениях теории упругости. Кажущаяся простота и логичность этих зависимостей превратила их в догму и тем самым наложила табу на пересмотр и уточнение, поэтому исследования шли в бесперспективном направлении - получения результата путем усовершенствования и усложнения математического аппарата. А решение проблемы почти два столетия лежит на поверхности и заключается в корректировке и усовершенствовании самой модели деформирования. Такая задача и является главной целью настоящей работы. 


\section{Выбор физических зависимостей упругих тел}

\section{1. Метод аналогий в физических явлениях}

Вначале напомним некоторые важные зависимости из теории векторного поля, которые будут использованы в дальнейших изложениях.

Векторное поле градиентов связано со скалярным физическим полем зависимостью $[14 ; 15]$

$$
\bar{A}=\operatorname{grad}(u)=\frac{\partial u}{\partial x} \vec{i}+\frac{\partial u}{\partial y} \vec{j}+\frac{\partial u}{\partial z} \vec{k}
$$

где $\vec{i}, \vec{j}, \vec{k}$ - единичные векторы по направлению координатных осей соответственно $x, y, z$.

С векторным полем градиентов связано скалярное поле дивергенции:

$$
\operatorname{div} \vec{A}=\frac{\partial A_{x}}{\partial x}+\frac{\partial A_{y}}{\partial y}+\frac{\partial A_{z}}{\partial z}
$$

и векторное поле ротора

$$
\operatorname{rot} \vec{A}=\left(\frac{\partial A_{z}}{\partial y}-\frac{\partial A_{y}}{\partial z}\right) \vec{i}+\left(\frac{\partial A_{x}}{\partial z}-\frac{\partial A_{z}}{\partial x}\right) \vec{j}+\left(\frac{\partial A_{y}}{\partial x}-\frac{\partial A_{x}}{\partial y}\right) \vec{k},
$$

где $A_{x}, A_{y}, A_{z}$ - составляющие вектора $\vec{A}$ по соответствующим направлениям координатных осей. 
Дивергенция входит в одну из основных формул векторного анализа - формулу Остроградского для потока векторного поля через замкнутую поверхность (S):

$$
\oint_{(S)} \vec{A} d S=\int_{(S)} A_{n} d S=\int_{(V)} \operatorname{div} \vec{A} d V
$$

где $(S)$ - замкнутая поверхность, ограничивающая область $V$;

$A_{n}$ - проекция вектора $\vec{A}$ на направление внешней нормали к поверхности $(S)$.

Ротор входит в другую основную формулу - формулу Стокса для циркуляции вектора по замкнутой ориентированной линии $(L)$ :

$$
\int_{(L)} A_{\tau} d L=\int_{(L)} A \cos \alpha|d r|=\int_{(L)} \vec{A} d r=\operatorname{rot} \vec{A},
$$

где $A_{\tau}$ - проекция вектора $\vec{A}$ на касательную к контуру $(L)$, проведенную в направлении его обхода;

$\alpha \quad$ - угол между вектором $\vec{A}$ и касательной $\tau$;

$\vec{r} \quad$ - единичный радиус-вектор, $\vec{r}=x \vec{i}+y \vec{j}+z \vec{k}$.

Здесь и в дальнейшем речь идет об односвязной области (массиве).

Векторное поле называют потенциальным, если оно является градиентом некоторого скалярного физического поля, т. е. в случае, когда

$$
\vec{A}=\operatorname{grad}(u) .
$$

При этом функцию $u$ называют потенциалом векторного поля $\vec{A}$.

Кроме условия (3.3) к потенциальным относятся поля, у которых

$$
\operatorname{rot} \vec{A}=0
$$

либо

$$
\oint_{(L)} \vec{A} d \vec{r}=0
$$

то есть безвихревые (3.4) и бесциркуляционные (3.5) поля. 
Если поле удовлетворяет хотя бы одному из условий (3.3)-(3.5), то оно удовлетворяет и остальным условиям, т. е. условия потенциальности (3.3), отсутствия вихрей (3.4) и циркуляции (3.5) векторного поля эквивалентны. При этом потенциальная функция $u$ является гармонической:

$$
\nabla^{2} u=0
$$

где $\nabla^{2}$ - оператор Лапласа,

$$
\nabla^{2}=\frac{\partial^{2}}{\partial x^{2}}+\frac{\partial^{2}}{\partial y^{2}}+\frac{\partial^{2}}{\partial z^{2}}
$$

Известно, что многие физические явления, между которыми, казалось бы, нет ничего общего, подчиняются одним и тем же математическим зависимостям. Это положение очень четко сформулировал академик А. Н. Крылов [16, с. 28]: «...Казалось бы, что может быть общего между расчетом движения небесных светил под действием притяжения к Солнцу и между качкой корабля на волнении, или между определением так называемых вековых неравенств в движении небесных тел и крутильными колебаниями вала многоцилиндрового двигателя дизеля, работающего на корабельный винт или электрогенератор. Между тем, если написать только формулы и уравнения без слов, то нельзя отличить, какой из этих вопросов решается: уравнения одни и те же».

Совпадение уравнений не случайность, а свидетельство того факта, что все эти физические явления отражают лишь различные формы проявления движения материи. Например, стационарные процессы распространения тепла, движение электрического тока в проводящей среде, ламинарное движение идеальной жидкости, явление диффузии, распространение магнитного потока - все эти явления подчиняются одному и тому же дифференциальному уравнению в частных производных эллиптического типа, которое при отсутствии внутренних источников имеет вид

$$
\frac{\partial}{\partial x}\left[B_{1}(x, y, z) \frac{\partial u}{\partial x}\right]+\frac{\partial}{\partial y}\left[B_{2}(x, y, z) \frac{\partial u}{\partial y}\right]+\frac{\partial}{\partial z}\left[B_{3}(x, y, z) \frac{\partial u}{\partial z}\right]=0
$$


При постоянных коэффициентах $B_{i}$ зависимость (3.7) переходит в уравнение Лапласа (3.6), которому подчиняется гармоническая функция, обладающая свойствами потенциала.

В случае изучения конкретного физического явления скалярная функция и и коэффициенты $B_{i}(x, y, z)$ в уравнении (3.7) будут менять только физический смысл. Так, при движении электрического тока в проводящей среде функция $u$ будет электрическим потенциалом, а коэффициенты $B_{i}(x, y, z)$ - удельной проводимостью среды. В стационарных полях термодинамики это будут соответственно значения температуры и коэффициентов теплопроводности, в задачах фильтрации - пьезометрический напор и коэффициент фильтрации и так далее.

В табл. 3.1 сведены основные зависимости наиболее распространенных стационарных физических полей [17], описываемых уравнением (3.7). Это уравнение можно решать аналитически и экспериментально. При экспериментальном решении задач физическая природа явления играет роль только в выборе технических средств для осуществления эксперимента, точности и удобства измерений, степени соответствия между идеализированной моделью, принятой при теоретическом рассмотрении, и натурой.

Метод решения заданного уравнения путем экспериментального исследования какого-либо физического явления, описываемого этим уравнением, носит название метода математического моделирования, или метода аналогий. Кроме математического моделирования, существует также физическое моделирование, при котором сохраняется физическая природа явления. Однако математическое моделирование, которое рассматривает целый класс аналогичных явлений, описываемых одними и теми же уравнениями, является более общим, чем физическое. При моделировании уравнения эллиптического типа (3.7) наиболее удобным оказался метод электрогидродинамических аналогий (ЭГДА). Этот метод применяется в гидромеханике, электротехнике, теплотехнике, теории упругости, электронной оптике.

В 1887 г. Н. Е. Жуковский в «Лекциях по гидромеханике» [18] посвящает целую главу электрогидродинамической аналогии. Однако исключительно важная роль в развитии метода ЭГДА принадлежит академику Н. Н. Павловскому [19]. Им разработана конструкция прибора ЭДГА, его схема и методика решения задач напорной фильтрации. 


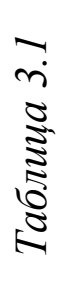

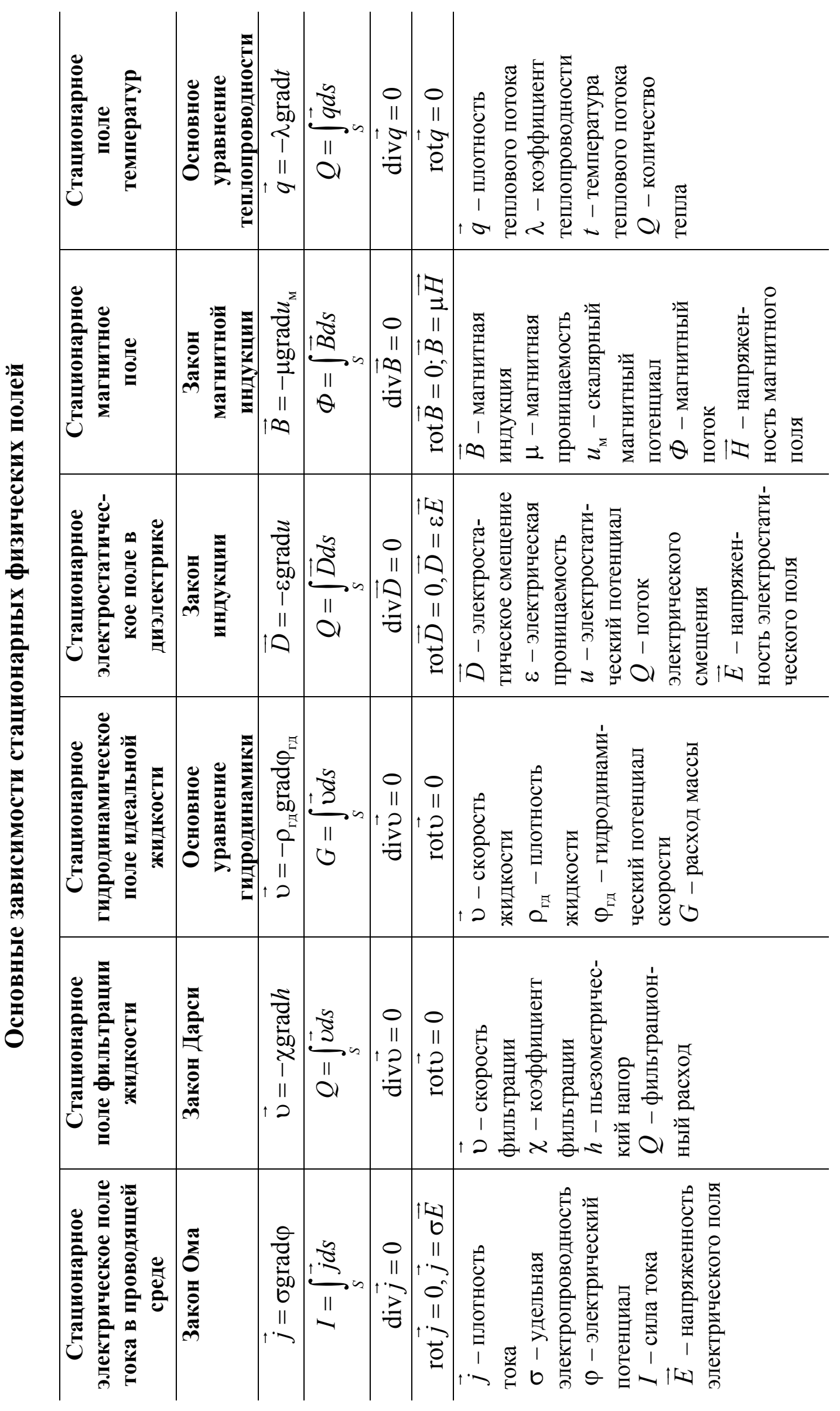


В теории упругости прибор ЭДГА впервые был применен В. А. Флориным для экспериментального определения напряженного состояния основания [8].

При анализе решений теории упругости, выполненном в предыдущей главе, обращает на себя внимание важная деталь: первый инвариант напряженного состояния упругой среды во всех задачах представляет собой скалярную гармоническую функцию, удовлетворяющую условию Лапласа

$$
\frac{\partial^{2} I_{1}}{\partial x^{2}}+\frac{\partial^{2} I_{1}}{\partial y^{2}}+\frac{\partial^{2} I_{1}}{\partial z^{2}}=0,
$$

поэтому поле такой функции может быть потенциалом векторного поля. Очевидно, что потенциальным векторным полем в упругой среде могут быть только смещения ее точек. Задача состоит в определении этих смещений.

Как уже отмечалось во второй главе, деформация любого элемента упругой среды является следствием двух наблюдаемых явлений: изменения объема среды, вызванного изменением плотности, и чистого формоизменения, которое не зависит от изменения объема. Каждому явлению должны соответствовать свои относительные и абсолютные деформации. Но поскольку оба вида деформаций всегда сопровождаются изменением формы, для отличия деформаций формоизменения, протекающих без изменения объема упругой среды, используется два равнозначных их названия: чистое формоизменение либо чистый сдвиг, и в дальнейшем, для краткости, условимся называть все абсолютные и относительные деформации от изменения объема среды объемными, а все остальные деформации, не связанные с изменением объема, - сдвиговыми. Термины «чистый сдвиг» и «чистое формоизменение» будут употребляться в отдельных случаях, требующих более четкого разграничения деформаций по их происхождению.

Логично предположить, что соответствующие двум явлениям деформации вызваны одним и тем же напряженным состоянием упругой среды, но различными формами его воздействия на среду, поэтому они должны подчиняться различным физическим закономерностям, которые не могут быть описаны одной объединенной зависимостью. В связи с этим упомянутые деформации будем определять 
раздельно, т. е. находить два полные тензоры деформаций с соответствующими им перемещениями [20-22]: тензор объемных относительных деформаций со смещениями точек среды и тензор сдвиговых относительных деформаций и смещений.

\section{2. Зависимости объемного деформирования}

Начнем с рассмотрения векторного поля смещений точек упругой среды от объемного деформирования [20; 22]. Для этого выделим в упругом массиве элемент объема $V$, ограниченный замкнутой поверхностью $S$ (рис. 3.1), и запишем изменение его объема в результате деформирования:

$$
\Delta V=\int_{(V)} \theta d V
$$

где $\theta$ - объемная относительная деформация.

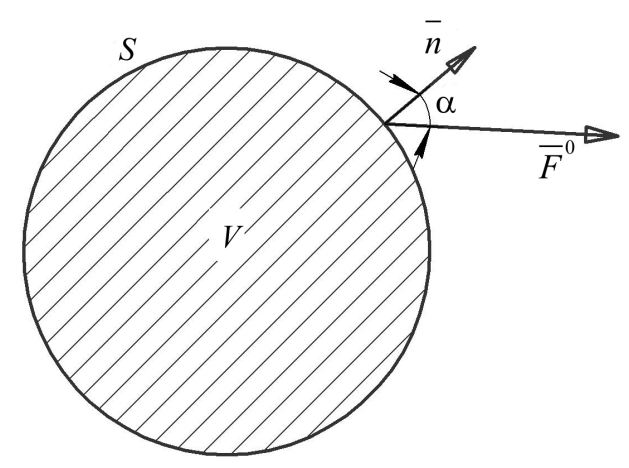

Рис. 3.1.Схема к определению изменения объема элемента упругой среды при деформировании

Изменение объема этого элемента (правая часть уравнения (3.8)) может быть определено как поверхностный интеграл произведения вектора объемного смещения любой точки выделенного объема на внешнюю нормаль к ней:

$$
\int_{(V)} \theta d V=\int_{(S)} \vec{F}^{0} \vec{n} d S,
$$


где $\vec{F}^{0}$ - вектор смещения точек поверхности $(S)$ выделенного элемента $(V)$ в результате объемного деформирования среды;

$\vec{n}$ - единичный вектор, совпадающий с внешней нормалью к поверхности $S$.

Выполнив в правой части переход от поверхностного интеграла к интегралу по всему выделенному объему на основании теоремы Остроградского, имеем:

$$
\int_{(S)} \vec{F}^{0} \vec{n} d S=\int_{(V)}\left(\frac{\partial U^{0}}{\partial x}+\frac{\partial V^{0}}{\partial y}+\frac{\partial W^{0}}{\partial z}\right) d V=\int_{(V)} \operatorname{div} \vec{F}^{0} d V .
$$

Теперь (3.9) запишем в виде

$$
\int_{(V)} \theta d V=\int_{(V)}\left(\frac{\partial U^{0}}{\partial x}+\frac{\partial V^{0}}{\partial y}+\frac{\partial W^{0}}{\partial z}\right) d V=\int_{(V)}\left(\varepsilon_{x}^{0}+\varepsilon_{y}^{0}+\varepsilon_{z}^{0}\right) d V .
$$

После приравнивания подынтегральных функций в (3.11) получим

$$
\theta=\frac{\partial U^{0}}{\partial x}+\frac{\partial V^{0}}{\partial y}+\frac{\partial W^{0}}{\partial z}=\varepsilon_{x}^{0}+\varepsilon_{y}^{0}+\varepsilon_{z}^{0}
$$

В формулах (3.10)-(3.12):

$U^{0}, V^{0}, W^{0}$ - проекции вектора смещения $\vec{F}^{0}$ в упругой среде на координатные оси, соответственно $x, y, z$ или компоненты объемного смещения;

$\varepsilon_{x}^{0}+\varepsilon_{y}^{0}+\varepsilon_{z}^{0}-$ относительные линейные деформации в упругой среде от изменения ее объема.

Выражение (3.12) является условием сплошности упругой среды при изменении ее объема.

В соответствии с существующей линейной физической зависимостью объемной относительной деформации от среднего нормального напряжения в точке (1.7), вытекающей из обобщенного физического закона и называемой законом упругого изменения объема [1], имеем: 


$$
\theta=\frac{3(1-2 v)}{E} \sigma_{\mathrm{cp}}=\frac{\sigma_{\mathrm{cp}}}{E_{0}},
$$

где

$$
\sigma_{\mathrm{cp}}=\frac{1}{3}\left(\sigma_{x}+\sigma_{y}+\sigma_{z}\right)=\frac{1}{3} I_{1}
$$

Величина

$$
\frac{E}{3(1-2 v)} \text {, }
$$

как известно, в теории упругости называется модулем объемной относительной деформации и обозначается $E_{0}$.

В дальнейшем величину среднего напряжения будем называть давлением в точке и обозначать

$$
\sigma=\sigma_{\mathrm{cp}}=\frac{1}{3}\left(\sigma_{1}+\sigma_{2}+\sigma_{3}\right)=\frac{1}{3} I_{1}
$$

а модуль объемной деформации заменим выражением

$$
E_{0}=\frac{1}{K^{0}}
$$

в котором $K^{0}$ - коэффициент объемной относительной деформации, $\mathrm{M}^{2} / \mathrm{H}$.

В результате закон изменения объема запишется в виде

$$
\theta=K^{0} \sigma
$$

Такая форма записи является более предпочтительной при описании различных физических явлений. Она позволяет сразу определить физический смысл коэффициента $K^{0}$, который представляет в данном случае объемную относительную деформацию упругой среды от единичного давления и имеет размерность, обратную напряжению. Математическая запись (3.16) может быть сформулирована так: объемная относительная деформация в любой точке упругого массива пропорциональна давлению в этой точке. 
Переход от относительной объемной деформации к соответствующим ей линейным относительным деформациям выполняется по правилу: объемная относительная деформация численно равна линейной относительной деформации, ориентированной параллельно направлению действия внешней силы:

$$
\theta=\varepsilon_{i}^{0}
$$

а остальные две линейные относительные деформации равны нулю, то есть

$$
\varepsilon_{j}^{0}=\varepsilon_{\kappa}^{0}=0
$$

Компонент перемещения $F_{i}^{0}$ также будет ориентирован параллельно линии действия внешней силы. Он определяется интегрированием относительной деформации $\varepsilon_{i}^{0}$ :

$$
F_{i}^{0}=\int \varepsilon_{i}^{0} d i
$$

а остальные два компонента перемещений при этом будут отсутствовать:

$$
F_{j}^{0}=F_{k}^{0}=0
$$

Угловые деформации определяются исходя из известных уже перемещений $F_{i}^{0}$ по зависимостям (1.9) и могут быть представлены в виде

$$
\left.\begin{array}{c}
\gamma_{i j}^{0}=\frac{\partial F_{i}^{0}}{\partial y} \\
\gamma_{j k}^{0}=0 ; \\
\gamma_{k i}^{0}=\frac{\partial F_{i}^{0}}{\partial k} .
\end{array}\right\}
$$

Углы жесткого поворота диагоналей элементарного «куба» определяются из зависимостей (1.10) и имеют следующий вид: 


$$
\left.\begin{array}{c}
2 \omega_{i}^{0}=0 ; \\
2 \omega_{j}^{0}=\frac{\partial F_{i}^{0}}{\partial k} ; \\
2 \omega_{k}^{0}=\frac{\partial F_{i}^{0}}{\partial j} .
\end{array}\right\}
$$

Таким образом, определены перемещения и полный тензор относительных деформаций от объемного деформирования, которые удовлетворяют условиям совместности (1.8). При этом, хотя величина объемной относительной деформации и соответствует закону упругого изменения объема среды (3.16), принятому в теории упругости, однако полный тензор деформаций не является шаровым.

\section{3. Зависимости чистого сдвига}

Рассмотрим векторное поле чистосдвиговых деформаций [21; 22]. С этой целью выделим элемент объема $V$ в упруго напряженном массиве вместе с векторным полем сдвиговых смещений $\vec{F}^{c}$ и составим поверхностный интеграл произведения векторов $\vec{F}^{\mathrm{c}}$ на внешнюю нормаль к поверхности $S$ выделенного элемента, который представляет приращение его объема (рис. 3.2):

$$
\Delta V=\int_{(S)}\left(\vec{F}^{\mathrm{c}} \vec{n}\right) d S=\int_{(V)}\left(\frac{\partial U^{\mathrm{c}}}{\partial x}+\frac{\partial V^{\mathrm{c}}}{\partial y}+\frac{\partial W^{\mathrm{c}}}{\partial z}\right) d V,
$$

или

$$
\Delta V=\int_{(V)} \operatorname{div} \vec{F}^{\mathrm{c}} d V
$$

Поскольку относительные и абсолютные деформации от изменения объема мы свели в одну группу и уже определили, то чистый сдвиг упругой среды должен протекать без изменения его объема $(\Delta V=0)$, поэтому 


$$
\frac{\partial U^{\mathrm{c}}}{\partial x}+\frac{\partial V^{\mathrm{c}}}{\partial y}+\frac{\partial W^{\mathrm{c}}}{\partial z}=\operatorname{div} \vec{F}^{\mathrm{c}}=0
$$

или

$$
\operatorname{div} \vec{F}^{\mathrm{c}}=\varepsilon_{x}^{\mathrm{c}}+\varepsilon_{y}^{\mathrm{c}}+\varepsilon_{z}^{\mathrm{c}}=0
$$

В формулах (3.23)-(3.25):

$F^{\mathrm{c}}, U^{\mathrm{c}}, V^{\mathrm{c}}, W^{\mathrm{c}}$ - величина сдвигового смещения любой точки среды и ее проекции на координатные оси соответственно $x, y, z$;

$\varepsilon_{x}^{\mathrm{c}}, \varepsilon_{y}^{\mathrm{c}}, \varepsilon_{z}^{\mathrm{c}}$ - относительные линейные деформации чистого сдвига в направлении соответствующих координат.

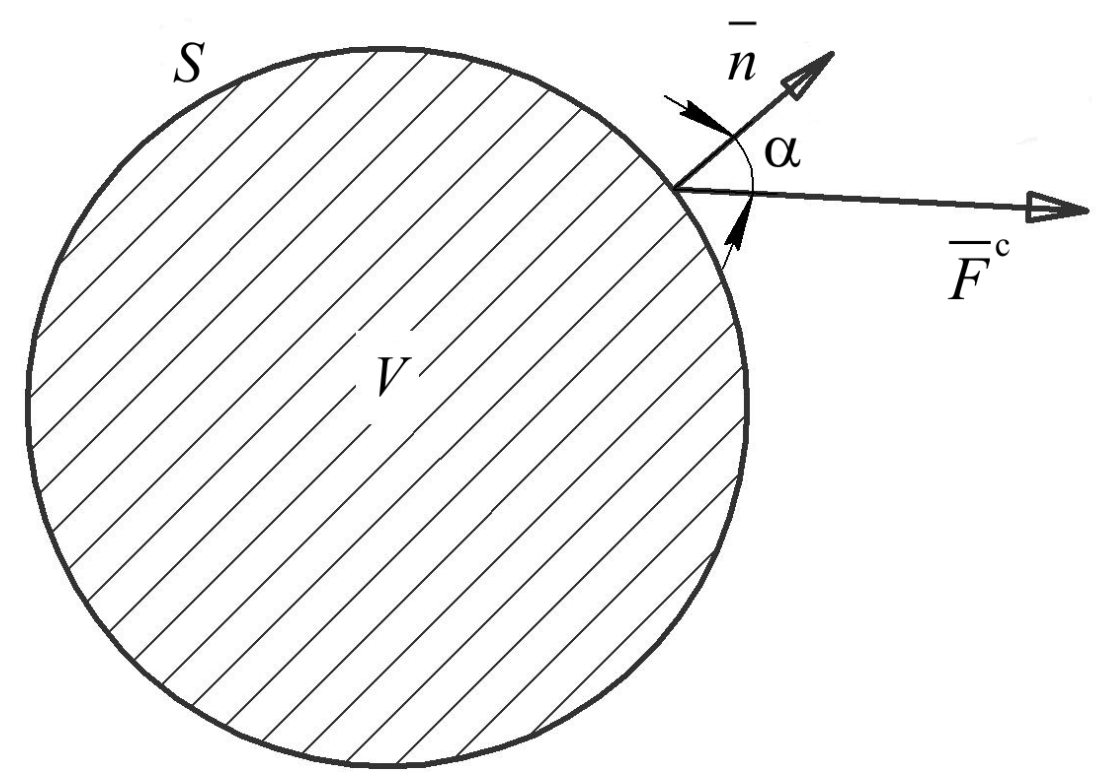

Рис. 3.2. Схема к доказательству потенциальности вектора сдвиговых смещений от чистого формоизменения при деформировании упругой среды

Условие (3.24)-(3.25) может быть выполнено лишь в том случае, когда вектор сдвиговых смещений будет иметь потенциал, т. е.

$$
\vec{F}^{\mathrm{c}}=-K^{\mathrm{c}} \operatorname{grad}(f)
$$

где $f$ - потенциал векторного поля $\vec{F}^{\text {c }}$.

Очевидно, что потенциалом векторного поля сдвиговых смещений является давление в точке (3.15), представляющее гармоническую 
функцию, поэтому сдвиговые смещения в упругом массиве будут определяться зависимостью

$$
\vec{F}^{\mathrm{c}}=-K^{\mathrm{c}} \operatorname{grad}(\sigma)
$$

а их проекции на координатные оси соответственно $x, y, z$ записываются в виде

$$
\begin{aligned}
& U^{\mathrm{c}}=-K^{\mathrm{c}} \frac{d \sigma}{d x} ; \\
& V^{\mathrm{c}}=-K^{\mathrm{c}} \frac{d \sigma}{d y} ; \\
& W^{\mathrm{c}}=-K^{\mathrm{c}} \frac{d \sigma}{d z} .
\end{aligned}
$$

В формулах (3.26)-(3.30): $K^{\mathrm{c}}$ - коэффициент сдвигового смещения, деформационная характеристика упругой среды, ее физический смысл: смещение точки упругого массива от единичного градиента давления, ${ }^{4} / \mathrm{H}$. Определение этого коэффициента не представляет никаких проблем.

Относительные деформации от чистого сдвига можно найти по зависимостям (1.8), (1.9) после подстановки в них компонентов смещений (3.28)-(3.30):

$$
\begin{gathered}
\varepsilon_{x}^{\mathrm{c}}=-K^{\mathrm{c}} \frac{\partial^{2} \sigma}{\partial x^{2}} \\
\varepsilon_{y}^{\mathrm{c}}=-K^{\mathrm{c}} \frac{\partial^{2} \sigma}{\partial y^{2}} \\
\varepsilon_{z}^{\mathrm{c}}=-K^{\mathrm{c}} \frac{\partial^{2} \sigma}{\partial z^{2}} \\
\gamma_{x y}^{\mathrm{c}}=-2 K^{\mathrm{c}} \frac{\partial^{2} \sigma}{\partial x \partial y}
\end{gathered}
$$




$$
\begin{aligned}
& \gamma_{y z}^{\mathrm{c}}=-2 K^{\mathrm{c}} \frac{\partial^{2} \sigma}{\partial y \partial z} \\
& \gamma_{z x}^{\mathrm{c}}=-2 K^{\mathrm{c}} \frac{\partial^{2} \sigma}{\partial z \partial x} .
\end{aligned}
$$

Таким образом, стационарное скалярное физическое поле давлений в упругой среде является потенциалом векторного поля смещений от чистого сдвига $\vec{F}^{c}$ (3.27). Скалярное же поле дивергенции вектора $\vec{F}^{\text {c }}$ представляет сумму линейных относительных деформаций (3.1), которая при чистом сдвиге равна нулю:

$$
\operatorname{div} \vec{F}^{\mathrm{c}}=\varepsilon_{x}^{\mathrm{c}}+\varepsilon_{y}^{\mathrm{c}}+\varepsilon_{z}^{\mathrm{c}}=0
$$

Поэтому векторное поле ротора $\vec{F}^{\text {c }}$ (3.2), представляющее жесткие повороты элементов среды, будет также отсутствовать:

$$
\begin{aligned}
& 2 \omega_{x}^{\mathrm{c}}=-K^{\mathrm{c}} \frac{\partial^{2} \sigma}{\partial y \partial z}+K^{\mathrm{c}} \frac{\partial^{2} \sigma}{\partial z \partial y}=0 ; \\
& 2 \omega_{y}^{\mathrm{c}}=-K^{\mathrm{c}} \frac{\partial^{2} \sigma}{\partial z \partial x}+K^{\mathrm{c}} \frac{\partial^{2} \sigma}{\partial x \partial z}=0 ; \\
& 2 \omega_{z}^{\mathrm{c}}=-K^{\mathrm{c}} \frac{\partial^{2} \sigma}{\partial x \partial y}+K^{\mathrm{c}} \frac{\partial^{2} \sigma}{\partial y \partial z}=0 .
\end{aligned}
$$

Условие (3.27) представляет физическую зависимость вектора сдвиговых смещений от напряженного состояния в упругой среде. В табл. 3.2 сведены основные зависимости чистого формоизменения упругой среды в физическом поле давлений. Эту таблицу можно считать продолжением табл. 3.1, в которой представлены основные зависимости различных стационарных физических полей. 
Основные зависимости

стационарного физического поля давлений в упругой среде

\begin{tabular}{|c|c|}
\hline Величина (условие) & Формула \\
\hline $\begin{array}{l}\text { Основное уравнение смещений упругой среды от } \\
\text { чистого формоизменения }\end{array}$ & $\vec{F}^{\mathrm{c}}=-K^{\mathrm{c}} \operatorname{grad}(\sigma)$ \\
\hline Приращение объема среды при деформировании & $\Delta V=\int_{(S)} \vec{F}^{0} \vec{n} d s$ \\
\hline $\begin{array}{c}\text { Условие отсутствия изменения объема элемента } \\
\text { при чистом формоизменении }\end{array}$ & $\operatorname{div} \vec{F}^{\mathrm{c}}=0$ \\
\hline $\begin{array}{l}\text { Условие отсутствия жестких поворотов элемента } \\
\text { при чистом формоизменении }\end{array}$ & $\operatorname{rot} \vec{F}^{c}=0$ \\
\hline Давление в точке & $\sigma=\frac{1}{3}\left(\sigma_{x}+\sigma_{y}+\sigma_{z}\right)$ \\
\hline
\end{tabular}

Примечания:

$\vec{F}^{\text {c }}$ - вектор смещения точки среды от чистого формоизменения;

$K^{\mathrm{c}}-$ коэффициент чистого формоизменения;

$\vec{F}^{0}$ - вектор смещения точки от изменения объема среды;

$\vec{n}$ - единичный вектор, нормальный к поверхности выделенного объема среды.

\section{4. Оценка возможности независимого существования двух видов деформаций}

Итак, согласно вышеизложенному, от стационарного поля давлений линейно зависит поле объемных относительных деформаций упругой среды $\theta=K^{0} \sigma$, которое создает векторное поле соответствующих смещений $\vec{F}^{0}$. Скалярное поле дивергенции векторного поля $\vec{F}^{0}$ отлично от нуля, так как оно представляет сумму линейных относительных деформаций от изменения объема (3.12), поэтому здесь 
не могут проявиться свойства потенциала у физического поля давлений:

$$
\operatorname{div} \vec{F}^{0}=\varepsilon_{x}^{0}+\varepsilon_{y}^{0}+\varepsilon_{z}^{0}=\theta \neq 0
$$

Одновременно это же физическое поле давлений является потенциалом сдвиговых смещений (3.27):

$$
\vec{F}^{\mathrm{c}}=-K^{\mathrm{c}} \operatorname{grad}(\sigma)
$$

Как видим, в двух видах рассмотренных деформаций проявляются два способа влияния скалярного поля давлений на формирование деформаций в упругой среде:

от величины давления линейно зависит величина объемной относительной деформации любого элемента среды (3.16);

разность давлений в различных точках среды создает его градиенты, от которых линейно зависит величина смещений чистого сдвига.

Таким образом, одно и то же скалярное физическое поле давлений проявляет себя и как обычное, действуя на среду в каждой ее точке локально, от чего в ней возникают объемные и линейные относительные деформации, и как потенциальное, действуя на среду интегрально, от чего в ней возникают смещения чистого сдвига.

Уместно здесь отметить, что тезис о возможной потенциальности перемещений в упругой среде не является новым, он изложен в учебниках по теории упругости. При этом все связывается с углами поворота диагоналей сторон элементарного куба (1.10), имеющими различные названия: жесткие повороты [1], компоненты вращения [3], элементарное вращение [2]. Суть этого тезиса в основном сводится к следующему [3].

Если в упругом теле отсутствуют углы поворотов диагоналей сторон элемента относительно осей координат, т. е. всюду в нем выполняются соотношения $2 \omega_{x}=0 ; 2 \omega_{y}=0 ; 2 \omega_{z}=0$, то рассматриваемая деформация будет потенциальной, т. е. компоненты упругих перемещений $U, V, W$ будут частными производными одной функции координат $\Phi(x, y, z)$ :

$$
U=\frac{\partial \Phi}{\partial x} ; \quad U=\frac{\partial \Phi}{\partial y} ; \quad U=\frac{\partial \Phi}{\partial z}
$$


Однако в упругом сжимаемом теле чистая деформация протекает одновременно с объемной и для ее выделения из общей деформации необходимо было внести коррективы в существующие физические зависимости, поэтому серьезного развития упомянутого тезиса не последовало.

Таким образом, разделив полную деформацию на две независимые составляющие - объемную и чистого формоизменения (или чистого сдвига) и приняв линейные зависимости объемной относительной деформации от величины давления в точке, а сдвигового смещения - от градиента этого давления, задача по определению деформаций решается без нахождения компонентов напряжений. Напряженное состояние полностью характеризует гармоническая, потенциальная функция давления, которая равна одной трети первого инварианта напряженного состояния или среднему нормальному напряжению в точке. Деформации каждого вида определяются раздельно, а полные деформации представляют алгебраическую сумму этих двух видов деформаций.

Схема решения конкретной задачи по определению напряженнодеформированного состояния упругой среды следующая:

1. По заданным граничным условиям находим гармоническую функцию давления. Граничные условия для нее могут быть заданы одним из трех способов: значениями самой функции (задача Дирихле), значениями ее производных (задача Неймана) и значениями функции на одной части границ и значениями ее производных на другой части границ (смешанная задача Дирихле-Неймана).

2. Относительная объемная деформация $\theta$ и соответствующие ей компоненты линейных деформаций $\varepsilon_{x}^{0}, \varepsilon_{y}^{0}, \varepsilon_{z}^{0}$ определяются из зависимостей (3.16)-(3.24).

3. Компоненты смещений точек среды от объемных деформаций $U^{0}, V^{0}, W^{0}$ определяются из зависимости (3.19).

4. Компоненты угловых деформаций от изменения объема $\gamma_{x y}^{0}, \gamma_{y z}^{0}, \gamma_{z x}^{0}$ находим с использованием зависимостей (1.9).

5. Углы поворотов диагоналей сторон элементарного куба от объемного деформирования $\omega_{x}^{0}, \omega_{y}^{0}, \omega_{z}^{0}$ определяются по (1.10).

6. Компоненты смещений точек среды от чистого сдвига $U^{\mathrm{c}}, V^{\mathrm{c}}, W^{\mathrm{c}}$ находятся из зависимостей (3.28)-(3.30). 
7. По зависимостям (3.14), (3.15) определяется полный тензор относительных деформаций от чистого сдвига

$$
\varepsilon_{x}^{0}, \varepsilon_{y}^{0}, \varepsilon_{z}^{0}, \gamma_{x y}^{0}, \gamma_{y z}^{0}, \gamma_{z x}^{0} .
$$

8. Полные компоненты абсолютных и относительных деформаций определяются путем алгебраического суммирования соответствующих компонентов от изменения объема и чистого сдвига:

перемещения

$$
\left.\begin{array}{l}
U=U^{0}+U^{\mathrm{c}} \\
V=V^{0}+V^{\mathrm{c}} \\
W=W^{0}+W^{\mathrm{c}}
\end{array}\right\}
$$

линейные деформации

$$
\left.\begin{array}{rl}
\varepsilon_{x} & =\varepsilon_{x}^{0}+\varepsilon_{x}^{\mathrm{c}} ; \\
\varepsilon_{y} & =\varepsilon_{y}^{0}+\varepsilon_{y}^{\mathrm{c}} ; \\
\varepsilon_{z} & =\varepsilon_{z}^{0}+\varepsilon_{z}^{\mathrm{c}}
\end{array}\right\}
$$

угловые деформации

$$
\left.\begin{array}{l}
\gamma_{x y}=\gamma_{x y}^{0}+\gamma_{x y}^{\mathrm{c}} ; \\
\gamma_{y z}=\gamma_{y z}^{0}+\gamma_{y z}^{\mathrm{c}} ; \\
\gamma_{z x}=\gamma_{z x}^{0}+\gamma_{z x}^{\mathrm{c}}
\end{array}\right\}
$$

Поскольку объемные и сдвиговые деформации независимы, то порядок их определения можно менять: после п. 2 выполнять пункты 6 , 7, а затем пункты 2-5, 8 .

Как видим, определение компонентов напряжений не является обязательным, так как деформации можно найти по функции давления, которая полностью характеризует напряженное состояние в упругом массиве. Однако для ряда задач определение компонентов напряжений может быть выполнено по зависимостям, предложенным В. А. Флориным [8], суть которых состоит в следующем. 
При любом законе распределения внешней, нормальной к плоской поверхности упругого массива нагрузки компоненты напряжений можно выразить зависимостями:

$$
\begin{aligned}
& \sigma_{x}=-z \int \frac{\partial^{2} F}{\partial x^{2}} d z+F+(1-2 v) \iint \frac{\partial^{2} F}{\partial y^{2}} d z^{2} ; \\
& \sigma_{y}=-z \int \frac{\partial^{2} F}{\partial y^{2}} d z+F+(1-2 v) \iint \frac{\partial^{2} F}{\partial x^{2}} d z^{2} ; \\
& \sigma_{z}=-z \frac{d F}{d z}+F ; \\
& \tau_{x y}=-z \int \frac{\partial^{2} F}{\partial x \partial y} d z+(1-2 v) \iint \frac{\partial^{2} F}{\partial x \partial y} d z^{2} ; \\
& \tau_{x z}=-z \frac{d F}{d x} \\
& \tau_{y z}=-z \frac{d F}{d y}
\end{aligned}
$$

где

$$
F=\frac{\sigma_{x}+\sigma_{y}+\sigma_{z}}{2(1+v)}
$$

Учитывая, что напряженное состояние в упругом массиве не зависит от коэффициента Пуассона, система уравнений (3.37) принимает вид

$$
\left.\begin{array}{cc}
\sigma_{x}=-z \int \frac{\partial^{2} \sigma}{\partial x^{2}} d z+\sigma ; & \tau_{x y}=-z \int \frac{\partial^{2} \sigma}{\partial x \partial y} d z ; \\
\sigma_{y}=-z \int \frac{\partial^{2} \sigma}{\partial y^{2}} d z+\sigma ; & \tau_{x z}=-z \frac{\partial \sigma}{\partial x} \\
\sigma_{z}=-z \frac{\partial \sigma}{\partial z}+\sigma ; & \tau_{y z}=-z \frac{\partial \sigma}{\partial y} .
\end{array}\right\}
$$

Для плоского деформирования упругого массива эта система трансформируется в 


$$
\left.\begin{array}{l}
\sigma_{x}=\sigma+z \frac{d \sigma}{d z} \\
\sigma_{z}=\sigma-z \frac{d \sigma}{d z} \\
\tau_{x z}=-z \frac{d \sigma}{d x} .
\end{array}\right\}
$$

В (3.38) и (3.39) $\sigma-$ функция давления (3.15).

\section{Выводы по главе 3}

1. Предложено разделить деформации в упругой среде на два вида по их происхождению: объемные и чистого сдвига (чистого формоизменения), определять их раздельно, а потом суммировать.

2. Для каждого вида деформаций принята своя физическая зависимость от поля давлений, представляющего собой функцию среднего нормального напряжения в упругой среде:

объемная относительная деформация линейно, через коэффициент объемной деформации, зависит от давления

$$
\theta=K^{0} \sigma
$$

вектор смещения любой точки от чистого формоизменения линейно, через коэффициент сдвигового смещения, зависит от градиента давления

$$
\vec{F}^{\mathrm{c}}=-K^{\mathrm{c}} \operatorname{grad}(\sigma)
$$

то есть является потенциальным.

3. Коэффициент объемной относительной деформации $K^{0}$ представляет обратную зависимость от известного модуля объемной деформации.

4. Коэффициент сдвигового смещения $K^{\mathrm{c}}, \mathrm{M}^{4} / \mathrm{H},-$ новая физическая деформационная константа упругой среды, представляющая 
смещение любой ее точки от единичного градиента давления, определяется экспериментально.

5. Функция давления $\sigma$ определяется аналитически или экспериментально по заданным граничным условиям, в изотропной среде она не зависит ни от какой ее физической характеристики, как и любая потенциальная функция в каком-либо физическом поле.

6. Известные величины модуля линейной деформации и коэффициента Пуассона при расчетах деформаций не нужны, но они могут быть использованы для определения коэффициента объемной относительной деформации среды.

7. Зависимость угловых деформаций от касательных напряжений не прослеживается и не используется, поэтому надобность в модуле сдвига (константа Ламе, зависящая от модуля линейной деформации и коэффициента Пуассона) отпадает.

8. Очевидным в представленных зависимостях является то, что один из двух постулатов, на которых построены соотношения Коши, о том, что главные оси деформаций совпадают с главными осями напряжений, в них не выполняется. Однако второй постулат о линейной зависимости, но не между отдельными компонентами напряжений и деформаций, а между давлением и объемной относительной деформацией, с одной стороны, и между градиентами давления и сдвиговыми смещениями, с другой стороны, сохранен в виде двух физических зависимостей соответственно (3.16) и (3.17). И в этой связи возникает следующее замечание.

Если правомерным является название физических зависимостей (1.12) или (1.13), предложенных Коши, обобщенным законом Гука, то так же правомерным может быть сохранение этого названия за предложенными линейными физическими зависимостями (3.16) и (3.27), первая из которых является локальной, а вторая интегральной от одной обобщенной характеристики напряженного состояния упругой среды - функции давления. 
ГЛАВА 4

\section{Задачи, решаемые с помошью \\ предложенных физических зависимостей}

В данной главе приводятся результаты решения ряда задач по определению напряженно-деформированного состояния однородных изотропных упругих массивов, полученные на основании применения предложенных физических зависимостей.

\section{1. Напряженно-деформированное состояние упругого изотропного полупространства при различных вариантах его нагружения}

\subsection{1. Сосредоточенная сила, приложенная по нормали к поверхности полупространства}

В некоторой точке упругого полупространства по нормали к его поверхности приложена сосредоточенная сила $P$ (рис. 4.1). Граничными условиями для потенциальной функции давления будут $[20 ; 22]:$

$$
\begin{gathered}
z=0, \quad \sigma=0 ; \\
R \rightarrow \infty, \quad \sigma \rightarrow 0,
\end{gathered}
$$

Функция должна быть четной относительно координат $x$ и $y$. При этом очевидно, что в точке приложения внешней силы $(x=y=z \rightarrow 0$ или $R \rightarrow 0)$ функция давления неограниченно возрастает $(\sigma \rightarrow \infty)$, это особенность всех задач с сосредоточенной силой. 
Условие (4.1) означает, что горизонтальные смещения поверхности от чистого сдвига должны отсутствовать, т. е. при $z=0$

$$
\frac{\partial \sigma}{\partial x}=\frac{\partial \sigma}{\partial y}=0
$$

а вектор сдвигового смещения будет направлен по нормали к поверхности.

Функцией давления, отвечающей этим условиям, является треть функции радиального напряжения (2.39), на котором мы подробно останавливались во второй главе, где эта функция приведена с учетом условий общего равновесия

$$
\sigma=\frac{1}{3} \sigma_{R}=\frac{P}{2 \pi} \frac{z}{R^{3}}
$$

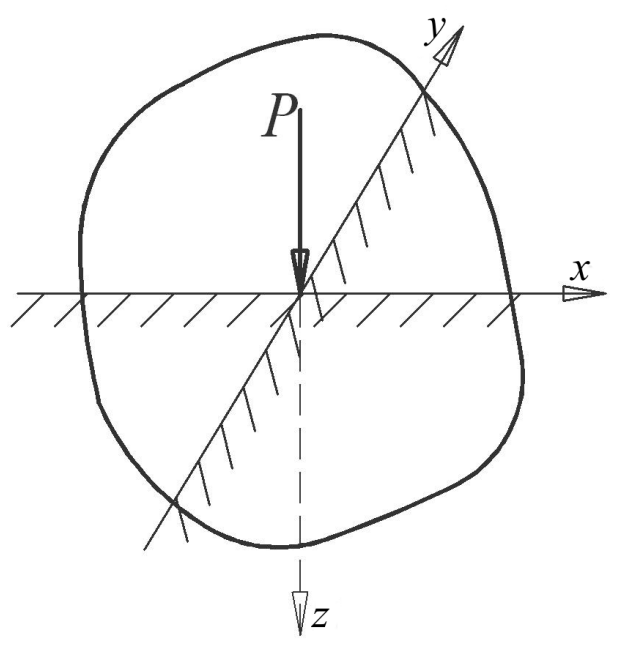

Рис. 4.1. Схема загружения полупространства сосредоточенной силой, нормальной к его поверхности

Полный тензор напряжений для данного случая нагрузки приведен в (2.40)-(2.45).

По известной функции давления определяются все перемещения и относительные деформации. Сначала находятся перемещения и относительные деформации от чистого сдвига согласно (3.28)-(3.30). 
Компонент перемещения $U^{\mathrm{c}}$

$$
\begin{gathered}
U^{\mathrm{c}}=-K^{\mathrm{c}} \frac{P}{2 \pi} \frac{\partial}{\partial x}\left[\frac{z}{\left(x^{2}+y^{2}+z^{2}\right)^{3 / 2}}\right]=K^{\mathrm{c}} \frac{3 P}{2 \pi} \frac{x z}{\left(x^{2}+y^{2}+z^{2}\right)^{5 / 2}}, \\
U^{\mathrm{c}}=K^{\mathrm{c}} \frac{3 P}{2 \pi} \frac{x z}{R^{5}}
\end{gathered}
$$

компонент перемещения $V^{\mathrm{c}}$

$$
\begin{gathered}
V^{\mathrm{c}}=-K^{\mathrm{c}} \frac{P}{2 \pi} \frac{\partial}{\partial y}\left[\frac{z}{\left(x^{2}+y^{2}+z^{2}\right)^{3 / 2}}\right]=K^{\mathrm{c}} \frac{3 P}{2 \pi} \frac{y z}{\left(x^{2}+y^{2}+z^{2}\right)^{5 / 2}}, \\
V^{\mathrm{c}}=-K^{\mathrm{c}} \frac{3 P}{2 \pi} \frac{y z}{R^{5}}
\end{gathered}
$$

компонент перемещения $W^{\mathrm{c}}$

$$
\begin{gathered}
W^{\mathrm{c}}=-K^{\mathrm{c}} \frac{P}{2 \pi} \frac{\partial}{\partial z}\left[\frac{z}{\left(x^{2}+y^{2}+z^{2}\right)^{3 / 2}}\right]=K^{\mathrm{c}} \frac{P}{2 \pi} \frac{\left(2 z^{2}-x^{2}-y^{2}\right)}{\left(x^{2}+y^{2}+z^{2}\right)^{5 / 2}}, \\
W^{\mathrm{c}}=K^{\mathrm{c}} \frac{P}{2 \pi} \frac{3 z^{2}-R^{2}}{R^{5}} .
\end{gathered}
$$

Компоненты линейных относительных деформаций от чистого сдвига в соответствии с зависимостями (3.31)-(3.33) будут:

компонент $\varepsilon_{x}^{\mathrm{c}}$ :

$$
\begin{gathered}
\varepsilon_{x}^{\mathrm{c}}=\frac{\partial U^{\mathrm{c}}}{\partial x}=K^{\mathrm{c}} \frac{3 P}{2 \pi} \frac{\partial}{\partial x}\left[\frac{x z}{\left(x^{2}+y^{2}+z^{2}\right)^{5 / 2}}\right]=K^{\mathrm{c}} \frac{3 P}{2 \pi} \frac{z\left(y^{2}+z^{2}-4 x^{2}\right)}{\left(x^{2}+y^{2}+z^{2}\right)^{7 / 2}}, \\
\varepsilon_{x}^{\mathrm{c}}=K^{\mathrm{c}} \frac{3 P}{2 \pi} \frac{z\left(R^{2}-5 x^{2}\right)}{R^{7}}
\end{gathered}
$$


компонент $\varepsilon_{y}^{c}$

$$
\begin{gathered}
\varepsilon_{y}^{\mathrm{c}}=\frac{\partial V^{\mathrm{c}}}{\partial y}=K^{\mathrm{c}} \frac{3 P}{2 \pi} \frac{\partial}{\partial y}\left[\frac{y z}{\left(x^{2}+y^{2}+z^{2}\right)^{5 / 2}}\right]=K^{\mathrm{c}} \frac{3 P}{2 \pi} \frac{z\left(x^{2}+z^{2}-4 y^{2}\right)}{\left(x^{2}+y^{2}+z^{2}\right)^{7 / 2}} \\
\varepsilon_{y}^{\mathrm{c}}=K^{\mathrm{c}} \frac{3 P}{2 \pi} \frac{z\left(R^{2}-5 y^{2}\right)}{R^{7}}
\end{gathered}
$$

компонент $\varepsilon_{z}^{c}$

$$
\begin{gathered}
\varepsilon_{z}^{\mathrm{c}}=\frac{\partial W}{\partial z}=K^{\mathrm{c}} \frac{3 P}{2 \pi} \frac{\partial}{\partial z}\left[\frac{2 z^{2}-x^{2}-y^{2}}{\left(x^{2}+y^{2}+z^{2}\right)^{5 / 2}}\right]=K^{\mathrm{c}} \frac{3 P}{2 \pi} \frac{z\left(9 x^{2}+9 y^{2}-10 z^{2}\right)}{\left(x^{2}+y^{2}+z^{2}\right)^{7 / 2}}, \\
\varepsilon_{z}^{\mathrm{c}}=K^{\mathrm{c}} \frac{P}{2 \pi} \frac{z\left(9 R^{2}-19 z^{2}\right)}{R^{7}} .
\end{gathered}
$$

Компоненты угловых относительных деформаций от чистого сдвига в соответствии с зависимостями (3.34)-(3.36) будут:

компонент $\gamma_{x y}^{\mathrm{c}}$

$$
\begin{array}{r}
\gamma_{x y}^{\mathrm{c}}=\frac{\partial U^{\mathrm{c}}}{\partial y}+\frac{\partial V^{\mathrm{c}}}{\partial x}=-2 K^{\mathrm{c}} \frac{\partial^{2} \sigma}{\partial x \partial y}=-K^{\mathrm{c}} \frac{P}{\pi} \frac{\partial^{2}}{\partial x \partial y}\left[\frac{z}{\left(x^{2}+y^{2}+z^{2}\right)^{3 / 2}}\right]= \\
=-K^{\mathrm{c}} \frac{3 P}{2 \pi} \frac{\partial^{2}}{\partial y}\left[\frac{x z}{\left(x^{2}+y^{2}+z^{2}\right)^{5 / 2}}\right],
\end{array}
$$

или

$$
\gamma_{x y}^{\mathrm{c}}=-K^{\mathrm{c}} \frac{15 P}{\pi} \frac{x y z}{R^{7}}
$$


компонент $\gamma_{y z}^{\mathrm{c}}$

$$
\begin{aligned}
\gamma_{y z}^{\mathrm{c}}=\frac{\partial V^{\mathrm{c}}}{\partial z}+\frac{\partial W^{\mathrm{c}}}{\partial y}=-2 K^{\mathrm{c}} \frac{\partial^{2} \sigma}{\partial y \partial z}=-K^{\mathrm{c}} \frac{3 P}{\pi} \frac{\partial}{\partial z}\left[\frac{y z}{\left(x^{2}+y^{2}+z^{2}\right)^{5 / 2}}\right]= \\
=K^{\mathrm{c}} \frac{3 P}{\pi} \frac{y\left(x^{2}+y^{2}-4 z^{2}\right)}{\left(x^{2}+y^{2}+z^{2}\right)^{7 / 2}}
\end{aligned}
$$

или

$$
\gamma_{y z}^{\mathrm{c}}=K^{\mathrm{c}} \frac{3 P}{\pi} \frac{y\left(R^{2}-5 z^{2}\right)}{R^{7}}
$$

компонент $\gamma_{z x}^{\mathrm{c}}$

$$
\begin{gathered}
\gamma_{z x}^{\mathrm{c}}=\frac{\partial W^{\mathrm{c}}}{\partial x}+\frac{\partial U^{\mathrm{c}}}{\partial z}=-2 K^{\mathrm{c}} \frac{\partial^{2} \sigma}{\partial y \partial z}=-K^{\mathrm{c}} \frac{3 P}{\pi} \frac{\partial}{\partial z}\left[\frac{x z}{\left(x^{2}+y^{2}+z^{2}\right)^{5 / 2}}\right] \\
\gamma_{z x}^{\mathrm{c}}=K^{\mathrm{c}} \frac{3 P}{\pi} \frac{x\left(x^{2}+y^{2}-4 z^{2}\right)}{\left(x^{2}+y^{2}+z^{2}\right)^{7 / 2}}
\end{gathered}
$$

или

$$
\gamma_{z x}^{\mathrm{c}}=K^{\mathrm{c}} \frac{3 P}{\pi} \frac{x\left(R^{2}-5 z^{2}\right)}{R^{7}} .
$$

Очевидно, что углы поворотов диагоналей элементарного куба (жесткие повороты) от чистого сдвига будут отсутствовать:

$$
\omega_{x}^{\mathrm{c}}=\omega_{y}^{\mathrm{c}}=\omega_{z}^{\mathrm{c}}=0 .
$$

Таким образом, найдены абсолютные и относительные деформации от чистого сдвига. 
Переходим теперь к определению объемных деформаций.

Объемная относительная деформация в соответствии с (3.26) будет

$$
\theta=K^{0} \sigma=K^{0} \frac{P}{2 \pi} \frac{z}{R^{3}}
$$

Линейная относительная деформация от изменения объема в направлении, параллельном действию приложенной силы, численно равна объемной относительной деформации (3.17)-(3.20), т. е.

$$
\varepsilon_{z}^{0}=\theta=K^{0} \frac{P}{2 \pi} \frac{z}{R^{3}}
$$

а остальные линейные относительные деформации отсутствуют

$$
\varepsilon_{x}^{0}=\varepsilon_{y}^{0}=0 .
$$

По известной линейной относительной деформации $\varepsilon_{z}^{0}$ определяются вертикальные перемещения точек среды от изменения объема

$$
W^{0}=\int_{\infty}^{z} \varepsilon_{z}^{0} d z=\int_{\infty}^{z} K^{0} \frac{P z d z}{2 \pi\left(x^{2}+y^{2}+z^{2}\right)^{3 / 2}} .
$$

Согласно [23] этот интеграл равен:

$$
W^{0}=\left.K^{0} \frac{P}{2 \pi} \frac{1}{\left(x^{2}+y^{2}+z^{2}\right)^{1 / 2}}\right|_{\infty} ^{z},
$$

а после подстановки пределов получим

$$
W^{0}=\frac{K^{0} P}{2 \pi} \frac{1}{\left(x^{2}+y^{2}+z^{2}\right)^{1 / 2}}=K^{0} \frac{P}{2 \pi R} .
$$

Компоненты угловых деформаций от изменения объема согласно (1.9) будут: 


$$
\begin{gathered}
\gamma_{x y}^{0}=\frac{\partial U^{0}}{\partial y}+\frac{\partial V^{0}}{\partial x}=0+0 \\
\gamma_{y z}^{0}=\frac{\partial V^{0}}{\partial z}+\frac{\partial W^{0}}{\partial y}=0+K^{0} \frac{P}{2 \pi} \frac{\partial}{\partial y}\left[\frac{1}{\left(x^{2}+y^{2}+z^{2}\right)^{1 / 2}}\right], \\
\gamma_{y z}^{0}=-K^{0} \frac{P}{2 \pi} \frac{y}{\left(x^{2}+y^{2}+z^{2}\right)^{3 / 2}},
\end{gathered}
$$

или

$$
\begin{aligned}
\gamma_{y z}^{0}=-K^{0} \frac{P}{2 \pi} \frac{y}{R^{3}} & \\
\gamma_{z x}^{0}=\frac{\partial W^{0}}{\partial x}+\frac{\partial U^{0}}{\partial z}=K^{0} \frac{P}{2 \pi} \frac{\partial}{\partial x}\left[\frac{1}{\left(x^{2}+y^{2}+z^{2}\right)^{1 / 2}}\right] & +0= \\
& =-K^{0} \frac{P}{2 \pi} \frac{x}{\left(x^{2}+y^{2}+z^{2}\right)^{3 / 2}},
\end{aligned}
$$

или

$$
\gamma_{z x}^{0}=-K^{0} \frac{P}{2 \pi} \frac{x}{R^{3}} .
$$

Углы поворотов диагоналей сторон элементарного куба от объемных деформаций будут отличными от нуля, они определяются из зависимостей (1.10):

$$
\omega_{x}^{0}=\frac{\partial V^{0}}{\partial z}-\frac{\partial W^{0}}{\partial y}=0-K^{0} \frac{P}{2 \pi} \frac{\partial}{\partial y}\left[\frac{1}{\left(x^{2}+y^{2}+z^{2}\right)^{\frac{1}{2}}}\right]=K^{0} \frac{P}{2 \pi} \frac{y}{\left(x^{2}+y^{2}+z^{2}\right)^{\frac{3}{2}}},
$$

или

$$
\omega_{x}^{0}=K^{0} \frac{P}{2 \pi} \frac{y}{R^{3}} ;
$$




$$
\begin{aligned}
\omega_{y}^{0}=\frac{\partial W^{0}}{\partial x}-\frac{\partial U^{0}}{\partial z}=K^{0} \frac{P}{2 \pi} \frac{\partial}{\partial x}\left[\frac{1}{\left(x^{2}+y^{2}+z^{2}\right)^{1 / 2}}\right] & -0= \\
& =K^{0} \frac{P}{2 \pi} \frac{x}{\left(x^{2}+y^{2}+z^{2}\right)^{3 / 2}},
\end{aligned}
$$

или

$$
\omega_{y}^{0}=K^{0} \frac{P}{2 \pi} \frac{x}{R^{3}} .
$$

Жесткий поворот элемента относительно оси $z$ будет отсутствовать:

$$
\omega_{z}^{0}=\frac{\partial U^{0}}{\partial y}-\frac{\partial V^{0}}{\partial x}=0-0 .
$$

Полные компоненты перемещений и деформаций в данной задаче представляют алгебраическую сумму соответствующих компонентов от чистого сдвига и объемного деформирования.

\subsection{2. Сосредоточенная сила, приложенная по касательной к поверхности полупространства}

Соответствующая схема приложения силы показана на рис. 4.2.

Граничными условиями для функции давления в этом случае являются:

1) $z=0, \frac{\partial \sigma}{\partial z}=0$, (т. е. отсутствие вертикальных перемещений поверхности от чистого сдвига);

2) $R \rightarrow \infty, \sigma \rightarrow 0$;

3) функция должна быть четной относительно координаты $y$ и нечетной относительно координаты $x$;

4) функция должна обеспечить радиальность напряженного состояния в полупространстве. 
Функцией давления, отвечающей этим условиям, является гармоническая функция

$$
\sigma=\frac{1}{3} A \frac{x}{\left(x^{2}+y^{2}+z^{2}\right)^{3 / 2}}=\frac{1}{3} A \frac{x}{R^{3}} .
$$

Постоянный коэффициент $A$ определяется из условия общего равновесия. С этой целью параллельно координатной плоскости zоу на расстояниях $\pm x$ от нее проведем две плоскости (рис. 4.3).

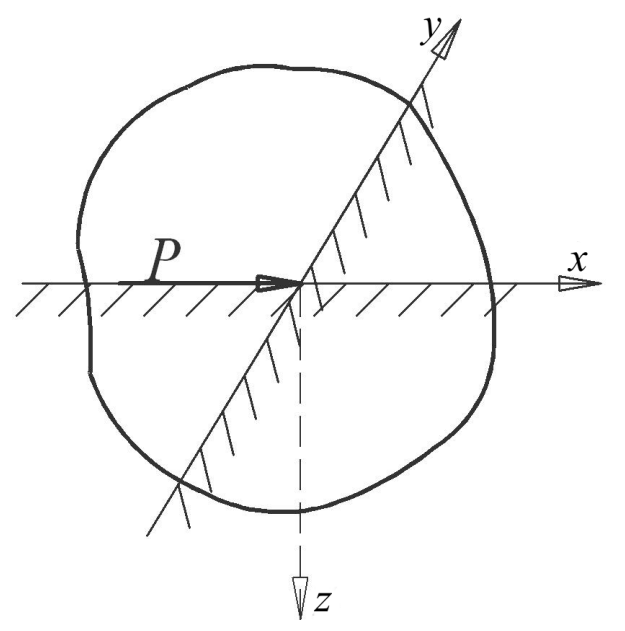

Рис. 4.2. Схема загружения полупространства касательной к поверхности сосредоточенной силой

Очевидно, что в левой плоскости на расстоянии $-x$ от координатной плоскости zоу среда испытывает нормальные растягивающие напряжения. При этом независимо от расстояний $\pm x$ слева и справа суммарное реактивное давление в каждой плоскости должно быть равным половине приложенной силы $P$, так как левая и правая части полупространства вместе уравновешивают внешнюю силу. Поскольку распределение напряжений является радиальным, то компонент нормального напряжения $\sigma_{x}$ описывается зависимостью

$$
\begin{gathered}
\sigma_{x}=\sigma_{R} \frac{x}{R} \frac{x}{R}=\sigma_{R} \frac{x^{2}}{R^{2}}=A \frac{x}{R^{3}} \frac{x^{2}}{R^{2}}, \\
\sigma_{x}=A \frac{x^{3}}{R^{5}} .
\end{gathered}
$$




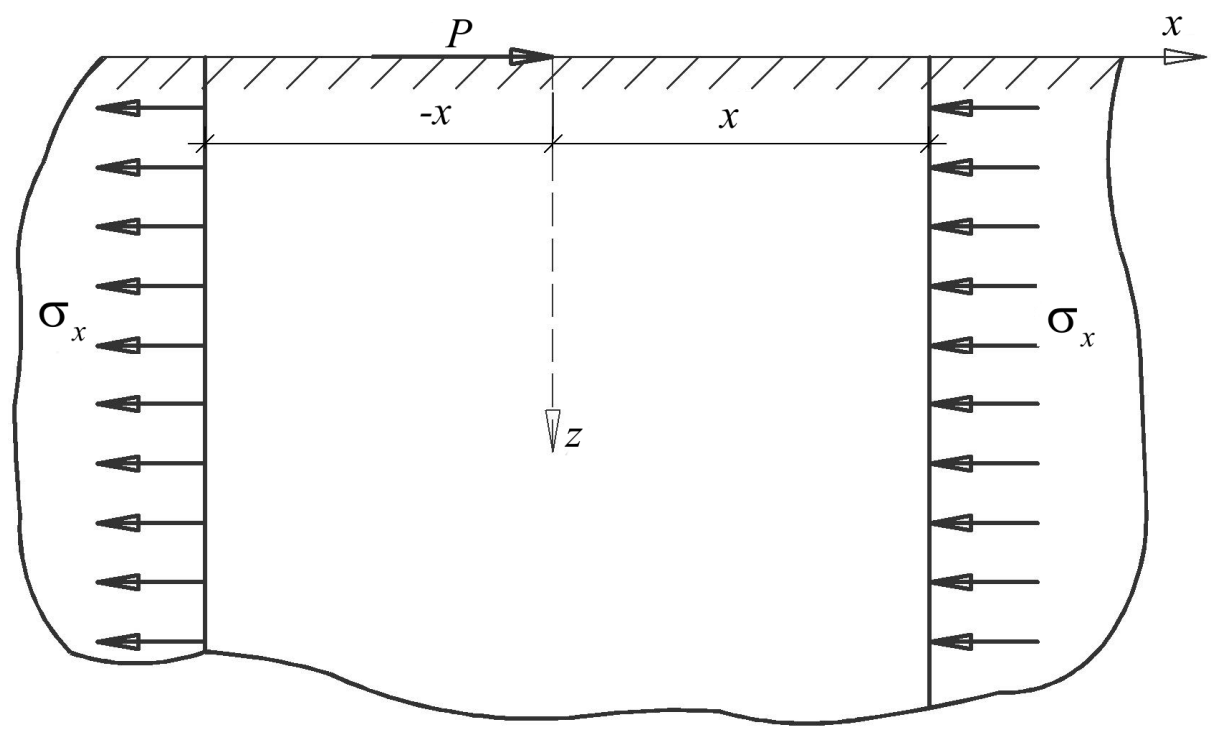

Рис. 4.3. Схема интегрирования компонента $\sigma_{x}$ для определения коэффициента $A$ в (4.20)

Составим интеграл для определения суммарного реактивного давления по одной из плоскостей:

$$
\begin{aligned}
& \int_{y=-\infty}^{y=\infty} \int_{z=0}^{z=\infty} \sigma_{x} d y d z=A x^{3} \int_{y=-\infty}^{y=\infty} \int_{z=0}^{z=\infty} \frac{d y d z}{\left(x^{2}+y^{2}+z^{2}\right)^{5 / 2}}= \\
& =\left.A x^{3} \frac{2^{2}}{3} \int_{y=-\infty}^{y=+\infty} \frac{1}{\left(x^{2}+y^{2}\right)^{2}}\left[\frac{z}{\left(x^{2}+y^{2}+z^{2}\right)^{1 / 2}}-\frac{1}{3} \frac{z^{3}}{\left(x^{2}+y^{2}+z^{2}\right)^{3 / 2}}\right]\right|_{z=0} ^{z=\infty} d y= \\
& =A x^{3} \frac{2}{3} \int_{y=-\infty}^{y=+\infty} \frac{d y}{\left(x^{2}+y^{2}\right)^{2}}=\frac{2 A x^{3}}{3}\left|\frac{y}{2 x^{2}\left(x^{2}+y^{2}\right)}+\frac{1}{2 z^{3}} \operatorname{arctg} \frac{y}{x}\right|_{y=-\infty}^{y=+\infty}= \\
& =\frac{2 A x^{3}}{3} \frac{1}{2 x^{3}} \pi=\frac{A \pi}{3} .
\end{aligned}
$$

Эта величина должна быть равной половине внешней силы

$$
A \frac{\pi}{3}=\frac{P}{2}
$$


откуда

$$
A=\frac{3 P}{2 \pi},
$$

что совпадает с (2.38) и подтверждает взаимосвязь решений задач о напряженно-деформированном состоянии в полупространстве.

Компоненты напряжений в данной задаче, учитывая радиальное распределение полного напряжения, определяются по известной функции первого инварианта напряженного состояния

$$
I_{1}=\sigma_{R}=3 \sigma=\frac{3 P}{2 \pi} \frac{x}{R^{3}}
$$

из зависимостей (2.29)-(2.37), в результате чего получим:

$$
\begin{aligned}
& \sigma_{x}=\frac{3 P}{2 \pi} \frac{x^{3}}{R^{5}} \\
& \sigma_{y}=\frac{3 P}{2 \pi} \frac{x y^{2}}{R^{5}} \\
& \sigma_{z}=\frac{3 P}{2 \pi} \frac{x z^{2}}{R^{5}} \\
& \tau_{x y}=\frac{3 P}{2 \pi} \frac{x^{2} y}{R^{5}} \\
& \tau_{y z}=\frac{3 P}{2 \pi} \frac{x y z}{R^{5}} \\
& \tau_{z x}=\frac{3 P}{2 \pi} \frac{x^{2} z}{R^{5}}
\end{aligned}
$$

Компоненты перемещений от чистого сдвига находим по зависимостям (3.28)-(3.30): 


$$
U^{\mathrm{c}}=-K^{\mathrm{c}} \frac{P}{2 \pi} \frac{\partial}{\partial x}\left[\frac{x}{\left(x^{2}+y^{2}+z^{2}\right)^{3 / 2}}\right]=-K^{\mathrm{c}} \frac{P}{2 \pi} \frac{y^{2}+z^{2}-2 x^{2}}{\left(x^{2}+y^{2}+z^{2}\right)^{5 / 2}},
$$

или

$$
\begin{gathered}
U^{\mathrm{c}}=K^{\mathrm{c}} \frac{P}{2 \pi} \frac{3 x^{2}-R^{2}}{R^{5}} \\
V^{\mathrm{c}}=-K^{\mathrm{c}} \frac{P}{2 \pi} \frac{\partial}{\partial y}\left[\frac{x}{\left(x^{2}+y^{2}+z^{2}\right)^{3 / 2}}\right]=K^{\mathrm{c}} \frac{3 P}{2 \pi} \frac{x y}{\left(x^{2}+y^{2}+z^{2}\right)^{5 / 2}},
\end{gathered}
$$

или

$$
\begin{gathered}
V^{\mathrm{c}}=-K^{\mathrm{c}} \frac{3 P}{2 \pi} \frac{x y}{R^{5}} \\
W^{\mathrm{c}}=-K^{\mathrm{c}} \frac{P}{2 \pi} \frac{\partial}{\partial z}\left[\frac{x}{\left(x^{2}+y^{2}+z^{2}\right)^{3 / 2}}\right]=K^{\mathrm{c}} \frac{P}{2 \pi} \frac{x z}{\left(x^{2}+y^{2}+z^{2}\right)^{5 / 2}},
\end{gathered}
$$

или

$$
W^{\mathrm{c}}=K^{\mathrm{c}} \frac{3 P}{2 \pi} \frac{x z}{R^{5}} .
$$

Компоненты линейных относительных деформаций от объемного деформирования определяются с использованием зависимостей (3.17)-(3.20):

$$
\begin{gathered}
\varepsilon_{x}^{0}=K^{0} \frac{P}{2 \pi} \frac{x}{R^{3}} ; \\
\varepsilon_{y}^{0}=\varepsilon_{z}^{0}=0 .
\end{gathered}
$$

Перемещения точек полупространства от объемного деформирования в направлении оси $x$ определяются интегрированием: 


$$
\begin{gathered}
U^{0}=\int_{K}^{\infty} \varepsilon_{x}^{0} d x=K^{0} \frac{P}{2 \pi} \int_{x}^{\infty} \frac{x d x}{\left(x^{2}+y^{2}+z^{2}\right)^{3 / 2}}=K^{0} \frac{P}{2 \pi}\left|-\frac{1}{\left(x^{2}+y^{2}+z^{2}\right)^{1 / 2}}\right|_{x}^{\infty}, \\
U^{0}=K^{0} \frac{P}{2 \pi} \frac{1}{R} .
\end{gathered}
$$

Определение угловых относительных деформаций от сдвигового и объемного деформирования по известным уже перемещениям не вызывает затруднений, поэтому они здесь не приведены.

Компоненты углов поворота элементов от сдвигового деформирования будут отсутствовать, как и во всех задачах, а соответствующие компоненты от объемного деформирования по известному вектору перемещений $U^{0}$ определяются из зависимостей (1.10) аналогично тому, как это было выполнено для сосредоточенной силы $P$, направленной по нормали к поверхности полупространства. Поскольку эта операция не вызывает затруднений, то ее результаты здесь не приведены.

\subsection{3. Сосредоточенная сила, приложенная наклонно к поверхности полупространства}

В том случае, когда сила действует наклонно к поверхности полупространства, составляя углы наклона $\alpha, \beta$ и $\gamma$ к координатным осям соответственно $x, y, z$ (рис. 4.4), порядок решения задачи будет следующий.

1. Действующая сила раскладывается на три составляющие, направленные по координатным осям:

касательная составляющая по направлению оси $x P_{x}=P \cos \alpha$;

касательная составляющая по направлению оси $y P_{y}=P \cos \beta$;

нормальная составляющая по направлению оси $z P_{z}=P \cos \gamma$.

2. Определяются все компоненты напряженно-деформированного состояния в отдельности от каждой силы.

3. Полные компоненты напряженно-деформированного состояния от действующей силы $P$ представляются алгебраической суммой соответствующих компонентов от сил $P_{x}, P_{y}$ и $P_{z}$. 
В целях более наглядного представления решения данной задачи запишем сначала решение для случая, когда внешняя сила приложена по касательной к поверхности и направлена вдоль оси $y$.

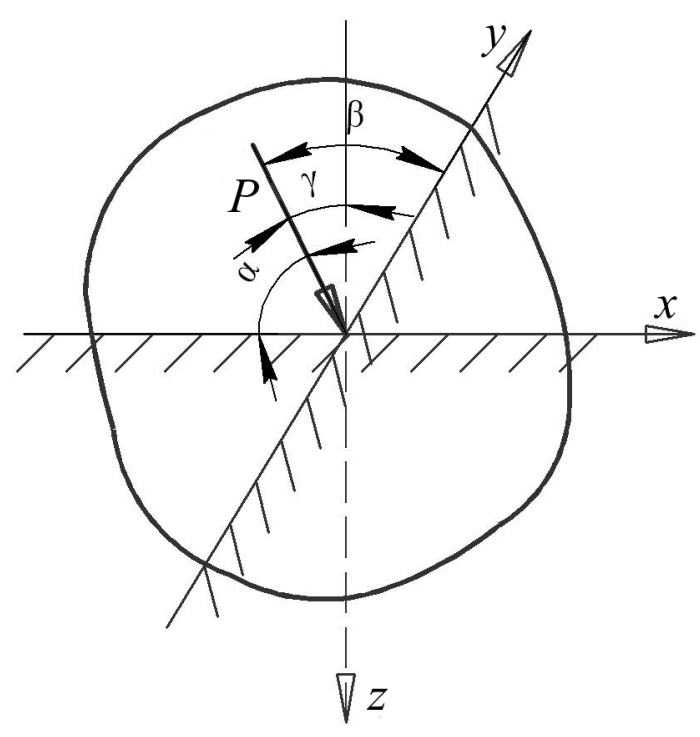

Рис. 4.4. Схема загружения поверхности полупространства наклонной сосредоточенной силой

Функция давления в этом случае будет

$$
\sigma=\frac{P}{2 \pi} \frac{y}{\left(x^{2}+y^{2}+z^{2}\right)^{3 / 2}} ;
$$

компоненты напряжений

$$
\begin{aligned}
& \sigma_{x}=\frac{3 P}{2 \pi} \frac{x^{2} y}{R^{5}} ; \quad \tau_{x y}=\frac{3 P}{2 \pi} \frac{x y^{2}}{R^{5}} ; \\
& \sigma_{y}=\frac{3 P}{2 \pi} \frac{y^{3}}{R^{5}} ; \quad \tau_{y z}=\frac{3 P}{2 \pi} \frac{y^{2} z}{R^{5}} ; \\
& \sigma_{z}=\frac{3 P}{2 \pi} \frac{y z^{2}}{R^{5}} ; \quad \tau_{z x}=\frac{3 P}{2 \pi} \frac{x y z}{R^{5}} ;
\end{aligned}
$$


компоненты перемещений от чистого сдвига

$$
\begin{gathered}
U^{\mathrm{c}}=K^{\mathrm{c}} \frac{3 P}{2 \pi} \frac{x y}{R^{5}} ; \\
V^{\mathrm{c}}=K^{\mathrm{c}} \frac{P}{2 \pi} \frac{\left(3 y^{2}-R^{2}\right)}{R^{5}} ; \\
W^{\mathrm{c}}=K^{\mathrm{c}} \frac{3 P}{2 \pi} \frac{y z}{R^{5}} ;
\end{gathered}
$$

перемещения от объемного деформирования

$$
\begin{gathered}
V^{0}=K^{0} \frac{P}{2 \pi} \frac{1}{R} ; \\
U^{0}=W^{0}=0 .
\end{gathered}
$$

Теперь запишем полное решение задачи для случая, когда внешняя сила действует наклонно к поверхности полупространства, которое представляет сумму решений от действия трех составляющих силы $P$, приложенных в одной точке $P_{x}, P_{y}, P_{z}$ :

функция давления

$$
\sigma=\frac{P}{2 \pi R^{3}}(x \cos \alpha+y \cos \beta+\cos \gamma)
$$

компоненты напряжений:

$$
\begin{aligned}
& \sigma_{x}=\frac{3 P}{2 \pi} \frac{x^{2}}{R^{5}}(x \cos \alpha+y \cos \beta+z \cos \gamma) ; \\
& \sigma_{y}=\frac{3 P}{2 \pi} \frac{y^{2}}{R^{5}}(x \cos \alpha+y \cos \beta+z \cos \gamma) ; \\
& \sigma_{z}=\frac{3 P}{2 \pi} \frac{z^{2}}{R^{5}}(x \cos \alpha+y \cos \beta+z \cos \gamma) ;
\end{aligned}
$$




$$
\begin{aligned}
\tau_{x y} & =\frac{3 P}{2 \pi} \frac{x y}{R^{5}}(x \cos \alpha+y \cos \beta+z \cos \gamma) ; \\
\tau_{y z} & =\frac{3 P}{2 \pi} \frac{y z}{R^{5}}(x \cos \alpha+y \cos \beta+z \cos \gamma) ; \\
\tau_{z x} & =\frac{3 P}{2 \pi} \frac{x z}{R^{5}}(x \cos \alpha+y \cos \beta+z \cos \gamma) ;
\end{aligned}
$$

компоненты сдвиговых смещений:

$$
\begin{aligned}
& U^{\mathrm{c}}=K^{\mathrm{c}} \frac{P}{2 \pi R^{5}}\left[\left(3 x^{2}-R^{2}\right) \cos \alpha+3 x y \cos \beta+3 x z \cos \gamma\right] ; \\
& V^{\mathrm{c}}=K^{\mathrm{c}} \frac{P}{2 \pi R^{5}}\left[3 x y \cos \alpha+\left(3 y^{2}-R^{2}\right) \cos \beta+3 y z \cos \gamma\right] ; \\
& W^{\mathrm{c}}=K^{\mathrm{c}} \frac{P}{2 \pi R^{5}}\left[3 x z \cos \alpha+3 y z \cos \beta+\left(3 z^{2}-R^{2}\right) \cos \gamma\right] ;
\end{aligned}
$$

компоненты перемещений от объемного деформирования

$$
\begin{aligned}
& U^{0}=K^{0} \frac{P \cos \alpha}{2 \pi R} ; \\
& V^{0}=K^{0} \frac{P \cos \beta}{2 \pi R} ; \\
& W^{0}=K^{0} \frac{P \cos \gamma}{2 \pi R} .
\end{aligned}
$$

Линейные и угловые относительные деформации несложно получить из зависимостей соответственно (3.31)-(3.33) и (3.34)-(3.36), используя полученные сдвиговые смещения (4.41)-(4.43) и объемные смещения (4.44)-(4.46). Полученное решение (4.34)-(4.46) можно несколько упростить, если есть возможность выбора ориентации координатной системы. Выбор следует осуществить таким образом, чтобы ее начало совпадало с точкой приложения внешней силы, а сам 
вектор внешней силы находился в одной из вертикальных координатных плоскостей. Тогда одна из составляющих внешней силы ( $P_{x}$ либо $P_{y}$ ) превращается в нуль. Например, если действующая сила лежит в плоскости xoz, то в формулах (4.34)-(4.46) исчезают слагаемые, содержащие множитель $\cos \beta$, и наоборот, если действующая сила находится в плоскости $x o y$, то исчезают слагаемые, содержащие $\cos \alpha$. В том же случае, когда внешняя сила приложена по нормали к плоскости, ограничивающей полупространство, мы получим приведенное выше решение (4.4)-(4.21) как частный случай решения (4.34)-(4.46).

\subsection{4. Нормальная нагрузка, равномерно распределенная вдоль линий конечной и бесконечной длины}

По нормали к поверхности полупространства вдоль некоторой линии равномерно распределена погонная нагрузка интенсивностью $P$, H/м [24].

Напряженное состояние в этой задаче можно получить путем интегрирования решения для сосредоточенной силы (2.39)-(2.45) по одной из координат на поверхности полупространства в соответствии с рис. 4.5 .

Функция давления для этого случая определяется интегрированием (2.39):

$$
\begin{gathered}
\sigma=-\frac{P z}{2 \pi} \int_{-b}^{b} \frac{d(y-\varepsilon)}{\left[x^{2}+(y-\varepsilon)^{2}+z^{2}\right]^{3 / 2}}=\left.\frac{3 P}{2 \pi} \frac{1}{\left(x^{2}+z^{2}\right)} \frac{(y-\varepsilon)}{\left[x+(y-\varepsilon)^{2}+z^{2}\right]^{1 / 2}}\right|_{-b} ^{b} \\
\sigma=\frac{P}{2 \pi} \frac{z}{\left(x^{2}+y^{2}\right)}\left\{\frac{(y+b)}{\left[x^{2}+(y+b)^{2}\right]^{1 / 2}}-\frac{(y-b)}{\left[x^{2}+(y-b)^{2}+z^{2}\right]^{1 / 2}}\right\} \cdot(4.47)
\end{gathered}
$$

Компоненты напряжений определяются интегрированием соответствующих компонентов решения (2.40)-(2.45). 
Определение компонента $\sigma_{x}$ :

$$
\begin{aligned}
& \sigma_{x}=\frac{-3 P x^{2} z}{2 \pi} \int_{-b}^{b} \frac{d(y-\varepsilon)}{\left[x^{2}+(y-\varepsilon)^{2}+z^{2}\right]^{5 / 2}}= \\
& =-\left.\frac{3 P x^{2} z}{2 \pi\left(x^{2}+z^{2}\right)^{2}}\left\{\frac{(y-\varepsilon)}{\left[x^{2}+(y-\varepsilon)^{2}+z^{2}\right]^{1 / 2}}-\frac{1}{3} \frac{(y-\varepsilon)^{3}}{\left[x^{2}+(y-\varepsilon)^{2}+z^{2}\right]^{3 / 2}}\right\}\right|_{-b} ^{b} \\
& \sigma_{x}=\frac{3 P}{2 \pi} \frac{x^{2} z}{\left(x^{2}+z^{2}\right)^{2}}\left\{\frac{(y+b)}{\left[x^{2}+(y+b)^{2}+z^{2}\right]^{1 / 2}}-\frac{(y+b)^{3}}{3\left[x^{2}+(y+b)^{2}+z^{2}\right]^{3 / 2}}-\right. \\
& \left.-\frac{(y-b)}{\left[x^{2}+(y-b)^{2}+z^{2}\right]^{1 / 2}}+\frac{(y-b)^{3}}{3\left[x^{2}+(y-b)^{2}+z^{2}\right]^{3 / 2}}\right\} \\
& \underbrace{}_{i z}
\end{aligned}
$$

Рис. 4.5. Схема загружения полупространства равномерно распределенной вдоль линии нормальной к поверхности нагрузкой 
Определение компонента $\sigma_{y}$ :

$$
\begin{gathered}
\sigma_{y}=\frac{3 P}{2 \pi} z \int_{-b}^{b} \frac{(y-\varepsilon) d(y-\varepsilon)}{\left[x^{2}+(y-\varepsilon)^{2}+z^{2}\right]^{5 / 2}}= \\
=-\left.\frac{3 P}{2 \pi} \frac{z}{\left(x^{2}+z^{2}\right)^{2}}\left\{\frac{(y-\varepsilon)^{3}}{\left[x^{2}+(y-\varepsilon)^{2}+z^{2}\right]^{3 / 2}}\right\}\right|_{-b} ^{b} \\
\sigma_{y}=\frac{3 P}{2 \pi} \frac{z}{3\left(x^{2}+z^{2}\right)^{2}}\left\{\frac{(y+b)^{3}}{\left[x^{2}+(y+b)^{2}+z^{2}\right]^{\frac{3}{2}}}-\frac{(y-b)^{3}}{\left[x^{2}+(y-b)^{2}+z^{2}\right]^{\frac{3}{2}}}\right\} .(4.49)
\end{gathered}
$$

Определение компонента $\sigma_{z}$ :

$$
\begin{aligned}
& \sigma_{z}=-\frac{3 P z^{3}}{2 \pi} \int_{-b}^{b} \frac{d(y-\varepsilon)}{\left[x^{2}+(y-\varepsilon)^{2}+z^{2}\right]^{5 / 2}}=-\frac{3 P}{2 \pi} \frac{z^{3}}{\left(x^{2}+z^{2}\right)^{2}} \times \\
& \times\left.\left\{\frac{(y-\varepsilon)}{\left[x^{2}+(y-\varepsilon)^{2}+z^{2}\right]}-\frac{1}{3} \frac{(y-\varepsilon)^{3}}{\left[x^{2}+(y-\varepsilon)^{2}+z^{2}\right]^{3 / 2}}\right\}\right|_{-b} ^{b} \\
& \sigma_{z}=\frac{3 P}{2 \pi} \frac{z^{3}}{\left(x^{2}+z^{2}\right)^{2}}\left\{\frac{(y+b)}{\left[x^{2}+(y+b)^{2}+z^{2}\right]^{\frac{1}{2}}}-\frac{1}{3} \frac{(y+b)^{3}}{\left[x^{2}+(y+b)^{2}+z^{2}\right]^{\frac{3}{2}}}-\right. \\
& \left.-\frac{(y-b)}{\left[x^{2}+(y-b)^{2}+z^{2}\right]^{\frac{1}{2}}}+\frac{1}{3} \frac{(y-b)^{3}}{\left[x^{2}+(y-b)^{2}+z^{2}\right]^{\frac{3}{2}}}\right\}
\end{aligned}
$$


Определение компонента $\tau_{x y}$ :

$$
\begin{gathered}
\tau_{x y}=-\frac{3 P}{2 \pi} x z \int_{-b}^{b} \frac{(y-\varepsilon) d(y-\varepsilon)}{\left[x^{2}+(y+\varepsilon)^{2}+z^{2}\right]^{5 / 2}}=-\left.\frac{3 P}{2 \pi} x z \frac{1}{3} \frac{1}{\left[x^{2}+(y-\varepsilon)^{2}+z^{2}\right]^{3 / 2}}\right|_{-b} ^{b} \\
\tau_{x y}=\frac{P}{2 \pi} x z\left\{\frac{1}{\left[x^{2}+(y+b)^{2}+z^{2}\right]^{3 / 2}}-\frac{1}{\left[x^{2}+(y-b)^{2}+z^{2}\right]^{3 / 2}}\right\} \cdot(4.51)
\end{gathered}
$$

Определение компонента $\tau_{y z}$ :

$$
\begin{gathered}
\tau_{y z}=-\frac{3 P}{2 \pi} z^{2} \int_{-b}^{b} \frac{(y-\varepsilon) d(y-\varepsilon)}{\left[x^{2}+(y+\varepsilon)^{2}+z^{2}\right]^{5 / 2}}=-\left.\frac{3 P}{2 \pi} \frac{z^{2}}{3} \frac{1}{\left[x^{2}+(y-\varepsilon)^{2}+z^{2}\right]^{3 / 2}}\right|_{-b} ^{b} \\
\tau_{y z}=\frac{P}{2 \pi} z^{2}\left\{\frac{1}{\left[x^{2}+(y+b)^{2}+z^{2}\right]^{3 / 2}}-\frac{1}{\left[x^{2}+(y-b)^{2}+z^{2}\right]^{3 / 2}}\right\} .
\end{gathered}
$$

Определение компонента $\tau_{z x}$ :

$$
\begin{aligned}
\tau_{z x}=-\frac{3 P}{2 \pi} z^{2} x \int_{-b}^{b} \frac{d(y-\varepsilon)}{\left[x^{2}+(y-\varepsilon)^{2}+z^{2}\right]^{5 / 2}}=-\frac{3 P}{2 \pi} \frac{z^{2} x}{\left(x^{2}+z^{2}\right)^{2}} \times \\
\times\left\{\frac{(y-\varepsilon)}{\left[x^{2}+(y-\varepsilon)^{2}+z^{2}\right]^{1 / 2}}-\frac{1}{3} \frac{(y+b)^{3}}{\left[x^{2}+(y+b)^{2}+z^{2}\right]^{3 / 2}}\right\}_{-b}^{b} ;
\end{aligned}
$$




$$
\begin{array}{r}
\tau_{z x}=\frac{3 P}{2 \pi} \frac{z^{2} x}{\left(x^{2}+z^{2}\right)^{2}}\left\{\frac{(y+b)}{\left[x^{2}+(y+b)^{2}+z^{2}\right]^{\frac{1}{2}}}-\frac{1}{3} \frac{(y+b)^{3}}{\left[x^{2}+(y+b)^{2}+z^{2}\right]^{\frac{3}{2}}}-\right. \\
\left.-\frac{(y-b)}{\left[x^{2}+(y-b)^{2}+z^{2}\right]^{\frac{1}{2}}}+\frac{(y-b)^{3}}{\left[x^{2}+(y-b)^{2}+z^{2}\right]^{3}}\right\} .
\end{array}
$$

Для определения перемещений, вызванных сдвиговыми деформациями, сначала находим частные производные функции давления, а сами перемещения записываем с использованием формул (3.28)(3.30). В результате будем иметь:

$$
\begin{aligned}
& U^{c}=K^{c} \frac{P}{2 \pi} \frac{x z}{\left(x^{2}+z^{2}\right)}\left\{\frac{2}{\left(x^{2}+z^{2}\right)} \times\right. \\
& \times\left[\frac{(y+b)}{\left[x^{2}+(y+b)^{2}+z^{2}\right]^{1 / 2}}-\frac{(y-b)}{\left[x^{2}+(y-b)^{2}+z^{2}\right]^{1 / 2}}\right]+ \\
&\left.+\left[\frac{(y+b)}{\left[x^{2}+(y+b)^{2}+z^{2}\right]^{3 / 2}}-\frac{(y-b)}{\left[x^{2}+(y-b)^{2}+z^{2}\right]^{3 / 2}}\right]\right\} \\
& V^{\mathrm{c}}=-K^{\mathrm{c}} \frac{P}{2 \pi} z\left\{\frac{1}{\left[x^{2}+(y+b)^{2}+z^{2}\right]^{3 / 2}}-\frac{1}{\left[x^{2}+(y-b)^{2}+z^{2}\right]^{3 / 2}}\right\} \\
& W^{\mathrm{c}}=-K^{\mathrm{c}} \frac{P}{2 \pi} \frac{1}{\left(x^{2}+z^{2}\right)}\left\{\frac{\left(x^{2}-z^{2}\right)}{\left(x^{2}+z^{2}\right)} \times\right.
\end{aligned}
$$




$$
\begin{aligned}
& \times\left[\frac{(y+b)}{\left[x^{2}+(y+b)^{2}+z^{2}\right]^{1 / 2}}-\frac{(y-b)}{\left[x^{2}+(y-b)^{2}+z^{2}\right]^{1 / 2}}\right]- \\
& \left.-z^{2}\left[\frac{(y+b)}{\left[x^{2}+(y+b)^{2}+z^{2}\right]^{3 / 2}}-\frac{(y-b)}{\left[x^{2}+(y-b)^{2}+z^{2}\right]^{3 / 2}}\right]\right\} .
\end{aligned}
$$

Определение вертикальных перемещений от изменения плотности среды выполняется интегрированием (4.47) с учетом (3.19):

$$
\begin{gathered}
W^{0}=K^{0} \frac{P}{2 \pi} \int_{z}^{\infty}\left\{\frac{z}{\left(x^{2}+z^{2}\right)} \frac{(y+b)}{\left[x^{2}+(y+b)^{2}+z^{2}\right]^{1 / 2}}-\right. \\
\left.-\frac{z}{\left(x^{2}+z^{2}\right)} \frac{(y-b)}{\left[x^{2}+(y-b)^{2}+z^{2}\right]^{1 / 2}}\right\} d z .
\end{gathered}
$$

В целях сокращения записей интегрируем выражение:

$$
\int \frac{z d z}{\left(x^{2}+z^{2}\right)\left(x^{2}+y^{2}+z^{2}\right)^{1 / 2}}
$$

в котором выполняем подстановку:

$$
t^{2}=x^{2}+y^{2}+z^{2},
$$

откуда

$$
\begin{gathered}
z^{2}=t^{2}-x^{2}-y^{2} \\
t d t=z d z
\end{gathered}
$$


Интегрирование выполняется по новой переменной $t$ :

$$
\int \frac{t d t}{\left(x^{2}+t^{2}-x^{2}-y^{2}\right) t}=-\int \frac{t d t}{\left(y^{2}-t^{2}\right) t}=-\frac{1}{2 y} \ln \left(\frac{y+t}{y-t}\right) .
$$

Выполнив обратную замену, имеем:

$$
\begin{aligned}
\int_{z}^{\infty} \frac{z d z}{\left(x^{2}+y^{2}\right)\left(x^{2}+y^{2}+z^{2}\right)^{1 / 2}}=-\left.\frac{1}{2 y} \ln \left[\frac{y+\left(x^{2}+y^{2}+z^{2}\right)^{1 / 2}}{y-\left(x^{2}+y^{2}+z^{2}\right)^{1 / 2}}\right]\right|_{z} ^{\infty}= \\
=\frac{1}{2 y} \ln \left[\frac{y+\left(x^{2}+y^{2}+z^{2}\right)^{1 / 2}}{y-\left(x^{2}+y^{2}+z^{2}\right)^{1 / 2}}\right] .
\end{aligned}
$$

Окончательно после соответствующей подстановки в (4.57) имеем:

$$
\begin{aligned}
W^{0}=K^{0} \frac{P}{4 \pi}\left\{\frac{1}{(y+b)} \ln \right. & {\left[\frac{(y+b)+\left[x^{2}+(y+b)^{2}+z^{2}\right]^{1 / 2}}{(y+b)-\left[x^{2}+(y+b)^{2}+z^{2}\right]^{1 / 2}}\right]-} \\
& \left.-\frac{1}{y-b} \ln \left[\frac{(y-b)+\left[x^{2}+(y-b)^{2}+z^{2}\right]^{1 / 2}}{(y-b)-\left[x^{2}+(y-b)^{2}+z^{2}\right]^{1 / 2}}\right]\right\} .
\end{aligned}
$$

Следует отметить, что предельный переход в формулах (4.47)(4.53) путем $-\infty \leq b \leq \infty$ дает напряженное состояние полупространства при его плоском деформировании, когда нормальная нагрузка равномерно распределена на поверхности вдоль бесконечной линии:

$$
\sigma=\frac{P}{\pi} \frac{z}{\left(x^{2}+z^{2}\right)}
$$




$$
\begin{gathered}
\sigma_{x}=\frac{2 P}{\pi} \frac{x^{2} z}{\left(x^{2}+z^{2}\right)^{2}} ; \\
\sigma_{y}=\sigma=\frac{P}{\pi} \frac{z}{\left(x^{2}+z^{2}\right)} ; \\
\sigma_{z}=\frac{2 P}{\pi} \frac{z^{3}}{\left(x^{2}+z^{2}\right)^{2}} ; \\
\tau_{x y}=\tau_{y z}=0 \\
\tau_{z t}=\frac{2 P}{\pi} \frac{z^{2} x}{\left(x^{2}+z^{2}\right)^{2}} .
\end{gathered}
$$

Перемещения, вызванные сдвиговыми деформациями, в соответствии с (3.28)-(3.30) будут:

$$
\begin{gathered}
U^{\mathrm{c}}=-K^{\mathrm{c}} \frac{\partial \sigma}{\partial x}=\frac{P}{\pi} \frac{2 x z}{\left(x^{2}+z^{2}\right)^{2}} ; \\
W^{\mathrm{c}}=-K^{\mathrm{c}} \frac{\partial \sigma}{\partial z}=-\frac{P}{\pi} \frac{\left(x^{2}-z^{2}\right)}{\left(x^{2}+z^{2}\right)^{2}} .
\end{gathered}
$$

Перемещения, вызванные изменением плотности среды, направлены параллельно линии действия внешней нагрузки и определяются интегрированием:

$$
\begin{gathered}
W^{0}=\int_{z_{1}}^{z_{2}} \varepsilon_{z} d z=K^{0} \frac{P}{\pi} \int_{z_{1}}^{z_{2}} \sigma d z=K^{0} \frac{P}{\pi} \int_{z_{1}}^{z_{2}} \frac{z d z}{\left(x^{2}+z^{2}\right)} \\
W^{0}=\left.K^{0} \frac{P}{2 \pi} \ln \left(x^{2}+z^{2}\right)\right|_{z_{1}} ^{z_{2}}
\end{gathered}
$$


или

$$
W^{0}=K^{0} \frac{P}{2 \pi} \ln \left|\frac{x^{2}+z_{2}^{2}}{x^{2}+z_{1}^{2}}\right| \text {; }
$$

Поскольку в (4.66) при неограниченном возрастании координаты $z$ вертикальное перемещение $W^{0}$ также растет неограниченно, то с ее помощью можно определять только величину сжатия конкретного слоя упругой среды конечной толщины.

Таким образом, представлено полное решение задачи о напряженно-деформированном состоянии упругого полупространства при его нагружении равномерно распределенной нагрузкой по линии конечной и бесконечной длинны.

\subsection{5. Нормальная нагрузка, равномерно распределенная на полосе постоянной ширины конечной и бесконечной длины}

Рассмотрим сначала задачу, когда нагрузка равномерной интенсивности $p\left(H / \mathrm{M}^{2}\right)$ распределена по площади прямоугольника (рис. 4.6). Граничными условиями для гармонической функции давления будут:

в пределах загруженной площадки $z=0, \sigma=p$,

за пределами загруженной площадки $z=0, \sigma=0$;

$$
z \neq 0, R \rightarrow \infty, \sigma \rightarrow 0
$$

Функция давления, удовлетворяющая этим условиям, получена в [25] при исследовании мгновенных напоров в водонасыщенном грунте, возникающих в результате его быстрого загружения аналогичной нагрузкой:

$\sigma=\frac{p}{2 \pi}\left\{-\operatorname{arctg} \frac{z\left[(x+a)^{2}+(y+b)^{2}+z^{2}\right]^{\frac{1}{2}}}{(x+a)(y+b)}+\right.$ 


$$
+\operatorname{arctg} \frac{z\left[(x+a)^{2}+(y-b)^{2}+z^{2}\right]^{\frac{1}{2}}}{(x+a)(y-b)}+\operatorname{arctg} \frac{z\left[(x-a)^{2}+(y+b)^{2}+z^{2}\right]^{\frac{1}{2}}}{(x-a)(y+b)}-
$$

$$
\left.-\operatorname{arctg} \frac{z\left[(x-a)^{2}+(y-b)^{2}+z^{2}\right]^{\frac{1}{2}}}{(x-a)(y-b)}\right\} .
$$

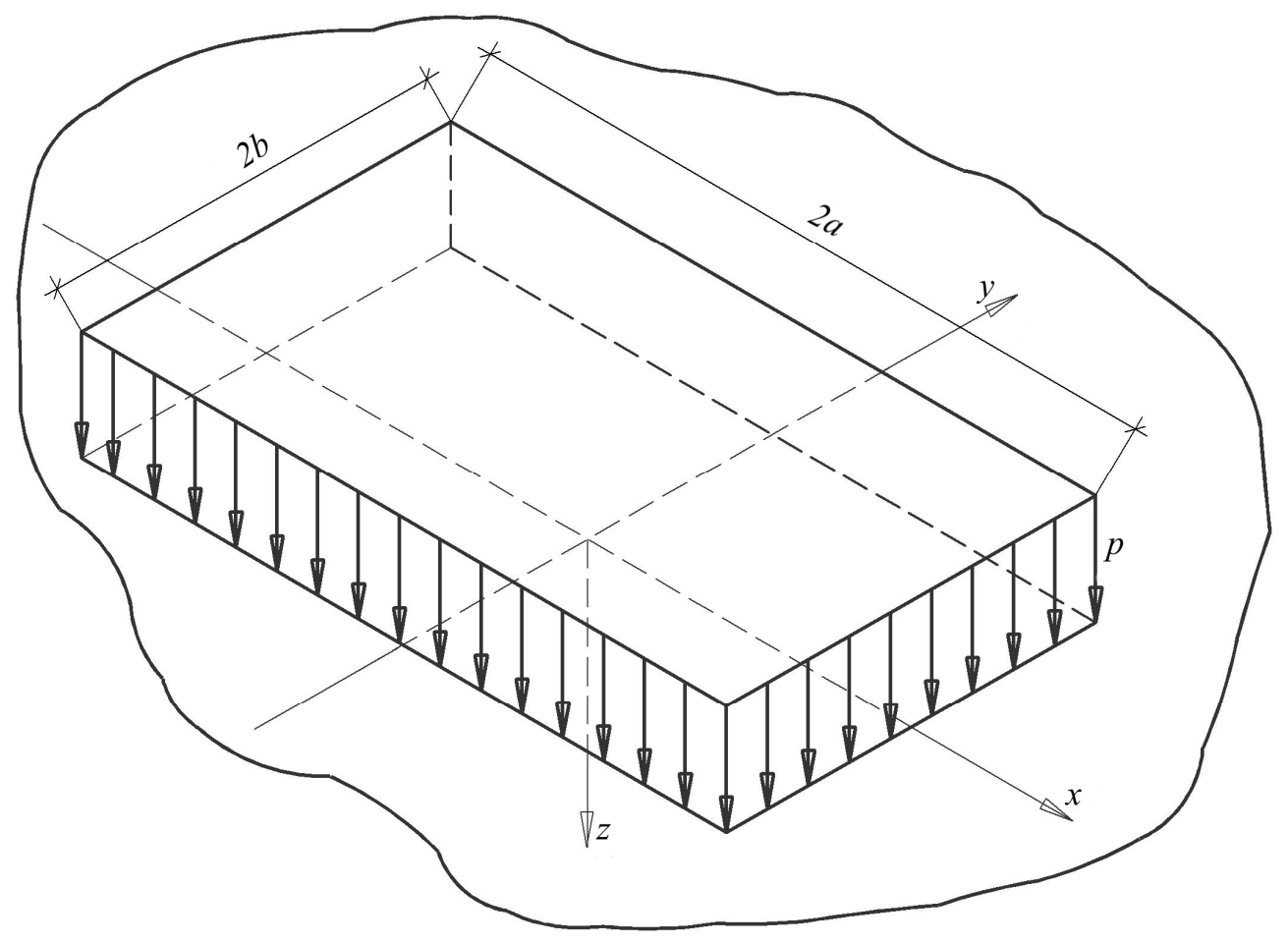

Рис. 4.6. Схема загружения полупространства равномерной нагрузкой по площади прямоугольника

Эту же функцию можно получить путем интегрирования функции давления для сосредоточенной силы:

$$
\sigma=\frac{P}{2 \pi} \int_{-a-b}^{a} \int_{[}^{b} \frac{z d(x-\varepsilon) d(y-\xi)}{\left[(x-\varepsilon)^{2}+(y-\xi)^{2}+z^{2}\right]^{3 / 2}}=
$$




$$
\begin{gathered}
=\left.\frac{p z}{2 \pi} \int_{-b}^{b} \frac{(x-\varepsilon) z d(y-\xi)}{\left[(y-\xi)^{2}+z^{2}\right]\left[(x-\varepsilon)^{2}+(y-\xi)^{2}+z^{2}\right]^{1 / 2}}\right|_{-a} ^{a}= \\
=\left.\frac{P}{2 \pi} \operatorname{arctg} \frac{(x-\varepsilon)(y-\xi)}{z\left[(x-\varepsilon)^{2}+(y-\xi)^{2}+z^{2}\right]}\right|_{-a-b} ^{a},
\end{gathered}
$$

а после подстановки пределов интегрирования получаем:

$$
\begin{gathered}
\sigma=\frac{P}{2 \pi}\left\{\operatorname{arctg} \frac{(x-a)(y-b)}{z\left[(x-a)^{2}+(y-b)^{2}+z^{2}\right]^{1 / 2}}+\right. \\
+\operatorname{arctg} \frac{(x+a)(y-b)}{z\left[(x+a)^{2}+(y-b)^{2}+z^{2}\right]^{1 / 2}}+\operatorname{arctg} \frac{(x-a)(y+b)}{z\left[(x-a)^{2}+(y+b)^{2}+z^{2}\right]^{1 / 2}}+ \\
\left.+\operatorname{arctg} \frac{(x+a)(y+b)}{z\left[(x+a)^{2}+(y+b)^{2}+z^{2}\right]^{1 / 2}}\right\} .(4.68)
\end{gathered}
$$

Функции (4.67) и (4.68) дают одинаковый результат, если в (4.67) арктангенс брать в пределах от 0 до $\pi$, в функции же (4.68) он берется как обычно: при изменении ее в промежутке от $-\infty$ до $+\infty$ арктангенс изменяется от $-\frac{\pi}{2}$ до $+\frac{\pi}{2}$.

Для определения компонентов напряжений в этой задаче есть два пути.

1. Можно воспользоваться соотношениями В. А. Флорина (3.37) для пространственных задач с плоской поверхностью при действии на них нормальной нагрузки.

2. Можно интегрировать компоненты напряжений, полученные для полупространства при действии сосредоточенной силы, направленной по нормали к его поверхности (2.40)-(2.45). 
Однако, поскольку в традиционной постановке эта задача была решена [27], то воспользуемся этим решением для определения компонентов напряжений среды в рассматриваемой задаче, приняв в нем коэффициент бокового расширения $v=0,5$. В результате получим:

$$
\begin{aligned}
& \sigma_{x}=\frac{p}{2 \pi}\left\{\frac{(x+a)(y+b) z}{\left[(x+a)^{2}+z^{2}\right]\left[(x+a)^{2}+(y+b)^{2}+z^{2}\right]^{1 / 2}}-\right. \\
& -\frac{(x-a)(y-b) z}{\left[(x-a)^{2}+z^{2}\right]\left[(x-a)^{2}+(y-b)^{2}+z^{2}\right]^{1 / 2}}- \\
& +\frac{-\frac{(x+a)(y-b) z}{\left[(y-b)^{2}+z^{2}\right]\left[(x+a)^{2}+(y-b)^{2}+z^{2}\right]^{1 / 2}}}{\left[(y+b)^{2}+z^{2}\right]\left[(x-a)^{2}+(y+b)^{2}+z^{2}\right]^{1 / 2}-} \\
& \left.\begin{array}{c}
-\operatorname{arctg} \frac{z}{z}=\frac{(x-a)(y+b) z}{2 \pi}\left\{\frac{\left.(x-a)^{2}+(y-b)^{2}+z^{2}\right]^{\frac{1}{2}}}{(x-a)(y-b)}+\operatorname{arctg} \frac{z\left[(x-a)^{2}+(y+b)^{2}+z^{2}\right]^{\frac{1}{2}}}{(x-a)(y+b)}-\right. \\
-\operatorname{arctg} \frac{z\left[(x+a)^{2}+(y+b)^{2}+z^{2}\right]^{\frac{1}{2}}}{(x+a)(y+b)}+\operatorname{arctg} \frac{z\left[(x+a)^{2}+(y-b)^{2}+z^{2}\right]^{\frac{1}{2}}}{(x+a)(y-b)}
\end{array}\right\}
\end{aligned}
$$




$$
\begin{aligned}
& -\frac{(x+a)(y+b) z}{\left[(y+b)^{2}+z^{2}\right]\left[(x+a)^{2}+(y+b)^{2}+z^{2}\right]^{1 / 2}}- \\
& -\frac{(x-a)(y-b) z}{\left[(y-b)^{2}+z^{2}\right]\left[(x-a)^{2}+(y-b)^{2}+z^{2}\right]^{1 / 2}}+ \\
& +\frac{(x-a)(y+b) z}{\left[(y+b)^{2}+z^{2}\right]\left[(x-a)^{2}+(y+b)^{2}+z^{2}\right]^{1 / 2}}- \\
& -\operatorname{arctg} \frac{z\left[(x-a)^{2}+(y-b)^{2}+z^{2}\right]^{\frac{1}{2}}}{(x-a)(y-b)}+\operatorname{arctg} \frac{z\left[(x-a)^{2}+(y+b)^{2}+z^{2}\right]^{\frac{1}{2}}}{(x-a)(y+b)}- \\
& \left.-\operatorname{arctg} \frac{z\left[(x+a)^{2}+(y+b)^{2}+z^{2}\right]^{\frac{1}{2}}}{(x+a)(y+b)}+\operatorname{arctg} \frac{z\left[(x+a)^{2}+(y-b)^{2}+z^{2}\right]^{\frac{1}{2}}}{(x+a)(y-b)}\right\} \\
& \sigma_{z}=\frac{p}{2 \pi}\left\{\frac{z(x-a)(y-b)}{\left[(x-a)^{2}+z^{2}\right]\left[(y-b)^{2}+z^{2}\right]} \frac{\left[(x-a)^{2}+(y-b)^{2}+2 z^{2}\right]}{\left[(x-a)^{2}+(y-b)^{2}+z^{2}\right]^{\frac{1}{2}}}-\right. \\
& -\frac{z(x-a)(y+b)}{\left[(x-a)^{2}+z^{2}\right]\left[(y+b)^{2}+z^{2}\right]} \frac{\left[(x-a)^{2}+(y+b)^{2}+2 z^{2}\right]}{\left[(x-a)^{2}+(y+b)^{2}+z^{2}\right]^{1 / 2}}- \\
& -\frac{z(x+a)(y-b)}{\left[(x+a)^{2}+z^{2}\right]\left[(y-b)^{2}+z^{2}\right]} \frac{\left[(x+a)^{2}+(y-b)^{2}+2 z^{2}\right]}{\left[(x+a)^{2}+(y-b)^{2}+z^{2}\right]^{1 / 2}}+
\end{aligned}
$$




$$
\begin{aligned}
& +\frac{z(x+a)(y+b)}{\left[(x+a)^{2}+z^{2}\right]\left[(y+b)^{2}+z^{2}\right]} \frac{\left[(x+a)^{2}+(y+b)^{2}+2 z^{2}\right]}{\left[(x+a)^{2}+(y+b)^{2}+z^{2}\right]^{1 / 2}}- \\
& -\operatorname{arctg} \frac{z\left[(x-a)^{2}+(y-b)^{2}+z^{2}\right]^{1 / 2}}{(x-a)(y-b)}+\operatorname{arctg} \frac{z\left[(x-a)^{2}+(y+b)^{2}+z^{2}\right]^{1 / 2}}{(x-a)(y+b)}+ \\
& \left.+\operatorname{arctg} \frac{z\left[(x+a)^{2}+(y-b)^{2}+z^{2}\right]}{(x+a)(y-b)}-\operatorname{arctg} \frac{z\left[(x+a)^{2}+(y+b)^{2}+z^{2}\right]^{1 / 2}}{(x+a)(y+b)}\right\} \\
& \tau_{x y}=\frac{p}{2 \pi}\left\{\frac{z}{\left[(x+a)^{2}+(y-b)^{2}+z^{2}\right]^{1 / 2}}-\frac{z}{\left[(x-a)^{2}+(y+b)^{2}+z^{2}\right]^{1 / 2}}-\right. \\
& \left.-\frac{z}{\left[(x+a)^{2}+(y-b)^{2}+z^{2}\right]^{1 / 2}}+\frac{z}{\left[(x+a)^{2}+(y+b)^{2}+z^{2}\right]^{1 / 2}}\right\} \\
& \tau_{y z}=\frac{p z^{2}}{2 \pi}\left\{\frac{(x+a)}{\left[(y-b)^{2}+z^{2}\right]\left[(x+a)^{2}+(y-b)^{2}+z\right]^{1 / 2}}-\right. \\
& -\frac{(x-a)}{\left[(y-b)^{2}+z^{2}\right]\left[(x-a)^{2}+(y-b)^{2}+z\right]^{1 / 2}}- \\
& -\frac{(x+a)}{\left[(y+b)^{2}+z^{2}\right]\left[(x+a)^{2}+(y+b)^{2}+z\right]^{1 / 2}}+
\end{aligned}
$$




$$
\begin{gathered}
\tau_{z x}=\frac{p z^{2}}{2 \pi}\left\{\frac{(x-a)}{\left[(y+b)^{2}+z\right]\left[(x-a)^{2}+(y+b)^{2}+z\right]^{1 / 2}}\right\} \\
-\frac{(y+b)}{\left[(x+a)^{2}+z^{2}\right]\left[(x-a)^{2}+(y+b)^{2}+z\right]^{1 / 2}}- \\
(y+b)+\frac{(y-b)}{\left[(x+a)^{2}+z^{2}\right]\left[(x+a)^{2}+(y-b)^{2}+z\right]^{1 / 2}}- \\
\left.+\frac{\left[(x+a)^{2}+(y+b)^{2}+z\right]^{1 / 2}+}{\left[(x+a)^{2}+z^{2}\right]\left[(x+a)^{2}+(y-b)^{2}+z\right]^{1 / 2}}\right\}
\end{gathered}
$$

Для определения компонентов перемещений, вызванных чистым сдвигом в упругой среде, находятся частные производные функции давления (4.67). В результате имеем:

$$
\begin{aligned}
& \frac{\partial \sigma}{\partial x}=\frac{p}{2 \pi}\left\{\frac{(y+b)^{2} z\left[(y+b)^{2}+z\right]}{\left[(x+a)^{2}+(y+b)^{2}+z\right]^{1 / 2}} \times\right. \\
& \times \frac{1}{(x+a)^{2}(y+b)^{2}+z^{2}\left[(x+a)^{2}+(y+b)^{2}+z\right]}+\frac{(y-b)^{2} z\left[(y-b)^{2}+z\right]}{\left[(x+a)^{2}+(y-b)^{2}+z\right]^{\frac{1}{2}}} \times
\end{aligned}
$$




$$
\begin{aligned}
& \times \frac{1}{(x+a)^{2}(y-b)^{2}+z^{2}\left[(x+a)^{2}+(y-b)^{2}+z\right]}+\frac{(y+b) z\left[(y+b)^{2}+z^{2}\right]}{\left[(x-a)^{2}+(y+b)^{2}+z\right]^{\frac{1}{2}}} \times \\
& \times \frac{1}{(x-a)^{2}(y+b)^{2}+z^{2}\left[(x-a)^{2}+(y+b)^{2}+z^{2}\right]}+\frac{(y-b) z\left[(y-b)^{2}+z^{2}\right]}{\left[(x-a)^{2}+(y-b)^{2}+z\right]^{\frac{1}{2}}} \times \\
& \left.\times \frac{1}{(x-a)^{2}(y-b)^{2}+z^{2}\left[(x-a)^{2}+(y-b)^{2}+z^{2}\right]}\right\} \\
& \frac{\partial \sigma}{\partial y}=\frac{p}{2 \pi}\left\{\frac{(x+a) z\left[(x+a)^{2}+z\right]}{\left[(x+a)^{2}+(y+b)^{2}+z\right]^{1 / 2}} \times\right. \\
& \times \frac{1}{(x+a)^{2}(y+b)^{2}+z^{2}\left[(x+a)^{2}+(y+b)^{2}+z\right]}+\frac{(x+a) z\left[(x+a)^{2}+z^{2}\right]}{\left[(x+a)^{2}+(y-b)^{2}+z^{2}\right]^{\frac{1}{2}}} \times \\
& \times \frac{1}{(x+a)^{2}(y-b)^{2}+z^{2}\left[(x+a)^{2}+(y-b)^{2}+z^{2}\right]}+\frac{(x-a) z\left[(x-a)^{2}+z^{2}\right]}{\left[(x-a)^{2}+(y+b)^{2}+z^{2}\right]^{\frac{1}{2}}} \times \\
& \times \frac{1}{(x-a)^{2}(y+b)^{2}+z^{2}\left[(x-a)^{2}+(y+b)^{2}+z^{2}\right]}+ \\
& +\frac{1}{(x-a)^{2}(y-b)^{2}+z^{2}\left[(x-a)^{2}+(y-b)^{2}+z^{2}\right]} \times
\end{aligned}
$$




$$
\left.\times \frac{(x-a) z\left[(x-a)^{2}+z^{2}\right]}{\left[(x-a)^{2}+(y-b)^{2}+z\right]^{1 / 2}}\right\}
$$

$$
\begin{aligned}
\frac{\partial \sigma}{\partial z}=-\frac{p}{2 \pi}\{ & \frac{(x+a)(y+b)\left[(x+a)^{2}+(y+b)^{2}+2 z^{2}\right]}{\left[(x+a)^{2}+(y+b)^{2}+z^{2}\right]^{1 / 2}} \times \\
& \times \frac{1}{(x+a)^{2}(y+b)^{2}+z^{2}\left[(x+a)^{2}+(y+b)^{2}+z^{2}\right]}+ \\
& +\frac{(x+a)(y-b)\left[(x+a)^{2}+(y-b)^{2}+2 z^{2}\right]}{\left[(x+a)^{2}+(y-b)^{2}+z^{2}\right]^{1 / 2}} \times
\end{aligned}
$$

$$
\begin{gathered}
\times \frac{1}{(x+a)^{2}(y-b)^{2}+z^{2}\left[(x+a)^{2}+(y-b)^{2}+z^{2}\right]}+ \\
+\frac{(x-a)(y+b)\left[(x-a)^{2}+(y+b)+2 z^{2}\right]}{\left[(x-a)^{2}+(y+b)^{2}+z^{2}\right]^{1 / 2}} \times \\
\times \frac{1}{(x-a)^{2}(y+b)^{2}+z^{2}\left[(x-a)^{2}+(y+b)^{2}+z^{2}\right]}
\end{gathered}
$$$$
+\frac{(x-a)(y-b)\left[(x-a)^{2}+(y-b)+2 z^{2}\right]}{\left[(x-a)^{2}+(y-b)^{2}+z^{2}\right]^{1 / 2}} \times
$$

$$
\left.\times \frac{1}{(x-a)^{2}(y-b)^{2}+z^{2}\left[(x-a)^{2}+(y-b)^{2}+z^{2}\right]}\right\} .
$$


Теперь перемещения точек среды, вызванные чистым сдвигом, в сокращенной форме записи будут:

$$
\begin{aligned}
& U^{\mathrm{c}}=-K^{\mathrm{c}} \frac{\partial \sigma}{\partial x} \\
& V^{\mathrm{c}}=-K^{\mathrm{c}} \frac{\partial \sigma}{\partial y} ; \\
& W^{\mathrm{c}}=-K^{\mathrm{c}} \frac{\partial \sigma}{\partial z} .
\end{aligned}
$$

Перемещения точек, вызванные изменением плотности среды, согласно (3.19) будут протекать только по вертикали, то есть

$$
U^{0}=V^{0}=0,
$$

а вертикальные перемещения будут

$$
W^{0}=\int_{\infty}^{z} K^{0} \varepsilon_{z}^{0} d z=K^{0} \int_{\infty}^{z} \sigma d z .
$$

Интегрированные функции (4.67) соответственно (4.69) в полных записях довольно трудоемкие, поэтому мы будем интегрировать один член этой формулы, записав его сокращенно:

$$
F=\int \operatorname{arctg} \frac{z\left(x^{2}+y^{2}+z^{2}\right) d z}{x y},
$$

а затем приведем результат в развернутом виде.

Интегрирование (4.70) выполняется по частям:

$$
u=\operatorname{arctg} \frac{1}{x y}\left[z^{2}\left(x^{2}+y^{2}+z^{2}\right)\right] ;
$$




$$
\begin{gathered}
d u=\frac{2 z\left(x^{2}+y^{2}\right)+4 z^{3}}{1+\frac{z^{2}\left(x^{2}+y^{2}+z^{2}\right)}{x^{2}+y^{2}} x y 2\left[z^{2}\left(x^{2}+y^{2}+z^{2}\right)\right]^{1 / 2}} \\
d u=\frac{x y\left(x^{2}+y^{2}+2 z^{2}\right)}{\left[x^{2} y^{2}+z^{2}\left(x^{2}+y^{2}+z^{2}\right)\right]\left(x^{2}+y^{2}+z^{2}\right)^{1 / 2}} d z ; \\
d v=d z ; v=z ; \\
-F=u v-\int v d u=z \operatorname{arctg} \frac{z\left(x^{2}+y^{2}+z^{2}\right)^{1 / 2}}{x y} \\
-\int z \frac{x y\left(x^{2}+y^{2}+2 z^{2}\right)}{\left[x^{2} y^{2}+z^{2}\left(x^{2}+y^{2}+z^{2}\right)\right]\left(x^{2}+y^{2}+z^{2}\right)^{1 / 2}} d z .
\end{gathered}
$$

Вводится новый аргумент:

$$
t^{2}=\left(x^{2}+y^{2}+z^{2}\right)
$$

при этом

$$
2 t d t=2 z d z ; t d t=z d z,
$$

тогда:

$$
\begin{aligned}
& \int z \frac{x y\left(x^{2}+y^{2}+2 z^{2}\right)}{\left[x^{2} y^{2}+z^{2}\left(x^{2}+y^{2}+z^{2}\right)\right]\left(x^{2}+y^{2}+z^{2}\right)} d z= \\
& \quad=\int \frac{x y\left(2 t^{2}-x^{2}-y^{2}\right) t d t}{\left[x^{2} y^{2}+t^{2}\left(t^{2}-x^{2}-y^{2}\right) t^{2}\right] t}=\int \frac{x y\left(2 t^{2}-x^{2}-y^{2}\right)}{t^{4}-t^{2}\left(x^{2}+y^{2}\right)+x^{2} y^{2}} d t=
\end{aligned}
$$




$$
\begin{gathered}
=x y \int \frac{\left(t^{2}-x^{2}\right)+\left(t^{2}-y^{2}\right)}{\left(t^{2}-x^{2}\right)\left(t^{2}-y^{2}\right)} d t=x y\left[\frac{\left(t^{2}-x^{2}\right)}{\left(t^{2}-x^{2}\right)\left(t^{2}-y^{2}\right)} d t+\right. \\
\left.+\int \frac{\left(t^{2}-y^{2}\right) d t}{\left(t^{2}-x^{2}\right)\left(t^{2}-y^{2}\right)}\right]=x y \int \frac{d t}{\left(t^{2}-y^{2}\right)}+x y \int \frac{d t}{\left(t^{2}-x^{2}\right)}= \\
=\frac{x y}{y^{2}} \int \frac{d t}{\left(1+\frac{t^{2}}{y^{2}}\right)}-\frac{x y}{x^{2}} \int \frac{d t}{\left(1-\frac{t^{2}}{x^{2}}\right)}=x \frac{1}{2} \ln \left|\frac{(1+t / y) \mid}{(1+t / y)}\right|-y \frac{1}{2} \ln \left|\frac{(1+t / x)}{(1+t / y) \mid}\right|= \\
=-\frac{x}{2} \ln \left|\frac{(y+t)}{(y-t)}\right|-\frac{y}{2} \ln \left|\frac{(x+t)}{(x-t)}\right| .
\end{gathered}
$$

Возвращаясь к аргументу $z$, окончательно имеем:

$$
\begin{aligned}
F=z \operatorname{arctg} \frac{z\left(x^{2}+y^{2}+z^{2}\right)^{1 / 2}}{x y}+ \\
+\frac{x}{2} \ln \left|\frac{y+\left(x^{2}+y^{2}+z^{2}\right)^{1 / 2}}{y-\left(x^{2}+y^{2}+z^{2}\right)^{1 / 2}}\right|+\frac{y}{2} \ln \left|\frac{x+\left(x^{2}+y^{2}+z^{2}\right)^{1 / 2}}{x-\left(x^{2}+y^{2}+z^{2}\right)^{1 / 2}}\right| .
\end{aligned}
$$

Теперь запишем результат интегрирования (4.69) в развернутом виде:

$$
\begin{aligned}
W^{0}=\frac{p}{2 \pi}\{ & -z \operatorname{arctg} \frac{z\left[(x+a)^{2}+(y+b)^{2}+z^{2}\right]^{1 / 2}}{(x+a)(y+b)}- \\
& -\frac{(x+a)}{2} \ln \left|\frac{(y+b)+\left[(x+a)^{2}+(y+b)^{2}+z^{2}\right]^{1 / 2}}{(y+b)-\left[(x+a)+(y+b)^{2}+z^{2}\right]^{1 / 2}}\right|-
\end{aligned}
$$




$$
\begin{aligned}
& -\frac{(y+b)}{2} \ln \left|\frac{(x+a)+\left[(x+a)^{2}+(y+b)^{2}+z^{2}\right]^{1 / 2}}{(x+a)-\left[(x+a)+(y+b)^{2}+z^{2}\right]^{1 / 2}}\right|+ \\
& +z \operatorname{arctg} \frac{z\left[(x+a)^{2}+(y-b)^{2}+z^{2}\right]^{1 / 2}}{(x+a)(y-b)}+ \\
& +\frac{(x+a)}{2} \ln \left|\frac{(y-b)+\left[(x+a)^{2}+(y-b)^{2}+z^{2}\right]^{1 / 2}}{(y-b)-\left[(x+a)+(y-b)^{2}+z^{2}\right]^{1 / 2}}\right|+ \\
& +\frac{(y-b)}{2} \ln \left|\frac{(x+a)+\left[(x+a)^{2}+(y-b)^{2}+z^{2}\right]^{1 / 2}}{(x+a)-\left[(x+a)+(y-b)^{2}+z^{2}\right]^{1 / 2}}\right|+ \\
& +z \operatorname{arctg} \frac{z\left[(x-a)^{2}+(y+b)^{2}+z^{2}\right]^{1 / 2}}{(x-a)(y+b)}+ \\
& +\frac{(x-a)}{2} \ln \left|\frac{(y+b)+\left[(x-a)^{2}+(y+b)^{2}+z^{2}\right]^{1 / 2}}{(y+b)-\left[(x-a)+(y+b)^{2}+z^{2}\right]^{1 / 2}}\right|+ \\
& +\frac{(y+b)}{2} \ln \left|\frac{(x-a)+\left[(x-a)^{2}+(y+b)^{2}+z^{2}\right]^{1 / 2}}{(x-a)-\left[(x-a)+(y+b)^{2}+z^{2}\right]^{1 / 2}}\right|- \\
& -z \operatorname{arctg} \frac{z\left[(x-a)^{2}+(y-b)^{2}+z^{2}\right]^{1 / 2}}{(x-a)(y-b)}-
\end{aligned}
$$




$$
\begin{aligned}
-\frac{(x-a)}{2} \ln \left|\frac{(y-b)+\left[(x-a)^{2}+(y-b)^{2}+z^{2}\right]^{1 / 2}}{(y-b)-\left[(x-a)+(y-b)^{2}+z^{2}\right]^{1 / 2}}\right|- \\
\left.-\frac{(y-b)}{2} \ln \left|\frac{(x-a)+\left[(x-a)^{2}+(y-b)^{2}+z^{2}\right]^{1 / 2}}{(x-a)-\left[(x-a)+(y-b)^{2}+z^{2}\right]^{1 / 2}}\right|\right\} .
\end{aligned}
$$

Предельный переход от (4.68) путем $b= \pm \infty$ дает функцию давления для задачи плоского деформирования полупространства равномерно распределенной нагрузкой интенсивностью $p\left(\mathrm{H} / \mathrm{M}^{2}\right)$ по бесконечной полосе шириной $2 a$ (рис. 4.7).

$$
\sigma=\frac{p}{\pi}\left[\operatorname{arctg} \frac{x+a}{z}-\operatorname{arctg} \frac{x-a}{z}\right] .
$$

Производные (4.71) будут:

$$
\begin{aligned}
& \frac{\partial}{\partial x} \frac{p}{\pi}\left(\operatorname{arctg} \frac{x+a}{z}-\operatorname{arctg} \frac{x-a}{z}\right)=-\frac{p}{\pi} \frac{4 a x z}{\left[\left(x^{2}+z^{2}-a^{2}\right)^{2}+4 a^{2} z^{2}\right]} \\
& \frac{\partial}{\partial z} \frac{p}{\pi}\left(\operatorname{arctg} \frac{x+a}{z}-\operatorname{arctg} \frac{x-a}{z}\right)=-\frac{p}{\pi} \frac{2 a\left(x^{2}-a^{2}-z^{2}\right)}{\left[\left(x^{2}+z^{2}-a^{2}\right)^{2}+4 a^{2} z^{2}\right]} .
\end{aligned}
$$

Окончательно компоненты напряженного состояния при плоском деформировании полупространства равномерно распределенной нагрузкой по бесконечной полосе шириной $2 a$ будут иметь вид:

$$
\sigma_{x}=\frac{p}{\pi}\left\{\left(\operatorname{arctg} \frac{x+a}{z}-\operatorname{arctg} \frac{x-a}{z}\right)-\frac{2 a\left(x^{2}-a^{2}-z^{2}\right)}{\left[\left(x^{2}+z^{2}-a^{2}\right)^{2}+4 a^{2} z^{2}\right]}\right\}
$$




$$
\begin{gathered}
\sigma_{z}=\frac{p}{\pi}\left\{\left(\operatorname{arctg} \frac{x+a}{z}-\operatorname{arctg} \frac{x-a}{z}\right)+\frac{2 a\left(x^{2}-a^{2}-z^{2}\right)}{\left[\left(x^{2}+z^{2}-a^{2}\right)^{2}+4 a^{2} z^{2}\right]}\right\} \\
\sigma_{y}=\sigma=\frac{p}{\pi}\left(\operatorname{arctg} \frac{x+a}{z}-\operatorname{arctg} \frac{x-a}{z}\right) \\
\tau_{x z}=\frac{p}{\pi} \frac{4 a x z^{2}}{\left[\left(x^{2}+z^{2}-a^{2}\right)^{2}+4 a^{2} z^{2}\right]}
\end{gathered}
$$

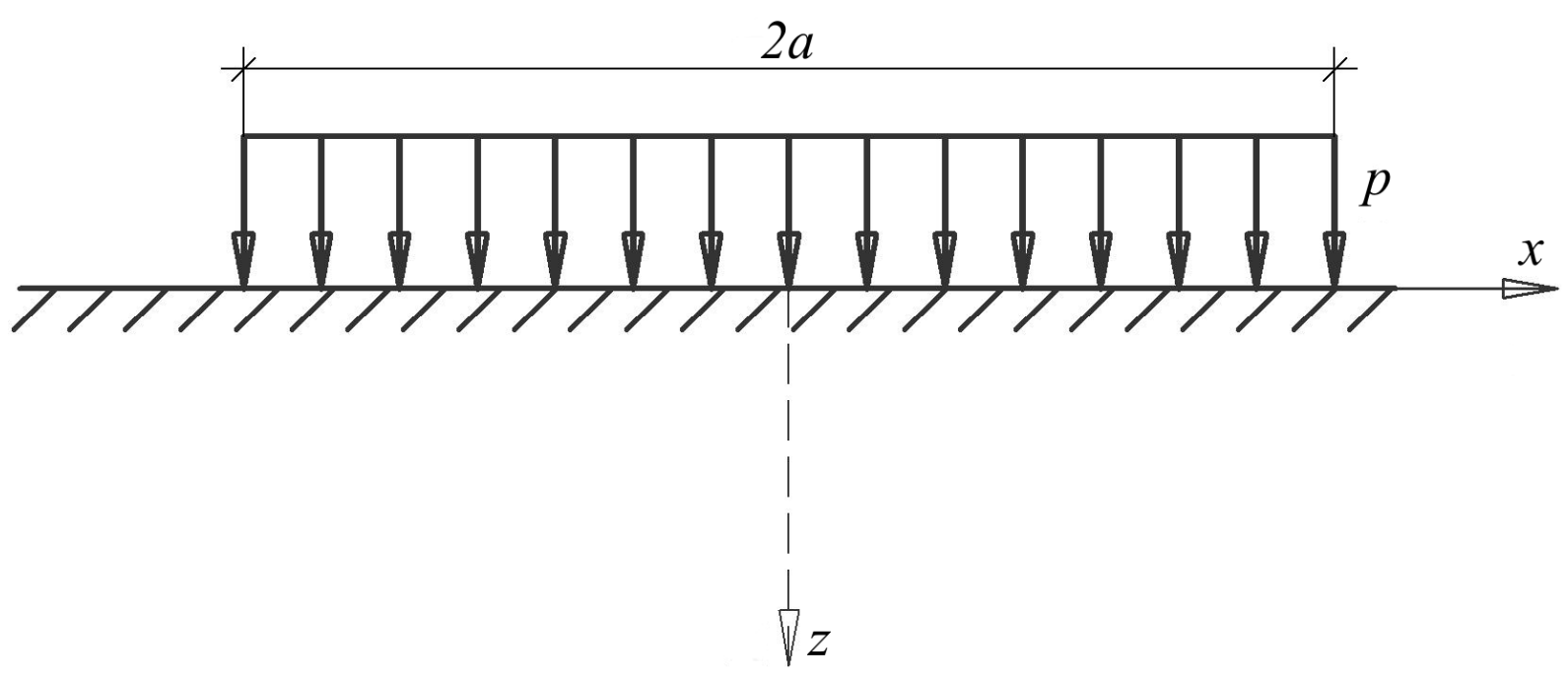

Рис. 4.7. Схема полупространства, равномерно нагруженного полосовой нагрузкой

Компоненты сдвиговых смещений согласно (3.28)-(3.30) будут:

$$
\begin{aligned}
& U^{\mathrm{c}}=-K^{\mathrm{c}} \frac{\partial \sigma}{\partial x}=K^{\mathrm{c}} \frac{p}{\pi} \frac{4 a x z}{\left[\left(x^{2}+z^{2}-a^{2}\right)^{2}+4 a^{2} z^{2}\right]} \\
& W^{\mathrm{c}}=-K^{\mathrm{c}} \frac{\partial \sigma}{\partial z}=K^{\mathrm{c}} \frac{p}{\pi} \frac{2 a\left(x^{2}-a^{2}-z^{2}\right)}{\left[\left(x^{2}+z^{2}-a^{2}\right)^{2}+4 a^{2} z^{2}\right]} .
\end{aligned}
$$


Перемещения, вызванные объемным деформированием, определяются интегрированием:

$$
\begin{gathered}
W^{0}=K^{0} \int_{\infty}^{z} \varepsilon_{z}^{0} d z \\
W^{0}=K^{0} \frac{p}{\pi}\left[\int_{z_{1}}^{z} \operatorname{arctg} \frac{x+a}{z} d z-\int_{z_{1}}^{z} \operatorname{arctg} \frac{x-a}{z} d z\right]= \\
=K^{0} \frac{p}{\pi}\left[\int_{z_{1}}^{z} \operatorname{arctg} \frac{z}{x+a} d z--\int_{z_{1}}^{z} \operatorname{arctg} \frac{z}{x-a} d z\right] .
\end{gathered}
$$

Согласно [23] эти интегралы равны:

$$
\begin{aligned}
W^{0}=K^{0} \frac{p}{\pi}\left\{z \operatorname{arcctg} \frac{z}{x+a}\right. & +\frac{x+a}{2} \ln \left|(x+a)^{2}+z^{2}\right|- \\
& \left.-z \operatorname{arcctg} \frac{z}{x-a}-\frac{x-a}{2} \ln \left|(x-a)^{2}+z^{2}\right|\right\}\left.\right|_{z_{1}} ^{z} .
\end{aligned}
$$

В выражении (4.72) нижний предел $z \rightarrow \infty$ брать не следует, поскольку осадка от изменения плотности среды будет неограниченно возрастать, поэтому должна определяться величина сжатия конкретного упругого слоя.

\subsection{6. Касательная нагрузка, равномерно распределенная на поверхности полупространства вдоль линий конечной и бесконечной длины}

Решение этой задачи можно получить соответствующим интегрированием компонентов решения (4.22)-(4.33). Схема нагружения полупространства приведена на рис. 4.8 . 
Функция давления может быть получена интегрированием (4.29):

$$
\sigma=-\frac{P x}{2 \pi} \int_{-b}^{b} \frac{d(y-\varepsilon)}{\left[x^{2}+(y-\varepsilon)^{2}+z^{2}\right]^{3 / 2}}=-\left.\frac{P x}{2 \pi\left(x^{2}+z^{2}\right)} \frac{(y-\varepsilon)}{\left[x^{2}+(y-\varepsilon)^{2}+z^{2}\right]^{3 / 2}}\right|_{-b} ^{b},
$$

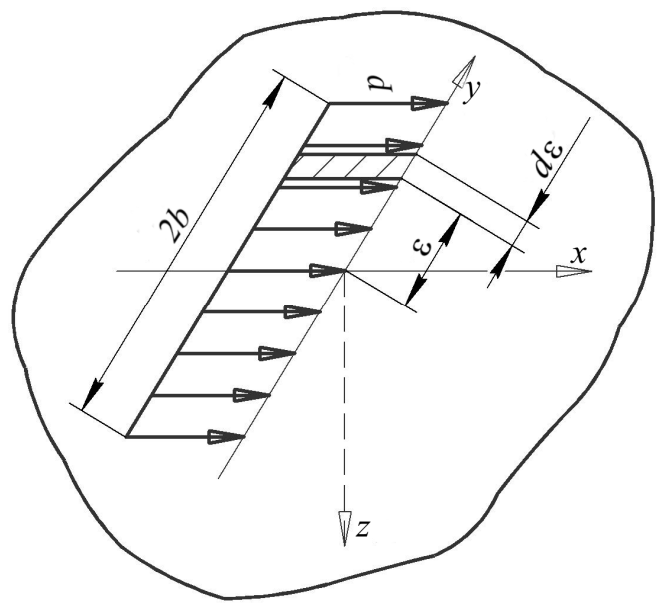

Рис. 4.8. Схема загружения поверхности полупространства касательной нагрузкой, равномерно распределенной вдоль линии длиной $2 b$

Компонент нормального напряжения $\sigma_{x}$ определяется интегрированием компонента (4.23):

$$
\begin{aligned}
\sigma_{x}=-\frac{3 P x^{3}}{2 \pi} \int_{-b}^{b} \frac{d(y-\varepsilon)}{\left[x^{2}+(y-\varepsilon)^{2}+z^{2}\right]^{3 / 2}}=-\frac{3 P x^{3}}{2 \pi\left(x^{2}+z^{2}\right)^{2}} \times \\
\\
\quad \times\left.\left\{\frac{(y-\varepsilon)}{\left[x^{2}+(y-\varepsilon)^{2}+z^{2}\right]^{1 / 2}}-\frac{(y-\varepsilon)^{3}}{3\left[x^{2}+(y-\varepsilon)^{2}+z^{2}\right]^{3 / 2}}\right\}\right|_{-b} ^{b},
\end{aligned}
$$




$$
\begin{gathered}
\sigma_{x}=-\frac{3 P x^{3}}{2 \pi\left(x^{2}+z^{2}\right)^{2}}\left\{\frac{(y+b)}{\left[x^{2}+(y+b)^{2}+z^{2}\right]^{1 / 2}}-\frac{(y+b)^{3}}{3\left[x^{2}+(y+b)^{2}+z^{2}\right]^{3 / 2}}-\right. \\
\left.-\frac{(y-b)}{\left[x^{2}+(y-b)^{2}+z^{2}\right]^{1 / 2}}-\frac{(y-b)^{3}}{3\left[x^{2}+(y-b)^{2}+z^{2}\right]^{1 / 2}}\right\} .
\end{gathered}
$$

Компонент нормального напряжения $\sigma_{y}$ определяется интегрированием компонента (4.24):

$$
\begin{gathered}
\sigma_{y}=-\frac{3 P x}{2 \pi} \int_{-b}^{b} \frac{d(y-\varepsilon)}{\left[x^{2}+(y-\varepsilon)^{2}+z^{2}\right]^{\frac{3}{2}}}=-\left.\frac{3 P x}{2 \pi\left(x^{2}+z^{2}\right)} \frac{(y-\varepsilon)^{3}}{\left[x^{2}+(y-\varepsilon)^{2}+z^{2}\right]^{\frac{3}{2}}}\right|_{-b} ^{b}, \\
\sigma_{y}=\frac{P x}{2 \pi\left(x^{2}+z^{2}\right)}-\left\{\frac{(y+b)^{3}}{\left[x^{2}+(y+b)^{2}+z^{2}\right]^{\frac{3}{2}}}-\frac{(y-b)^{3}}{\left[x^{2}+(y-b)^{2}+z^{2}\right]^{\frac{3}{2}}}\right\} . \text { (4.75) }
\end{gathered}
$$

Компонент нормального напряжения $\sigma_{z}$ определяется интегрированием компонента (4.25):

$$
\begin{aligned}
\sigma_{z}=-\frac{3 P x z^{2}}{2 \pi} \int_{-b}^{b} \frac{d(y-\varepsilon)}{\left[x^{2}-(y-\varepsilon)^{2}+z^{2}\right]^{5 / 2}}=-\frac{3 P x z^{2}}{2 \pi\left(x^{2}+z^{2}\right)^{2}} \times \\
\quad \times\left\{\frac{(y-\varepsilon)}{\left[x^{2}-(y-\varepsilon)^{2}+z^{2}\right]^{1 / 2}}-\left.\frac{1}{3} \frac{(y-\varepsilon)^{3}}{\left[x^{2}+(y-\varepsilon)^{2}+z^{2}\right]^{3 / 2}}\right|_{-b} ^{b} ;\right.
\end{aligned}
$$




$$
\begin{aligned}
\sigma_{z}=\frac{3 P x z^{2}}{2 \pi\left(x^{2}+z^{2}\right)^{2}} & \left\{\frac{(y+b)}{\left[x^{2}+(y+b)^{2}+z^{2}\right]^{1 / 2}}-\frac{(y+b)^{3}}{3\left[x^{2}+(y+b)^{2}+z^{2}\right]^{3 / 2}}-\right. \\
& \left.-\frac{(y-b)}{\left[x^{2}+(y-b)^{2}+z^{2}\right]^{1 / 2}}+\frac{(y-b)^{3}}{3\left[x^{2}+(y-b)^{2}+z^{2}\right]^{3 / 2}}\right\} .
\end{aligned}
$$

Компонент касательного напряжения $\tau_{x y}$ определяется интегрированием компонента (4.26):

$$
\begin{gathered}
\tau_{x y}=\frac{3 P x^{2}}{2 \pi} \int_{-b}^{b} \frac{(y-\varepsilon) d(y-\varepsilon)}{\left[x^{2}+(y-\varepsilon)^{2}+z^{2}\right]^{5 / 2}}=-\left.\frac{P x^{2}}{2 \pi} \frac{1}{\left[x^{2}+(y-\varepsilon)^{2}+z^{2}\right]^{3 / 2}}\right|_{-b} ^{b} \\
\tau_{x y}=\frac{P x^{2}}{2 \pi}\left\{\frac{1}{\left[x^{2}+(y+b)^{2}+z^{2}\right]^{3 / 2}}-\frac{1}{\left[x^{2}+(y-b)^{2}+z^{2}\right]^{3 / 2}}\right\} \cdot
\end{gathered}
$$

Компонент касательного напряжения $\tau_{y z}$ определяется интегрированием компонента (4.27):

$$
\begin{gathered}
\tau_{y z}=\frac{3 P x z}{2 \pi} \int_{-b}^{b} \frac{(y-\varepsilon) d(y-\varepsilon)}{\left[x^{2}+(y-\varepsilon)^{2}+z^{2}\right]^{5 / 2}}=-\left.\frac{3 P x z}{2 \pi}\left\{\frac{1}{3\left[x^{2}+(y-\varepsilon)^{2}+z^{2}\right]^{3 / 2}}\right\}\right|_{-b} ^{b} \\
\tau_{y z}=-\frac{P x z}{2 \pi}\left\{\frac{1}{\left[x^{2}+(y+b)^{2}+z^{2}\right]^{3 / 2}}-\frac{1}{\left[x^{2}+(y-b)^{2}+z^{2}\right]^{3 / 2}}\right\} \cdot(4.78)
\end{gathered}
$$

Компонент касательного напряжения $\tau_{z x}$ определяется интегрированием компонента (4.28): 


$$
\begin{aligned}
& \tau_{z x}=-\frac{3 P x^{2} z}{2 \pi} \int_{-b}^{b} \frac{d(y-\varepsilon)}{\left[x^{2}+(y-\varepsilon)^{2}+z^{2}\right]^{5 / 2}}=-\frac{3 P x^{2} z}{2 \pi\left(x^{2}+z^{2}\right)^{2}} \times \\
& \times\left\{\frac{(y-\varepsilon)}{\left[x^{2}+(y-\varepsilon)^{2}+z^{2}\right]^{1 / 2}}-\frac{(y-\varepsilon)^{3}}{3\left[x^{2}+(y-\varepsilon)^{2}+z^{2}\right]^{3 / 2}}\right\} \\
& \tau_{z x}=\frac{3 P x^{2} z}{2 \pi\left(x^{2}+z^{2}\right)^{2}}\left\{\frac{(y+b)}{\left[x^{2}+(y+b)^{2}+z^{2}\right]^{\frac{1}{2}}}-\frac{(y+b)^{3}}{3\left[x^{2}+(y+b)^{2}+z^{2}\right]^{\frac{3}{2}}}-\right. \\
& \left.-\frac{(y-b)}{\left[x^{2}+(y-b)^{2}+z^{2}\right]^{\frac{1}{2}}}+\frac{(y-b)^{3}}{3\left[x^{2}+(y-b)^{2}+z^{2}\right]^{\frac{3}{2}}}\right\}
\end{aligned}
$$

Сложив (4.74)-(4.76), можно убедиться, что функция давления (4.73) представляет среднее значение из трех нормальных напряжений $\sigma_{x}, \sigma_{y}, \sigma_{z}$.

По известной функции давления (4.73) определяются компоненты сдвиговых смещений в соответствии с (3.28)-(3.30):

$$
\begin{aligned}
& U^{\mathrm{c}}=-K^{\mathrm{c}} \frac{P}{2 \pi} \frac{\partial}{\partial x}\left\{\frac{x}{\left(x^{2}+z^{2}\right)}\left[\frac{(y+b)}{\left[x^{2}+(y+b)^{2}+z^{2}\right]^{\frac{1}{2}}}-\frac{(y-b)}{\left[x^{2}+(y-b)^{2}+z^{2}\right]^{\frac{1}{2}}}\right]\right\} \\
& U^{\mathrm{c}}=\frac{K^{\mathrm{c}} P}{2 \pi}\left\{\frac{x^{2}-z^{2}}{\left(x^{2}+z^{2}\right)^{2}}\left[\frac{(y+b)}{\left[x^{2}+(y+b)^{2}+z^{2}\right]^{1 / 2}}-\frac{(y-b)}{\left[x^{2}+(y-b)^{2}+z^{2}\right]^{1 / 2}}\right]+\right.
\end{aligned}
$$




$$
\begin{aligned}
& \left.+\frac{x^{2}}{x^{2}+z^{2}}\left[\frac{(y+b)}{\left[x^{2}+(y+b)^{2}+z^{2}\right]^{3 / 2}}-\frac{(y-b)}{\left[x^{2}+(y-b)^{2}+z^{2}\right]^{3 / 2}}\right]\right\} ; \text { (4.80) } \\
& V^{\mathrm{c}}=-K^{\mathrm{c}} \frac{P x}{2 \pi\left(x^{2}+z^{2}\right)} \frac{d}{d y}\left\{\frac{(y+b)}{\left[x^{2}+(y+b)^{2}+z^{2}\right]^{\frac{1}{2}}}-\frac{(y-b)}{\left[x^{2}+(y-b)^{2}+z^{2}\right]^{\frac{1}{2}}}\right\} \\
& V^{\mathrm{c}}=-K^{\mathrm{c}} \frac{P x}{2 \pi}\left[\frac{1}{\left[x^{2}+(y+b)^{2}+z^{2}\right]^{3 / 2}}-\frac{1}{\left[x^{2}+(y-b)^{2}+z^{2}\right]^{3 / 2}}\right] \\
& W^{\mathrm{c}}=-K^{\mathrm{c}} \frac{P}{2 \pi} \frac{d}{d z}\left\{\frac{x}{\left(x^{2}+z^{2}\right)}\left[\frac{(y+b)}{\left[x^{2}+(y+b)^{2}+z^{2}\right]^{\frac{1}{2}}}-\frac{(y-b)}{\left[x^{2}+(y-b)^{2}+z^{2}\right]^{\frac{1}{2}}}\right]\right\} \text {; } \\
& W^{c}=K^{c} \frac{P}{2 \pi} \frac{x z}{\left(x^{2}+z^{2}\right)}\left\{\frac{2}{\left(x^{2}+z^{2}\right)} \times\right. \\
& \times\left[\frac{(y+b)}{\left[x^{2}+(y+b)^{2}+z^{2}\right]^{\frac{1}{2}}}-\frac{(y-b)}{\left[x^{2}+(y-b)^{2}+z^{2}\right]^{\frac{1}{2}}}\right]+ \\
& \left.+\left[\frac{(y+b)}{\left[x^{2}+(y+b)^{2}+z^{2}\right]^{3 / 2}}-\frac{(y-b)}{\left[x^{2}+(y-b)^{2}+z^{2}\right]^{3 / 2}}\right]\right\} \text {. }
\end{aligned}
$$

Горизонтальные перемещения точек среды от объемного деформирования в соответствии с (3.19) будут направлены параллельно 
действию внешней нагрузки, т. е. параллельно оси $x$, они определяются интегрированием функции давления (4.73):

$$
\begin{gathered}
U^{0}=\int_{x}^{\infty} \varepsilon_{x} d x=K^{0} \int_{x}^{\infty} \sigma d x \\
U^{0}=K^{0} \int_{x}^{\infty} \frac{P x}{2 \pi\left(x^{2}+z^{2}\right)}\left\{\frac{(y+b)}{\left[x^{2}+(y+b)^{2}+z^{2}\right]^{1 / 2}}-\frac{(y-b)}{\left[x^{2}+(y-b)^{2}+z^{2}\right]^{1 / 2}}\right\} d x .
\end{gathered}
$$

Постольку подобный интеграл определялся ранее (4.59), запишем его, поменяв местами координаты $x$ и $z$ :

$$
\int_{x}^{\infty} \frac{x d x}{\left(x^{2}+z^{2}\right)\left(x^{2}+y^{2}+z^{2}\right)^{1 / 2}}=\frac{1}{2 y} \ln \left|\frac{y+\left(x^{2}+y^{2}+z^{2}\right)^{1 / 2}}{y-\left(x^{2}+y^{2}+z^{2}\right)^{1 / 2}}\right|_{x}^{\infty} .
$$

После соответствующей подстановки в (4.83) окончательно горизонтальные перемещения от изменения объема среды будут определяться выражением

$$
\begin{aligned}
U^{0}=K^{0} \frac{P}{4 \pi}\left\{\frac{1}{(y+b)} \ln \left|\frac{(y+b)+\left[x^{2}+(y+b)^{2}+z^{2}\right]^{1 / 2}}{(y+b)-\left[x^{2}+(y+b)^{2}+z^{2}\right]^{1 / 2}}\right|-\right. \\
\\
\left.-\frac{1}{y-b} \ln \left|\frac{(y-b)+\left[x^{2}+(y-b)^{2}+z^{2}\right]^{1 / 2}}{(y-b)-\left[x^{2}+(y-b)^{2}+z^{2}\right]^{1 / 2}}\right|\right\} .
\end{aligned}
$$

При увеличении длины линии загружения от $-\infty$ до $+\infty$ (см. рис. 4.8) получим плоское деформирование полупространства касательной нагрузкой, равномерно распределенной вдоль бесконечной линии. На рис. 4.9 приведена схема сечения полупространства плоскостью $x o z$, нормальной к линии распределения внешней нагрузки. 
Напряженное состояние полупространства для рассматриваемого случая можно получить путем предельного перехода от (4.73)-(4.79), в результате чего имеем:

функция давления

$$
\sigma=\frac{P x}{\pi\left(x^{2}+z^{2}\right)}
$$

компоненты напряжений

$$
\left.\begin{array}{l}
\sigma_{x}=\frac{2 P x^{3}}{\pi\left(x^{2}+z^{2}\right)^{2}} ; \quad \sigma_{y}=\frac{P x}{\pi\left(x^{2}+z^{2}\right)} ; \\
\sigma_{z}=\frac{2 P x z^{2}}{\pi\left(x^{2}+z^{2}\right)^{2}} ; \\
\tau_{x y}=\tau_{y z}=0 ; \quad \tau_{z x}=\frac{2 P x^{2} z}{\pi\left(x^{2}+z^{2}\right)^{2}} .
\end{array}\right\}
$$

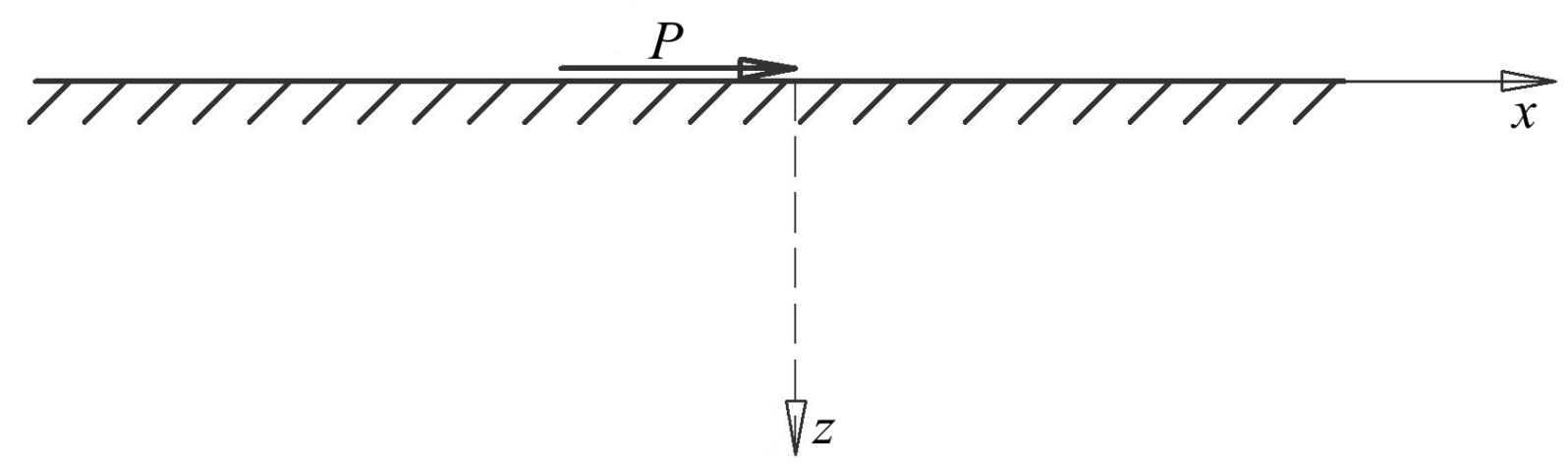

Рис. 4.9. Схема плоского деформирования полупространства касательной нагрузкой

Компоненты смещений от сдвигового деформирования согласно (3.28)-(3.30) будут

$$
U^{\mathrm{c}}=-K^{\mathrm{c}} \frac{P}{\pi} \frac{d}{d x}\left[\frac{x}{\left(x^{2}+z^{2}\right)}\right]=-K^{\mathrm{c}} \frac{P}{\pi} \frac{x^{2}+z^{2}-2 x^{2}}{\left(x^{2}+z^{2}\right)^{2}},
$$




$$
\begin{gathered}
U^{\mathrm{c}}=K^{\mathrm{c}} \frac{P}{\pi} \frac{x^{2}-z^{2}}{\left(x^{2}+z^{2}\right)^{2}} ; \\
W^{\mathrm{c}}=-K^{\mathrm{c}} \frac{P}{\pi} \frac{d}{d z}\left[\frac{x}{\left(x^{2}+z^{2}\right)}\right]=-K^{\mathrm{c}} \frac{P}{\pi} \frac{(-2 x z)}{\left(x^{2}+z^{2}\right)^{2}}, \\
W^{\mathrm{c}}=K^{\mathrm{c}} \frac{2 P}{\pi} \frac{x z}{\left(x^{2}+z^{2}\right)^{2}} .
\end{gathered}
$$

Перемещения от изменения объема среды получим в соответствии c (3.17)-(3.19):

$$
\begin{gathered}
U^{0}=K^{0} \frac{P}{\pi} \int_{x_{1}}^{x_{2}} \frac{x d x}{z^{2}+x^{2}}=\left.K^{0} \frac{P}{2 \pi} \ln \left(z^{2}+x^{2}\right)\right|_{x_{1}} ^{x_{2}}, \\
U^{0}=K^{0} \frac{P}{2 \pi} \ln \frac{z^{2}+x_{2}^{2}}{z^{2}+x_{1}^{2}} .
\end{gathered}
$$

Поскольку при $x \rightarrow \infty$ смещения $U^{0}$ неограниченно возрастают, то по этой формуле следует определять абсолютную деформацию конкретного, конечного участка - от $x_{1}$ до $x_{2}$.

\subsection{7. Наклонная нагрузка, равномерно распределенная на поверхности полупространства вдоль линий конечной и бесконечной длины}

В том случае, когда равномерно распределенная нагрузка интенсивностью $P(\mathrm{H} / \mathrm{M})$ вдоль линии конечной длины действует наклонно к поверхности полупространства, составляя с осями координат $x$ и $z$ углы соответственно $\alpha$ и $\gamma$ (рис. 4.10), ее следует разложить на две составляющие по координатным осям соответственно: $P_{x}=P \cos \alpha$, $P_{z}=P \cos \gamma$, и составить комбинацию решений для нагрузки, распределенной по линии конечной длины, - решения (4.47)-(4.55), (4.58) 
для вертикальной составляющей $P_{z}$ и решения (4.73)-(4.82), (4.84) для горизонтальной составляющей $P_{x}$. Приведем здесь это решение без компонентов напряжений, которые, в общем, не играют никакой роли при определении абсолютных и относительных деформаций.

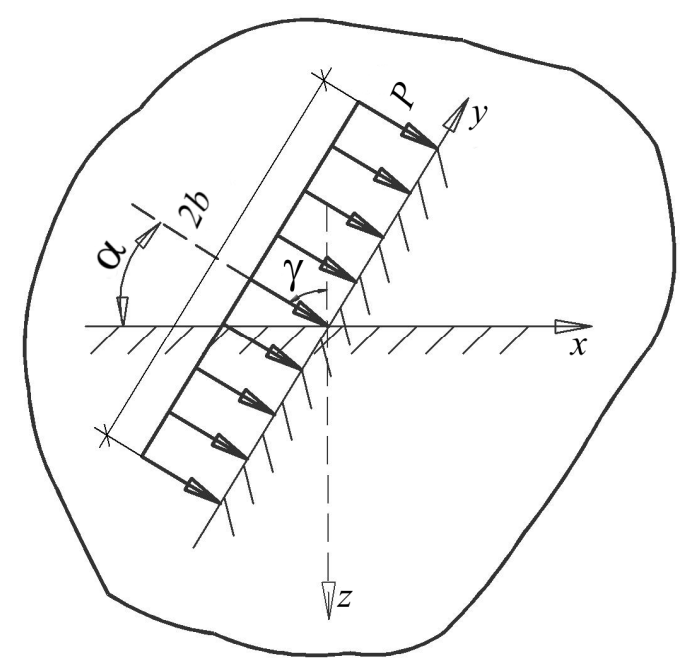

Рис. 4.10. Схема загружения полупространства наклонной равномерно распределенной вдоль линии нагрузкой

Функция давления

$\sigma=\frac{P \cos \gamma}{2 \pi} \frac{z}{\left(x^{2}+z^{2}\right)}\left\{\frac{(y+b)}{\left[x^{2}+(y+b)^{2}+z^{2}\right]^{1 / 2}}-\frac{y-b}{\left[x^{2}+(y-b)^{2}+z^{2}\right]^{1 / 2}}\right\}+$
$+\frac{P \cos \alpha}{2 \pi} \frac{x}{\left(x^{2}+z^{2}\right)}\left\{\frac{(y+b)}{\left[x^{2}+(y+b)^{2}+z^{2}\right]^{1 / 2}}-\frac{(y-b)}{\left[x^{2}+(y-b)^{2}+z^{2}\right]^{1 / 2}}\right\}$.

Компоненты смещений от чистого сдвига

$U^{\mathrm{c}}=-K^{\mathrm{c}} \frac{\partial \sigma}{\partial x}=K^{\mathrm{c}} \frac{P \cos \gamma \cdot x z}{2 \pi\left(x^{2}+z^{2}\right)} \times$ 


$$
\begin{aligned}
& \times\left\{\frac{2}{\left(x^{2}+z^{2}\right)}\left[\frac{(y+b)}{\left[x^{2}+(y+b)^{2}+z^{2}\right]^{1 / 2}}-\frac{y-b}{\left[x^{2}+(y-b)^{2}+z^{2}\right]^{1 / 2}}\right]+\right. \\
& \left.+\left[\frac{y+b}{\left[x^{2}+(y+b)^{2}+z^{2}\right]^{3 / 2}}-\frac{y-b}{\left[x^{2}+(y-b)^{2}+z^{2}\right]^{3 / 2}}\right]\right\}+K^{\mathrm{c}} \frac{P \cos \alpha}{2 \pi} \times \\
& \times\left\{\frac{x^{2}-z^{2}}{\left(x^{2}+z^{2}\right)^{2}}\left[\frac{y+b}{\left[x^{2}+(y+b)^{2}+z^{2}\right]^{1 / 2}}-\frac{y-b}{\left[x^{2}+(y-b)^{2}+z^{2}\right]^{1 / 2}}\right]+\right. \\
& \left.+\frac{x^{2}}{\left(x^{2}+z^{2}\right)}\left[\frac{y+b}{\left[x^{2}+(y+b)^{2}+z^{2}\right]^{3 / 2}}-\frac{y-b}{\left[x^{2}+(y-b)^{2}+z^{2}\right]^{3 / 2}}\right]\right\} \\
& V^{\mathrm{c}}=-K^{\mathrm{c}} \frac{\partial \sigma}{\partial y}=-K^{\mathrm{c}} \frac{P \cos \gamma(z)}{2 \pi} \times \\
& \times\left\{\frac{1}{\left[x^{2}+(y+b)^{2}+z^{2}\right]^{3 / 2}}-\frac{1}{\left[x^{2}+(y-b)^{2}+z^{2}\right]^{3 / 2}}\right\}- \\
& -K^{\mathrm{c}} \frac{P \cos \alpha(x)}{2 \pi}\left\{\frac{1}{\left[x^{2}+(y+b)^{2}+z^{2}\right]^{3 / 2}}-\frac{1}{\left[x^{2}+(y-b)^{2}+z^{2}\right]^{3 / 2}}\right\} \\
& W^{\mathrm{c}}=-K^{\mathrm{c}} \frac{\partial \sigma}{\partial z}=K^{\mathrm{c}} \frac{P \cos \gamma}{2 \pi\left(x^{2}+z^{2}\right)} \times
\end{aligned}
$$




$$
\begin{aligned}
& \times\left\{\frac{z^{2}-x^{2}}{\left(x^{2}+z^{2}\right)}\left[\frac{(y+b)}{\left[x^{2}+(y+b)^{2}+z^{2}\right]^{1 / 2}}-\frac{(y-b)}{\left[x^{2}+(y-b)^{2}+z^{2}\right]^{1 / 2}}\right]+z^{2} \times\right. \\
& \left.\times\left[\frac{(y+b)}{\left[x^{2}+(y+b)^{2}+z^{2}\right]^{\frac{3}{2}}}-\frac{(y-b)}{\left[x^{2}+(y-b)^{2}+z^{2}\right]^{\frac{3}{2}}}\right]\right\}+K^{\mathrm{c}} \frac{P \cos \alpha}{2 \pi} \frac{x z}{\left(x^{2}+z^{2}\right)} \times \\
& \times\left\{\frac{(y+b)}{\left(x^{2}+z^{2}\right)}\left[\frac{(y-b)}{\left[x^{2}+(y+b)^{2}+z^{2}\right]^{1 / 2}}-\frac{\left[x^{2}+(y-b)^{2}+z^{2}\right]^{1 / 2}}{\left[x^{2}\right.}\right]+\right. \\
& \left.+\left[\frac{(y+b)}{\left[x^{2}+(y+b)^{2}+z^{2}\right]^{3 / 2}}-\frac{(y-b)}{\left[x^{2}+(y-b)^{2}+z^{2}\right]^{3 / 2}}\right]\right\} .
\end{aligned}
$$

Компоненты смещений от объемного деформирования

$$
\begin{gathered}
U^{0}=K^{0} \frac{P \cos \alpha}{4 \pi}\left\{\frac{1}{(y+b)} \ln \left|\frac{(y+b)+\left[x^{2}+(y+b)^{2}+z^{2}\right]^{1 / 2}}{(y+b)-\left[x^{2}+(y+b)^{2}+z^{2}\right]^{1 / 2}}\right|-\right. \\
\left.-\frac{1}{(y-b)} \ln \left|\frac{(y-b)+\left[x^{2}+(y-b)^{2}+z^{2}\right]^{1 / 2}}{(y-b)-\left[x^{2}+(y-b)^{2}+z^{2}\right]^{1 / 2}}\right|\right\} ; \\
W^{0}=K^{0} \frac{P \cos \gamma}{4 \pi}\left\{\frac{1}{(y+b)} \ln \left|\frac{(y+b)+\left[x^{2}+(y+b)^{2}+z^{2}\right]^{1 / 2}}{(y+b)-\left[x^{2}+(y+b)^{2}+z^{2}\right]^{1 / 2}}\right|-\right.
\end{gathered}
$$




$$
\left.-\frac{1}{(y-b)} \ln \left|\frac{(y-b)+\left[x^{2}+(y-b)^{2}+z^{2}\right]^{1 / 2}}{(y-b)-\left[x^{2}+(y-b)^{2}+z^{2}\right]^{1 / 2}}\right|\right\} \text {. }
$$

Для случая действия на поверхности полупространства наклонной силы, равномерно распределенной вдоль бесконечной линии, мы получим плоское его деформирование.

Соответствующая схема может быть представлена рис. 4.10, на котором следует продлить воображаемую линию распределенной силы в обе стороны до бесконечности: $-\infty \leq b \leq+\infty$.

Разложив наклонную силу на вертикальную и горизонтальную составляющие, мы получим две задачи плоского деформирования полупространства от линейно распределенных сил, для каждой из которых имеется свое решение: (4.60)-(4.66) для $P_{z}$ и (4.85)-(4.89) для $P_{x}$. Общее решение рассматриваемой задачи будет представлять сумму этих решений. Запишем его без компонентов напряжений, подобно тому, как это было выполнено в предыдущем решении, когда линия распределения наклонной силы имела ограниченную длину:

$$
\begin{gathered}
\sigma=\frac{P}{\pi\left(x^{2}+z^{2}\right)}(z \cos \gamma+x \cos \alpha) \\
U^{\mathrm{c}}=\frac{K^{\mathrm{c} P}}{\pi\left(x^{2}+z^{2}\right)^{2}}\left[2 x z \cos \gamma+\left(x^{2}-z^{2}\right) \cos \alpha\right] \\
W^{\mathrm{c}}=-\frac{K^{\mathrm{c} P}}{\pi\left(x^{2}+z^{2}\right)^{2}}\left[\left(x^{2}-z^{2}\right) \cos \alpha-2 x z \cos \gamma\right] ; \\
U^{0}=K^{0} \frac{P \cos \alpha}{2 \pi} \ln \frac{z^{2}+x_{2}^{2}}{z^{2}+x_{1}^{2}} ; \\
W^{0}=K^{0} \frac{P \cos \gamma}{2 \pi} \ln \frac{x^{2}+z_{2}^{2}}{x^{2}+z_{1}^{2}} .
\end{gathered}
$$




\section{2. Напряженно-деформированное состояние упругого изотропного пространства от действия сосредоточенной внутри его силы}

Выше было показано, что напряженное состояние упругого полупространства от действия любой сосредоточенной на его поверхности силы и упругого пространства от сосредоточенной внутри его силы имеет радиальное распределение. Для упрощения пояснений будем сравнивать полупространство, на поверхности которого действует нормальная сила (рис. 4.11), и пространство с таким же расположением координат и направлением действия силы, приложенной внутри (рис. 4.12).

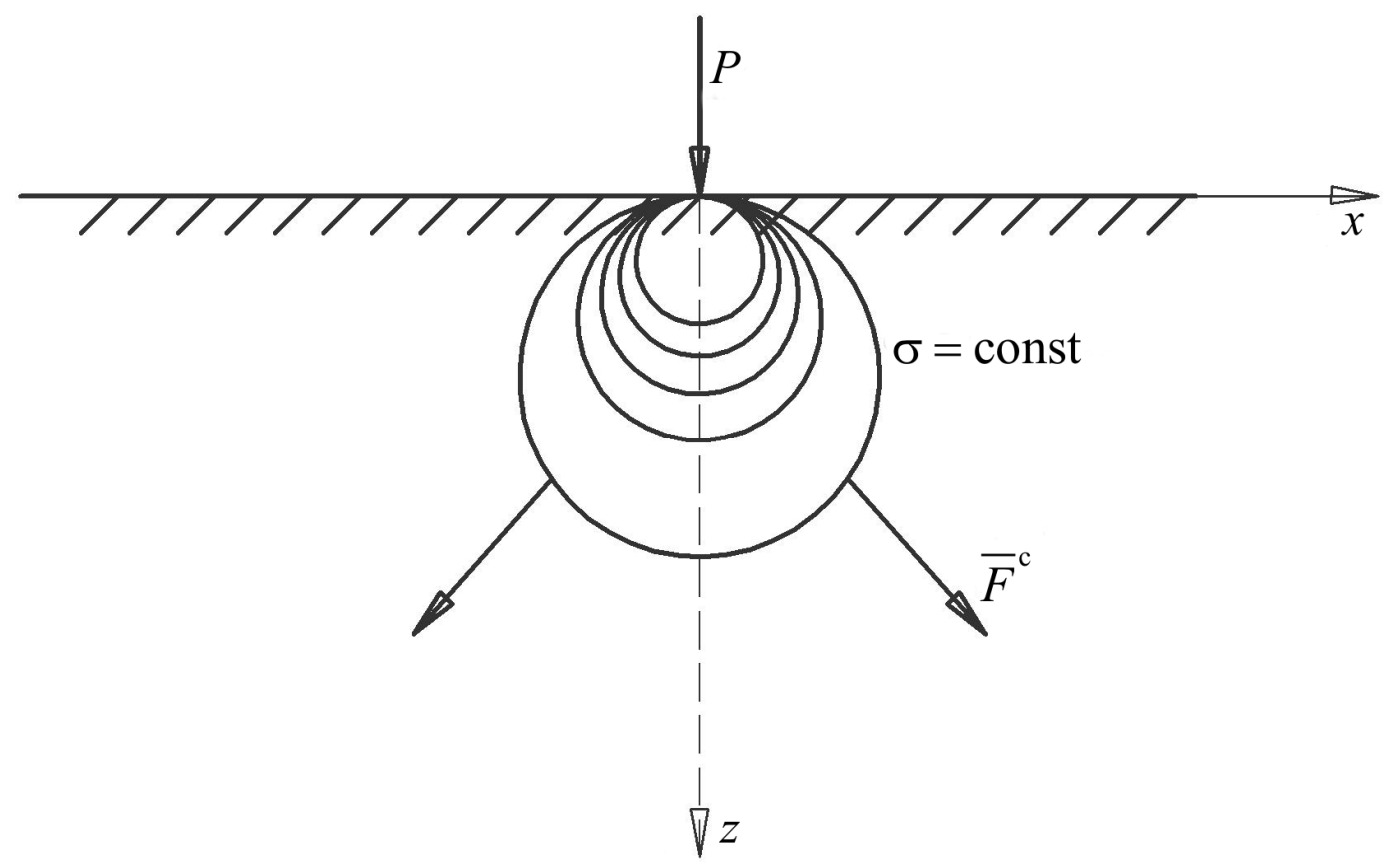

Рис. 4.11. Распределение поверхностей равного давления

в упругом полупространстве от действующей по нормали к нему силы

Очевидно, что в первом случае координата $z$ будет иметь только положительные значения, а во втором ее следует продлить и в отрицательную область. Поэтому в первом случае семейство поверхностей равного давления будет представлено сферами, центры которых находятся на оси $z$ и касаются ограничивающей плоскости под приложенной силой. С увеличением радиуса сферы уменьшается 
давление, и в пределе поверхностью нулевого давления будет ограничивающая плоскость $(z=0)$.

Во втором же случае, выше плоскости хоу, с изменением знака координаты $z$ давление также поменяет знак, и таким образом, в пространстве будет два симметрических семейства поверхностей равных давлений: одно с положительными давлениями (в положительной области координаты $z$ ), второе - с отрицательными давлениями (в отрицательной области координаты $z$ ). В соответствии с тем, что сдвиговые смещения направлены в сторону убывания градиентов давлений, симметрично расположенные точки упругого пространства будут смещаться в положительной области в сторону внешней нормали к поверхностям равных давлений, в отрицательной области в сторону внутренней нормали (см. рис. 4.12).

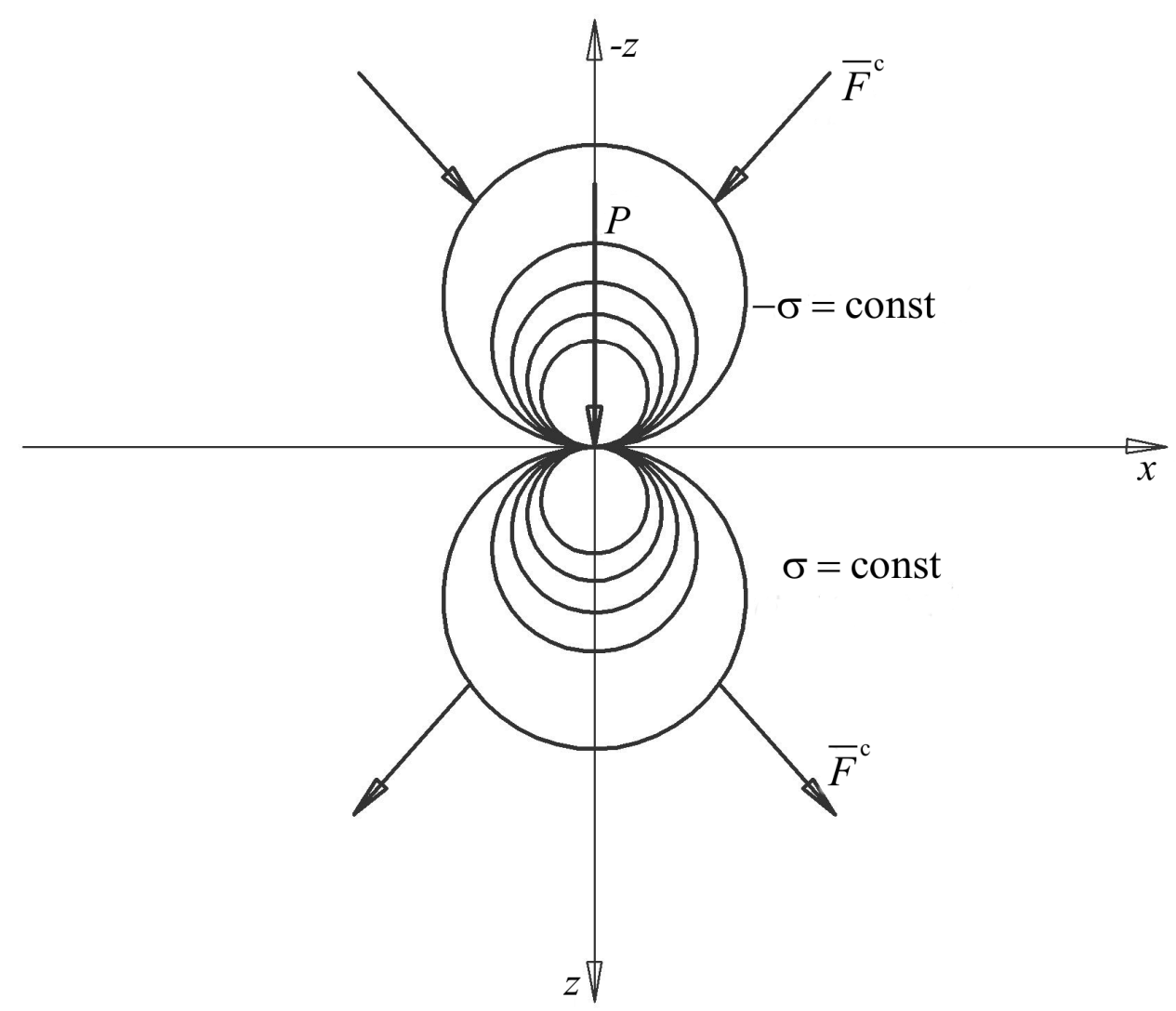

Рис. 4.12. Распределение поверхностей равного давления в упругом пространстве от сосредоточенной внутри его силы

Следует заметить, что в начале главы 2 нами была сделана оговорка о том, что в отличие от общепринятого в теории упругости знака сжимающего напряжения с минусом, в данной роботе было принято 
его значение с плюсом. В противном случае направление сдвигового смещения следовало бы брать отличным от принятого в векторном анализе.

После предварительных пояснений представим полное решение данной задачи. Так как компоненты напряжений в этой задаче приведены в системе (2.86), то воспользуемся ими для определения функции давления по зависимостям (3.28)-(3.30):

$$
\sigma=\frac{1}{3}\left(\sigma_{x}+\sigma_{y}+\sigma_{z}\right)=\frac{P}{4 \pi} \frac{z}{\left(x^{2}+y^{2}+z^{2}\right)^{3 / 2}} .
$$

С помощью функции давления (4.90) определяются компоненты сдвиговых смещений пространства:

$$
\begin{gathered}
U^{\mathrm{c}}=-K^{\mathrm{c}} \frac{P}{4 \pi} \frac{\partial}{\partial x}\left[\frac{z}{\left(x^{2}+y^{2}+z^{2}\right)^{3 / 2}}\right], \\
U^{\mathrm{c}}=K^{\mathrm{c}} \frac{3 P}{4 \pi} \frac{x z}{\left(x^{2}+y^{2}+z^{2}\right)^{5 / 2}}=K^{\mathrm{c}} \frac{3 P}{2 \pi} \frac{x z}{R^{5}} \\
V^{\mathrm{c}}=-K^{\mathrm{c}} \frac{3 P}{4 \pi} \frac{\partial}{\partial y}\left[\frac{z}{\left(x^{2}+y^{2}+z^{2}\right)^{3 / 2}}\right], \\
V^{\mathrm{c}}=K^{\mathrm{c}} \frac{3 P}{4 \pi} \frac{y z}{\left(x^{2}+y^{2}+z^{2}\right)^{5 / 2}}=K^{\mathrm{c}} \frac{3 P y z}{2 \pi R^{5}} . \\
W^{\mathrm{c}}=-K^{\mathrm{c}} \frac{3 P}{4 \pi} \frac{\partial}{\partial z}\left[\frac{z}{\left(x^{2}+y^{2}+z^{2}\right)}\right], \\
W^{\mathrm{c}}=-K^{\mathrm{c}} \frac{P}{4 \pi} \frac{2 z^{2}}{\left(x^{2}+y^{2}+z^{2}\right)^{5 / 2}=K^{\mathrm{c}} \frac{P\left(3 z^{2}-R^{2}\right)}{4 \pi R^{5}} .} .
\end{gathered}
$$


Компоненты линейных относительных деформаций от чистого сдвига определяются по зависимостям (3.31)-(3.33):

$$
\begin{gathered}
\varepsilon_{x}^{\mathrm{c}}=\frac{\partial U^{\mathrm{c}}}{\partial x}=K^{\mathrm{c}} \frac{3 P}{4 \pi} \frac{\partial}{\partial x}\left[\frac{x z}{\left(x^{2}+y^{2}+z^{2}\right)^{5 / 2}}\right]=K^{\mathrm{c}} \frac{3 P}{4 \pi} \frac{z\left(y^{2}+z^{2}-4 x^{2}\right)}{\left(x^{2}+y^{2}+z^{2}\right)^{7 / 2}}, \\
\varepsilon_{x}^{\mathrm{c}}=K^{\mathrm{c}} \frac{3 P}{4 \pi} \frac{z\left(R^{2}-5 x^{2}\right)}{R^{7}} ; \\
\varepsilon_{y}^{\mathrm{c}}=\frac{\partial V^{\mathrm{c}}}{\partial y}=K^{\mathrm{c}} \frac{3 P}{4 \pi} \frac{\partial}{\partial x}\left[\frac{y z}{\left(x^{2}+y^{2}+z^{2}\right)^{5 / 2}}\right]=K^{\mathrm{c}} \frac{3 P}{4 \pi} \frac{z\left(x^{2}+z^{2}-4 y^{2}\right)}{\left(x^{2}+y^{2}+z^{2}\right)^{7 / 2}}, \\
\varepsilon_{z}^{\mathrm{c}}=\frac{\partial W^{\mathrm{c}}}{\partial z}=K^{\mathrm{c}} \frac{P}{4 \pi} \frac{\partial}{\partial x}\left[\frac{2 z^{2}-x^{2}-y^{2}}{\left(x^{2}+y^{2}+z^{2}\right)^{5 / 2}}\right]=\frac{P}{4 \pi} \frac{z\left(9 x^{2}+9 y^{2}-6 z^{2}\right)}{\left(x^{2}+y^{2}+z^{2}\right)^{7 / 2}}, \\
\left.\varepsilon_{z}^{\mathrm{c}}=\frac{3 P}{4 \pi} \frac{z\left(R^{2}-5 y^{2}\right)}{R^{7}} ; \frac{R^{7}}{R^{7}} \cdot 5 z^{2}\right)
\end{gathered}
$$

Компоненты угловых деформаций от чистого сдвига определяются по зависимостям (3.34)-(3.36):

$$
\begin{gathered}
\gamma_{x y}^{\mathrm{c}}=-K^{\mathrm{c}} 2 \frac{\partial^{2} \sigma}{\partial x \partial y}=-K^{\mathrm{c}} \frac{P}{2 \pi} \frac{\partial^{2}}{\partial x \partial y}\left[\frac{z}{\left(x^{2}+y^{2}+z^{2}\right)^{3 / 2}}\right], \\
\gamma_{x y}^{\mathrm{c}}=-\frac{15 P}{2 \pi} \frac{x y z}{R^{7}}
\end{gathered}
$$




$$
\begin{gathered}
\gamma_{y z}^{\mathrm{c}}=-K^{\mathrm{c}} 2 \frac{\partial^{2} \sigma}{\partial y \partial z}=K^{\mathrm{c}} \frac{3 P}{2 \pi} \frac{y\left(x^{2}+y^{2}-4 z^{2}\right)}{\left(x^{2}+y^{2}+z^{2}\right)^{7 / 2}}, \\
\gamma_{y z}^{\mathrm{c}}=K^{\mathrm{c}} \frac{3 P}{2 \pi} \frac{y\left(R^{2}-5 z^{2}\right)}{R^{7}} ; \\
\gamma_{z x}^{\mathrm{c}}=-K^{\mathrm{c}} 2 \frac{\partial^{2} \sigma}{\partial y \partial z}=K^{\mathrm{c}} \frac{3 P}{2 \pi} \frac{x\left(x^{2}+y^{2}-4 z^{2}\right)}{\left(x^{2}+y^{2}+z^{2}\right)^{7 / 2}}, \\
\gamma_{z x}^{\mathrm{c}}=K^{\mathrm{c}} \frac{3 P}{2 \pi} \frac{x\left(R^{2}-5 z^{2}\right)}{R^{7}} .
\end{gathered}
$$

Жесткие повороты элементарного куба от чистого сдвига отсутствуют, т. е.

$$
\omega_{x}^{\mathrm{c}}=\omega_{y}^{\mathrm{c}}=\omega_{z}^{\mathrm{c}}=0
$$

Объемная относительная деформация в соответствии с (3.17) описывается зависимостью

$$
\theta=K^{0} \sigma=K^{0} \frac{P}{2 \pi} \frac{z}{R^{3}}
$$

Линейные относительные деформации от изменения объема в соответствии с (3.19) будут

$$
\begin{gathered}
\varepsilon_{x}^{0}=\varepsilon_{y}^{0}=0, \\
\varepsilon_{z}^{0}=K^{0} \frac{P}{2 \pi} \frac{z}{R^{3}},
\end{gathered}
$$

то есть смещения точек пространства от изменения объема среды будут происходить только по вертикали, они определяются интегрированием (4.91): 


$$
\begin{gathered}
W^{0}=\int_{z}^{\infty} \varepsilon_{z}^{0} d z=K^{0} \frac{P}{4 \pi} \int_{z}^{\infty} \frac{z d z}{\left(x^{2}+y^{2}+z^{2}\right)^{3 / 2}}-\left.K^{0} \frac{P}{4 \pi} \frac{1}{\left(x^{2}+y^{2}+z^{2}\right)^{1 / 2}}\right|_{x_{1}} ^{x_{2}}, \\
W^{0}=K^{0} \frac{P}{4 \pi R} .
\end{gathered}
$$

Следует заметить, что в верхней половине пространства (см. рис. 4.12) будет происходить расширение среды, в нижней - сжатие, а перемещения в обеих половинах пространства будут направлены в одну сторону, совпадающую с направлением приложенной силы $P$ при этом в точках, симметрично расположенных относительно координатной плоскости $x о y$, они будут иметь одинаковую величину.

По известному полю перемещений от изменения объема $W^{0}$ определяются соответствующие угловые относительные деформации согласно (1.9):

$$
\begin{gathered}
\gamma_{x y}^{0}=\frac{\partial U^{0}}{\partial y}+\frac{\partial V}{\partial x}=0 \\
\gamma_{y z}^{0}=\frac{\partial V^{0}}{\partial z}+\frac{\partial W^{0}}{\partial y}=0+K^{0} \frac{P}{4 \pi} \frac{\partial}{\partial y}\left[\frac{1}{\left(x^{2}+y^{2}+z^{2}\right)^{1 / 2}}\right], \\
\gamma_{z x}^{0}=\frac{\partial W^{0}}{\partial x}+\frac{\partial U^{0}}{\partial z}=K^{0} \frac{P}{4 \pi} \frac{P}{4 \pi} \frac{\partial}{\partial y}\left[\frac{1}{\left(x^{2}+y^{2}+z^{2}\right)^{1 / 2}}\right] \\
\gamma_{z x}^{0}=-K^{0} \frac{P}{4 \pi} \frac{x}{R^{3}} .
\end{gathered}
$$

Углы жестких поворотов элементарного куба от объемного деформирования определяются по (1.10): 


$$
\begin{gathered}
\omega_{x}^{0}=\frac{\partial V^{0}}{\partial z}-\frac{\partial W^{0}}{\partial y}=0-K^{0} \frac{P}{4 \pi} \frac{\partial}{\partial y}\left[\frac{1}{\left(x^{2}+y^{2}+z^{2}\right)^{1 / 2}}\right] \\
\omega_{x}^{0}=K^{0} \frac{P}{4 \pi} \frac{y}{R^{3}} \\
\omega_{y}^{0}=\frac{\partial W^{0}}{\partial x}-\frac{\partial U^{0}}{\partial z}=K^{0} \frac{P}{4 \pi} \frac{\partial}{\partial x}\left[\frac{1}{\left(x^{2}+y^{2}+z^{2}\right)^{1 / 2}}\right]-0 \\
\omega_{y}^{0}=K^{0} \frac{P}{4 \pi} \frac{x}{R^{3}} \\
\omega_{z}^{0}=\frac{\partial U_{0}}{\partial y}-\frac{\partial V^{0}}{\partial x}=0+0 .
\end{gathered}
$$

Таким образом, мы получили полное решение задачи о напряженно-деформированном состоянии упругого пространства от сосредоточенной внутри его силы $P$. Как видим, оно отличается от решения для полупространства с сосредоточенной силой постоянным коэффициентом во всех компонентах, равным 0,5, поэтому может быть получено формальным сложением двух полупространств с продлением координатной оси $z$ в отрицательную сторону и умножением всех компонентов решения (4.1)-(4.21) на коэффициент 0,5.

Полученное таким способом решение можно интегрировать по линии и полосе постоянной ширины, конечной и бесконечной длины. При интегрировании по линии и полосе бесконечной длины мы получим плоское деформирование упругого пространства. Эти решения можно получить из приведенных выше решений для полупространства упомянутым способом. В этом смысле решения для полупространства и пространства являются решениями-близнецами. 


\section{3. Плоская деформация бесконечно длинных упругих массивов конечной толщины с ограниченной и неограниченной шириной}

\subsection{1. У Уругий массив ограниченной ширины на жестком основании, допускающем только горизонтальные смещения}

Исходя из условия задачи, граничные условия для функции давления будут следующие (рис. 4.13):

$$
\begin{gathered}
z=0, \quad \frac{\partial \sigma}{\partial z}=0\left(W^{\mathrm{c}}=0\right) \\
z=h, \quad x \neq 0, \quad \sigma=0 ; \quad z=h, \quad x=0, \quad \sigma=\infty,
\end{gathered}
$$

функция должна быть четной относительно координаты $x$, т. е.

$$
\sigma(x, z)=\sigma(-x, z)
$$

Это смешанная задача Дирихле-Неймана, решение которой если имеется, то единственное. Таким решением, удовлетворяющим поставленным условиям, является функция [27]

$$
\sigma=\frac{P}{2 l} \sum_{n=1}^{n=\infty} \cos \frac{\pi(2 n-1) x}{2 l} \frac{\operatorname{ch} \frac{\pi(2 n-1)}{2 l} z}{\operatorname{ch} \frac{\pi(2 n-1)}{2 l} h} .
$$

Для проверки условия, является ли эта функция потенциальной, запишем ее вторые частные производные:

$$
\frac{\partial^{2} \sigma}{\partial z^{2}}=\frac{P}{2 l} \sum_{n=1}^{n=\infty}\left[\frac{\pi(2 n-1) x}{2 l}\right]^{2} \cos \frac{\pi(2 n-1) x}{2 l} \frac{\operatorname{ch} \frac{\pi(2 n-1) z}{2 l}}{\operatorname{ch} \frac{\pi(2 n-1) h}{2 l}}
$$




$$
\frac{\partial^{2} \sigma}{\partial x^{2}}=-\frac{P}{2 l} \sum_{n=1}^{n=\infty}\left[\frac{\pi(2 n-1)}{2 l}\right]^{2} \cos \frac{\pi(2 n-1) x}{2 l} \frac{\operatorname{ch} \frac{\pi(2 n-1) z}{2 l}}{\operatorname{ch} \frac{\pi(2 n-1) h}{2 l}} .
$$

Очевидно, что сумма вторых частных производных равна нулю, поэтому данная функция является гармонической, следовательно, потенциальной.

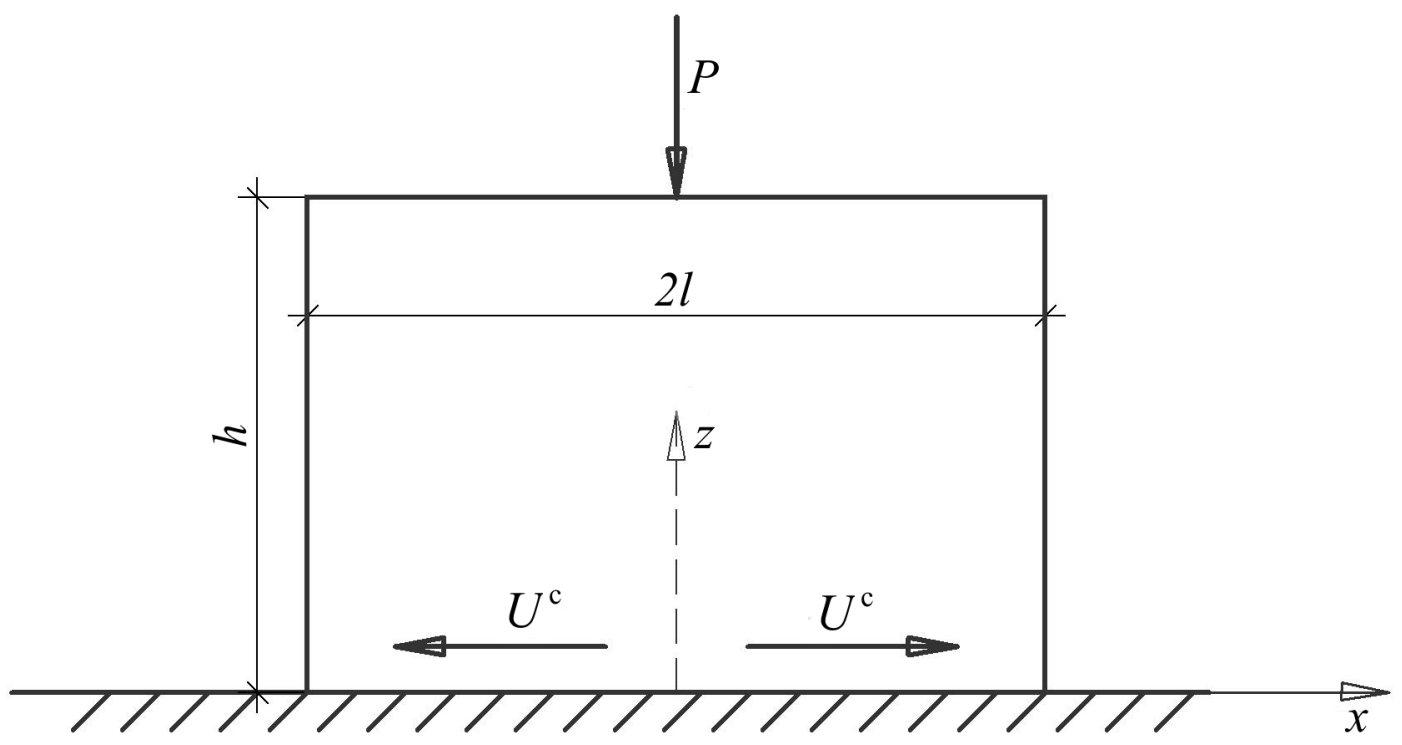

Рис. 4.13. Схема к расчету напряженно-деформированного состояния бесконечно длинного упругого массива прямоугольного сечения на жестком основании, допускающем только горизонтальные смещения чистого сдвига, от линейно распределенной нагрузки

По известной функции давления $\sigma$ (4.92) определяются смещения от чистого сдвига из условий (3.28)-(3.30):

$$
U^{\mathrm{c}}=-K^{\mathrm{c}} \frac{\partial \sigma}{\partial x}=K^{\mathrm{c}} \frac{P}{2 l} \sum_{n=1}^{n=\infty} \frac{\pi(2 n-1)}{2 l} \sin \frac{\pi(2 n-1) x}{2 l} \frac{\operatorname{ch} \frac{\pi(2 n-1) z}{2 l}}{\operatorname{ch} \frac{\pi(2 n-1) h}{2 l}}
$$




$$
W^{\mathrm{c}}=-K^{\mathrm{c}} \frac{\partial \sigma}{\partial z}=-K^{\mathrm{c}} \frac{P}{2 l} \sum_{n=1}^{n=\infty} \frac{\pi(2 n-1)}{2 l} \cos \frac{\pi(2 n-1) x}{2 l} \frac{\operatorname{sh} \frac{\pi(2 n-1) z}{2 l}}{\operatorname{ch} \frac{\pi(2 n-1) h}{2 l}},
$$

а также от объемного сжатия упругого тела из условий (3.18)-(3.20):

$$
W^{0}=K^{0} \int_{z}^{0} \sigma d z=-K^{0} \frac{P}{2 l} \sum_{n=1}^{n=\infty} \frac{2 l}{\pi(2 n-1)} \cos \frac{\pi(2 n-1) x}{2 l} \frac{\operatorname{sh} \frac{\pi(2 n-1) z}{2 l}}{\operatorname{ch} \frac{\pi(2 n-1) h}{2 l}} .
$$

Для случая нагрузки равномерной интенсивности $p\left(\mathrm{H} / \mathrm{m}^{2}\right)$, pacпределенной на бесконечной полосе постоянной ширины $2 a$, функция давления определяется путем интегрирования (4.92) в соответствии со схемой, показанной на рис. 4.14 :

$$
\sigma=\frac{p}{2 l} \int_{-a}^{a} \sum_{n=1}^{n=\infty} \cos \frac{\pi(2 n-1)(x-\varepsilon)}{2 l} \frac{\operatorname{ch} \frac{\pi(2 n-1) z}{2 l}}{\operatorname{ch} \frac{\pi(2 n-1) h}{2 l}} d(x-\varepsilon) .
$$

После интегрирования и алгебраических преобразований имеем:

$$
\sigma=\frac{p}{l} \sum_{n=1}^{n=\infty} \frac{\sin \frac{\pi(2 n-1) a}{2 l}}{\frac{\pi(2 n-1)}{2 l}} \cos \frac{\pi(2 n-1) x}{2 l} \frac{\operatorname{ch} \frac{\pi(2 n-1) z}{2 l}}{\operatorname{ch} \frac{\pi(2 n-1) h}{2 l}} .
$$

По известному значению функции давления (4.93) определяются перемещения в упругом массиве, вызванные чистым формоизменением [29]:

$$
U^{\mathrm{c}}=-K^{\mathrm{c}} \frac{\partial \sigma}{\partial x}=K^{\mathrm{c}} \frac{P}{l} \sum_{n=1}^{n=\infty} \sin \frac{\pi(2 n-1) a}{2 l} \sin \frac{\pi(2 n-1) x}{2 l} \frac{\operatorname{ch} \frac{\pi(2 n-1) z}{2 l}}{\operatorname{ch} \frac{\pi(2 n-1) h}{2 l}}
$$




$$
W^{\mathrm{c}}=-K^{\mathrm{c}} \frac{\partial \sigma}{\partial z}=-K^{\mathrm{c}} \frac{P}{l} \sum_{n=1}^{n=\infty} \sin \frac{\pi(2 n-1) a}{2 l} \cos \frac{\pi(2 n-1) x}{2 l} \frac{\operatorname{sh} \frac{\pi(2 n-1) z}{2 l}}{\operatorname{ch} \frac{\pi(2 n-1) h}{2 l}},
$$

а также вертикальные перемещения от изменения объема среды:

$$
\begin{gathered}
W^{0}=K^{0} \int_{z}^{0} \sigma d z=K^{0} \int_{z}^{0} \frac{P}{l} \sum_{n=1}^{n=\infty} \frac{\sin \frac{\pi(2 n-1) a}{2 l}}{\frac{\pi(2 n-1)}{2 l}} \cos \frac{\pi(2 n-1) x}{2 l} \frac{\operatorname{ch} \frac{\pi(2 n-1) z}{2 l}}{\operatorname{ch} \frac{\pi(2 n-1) h}{2 l}} d z, \\
W^{0}=\frac{-P}{l} \sum_{n=1}^{n=\infty} \frac{\sin \frac{\pi(2 n-1) a}{2 l}}{\left[\frac{\pi(2 n-1)}{2 l}\right]^{2}} \cos \frac{\pi(2 n-1) x}{2 l} \frac{\operatorname{sh} \frac{\pi(2 n-1) z}{2 l}}{\operatorname{ch} \frac{\pi(2 n-1) h}{2 l}} .
\end{gathered}
$$

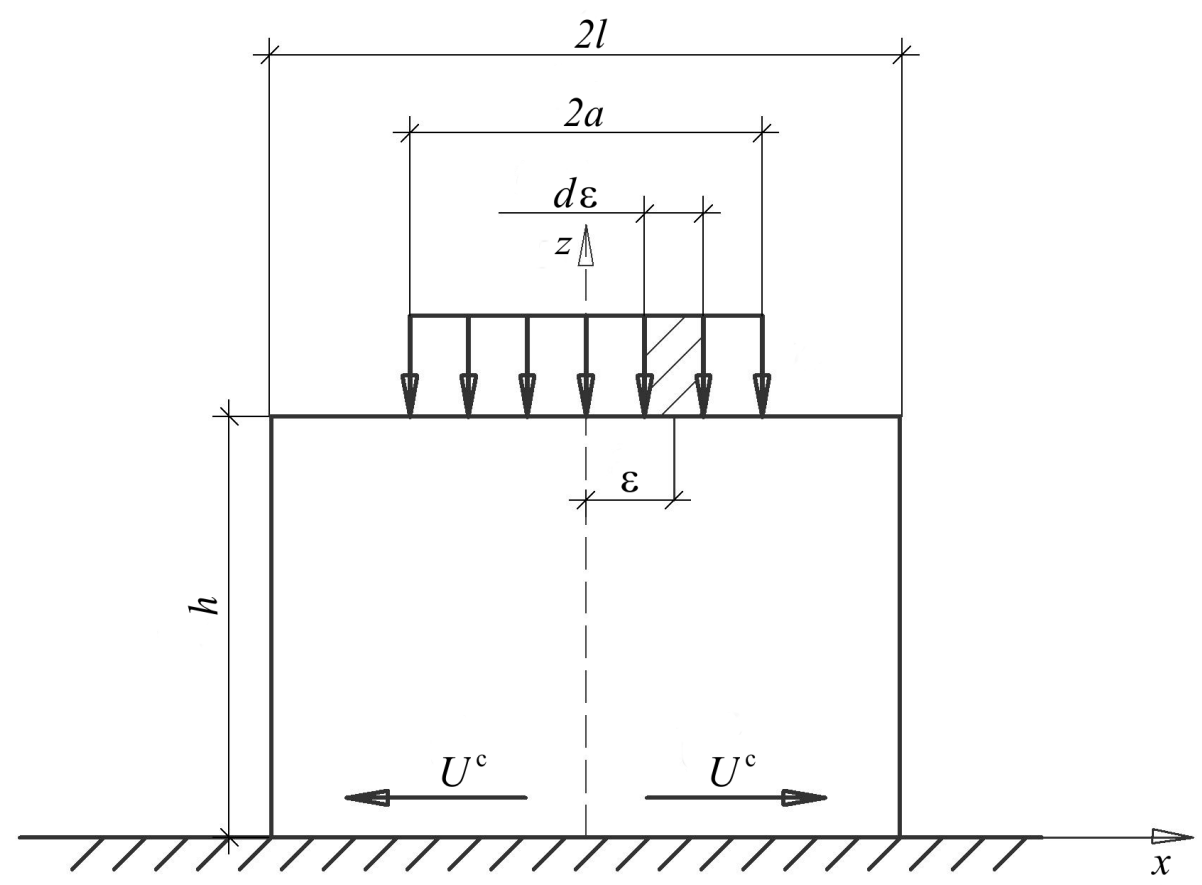

Рис. 4.14. Схема к расчету напряженно-деформированного состояния бесконечно длинного упругого массива прямоугольного сечения на жестком основании, допускающем только горизонтальные смещения чистого сдвига, от полосовой равномерной нагрузки 


\subsection{2. У Уругий массив ограниченной ширины на жестком основании, допускающем только вертикальные смещения}

Рассматриваемый массив и его загружение аналогичны принятым в задаче п. 4.3.1, однако основание допускает только вертикальные смещения от чистой деформации (рис. 4.15). Функция давления должна удовлетворять условиям

$$
\begin{gathered}
z=0, \quad \frac{\partial \sigma}{\partial x}=0 \quad\left(U^{\mathrm{c}}=0\right) ; \quad z=h, \quad x=0, \quad \sigma=\infty ; \quad z=h, \quad x \neq 0, \quad \sigma=0 \\
\sigma(x, z)=\sigma(-x, z) .
\end{gathered}
$$

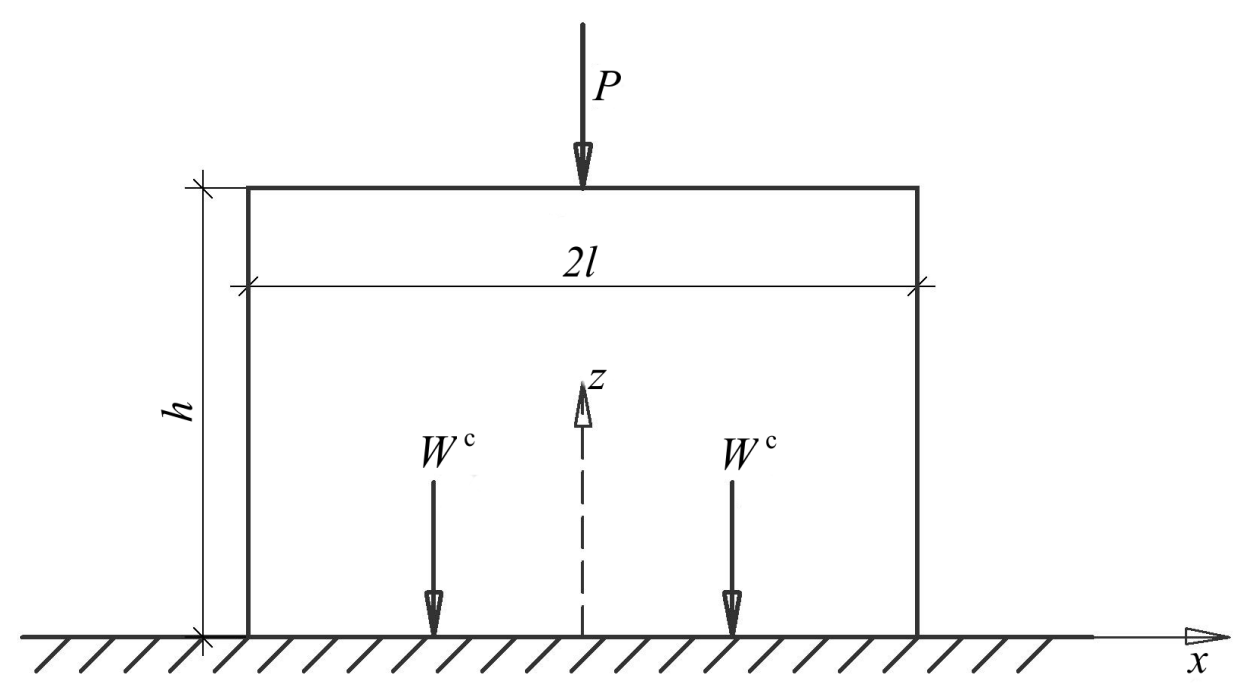

Рис. 4.15. Схема к расчету напряженно-деформированного состояния бесконечно длинного упругого массива прямоугольного сечения на жестком основании, допускающем только вертикальные смещения чистого сдвига, от линейно распределенной нагрузки

Функция давления, удовлетворяющая поставленным граничным условиям, получена в виде [27]

$$
\sigma=\frac{P}{2 l} \sum_{n=1}^{n=\infty} \cos \frac{\pi(2 n-1) x}{2 l} \frac{\operatorname{sh} \frac{\pi(2 n-1) z}{2 l}}{\operatorname{sh} \frac{\pi(2 n-1) h}{2 l}} .
$$


Проверим, является ли данная функция гармонической. Для этого запишем ее вторые частные производные:

$$
\begin{gathered}
\frac{\partial^{2} \sigma}{\partial x^{2}}=-\frac{P}{2 l} \sum_{n=1}^{n=\infty}\left[\frac{\pi(2 n-1)}{2 l}\right]^{2} \cos \frac{\pi(2 n-1) x}{2 l} \frac{\operatorname{sh} \frac{\pi(2 n-1)}{2 l} z}{\operatorname{sh} \frac{\pi(2 n-1)}{2 l} h} \\
\frac{\partial^{2} \sigma}{\partial z^{2}}=\frac{P}{2 l} \sum_{n=1}^{n=\infty}\left[\frac{\pi(2 n-1)}{2 l}\right]^{2} \cos \frac{\pi(2 n-1) x}{2 l} \frac{\operatorname{sh} \frac{\pi(2 n-1)}{2 l} z}{\operatorname{sh} \frac{\pi(2 n-1)}{2 l} h}
\end{gathered}
$$

Очевидно, что сумма этих производных равна нулю, поэтому функция (4.94) является гармонической и, следовательно, потенциальной.

Компоненты перемещений точек среды, вызванные чистым формоизменением, в соответствии с условиями (3.28)-(3.30) будут:

$$
\begin{gathered}
U^{\mathrm{c}}=-K^{\mathrm{c}} \frac{\partial \sigma}{\partial x}=K^{\mathrm{c}} \frac{P}{2 l} \sum_{n=1}^{n=\infty} \frac{\pi(2 n-1)}{2 l} \sin \frac{\pi(2 n-1) x}{2 l} \frac{\operatorname{sh} \frac{\pi(2 n-1) z}{2 l}}{\operatorname{sh} \frac{\pi(2 n-1) h}{2 l}} \\
W^{\mathrm{c}}=-K^{\mathrm{c}} \frac{\partial \sigma}{\partial x}=-\frac{P}{2 l} \sum_{n=1}^{n=\infty} \frac{\pi(2 n-1)}{2 l} \cos \frac{\pi(2 n-1) x}{2 l} \frac{\operatorname{ch} \frac{\pi(2 n-1) z}{2 l}}{\operatorname{sh} \frac{\pi(2 n-1) h}{2 l}} .
\end{gathered}
$$

Перемещения, вызванные изменением плотности среды, определяются интегрированием (4.94) в соответствии с (3.17)-(3.19):

$$
W^{0}=\frac{K^{0} P}{2 l} \int \sum_{n=1}^{n=\infty} \cos \frac{\pi(2 n-1) x}{2 l} \frac{\operatorname{sh} \frac{\pi(2 n-1) z}{2 l}}{\operatorname{sh} \frac{\pi(2 n-1) h}{2 l}} d z,
$$




$$
W^{0}=\frac{K^{0} P}{2 l} \sum_{n=1}^{n=\infty} \cos \frac{\pi(2 n-1) x}{2 l} \frac{\operatorname{sh} \frac{\pi(2 n-1) z}{2 l}}{\operatorname{sh} \frac{\pi(2 n-1) h}{2 l}}+C .
$$

Произвольная интегрирования $C$ определяется из граничных условий: при $z=0$ перемещения от изменения плотности $W^{0}=0$, тогда окончательно имеем

$$
W^{0}=-K^{0} P \sum_{n=1}^{n=\infty} \frac{1}{\pi(2 n-1)} \cos \frac{\pi(2 n-1) x}{2 l} \frac{\left[\operatorname{ch} \frac{\pi(2 n-1) z}{2 l}-1\right]}{\operatorname{sh} \frac{\pi(2 n-1) h}{2 l}} .
$$

В том случае когда на поверхности массива на полосе шириной $2 a$ будет действовать равномерно распределенная нагрузка $p\left(\mathrm{H} / \mathrm{m}^{2}\right)$, функция давления определяется интегрированием (4.94) в соответствии со схемой, приведенной на рис. 4.16:

$$
\begin{gathered}
\sigma=\frac{p}{2 l} \int_{-a}^{a} \sum_{n=1}^{n=\infty} \cos \frac{\pi(2 n-1)(x-\varepsilon)}{2 l} \frac{\operatorname{sh} \frac{\pi(2 n-1) z}{2 l}}{\operatorname{sh} \frac{\pi(2 n-1) h}{2 l}} d(x-\varepsilon), \\
\sigma=-\left.\frac{p}{2 l} \sum_{n=1}^{n=\infty} \frac{2 l}{\pi(2 n-1)} \sin \frac{\pi(2 n-1)(x-\varepsilon)}{2 l} \frac{\operatorname{sh} \frac{\pi(2 n-1) z}{2 l}}{\operatorname{sh} \frac{\pi(2 n-1) h}{2 l}}\right|_{-a} ^{a} .
\end{gathered}
$$

После подстановки пределов и алгебраических преобразований функция давления принимает вид

$$
\sigma=\frac{p}{l} \sum_{n=1}^{n=8} \frac{\sin \frac{\pi(2 n-1) a}{2 l}}{\frac{\pi(2 n-1)}{2 l}} \cos \frac{\pi(2 n-1) x}{2 l} \frac{\operatorname{sh} \frac{\pi(2 n-1) z}{2 l}}{\operatorname{sh} \frac{\pi(2 n-1) h}{2 l}} .
$$


По известной функции давления (4.96) определяются смещения точек массива, вызванные чистым формоизменением:

$$
\begin{gathered}
U^{\mathrm{c}}=-K^{\mathrm{c}} \frac{\partial \sigma}{\partial x}=K^{\mathrm{c}} \frac{p}{l} \sum_{n=1}^{n=\infty} \sin \frac{\pi(2 n-1) a}{2 l} \sin \frac{\pi(2 n-1) x}{2 l} \frac{\operatorname{sh} \frac{\pi(2 n-1) z}{2 l}}{\operatorname{sh} \frac{\pi(2 n-1) h}{2 l}} \\
W^{\mathrm{c}}=-K^{\mathrm{c}} \frac{\partial \sigma}{\partial z}=-K^{\mathrm{c}} \frac{p}{l} \sum_{n=1}^{n=\infty} \sin \frac{\pi(2 n-1) a}{2 l} \cos \frac{\pi(2 n-1) x}{2 l} \frac{\operatorname{ch} \frac{\pi(2 n-1) z}{2 l}}{\operatorname{ch} \frac{\pi(2 n-1) h}{2 l}} .
\end{gathered}
$$

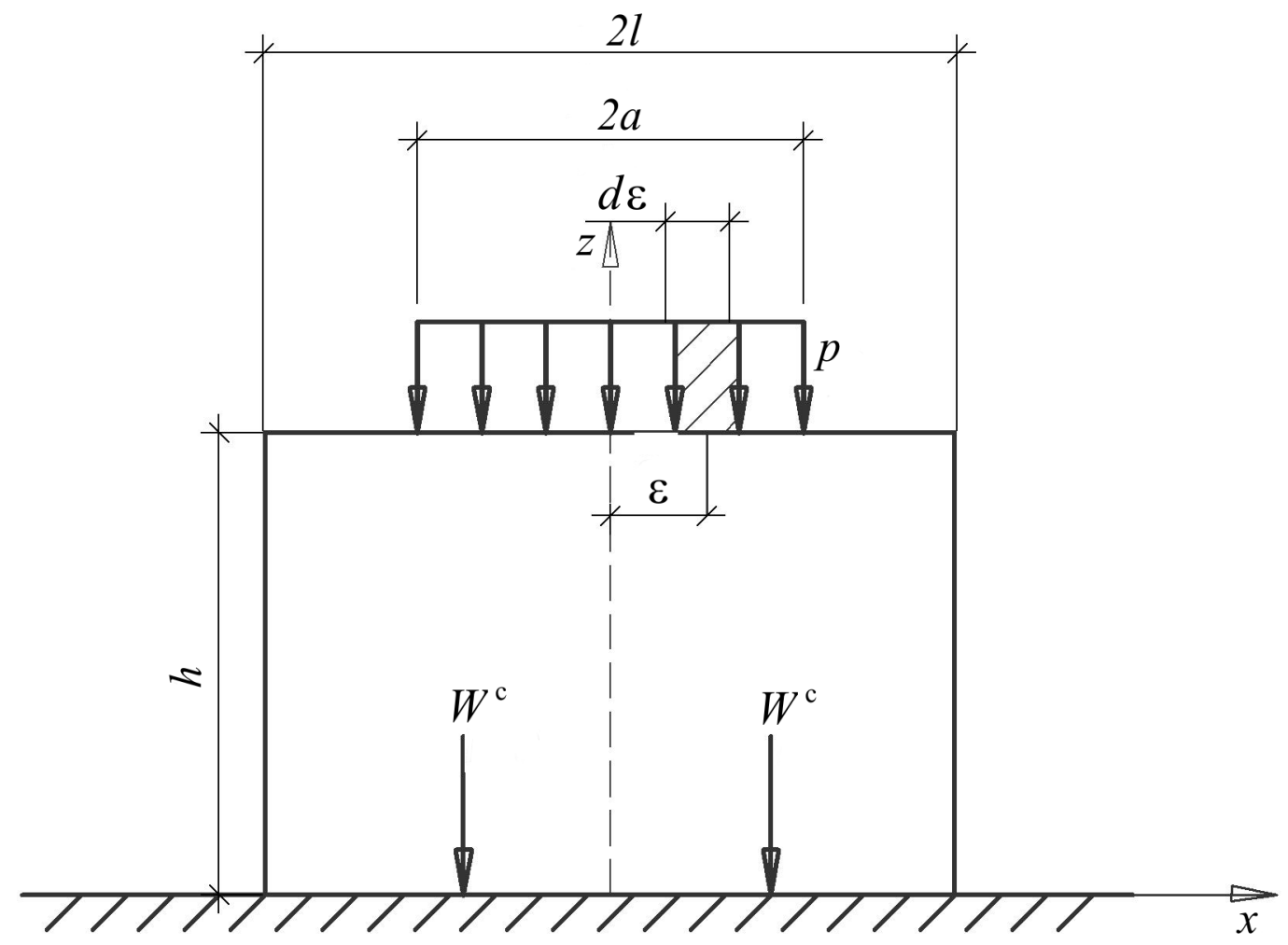

Рис. 4.16. Схема к расчету напряженно-деформированного состояния бесконечно длинного упругого массива прямоугольного сечения на жестком основании, допускающем только вертикальные смещения чистого сдвига, от полосовой равномерной нагрузки 
Перемещения точек упругой среды от изменения ее плотности получим интегрированием (4.96) с учетом (3.17)-(3.19) и граничных условий, подобно тому, как это выполнялось в (4.95):

$$
W^{0}=-K^{0} \frac{p}{2 l} \sum_{n=1}^{n=\infty} \frac{\sin \frac{\pi(2 n-1) a}{2 l}}{\left[\frac{\pi(2 n-1)}{2 l}\right]^{2}} \cos \frac{\pi(2 n-1) x}{2 l} \frac{\left[\operatorname{ch} \frac{\pi(2 n-1)}{2 l}-1\right]}{\operatorname{sh} \frac{\pi(2 n-1) h}{2 l}} .
$$

\subsection{3. Упругий массив ограниченной ширины на жестком основании, допускающем частично горизонтальные и вертикальные смещения}

В задачах п. 4.3.1 и 4.3.2 рассматривалось напряженно-деформированное состояние упругих массивов прямоугольного поперечного сечения бесконечной длины для двух предельных случаев по граничным условиям на контакте их с основанием: контакт допускает только горизонтальные смещения от чистого сдвига (п. 4.3.1) либо только вертикальные (п. 4.3.2). Если же контакт с основанием допускает частично горизонтальные и вертикальные смещения, то задача может быть решена путем комбинирования двух этих решений. С этой целью внешняя нагрузка делится на две части пропорционально оценочной величине упомянутых смещений:

$$
p=p^{\Gamma}+p^{\mathrm{B}}=m^{\Gamma} p+m^{\mathrm{B}} p=p\left(m^{\Gamma}+m^{\mathrm{B}}\right),
$$

где $m^{\text {г }}$ и $m^{\text {в }}$ - коэффициенты, пропорциональные частям внешней нагрузки, формирующим соответственно горизонтальные и вертикальные смещения точек упругой среды от чистого сдвига. Очевидно, что их сумма равна единице.

Таким образом, общее решение будет состоять из суммы двух решений: решения (п. 4.3.1) с коэффициентом пропорциональности $m^{\text {г }}$ и решения (п. 4.3.2) с коэффициентом пропорциональности $m^{\text {в } п р и ~}$ соответствующей внешней нагрузке. Например, для нагрузки $P$, равномерно распределенной вдоль бесконечной линии, будем иметь следующее решение: 
функция давления

$$
\sigma=\frac{P}{2 l} \sum_{n=1}^{n=8} \cos \frac{\pi(1 n-1) x}{2 l}\left[m^{\mathrm{\Gamma}} \frac{\operatorname{ch} \frac{\pi(2 n-1) z}{2 l}}{\operatorname{ch} \frac{\pi(2 n-1) h}{2 l}}+m^{\mathrm{B}} \frac{\operatorname{sh} \frac{\pi(2 n-1) z}{2 l}}{\operatorname{sh} \frac{\pi(2 n-1) h}{2 l}}\right],
$$

перемещения, вызванные чистым формоизменением среды,

$$
\begin{aligned}
\left.U^{\mathrm{c}}=K^{\mathrm{c}} \frac{P}{2 l} \sum_{n=1}^{n=\infty} \frac{\pi(2 n-1)}{2 l} \sin \frac{\pi(2 n}{2 l}-1\right) x & \times \\
& \times\left[\begin{array}{cc}
\left.m^{\mathrm{\Gamma}} \frac{\operatorname{ch} \frac{\pi(2 n-1) z}{2 l}}{\operatorname{ch} \frac{\pi(2 n-1) h}{2 l}}+m^{\mathrm{B}} \frac{\operatorname{sh} \frac{\pi(2 n-1) z}{2 l}}{\operatorname{sh} \frac{\pi(2 n-1) h}{2 l}}\right] ;
\end{array}\right.
\end{aligned}
$$$$
W^{\mathrm{c}}=K^{\mathrm{c}} \frac{P}{2 l} \sum_{n=1}^{n=\infty} \frac{\pi(2 n-1)}{2 l} \cos \frac{\pi(2 n-1) x}{2 l} \times
$$$$
\times\left[m^{\Gamma} \frac{\operatorname{sh} \frac{\pi(2 n-1) z}{2 l}}{\operatorname{ch} \frac{\pi(2 n-1) h}{2 l}}+m^{\mathrm{B}} \frac{\operatorname{ch} \frac{\pi(2 n-1) z}{2 l}}{\operatorname{sh} \frac{\pi(2 n-1) h}{2 l}}\right]
$$

перемещения, вызванные изменением объема среды,

$$
\begin{aligned}
& W^{0}=-K^{0} \frac{P}{2 l} \sum_{n=1}^{n=\infty} \frac{\pi(2 n-1)}{2 l} \cos \frac{\pi(2 n-1) x}{2 l} \times \\
& \times\left[m^{\mathrm{\Gamma}} \frac{\operatorname{sh} \frac{\pi(2 n-1) z}{2 l}}{\operatorname{ch} \frac{\pi(2 n-1) h}{2 l}}+m^{\mathrm{B}} \frac{\operatorname{ch} \frac{\pi(2 n-1) z}{2 l}}{\operatorname{sh} \frac{\pi(2 n-1) h}{2 l}}\right] .
\end{aligned}
$$


Для случая нагрузки $p\left(\mathrm{H} / \mathrm{m}^{2}\right)$, равномерно распределенной вдоль бесконечной полосы шириной $2 a$, получим следующее решение:

функция давления

$$
\sigma=\frac{p}{l} \sum_{n=1}^{n=\infty} \frac{\sin \frac{\pi(2 n-1) a}{2 l}}{\frac{\pi(2 n-1)}{2 l}} \cos \frac{\pi(2 n-1) x}{2 l} \times
$$

$$
\times\left[m^{\mathrm{r}} \frac{\operatorname{ch} \frac{\pi(2 n-1) z}{2 l}}{\operatorname{ch} \frac{\pi(2 n-1) h}{2 l}}+m^{\mathrm{B}} \frac{\operatorname{sh} \frac{\pi(2 n-1) z}{2 l}}{\operatorname{sh} \frac{\pi(2 n-1) h}{2 l}}\right] ;
$$

смещения точек упругого массива от чистого формоизменения

$$
\begin{aligned}
& U^{\mathrm{c}}=K^{\mathrm{c}} \frac{p}{l} \sum_{n=1}^{n=\infty} \sin \frac{\pi(2 n-1) a}{2 l} \sin \frac{\pi(2 n-1) x}{2 l} \times \\
& \times\left[m^{\mathrm{r}} \frac{\operatorname{ch} \frac{\pi(2 n-1) z}{2 l}}{\operatorname{ch} \frac{\pi(2 n-1) h}{2 l}}+m^{\mathrm{B}} \frac{\operatorname{sh} \frac{\pi(2 n-1) z}{2 l}}{\operatorname{sh} \frac{\pi(2 n-1) h}{2 l}}\right],
\end{aligned}
$$

$W^{\mathrm{c}}=-K^{\mathrm{c}} \frac{p}{l} \sin \sum_{n=1}^{n=\infty} \frac{\pi(2 n-1) a}{2 l} \cos \frac{\pi(2 n-1) x}{2 l} \times$

$$
\times\left[m^{\mathrm{r}} \frac{\operatorname{sh} \frac{\pi(2 n-1) z}{2 l}}{\operatorname{ch} \frac{\pi(2 n-1) h}{2 l}}+m^{\mathrm{B}} \frac{\operatorname{ch} \frac{\pi(2 n-1) z}{2 l}}{\operatorname{sh} \frac{\pi(2 n-1) h}{2 l}}\right] ;
$$


смещение точек упругого массива от изменения его плотности:

$$
\begin{aligned}
W^{0}=-K^{0} \frac{p}{l} \sum_{n=1}^{n=\infty} \frac{\sin \frac{\pi(2 n-1) a}{2 l}}{\left[\frac{\pi(2 n-1)}{2 l}\right]^{2}} \cos \frac{\pi(2 n-1) x}{2 l} \times \\
\times\left\{m^{\mathrm{r}} \frac{\operatorname{sh} \frac{\pi(2 n-1) z}{2 l}}{\operatorname{ch} \frac{\pi(2 n-1) h}{2 l}}+m^{\mathrm{B}} \frac{\left[\operatorname{ch} \frac{\pi(2 n-1) z}{2 l}-1\right]}{\operatorname{sh} \frac{\pi(2 n-1) h}{2 l}}\right\} .
\end{aligned}
$$

\subsection{4. Бесконечно простирающийся упругий массив на жестком основании, допускающем только горизонтальные смещения}

По нормали к поверхности массива приложена нагрузка $P(\mathrm{H} / \mathrm{M})$, равномерно распределенная вдоль бесконечной линии (рис. 4.17). Условия контакта массива с жестким основанием допускают только горизонтальные смещения. Граничными условиями для функции давления в этом случае будут:

$$
\begin{gathered}
z=0, \quad \frac{\partial \sigma}{\partial z}=0 \quad\left(W^{\mathrm{c}}=0\right) \\
z=h, \quad x \neq 0, \quad \sigma=0 ; \quad z=h, \quad x=0, \quad \sigma=\infty .
\end{gathered}
$$

Для определения такой функции давления воспользуемся следующим приемом. В решении (4.92) будем увеличивать ширину массива, устремив ее к бесконечности $(l \rightarrow \infty)$ В пределе мы должны получить искомую функцию. Для этого представим функцию (4.92) в следующем виде:

$$
\sigma_{\text {огр }} \frac{P}{2 l} \sum_{n=1}^{n=\infty} \frac{\operatorname{ch}\left(z t_{n}\right)}{\operatorname{ch}\left(h t_{n}\right)} \cos \left(x t_{n}\right) \Delta t_{n},
$$


где

$$
t_{n}=\frac{\pi(2 n-1)}{2 l}
$$

При неограниченном увеличении ширины массива $(l \rightarrow \infty)$ суммирование заменяем интегрированием:

$$
\sigma=\lim _{x \rightarrow \infty} \sigma_{\text {огр }}=\frac{P}{\pi} \int_{0}^{\infty} \frac{\operatorname{ch}(z t)}{\operatorname{ch}(h t)} \cos (x t) d t .
$$

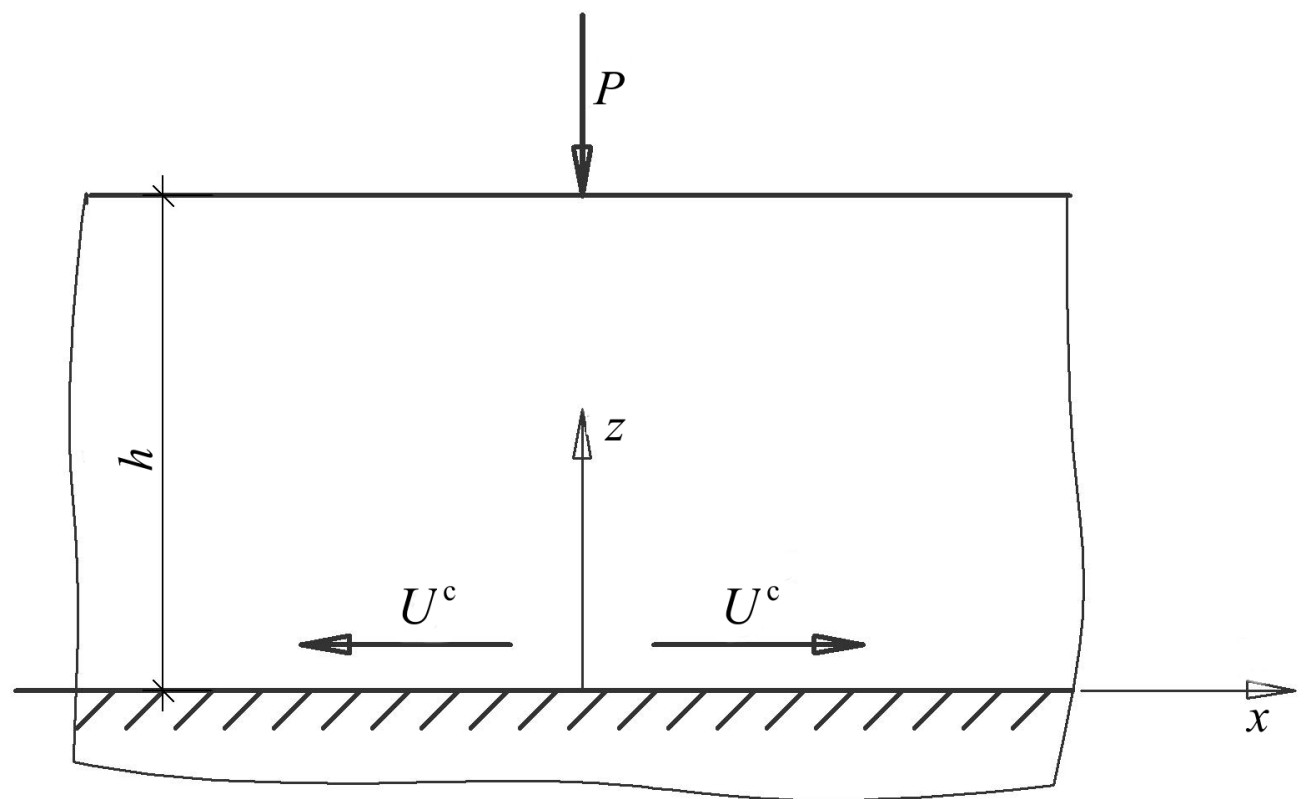

Рис. 4.17. Схема к расчету напряженно-деформированного состояния бесконечно простирающегося массива ограниченной толщины на жестком основании, допускающем только горизонтальные перемещения чистого сдвига, от линейно-распределенной нагрузки

Согласно [30] несобственный интеграл такого вида равен

$$
\int_{0}^{\infty} \frac{\operatorname{ch} \beta x}{\operatorname{ch} \gamma x} \cos \alpha_{x} d x=\frac{\pi}{\gamma} \frac{\cos \frac{\pi \beta}{2 \gamma} \operatorname{ch} \frac{\pi \alpha}{2 \gamma}}{\operatorname{ch} \frac{\pi \alpha}{\gamma}+\cos \frac{\pi \beta}{\gamma}} .
$$


Выполнив соответствующую подстановку, будем иметь функцию давления

$$
\sigma=\frac{P}{h} \frac{\operatorname{ch} \frac{\pi x}{2 h} \cos \frac{\pi z}{2 h}}{\operatorname{ch} \frac{\pi x}{h}+\cos \frac{\pi z}{h}} .
$$

Путем элементарных алгебраических преобразований приводим ее к более удобному виду [29]

$$
\sigma=\frac{P}{2 h} \frac{\operatorname{ch} \frac{\pi x}{2 h} \cos \frac{\pi z}{2 h}}{\operatorname{sh}^{2} \frac{\pi x}{2 h}+\cos ^{2} \frac{\pi z}{h}}
$$

или

$$
\sigma=\frac{P}{2 h} \frac{\operatorname{ch} \frac{\pi x}{2 h} \cos \frac{\pi z}{2 h}}{\operatorname{ch}^{2} \frac{\pi x}{2 h}-\sin ^{2} \frac{\pi z}{h}} .
$$

Можно убедиться, что данная функция давления удовлетворяет поставленным граничным условиям и является гармонической, т. е. обладает потенциалом. Здесь следует отметить, что функция (4.92) была получена в [27] при исследовании мгновенных напоров воды в грунтовой массе после приложения нагрузки.

Если предпосылки к получению функций давления (4.92) и (4.98)(4.100) корректны, а сами функции определены верно, то путем предельного перехода $h \rightarrow \infty$ от функции, например (4.98), мы должны получить функцию давления для случая плоского деформирования полупространства линейно распределенной нагрузкой.

Предварительно в целях упрощения выкладок мысленно перенесем начало координат (см. рис. 4.13) на поверхность массива, в точку приложения силы и попытаемся перейти к упомянутому пределу: 


$$
\sigma=\lim _{h \rightarrow \infty} \sigma_{\text {огр }}=\lim _{h \rightarrow \infty} \frac{P}{\pi} \frac{\operatorname{ch} \frac{\pi x}{2 h} \cos \frac{\pi(h-z)}{2 h}}{\operatorname{ch} \frac{\pi x}{h}+\cos \frac{\pi(h-z)}{h}}=\frac{0}{0} .
$$

Для раскрытия неопределенности воспользуемся правилом Лопиталя

$$
\lim _{h \rightarrow \infty} \frac{P}{h} \frac{\operatorname{sh} \frac{\pi x}{2 h}\left(-\frac{\pi x}{2 h^{2}}\right) \cos \frac{\pi(h-z)}{2 h}-\operatorname{ch} \frac{\pi x}{2 h} \sin \frac{\pi(h-z)}{2 h}\left(\frac{\pi z}{2 h^{2}}\right)}{\operatorname{sh} \frac{\pi x}{h}\left(-\frac{\pi x}{2 h^{2}}\right)-\sin \frac{\pi(h-z)}{h}\left(\frac{\pi z}{h^{2}}\right)}=\frac{0}{0} .
$$

Поскольку неопределенность остается нераскрытой, воспользуемся второй раз этим правилом и запишем раздельно производные числителя и знаменателя.

Производная числителя по $h$ :

$$
\begin{array}{r}
P\left[\operatorname{ch} \frac{\pi x}{2 h}\left(-\frac{\pi x}{2 h^{2}}\right) \cos \frac{\pi(h-z)}{2 h}+\operatorname{sh} \frac{\pi x}{2 h}\left(\frac{\pi x}{h^{3}}\right) \cos \frac{\pi(h-z)}{2 h}-\right. \\
-\operatorname{sh} \frac{\pi x}{2 h}\left(-\frac{\pi x}{2 h^{2}}\right)^{2} \sin \frac{\pi(h-z)}{2 h}\left(\frac{\pi z}{2 h^{2}}\right)-\operatorname{sh} \frac{\pi x}{2 h} \sin \frac{\pi(h-z)}{2 h}\left(\frac{\pi z}{2 h^{2}}\right)- \\
\left.-\operatorname{ch} \frac{\pi x}{2 h} \cos \frac{\pi(h-z)}{2 h}\left(\frac{\pi z}{2 h^{2}}\right)^{2}-\operatorname{ch} \frac{\pi x}{2 h} \sin \frac{\pi(h-z)}{2 h}\left(-\frac{\pi x}{h^{3}}\right)\right] .
\end{array}
$$

Производная знаменателя по $h$ :

$$
h\left[\operatorname{ch} \frac{\pi x}{h}\left(\frac{\pi x}{h^{2}}\right)^{2}+\operatorname{ch} \frac{\pi x}{h}\left(\frac{2 \pi x}{h^{3}}\right)-\cos \frac{\pi(h-z)}{h}\left(\frac{\pi z}{h^{2}}\right)^{2}-\sin \frac{\pi(h-z)}{h}\left(\frac{\pi z}{h^{3}}\right)\right] .
$$


После упрощений числителя и знаменателя получаем

$$
\lim _{h \rightarrow \infty} \frac{P}{h} \frac{\frac{\pi}{h^{3}} z}{\frac{\pi^{2}}{h^{4}}\left(x^{2}+z^{2}\right)}=\frac{P}{\pi} \frac{z}{\left(x^{2}+z^{2}\right)} .
$$

Таким образом, мы получили функцию давления при нагружении полупространства нормальной к его поверхности нагрузкой, равномерно распределенной вдоль бесконечной линии (4.60) и подтвердили взаимосвязь функций давления (4.60), (4.92), (4.94) и (4.99).

Для определения компонентов напряжений и смещений точек упругого массива в данной задаче запишем частные производные функции давления (4.99):

$$
\begin{aligned}
& \frac{\partial}{\partial x}\left(\frac{P}{2 h} \frac{\operatorname{ch} \frac{\pi x}{2 h} \cos \frac{\pi z}{2 h}}{\operatorname{sh}^{2} \frac{\pi x}{2 h}+\cos ^{2} \frac{\pi z}{2 h}}\right)=-\frac{P \pi}{4 h^{2}} \frac{\operatorname{sh} \frac{\pi x}{2 h} \cos \frac{\pi z}{2 h}\left(\operatorname{ch}^{2} \frac{\pi x}{2 h}-\sin ^{2} \frac{\pi z}{2 h}\right)}{\left(\operatorname{sh}^{2} \frac{\pi x}{2 h}+\cos ^{2} \frac{\pi z}{2 h}\right)^{2}} \\
& \frac{\partial}{\partial z}\left(\frac{P}{2 h} \frac{\operatorname{ch} \frac{\pi x}{2 h} \cos \frac{\pi z}{2 h}}{\operatorname{sh}^{2} \frac{\pi x}{2 h}+\cos ^{2} \frac{\pi z}{2 h}}\right)=-\frac{P \pi}{4 h^{2}} \frac{\operatorname{ch} \frac{\pi x}{2 h} \sin \frac{\pi z}{2 h}\left(\operatorname{sh}^{2} \frac{\pi x}{2 h}-\cos ^{2} \frac{\pi z}{2 h}\right)}{\left(\operatorname{sh}^{2} \frac{\pi x}{2 h}+\cos ^{2} \frac{\pi z}{2 h}\right)^{2}}
\end{aligned}
$$

Компоненты напряжений могут быть определены по зависимостям (3.39):

$$
\begin{gathered}
\sigma_{x}=\sigma+(h-z) \frac{\partial \sigma}{\partial z} \\
\sigma_{x}=\frac{P}{2 h}\left[\frac{\operatorname{ch} \frac{\pi x}{2 h} \cos \frac{\pi z}{2 h}}{\operatorname{sh}^{2} \frac{\pi x}{2 h}+\cos ^{2} \frac{\pi z}{2 h}}-\frac{\pi(h-z)}{2 h} \frac{\operatorname{ch} \frac{\pi x}{2 h} \sin \frac{\pi z}{2 h}\left(\operatorname{sh}^{2} \frac{\pi x}{2 h}-\cos ^{2} \frac{\pi z}{2 h}\right)}{\left(\operatorname{sh}^{2} \frac{\pi x}{2 h}+\cos ^{2} \frac{\pi z}{2 h}\right)^{2}}\right] ;
\end{gathered}
$$




$$
\left.\begin{array}{c}
\sigma_{z}=\sigma-(h-z) \frac{\partial \sigma}{\partial z} \\
\sigma_{z}=\frac{P}{2 h}\left[\frac{\operatorname{ch} \frac{\pi x}{2 h} \cos \frac{\pi z}{2 h}}{\operatorname{sh}^{2} \frac{\pi x}{2 h}+\cos ^{2} \frac{\pi z}{2 h}}+\right. \\
\left.+\frac{\pi(h-z)}{2 h} \frac{\operatorname{ch} \frac{\pi x}{2 h} \sin \frac{\pi z}{2 h}\left(\operatorname{sh}^{2} \frac{\pi x}{2 h}-\cos ^{2} \frac{\pi z}{2 h}\right)}{\left(\operatorname{sh}^{2} \frac{\pi x}{2 h}+\cos ^{2} \frac{\pi z}{2 h}\right)^{2}}\right] \\
\sigma_{y}=\sigma=\frac{P}{2 h}\left(\frac{\left.\operatorname{ch}^{2} \frac{\pi x}{2 h} \cos ^{2} \frac{\pi z}{2 h}\right)}{\left.\operatorname{sh}^{2} \frac{\pi x}{2 h}+\cos ^{2} \frac{\pi z}{2 h}\right)^{2}}\right) \\
=-\frac{P \pi}{4 h^{2}}(h-z) \frac{(h-z) \frac{\partial \sigma}{\partial x}}{\tau_{z x}}
\end{array}\right]
$$

Для проверки условия соблюдения общего равновесия в данной задаче выполним следующую операцию: просуммируем вертикальное давление, которое передается в любой точке от массива на основание, в результате чего оно должно быть равным приложенной силе $P$.

Приняв в (4.101) $z=0$, имеем:

$$
\sigma_{z}=\frac{P}{2 h} \frac{\operatorname{ch} \frac{\pi x}{2 h}}{\operatorname{sh}^{2} \frac{\pi x}{2 h}+1} .
$$


Теперь проинтегрируем $\sigma_{z}$ по контакту упругого массива с основанием

$$
2 \frac{P}{2 h} \int_{0}^{\infty} \frac{\operatorname{ch} \frac{\pi x}{2 h} d x}{\operatorname{sh}^{2} \frac{\pi x}{2 h}+1}=\frac{p}{h} \frac{2 h}{\pi} \int_{0}^{\infty} \frac{d\left(\operatorname{sh} \frac{\pi x}{2 h}\right)}{\operatorname{sh}^{2} \frac{\pi x}{2 h}+1}=\left.\frac{2 P}{\pi} \operatorname{arctg}\left(\operatorname{sh} \frac{\pi x}{2}\right)\right|_{0} ^{\infty}=\frac{2 P}{\pi}\left(\frac{\pi}{2}-1\right)=P
$$

то есть условие общего равновесия в задаче выполняется.

Компоненты смещений точек упругого массива от чистого формоизменения получим согласно (3.28)-(3.30):

$$
\begin{aligned}
& U^{\mathrm{c}}=K^{\mathrm{c}} \frac{P \pi}{4 h^{2}} \frac{\operatorname{sh} \frac{\pi x}{2 h} \cos \frac{\pi z}{2 h}\left(\operatorname{ch}^{2} \frac{\pi x}{2 h}-\sin ^{2} \frac{\pi z}{2 h}\right)}{\left(\operatorname{sh}^{2} \frac{\pi x}{2 h}+\cos ^{2} \frac{\pi z}{2 h}\right)^{2}} ; \\
& W^{\mathrm{c}}=K^{\mathrm{c}} \frac{P \pi}{4 h^{2}} \frac{\operatorname{ch} \frac{\pi x}{2 h} \sin \frac{\pi z}{2 h}\left(\operatorname{sh}^{2} \frac{\pi x}{2 h}-\cos ^{2} \frac{\pi z}{2 h}\right)}{\left(\operatorname{sh}^{2} \frac{\pi x}{2 h}+\cos ^{2} \frac{\pi z}{2 h}\right)^{2}} .
\end{aligned}
$$

Смещения точек упругого массива от изменения объема будут:

$$
\begin{gathered}
\int_{0}^{z} \sigma d z=K^{0} \frac{P}{2 h} \int_{0}^{z} \frac{\operatorname{ch} \frac{\pi x}{2 h} \cos \frac{\pi z}{2 h} d z}{\operatorname{ch} \frac{\pi x^{2}}{2 h}-\sin ^{2} \frac{\pi z}{2 h}}=-K^{0} \frac{P}{2 \pi} \ln \left|\frac{\operatorname{ch} \frac{\pi x}{2 h}+\sin \frac{\pi z}{2 h}}{\operatorname{ch} \frac{\pi x}{2 h}-\sin \frac{\pi z}{2 h}}\right|_{0}^{z} \\
W^{0}=-K^{0} \frac{P}{2 \pi} \ln \left|\frac{\operatorname{ch} \frac{\pi x}{2 h}+\sin \frac{\pi z}{2 h}}{\operatorname{ch} \frac{\pi x}{2 h}-\sin \frac{\pi z}{2 h}}\right|
\end{gathered}
$$

Для случая, когда на поверхности упругого массива будет действовать равномерно распределенная нагрузка интенсивностью $p, \mathrm{H} / \mathrm{m}^{2}$, 
по бесконечной полосе шириной $2 a$, функция давления может быть получена путем интегрирования (4.99) соответственно рис. 4.18:

$$
\begin{aligned}
& \sigma=\frac{p}{2 h} \int_{-a}^{a} \frac{\operatorname{ch} \frac{\pi(x-\varepsilon)}{2 h} \cos \frac{\pi z}{2 h} d(x-\varepsilon)}{\operatorname{ch}^{2} \frac{\pi(x-\varepsilon)}{2 h}+\cos ^{2} \frac{\pi z}{2 h}}= \\
& =\frac{p}{2 h} \frac{2 h}{\pi} \cos \frac{\pi z}{2 h} \int_{-a}^{a} \frac{d \operatorname{sh}\left[\frac{\pi(x-\varepsilon)}{2 h}\right]}{\operatorname{sh}^{2} \frac{\pi(x-\varepsilon)}{2 h}+\cos ^{2} \frac{\pi z}{2 h}}=\left.\frac{p}{\pi} \operatorname{arctg} \frac{\operatorname{sh} \frac{\pi(x-\varepsilon)}{2 h}}{\cos \frac{\pi z}{2 h}}\right|_{-a} ^{a}, \\
& \sigma=\frac{p}{2 h}\left[\operatorname{arctg} \frac{\operatorname{ch} \frac{\pi(x+a)}{2 h}}{\cos \frac{\pi z}{2 h}}-\operatorname{arctg} \frac{\operatorname{sh} \frac{\pi(x-a)}{2 h}}{\cos \frac{\pi z}{2 h}}\right] .
\end{aligned}
$$

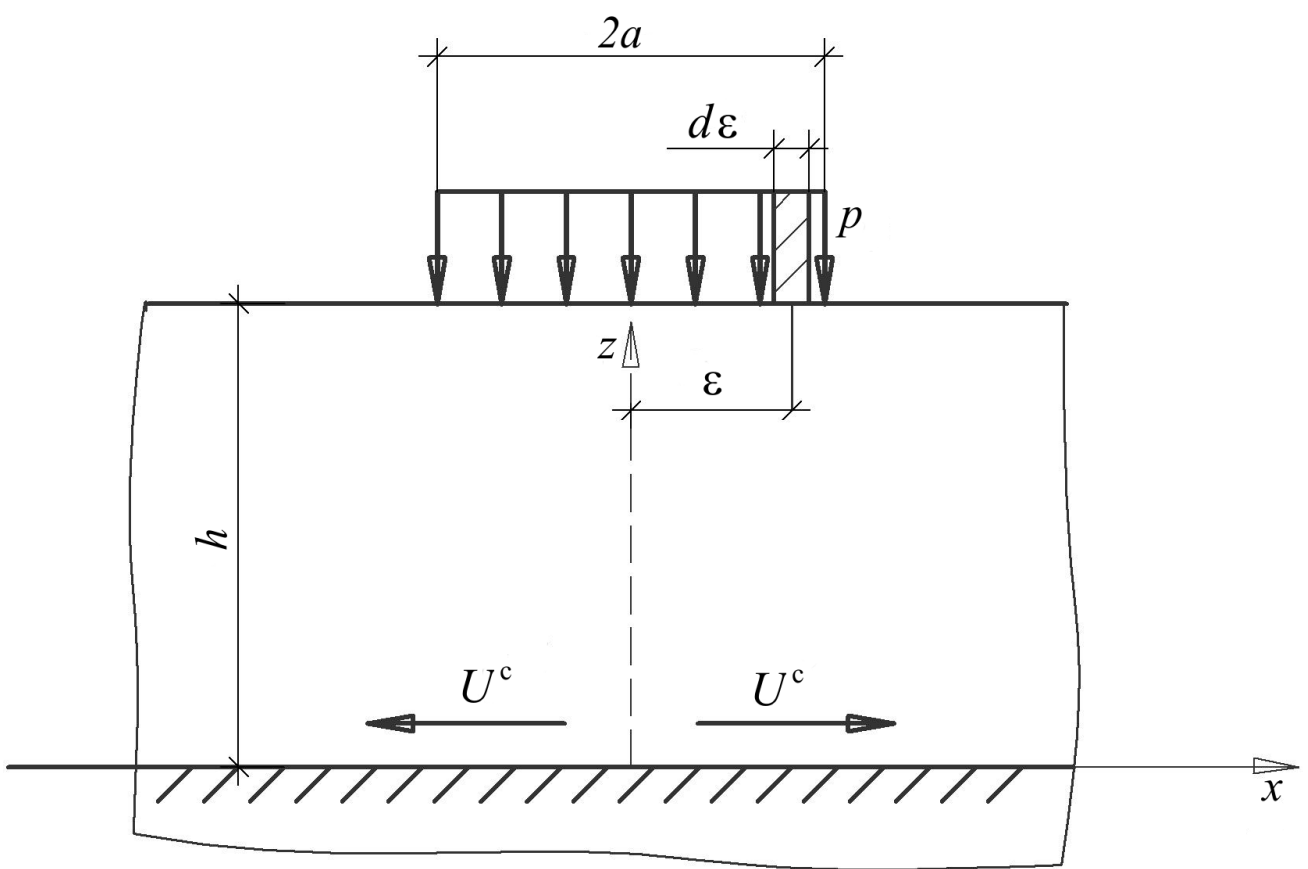

Рис. 4.18. Схема к расчету напряженно-деформированного состояния бесконечно простирающегося упругого массива ограниченной толщины на жестком основании, допускающем только горизонтальные смещения чистого сдвига, от полосовой равномерной нагрузки 
С целью определения компонентов напряжений и сдвиговых смещений запишем частные производные (4.102)

$$
\begin{gathered}
\frac{\partial \sigma}{\partial x}=\frac{p}{2 h}\left\{\cos \frac{\pi z}{2 h}\left[\frac{\operatorname{ch} \frac{\pi(x+a)}{2 h}}{\operatorname{sh}^{2} \frac{\pi(x+a)}{2 h}+\cos ^{2} \frac{\pi z}{2 h}}-\frac{\operatorname{ch} \frac{\pi(x-a)}{2 h}}{\operatorname{ch}^{2} \frac{\pi(x-a)}{2 h}+\cos ^{2} \frac{\pi z}{2 h}}\right]\right\} \\
\frac{\partial \sigma}{\partial z}=-\frac{p}{2 h}\left\{\sin \frac{\pi z}{2 h}\left[\frac{\operatorname{ch} \frac{\pi(x+a)}{2 h}}{\operatorname{sh}^{2} \frac{\pi(x+a)}{2 h}+\cos ^{2} \frac{\pi z}{2 h}}-\frac{\operatorname{sh} \frac{\pi(x-a)}{2 h}}{\operatorname{sh}^{2} \frac{\pi(x-a)}{2 h}+\cos ^{2} \frac{\pi z}{2 h}}\right]\right\} .
\end{gathered}
$$

Компоненты напряженного состояния упругого массива в соответствии с (3.39) и (4.102) будут:

$$
\begin{aligned}
& \sigma_{x}=\frac{p}{\pi}\left[\operatorname{arctg} \frac{\operatorname{sh} \frac{\pi(x+a)}{2 h}}{\cos \frac{\pi z}{2 h}}-\operatorname{arctg} \frac{\operatorname{sh} \frac{\pi(x-a)}{2 h}}{\cos \frac{\pi z}{2 h}}\right]+\frac{p}{2 h}(h-z) \times \\
& \times\left\{\sin \frac{\pi z}{2 h}\left[\frac{\operatorname{sh} \frac{\pi(x+a)}{2 h}}{\operatorname{sh}^{2} \frac{\pi(x+a)}{2 h}+\cos ^{2} \frac{\pi z}{2 h}}-\frac{\operatorname{sh} \frac{\pi(x-a)}{2 h}}{\operatorname{sh}^{2} \frac{\pi(x-a)}{2 h}+\cos ^{2} \frac{\pi z}{2 h}}\right]\right\} \\
& \sigma_{z}=\frac{p}{\pi}\left[\operatorname{arctg} \frac{\operatorname{sh} \frac{\pi(x+a)}{2 h}}{\cos \frac{\pi z}{2 h}}-\operatorname{arctg} \frac{\operatorname{sh} \frac{\pi(x-a)}{2 h}}{\cos \frac{\pi z}{2 h}}\right]+\frac{p}{2 h}(h-z) \times \\
& \times\left\{\sin \frac{\pi z}{2 h}\left[\frac{\operatorname{sh} \frac{\pi(x+a)}{2 h}}{\operatorname{sh}^{2} \frac{\pi(x+a)}{2 h}+\cos ^{2} \frac{\pi z}{2 h}}-\frac{\operatorname{sh} \frac{\pi(x-a)}{2 h}}{\operatorname{sh}^{2} \frac{\pi(x-a)}{2 h}+\cos ^{2} \frac{\pi z}{2 h}}\right]\right\}
\end{aligned}
$$




$$
\begin{gathered}
\sigma_{y}=\frac{p}{\pi}\left[\operatorname{arctg} \frac{\operatorname{sh} \frac{\pi(x+a)}{2 h}}{\cos \frac{\pi z}{2 h}}-\operatorname{arctg} \frac{\operatorname{sh} \frac{\pi(x-a)}{2 h}}{\cos \frac{\pi z}{2 h}}\right] ; \\
\tau_{x z}=\frac{P}{\pi}(h-z)\left\{\cos \frac{\pi z}{2 h}\left[\frac{\operatorname{ch} \frac{\pi(x-a)}{2 h}}{\operatorname{sh}^{2} \frac{\pi(x+a)}{2 h}+\cos ^{2} \frac{\pi z}{2 h}}-\frac{\operatorname{ch} \frac{\pi(x-a)}{2 h}}{\operatorname{sh}^{2} \frac{\pi(x-a)}{2 h}+\cos ^{2} \frac{\pi z}{2 h}}\right]\right\} .
\end{gathered}
$$

Компоненты смещений точек упругого массива от чистого формоизменения получим согласно (3.28), (3.30):

$$
\begin{aligned}
& U^{\mathrm{c}}=-K^{\mathrm{c}} \frac{\partial \sigma}{\partial x}=-K^{\mathrm{c}} \frac{p}{2 h} \times \\
& \times\left\{\cos \frac{\pi z}{2 h}\left[\frac{\operatorname{ch} \frac{\pi(x+a)}{2 h}}{\operatorname{sh}^{2} \frac{\pi(x+a)}{2 h}+\cos ^{2} \frac{\pi z}{2 h}}-\frac{\operatorname{ch} \frac{\pi(x-a)}{2 h}}{\operatorname{sh} \frac{\pi(x-a)}{2 h}+\cos ^{2} \frac{\pi z}{2 h}}\right]\right\} \\
& W^{\mathrm{c}}=-K^{\mathrm{c}} \frac{\partial \sigma}{\partial z}=K^{\mathrm{c}} \frac{p}{2 h} \times \\
& \times\left\{\sin \frac{\pi z}{2 h}\left[\frac{\operatorname{sh} \frac{\pi(x+a)}{2 h}}{\operatorname{sh}^{2} \frac{\pi(x+a)}{2 h}+\cos ^{2} \frac{\pi z}{2 h}}-\frac{\operatorname{sh} \frac{\pi(x-a)}{2 h}}{\operatorname{sh} \frac{\pi(x-a)}{2 h}+\cos ^{2} \frac{\pi z}{2 h}}\right]\right\} .
\end{aligned}
$$

Смещения точек упругого массива от объемного деформирования можно получить путем интегрирования функции (4.97) с учетом (3.17)-(3.19): 


$$
W^{0}=K^{0} \int \sigma d z=K^{0} \frac{p}{\pi} \int\left[\operatorname{arctg} \frac{\operatorname{sh} \frac{\pi(x+a)}{2 h}}{\cos \frac{\pi z}{2 h}}-\operatorname{arctg} \frac{\operatorname{sh} \frac{\pi(x-a)}{2 h}}{\cos \frac{\pi z}{2 h}}\right] d z+C
$$

где $C$ - произвольная интегрирования, которая может быть определена из граничных условий: при $z=0, \quad W^{0}=0$.

\subsection{5. Бесконечно простирающийся упругий массив на жестком основании, допускающем только вертикальные смешения}

Эта задача отличается от задачи п 4.3.4 тем, что условия контакта массива допускают вместо горизонтальных смещений от чистого формоизменения вертикальные (рис. 4.19). Граничными условиями для функции давления будут:

$$
\begin{gathered}
z=0, \frac{\partial \sigma}{\partial x}=0\left(U^{\mathrm{c}}=0\right) \\
z=h, x \neq 0, \quad \sigma=0 ; \quad z=h, x=0, \quad \sigma=\infty \\
\sigma(x, z)=\sigma(-x, z) .
\end{gathered}
$$

Функция давления, отвечающая поставленным граничным условиям, может быть получена из решения (4.94) для ограниченной ширины массива (см. рис. 4.15), в котором следует выполнить предельный переход путем $l \rightarrow \infty$. Предварительно запишем это решение в виде

$$
\sigma_{\text {огр }}=\frac{P}{2 l} \frac{2 l}{\pi} \sum_{n=1}^{n=\infty} \frac{\operatorname{sh}\left(z t_{n}\right)}{\operatorname{sh}\left(h t_{n}\right)} \cos \left(x t_{n}\right) \Delta t_{n},
$$

где

$$
t_{\mathrm{n}}=\frac{\pi(2 n-1)}{2 l}
$$


При неограниченном возрастании ширины массива $l$ суммирование заменяем интегрированием:

$$
\sigma=\lim _{l \rightarrow \infty} \sigma_{\text {огр }}=\frac{P}{\pi} \int_{0}^{\infty} \frac{\operatorname{sh}(z t)}{\operatorname{sh}(h t)} \cos (x t) d t .
$$

Согласно [29] несобственный интеграл такого вида сходится к своему пределу:

$$
\int_{0}^{\infty} \frac{\operatorname{sh} \beta x}{\operatorname{sh} \gamma x} \cos \alpha_{x} d x=\frac{\pi}{2 \gamma} \frac{\sin \frac{\pi \beta}{\gamma}}{\operatorname{ch} \frac{\pi \alpha}{\gamma}+\cos \frac{\pi \beta}{\gamma}} .
$$

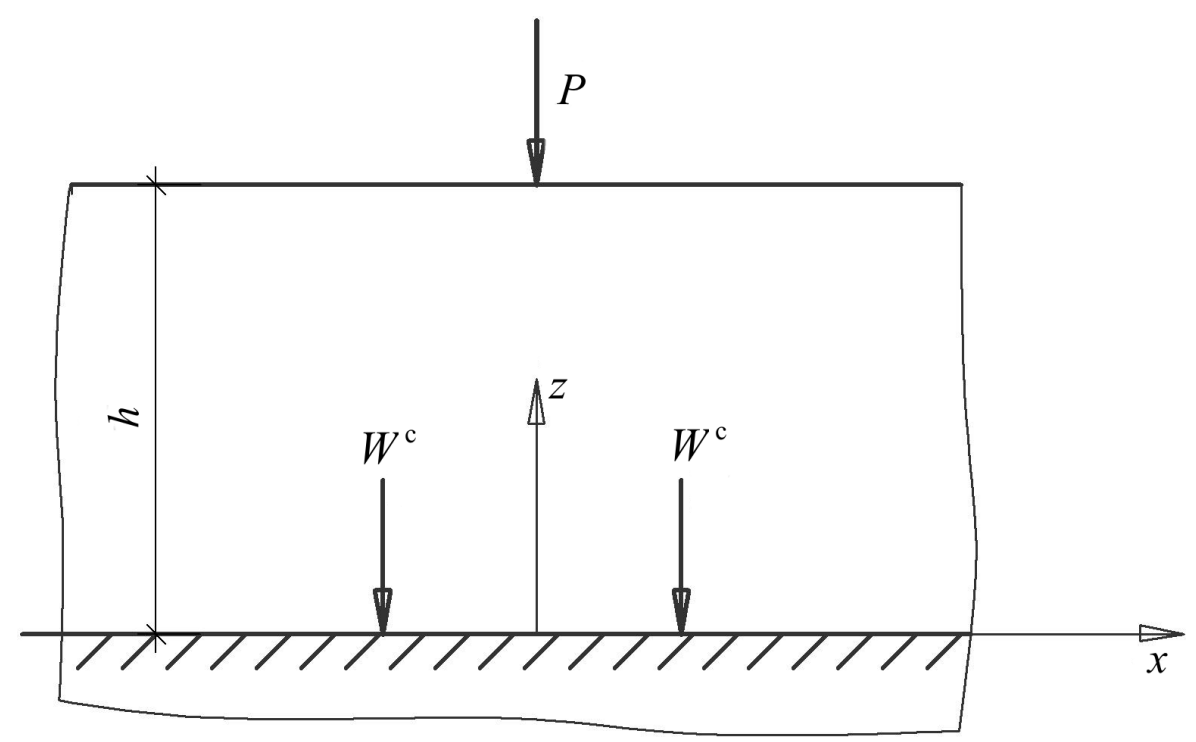

Рис. 4.19. Схема к расчету напряженно-деформированного состояния бесконечно простирающегося упругого массива ограниченной толщины на жестком основании, допускающем только вертикальные перемещения чистого сдвига, от линейно распределенной нагрузки

Выполнив соответствующую подстановку, получим искомую функцию давления:

$$
\sigma=\frac{P}{2 h} \frac{\sin \frac{\pi z}{h}}{\operatorname{ch} \frac{\pi x}{h}+\cos \frac{\pi z}{h}} .
$$


Для дальнейшего анализа решения и определения компонентов напряжений и деформаций запишем первые и вторые частные производные полученной функции давления (4.103):

первые частные производные

$$
\begin{aligned}
& \frac{\partial \sigma}{\partial x}=-\frac{P \pi}{2 h^{2}} \frac{\operatorname{sh} \frac{\pi x}{h} \sin \frac{\pi z}{h}}{\left(\operatorname{ch} \frac{\pi x}{h}+\cos \frac{\pi z}{h}\right)^{2}} ; \\
& \frac{\partial \sigma}{\partial z}=-\frac{P \pi}{2 h^{2}} \frac{1+\operatorname{ch} \frac{\pi x}{h} \cos \frac{\pi z}{h}}{\left(\operatorname{ch} \frac{\pi x}{h}+\cos \frac{\pi z}{h}\right)^{2}} ;
\end{aligned}
$$

вторые частные производные

$$
\begin{gathered}
\frac{\partial^{2} \sigma}{\partial x^{2}}=-\frac{P \pi^{2}}{2 h^{3}} \frac{\sin \frac{\pi z}{h}\left(1-\operatorname{sh}^{2} \frac{\pi x}{h}+\operatorname{ch} \frac{\pi x}{h} \cos \frac{\pi z}{h}\right)}{\left(\operatorname{ch} \frac{\pi x}{h}+\cos \frac{\pi z}{h}\right)^{3}} \\
\frac{\partial^{2} \sigma}{\partial z^{2}}=\frac{P \pi^{2}}{2 h^{3}} \frac{\sin \frac{\pi z}{h}\left(1-\operatorname{sh}^{2} \frac{\pi x}{h}+\operatorname{ch} \frac{\pi x}{h} \cos \frac{\pi z}{h}\right)}{\left(\operatorname{ch} \frac{\pi x}{h}+\cos \frac{\pi z}{h}\right)^{3}} .
\end{gathered}
$$

Можно убедиться, что функция (4.103) удовлетворяет поставленным граничным условиям и является гармонической, следовательно, она обладает потенциалом.

Подтверждением взаимной связи решений теории упругости является возможность предельного перехода от решения одной задачи к решению другой задачи. В связи с этим попробуем перейти от функции давления (4.103) в данной задаче к функции давления при плоском деформировании полупространства равномерно распределенной вдоль бесконечной линии нормальной нагрузкой (4.59). В целях 
удобства преобразований мысленно перенесем начало координат на рис. 4.19 вертикально вверх, в точку приложения нагрузки. В новой координатной системе функция (4.103) запишется в виде

$$
\sigma=\frac{P}{2 h} \frac{\sin \frac{\pi(h-z)}{h}}{\operatorname{ch} \frac{\pi x}{h}+\cos \frac{\pi(h-z)}{h}},
$$

где координата $z$ направлена вниз, а область ее изменения будет $h \geq z \geq 0$.

Для перехода к функции давления в полупространстве от аналогичной нагрузки будем неограниченно увеличивать толщину упругого слоя $h \rightarrow \infty$ :

$$
\sigma=\lim _{h \rightarrow \infty} \frac{P}{2 h} \frac{\sin \frac{\pi(h-z)}{h}}{\operatorname{ch} \frac{\pi x}{h}+\cos \frac{\pi(h-z)}{h}}=\frac{0}{0} .
$$

Для раскрытия неопределенности воспользуемся правилом Лопиталя и запишем первые производные по $h$ в числителе и знаменателе:

$$
\sigma=\lim _{h \rightarrow \infty} \frac{P}{2 h} \frac{\cos \frac{\pi(h-z)}{h}\left(\frac{\pi z}{h^{2}}\right)}{\operatorname{sh} \frac{\pi x}{h}\left(-\frac{\pi z}{h^{2}}\right)-\sin \frac{\pi(h-z)}{h}\left(\frac{\pi z}{h^{2}}\right)}=\frac{0}{0} .
$$

Поскольку неопределенность не раскрыта, записываем в числителе и знаменателе вторые производные по $h$ :

$$
\begin{aligned}
& \sigma=\lim _{h \rightarrow \infty} \frac{P}{2 h} \times \\
& \times \frac{-\sin \frac{\pi(h-z)}{h}\left(\frac{\pi z}{h^{2}}\right)^{2}-\cos \frac{\pi(h-z)}{h} \frac{2 \pi z}{h^{3}}}{\operatorname{ch} \frac{\pi x}{h}\left(-\frac{\pi z}{h^{2}}\right)^{2}+\operatorname{sh} \frac{\pi x}{h}\left(-\frac{\pi z}{h^{3}}\right)-\cos \frac{\pi(h-z)}{h}\left(-\frac{\pi z}{h^{2}}\right)^{2}+\sin \frac{\pi(h-z)}{h} \frac{4 \pi z}{h^{3}}}=
\end{aligned}
$$




$$
=\lim _{h \rightarrow \infty} \frac{P}{2 h} \frac{2 \frac{\pi z}{h^{3}}}{\frac{\pi^{2}}{h^{4}}\left(x^{2}+z^{2}\right)}=\frac{P}{\pi} \frac{z}{x^{2}+z^{2}} .
$$

Таким образом, путем предельного перехода от (4.103) мы получили функцию давления при нагружении поверхности полупространства нагрузкой, равномерно распределенной вдоль бесконечной линии (4.59), что подтверждает взаимосвязь решений и является их контролем.

Компоненты напряженного состояния в данной задаче в соответствии с условиями (3.39) определяются зависимостями

$$
\begin{gathered}
\sigma_{x}=\frac{P}{2 h} \frac{\sin \frac{\pi z}{h}}{\operatorname{ch} \frac{\pi x}{h}+\cos \frac{\pi z}{h}}-(h-z) \frac{P \pi}{2 h^{2}} \frac{1+\operatorname{ch} \frac{\pi x}{h} \cos \frac{\pi z}{h}}{\left(\operatorname{ch} \frac{\pi x}{h}+\cos \frac{\pi z}{h}\right)^{2}} \\
\sigma_{z}=\frac{P}{2 h} \frac{\sin \frac{\pi z}{h}}{\operatorname{ch} \frac{\pi x}{h}+\cos \frac{\pi z}{h}}+(h-z) \frac{P \pi}{2 h^{2}} \frac{1+\operatorname{ch} \frac{\pi x}{h} \cos \frac{\pi z}{h}}{\left(\operatorname{ch} \frac{\pi x}{h}+\cos \frac{\pi z}{h}\right)^{2}} \\
\sigma_{y}=\frac{P}{2 h} \frac{\sin \frac{\pi z}{h}}{\operatorname{ch} \frac{\pi x}{h}+\cos \frac{\pi z}{h}} ; \\
\tau_{z x}=(h-z) \frac{P \pi}{2 h^{2}} \frac{\operatorname{sh} \frac{\pi x}{h} \sin \frac{\pi z}{h}}{\left(\operatorname{ch} \frac{\pi x}{h}+\cos \frac{\pi z}{h}\right)^{2}} .
\end{gathered}
$$

Чтобы убедиться, выполняется ли условие общего равновесия в данной задаче, проинтегрируем компонент $\sigma_{z}$ на контакте упругого массива с жестким основанием, в результате чего мы должны получить внешнюю нагрузку $P(\mathrm{H} / \mathrm{M})$. Выполним эту операцию. 
Компонент $\sigma_{z}(4.104)$ для случая $z=0$ принимает вид

$$
\underset{\substack{z \\ z=0}}{\sigma_{2}}=\frac{P \pi}{2 h} \frac{1+\operatorname{ch} \frac{\pi x}{h}}{\left(1+\operatorname{ch} \frac{\pi x}{h}\right)^{2}}=\frac{P \pi}{2 h\left(1+\operatorname{ch} \frac{\pi x}{h}\right)} .
$$

Составляем уравнение равновесия

$$
\begin{gathered}
2 \int_{0}^{\infty} \sigma_{z} d x-P=0 ; \\
\frac{P \pi}{h} \int_{0}^{\infty} \frac{d x}{1+\operatorname{ch} \frac{\pi x}{h}}-P=0, \\
P \int_{0}^{\infty} \frac{d\left[\frac{\pi x}{h}\right]}{1+\operatorname{ch} \frac{\pi x}{h}}-P=\left.P \operatorname{th} \frac{\pi x}{2 h}\right|_{0} ^{\infty}-P=P \frac{e^{\pi x / h}-1}{e^{\pi x / h}+1}-P=P(1-0)-P=0 .
\end{gathered}
$$

Очевидно, что условие общего равновесия в задаче выполняется. Запишем теперь компоненты смещений точек упругого массива от чистого формоизменения в рассматриваемой задаче:

$$
\begin{gathered}
U^{\mathrm{c}}=-K^{\mathrm{c}} \frac{\partial \sigma}{\partial x}=\frac{P \pi}{2 h^{2}} \frac{\operatorname{sh} \frac{\pi x}{h} \sin \frac{\pi z}{h}}{\left(\operatorname{ch} \frac{\pi x}{h}+\cos \frac{\pi z}{h}\right)^{2}} ; \\
W^{\mathrm{c}}=-K^{\mathrm{c}} \frac{\partial \sigma}{\partial z}=-\frac{P \pi}{2 h^{2}} \frac{1+\operatorname{ch} \frac{\pi x}{h} \sin \frac{\pi z}{h}}{\left(\operatorname{ch} \frac{\pi x}{h}+\cos \frac{\pi z}{h}\right)^{2}} ;
\end{gathered}
$$


Вертикальные перемещения точек массива от изменения его плотности определяются интегрированием (4.103) с учетом зависимостей (3.17)-(3.19):

$$
\begin{gathered}
W^{0}=K^{0} \int \sigma d z=K^{0} \frac{P}{2 h} \int \frac{\sin \frac{\pi z}{h} d z}{\operatorname{ch} \frac{\pi x}{h}+\cos \frac{\pi z}{h}}, \\
W^{0}=K^{0} \frac{P}{2 h} \ln \left|\operatorname{ch} \frac{\pi x}{h}+\cos \frac{\pi z}{h}\right|+C .
\end{gathered}
$$

Здесь $C$ - постоянная интегрирования, которая определяется из граничных условий: при $z=0$ вертикальное перемещение от изменения плотности отсутствует, так как на этом уровне $\sigma=0$, поэтому

$$
C=-K^{0} \frac{P}{2 \pi} \ln \left|\operatorname{ch} \frac{\pi x}{h}+1\right|
$$

и тогда

$$
W^{0}=-K^{0} \frac{P}{2 \pi} \ln \left|\frac{\operatorname{ch} \frac{\pi x}{h}+1}{\operatorname{ch} \frac{\pi x}{h}+\cos \frac{\pi z}{h}}\right| .
$$

В том случае, когда на поверхности бесконечно простирающегося упругого массива ограниченной толщины в данной задаче будет действовать равномерно распределенная нагрузка $p\left(\mathrm{H} / \mathrm{M}^{2}\right)$ по полосе бесконечной длины и постоянной ширины $2 a$, задача определения функции давления решается путем интегрирования (4.103) согласно рис. 4.20:

$$
\sigma=\frac{P}{2 h} \int_{-a}^{a} \frac{\sin \frac{\pi x}{h} d(x-\varepsilon)}{\operatorname{ch} \frac{\pi(x-\varepsilon)}{h}+\cos \frac{\pi z}{h}}
$$


После интегрирования согласно [30] и подстановки пределов получим:

$\sigma=\frac{p}{2 \pi}\left\{\frac{1}{\sin \frac{\pi z}{h}}\left[\arcsin \frac{1+\operatorname{ch} \frac{\pi(x+a)}{h} \cos \frac{\pi z}{h}}{\operatorname{ch} \frac{\pi(x+a)}{h} \cos \frac{\pi z}{h}}-\right.\right.$

$$
\left.\left.-\arcsin \frac{1+\operatorname{ch} \frac{\pi(x-a)}{h} \cos \frac{\pi z}{h}}{\operatorname{ch} \frac{\pi(x-a)}{h} \cos \frac{\pi z}{h}}\right]\right\} \text {. }
$$

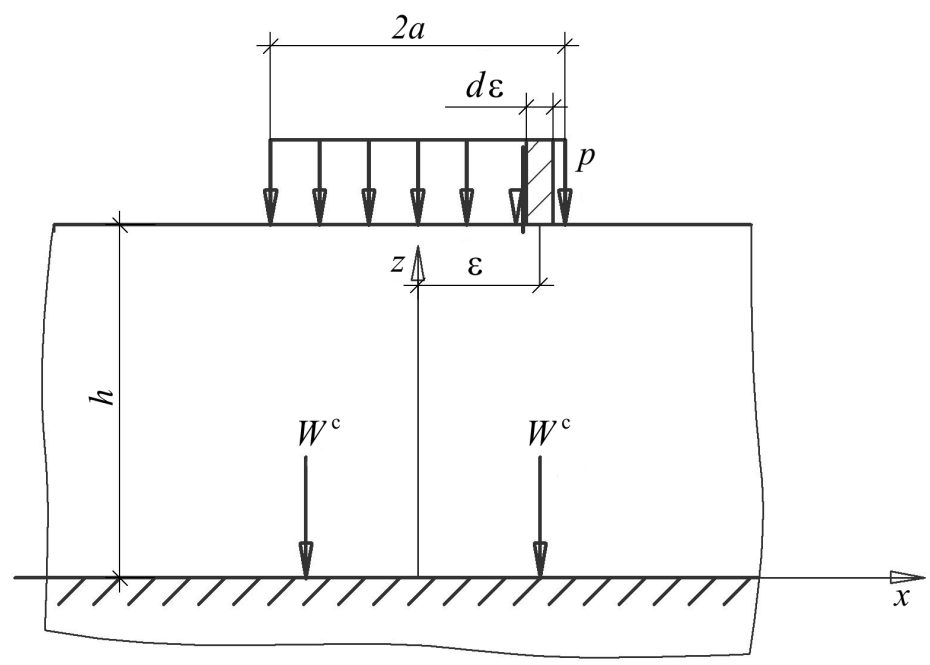

Рис. 4.20. Схема к расчету напряженно-деформированного состояния бесконечно простирающегося упругого массива ограниченной толщины на жестком основании, допускающем только вертикальные смещения чистого сдвига, от полосовой равномерной нагрузки

Для определения компонентов напряжений и перемещений от чистого формоизменения запишем частные производные (4.105):

$$
\frac{\partial \sigma}{\partial x}=-\frac{p}{2 h}\left[\frac{1}{\operatorname{ch} \frac{\pi(x+a)}{h}+\cos \frac{\pi z}{h}}-\frac{1}{\operatorname{ch} \frac{\pi(x-a)}{h}+\cos \frac{\pi z}{h}}\right]
$$




$$
\begin{aligned}
\frac{\partial \sigma}{\partial x}=\frac{p}{2 h}\left\{\ln \left|\operatorname{tg} \frac{\pi z}{2 h}\right| \times\right. & \\
\times & {\left[\arcsin \frac{1+\operatorname{ch} \frac{\pi(x+a)}{h} \cos \frac{\pi z}{h}}{\operatorname{ch} \frac{\pi(x+a)}{h}+\cos \frac{\pi z}{h}}-\arcsin \frac{1+\operatorname{ch} \frac{\pi(x-a)}{h} \cos \frac{\pi z}{h}}{\left.\operatorname{ch} \frac{\pi(x-a)}{h}-\cos \frac{\pi z}{h}\right]-}\right.} \\
& \left.-\frac{1}{\sin \frac{\pi z}{h}}\left[\frac{\operatorname{sh} \frac{\pi(x+a)}{h}}{\operatorname{ch} \frac{\pi(x+a)}{h}+\cos \frac{\pi z}{h}}-\frac{\operatorname{sh} \frac{\pi(x-a)}{h}}{\operatorname{ch} \frac{\pi(x-a)}{h}+\cos \frac{\pi z}{h}}\right]\right\} .
\end{aligned}
$$

В формулах (4.105)-(4.107) перед членами, содержащими положительное значение $(x+a)$ либо $(x-a)$, следует поменять знак на противоположный.

Обозначив содержимое внешних скобок в (4.105)-(4.107) буквами соответственно $A, B$ и $D$, записываем компоненты напряженного состояния:

$$
\begin{gathered}
\sigma_{x}=\frac{p}{2 \pi} A+\frac{p}{2 h}(h-z) D ; \\
\sigma_{z}=\frac{p}{2 \pi} A-\frac{p}{2 h}(h-z) D ; \\
\tau_{z x}=\frac{p}{2 h}(h-z) B .
\end{gathered}
$$

Смещение точек среды от объемного деформирования можно получить интегрированием функции (4.105):

$$
W^{0}=\frac{p}{2 \pi} \int A d z+C
$$

где произвольная интегрирования $C$ определяется из граничных условий. 


\subsection{6. Бесконечно простирающийся упругий массив на жестком основании, допускающем частично горизонтальные и вертикальные смещения}

Выше были рассмотрены решения по определению напряженнодеформированного состояния бесконечно простирающегося упругого массива конечной толщины на жестком основании при двух предельных значениях граничных условий на контакте: основание допускает только горизонтальные смещения чистого сдвига (п. 4.3.4) и основание допускает только вертикальные смещения чистого сдвига (п. 4.3.5). В том же случае, когда основание допускает частично горизонтальные и вертикальные смещения, решение можно получить путем комбинирования двух упомянутых решений, подобно тому как это было сделано в п. 4.3.3. Запишем такое решение для случая равномерно распределенной линейной нагрузки интенсивностью $P(\mathrm{H} / \mathrm{M})$.

Функция давления

$$
\sigma=\frac{P}{2 h}\left[m^{\Gamma} \frac{\operatorname{ch} \frac{\pi x}{2 h} \cos \frac{\pi z}{2 h}}{\operatorname{sh}^{2} \frac{\pi x}{2 h}+\cos ^{2} \frac{\pi z}{2 h}}+m^{\mathrm{B}} \frac{\operatorname{sh} \frac{\pi x}{h} \sin \frac{\pi z}{h}}{\operatorname{ch}^{2} \frac{\pi x}{h}+\cos ^{2} \frac{\pi z}{h}}\right]
$$

компоненты напряжений

$$
\begin{aligned}
\sigma_{x}=\frac{P}{2 h}\left\{m ^ { \Gamma } \left[\frac{\operatorname{ch} \frac{\pi x}{2 h} \cos \frac{\pi z}{2 h}}{\operatorname{sh}^{2} \frac{\pi x}{2 h}+\cos ^{2} \frac{\pi z}{2 h}-\frac{\pi}{2 h}(h-z) \times}\right.\right. \\
\\
\left.\quad \times \frac{\operatorname{ch} \frac{\pi x}{2 h} \sin \frac{\pi z}{2 h}\left(\operatorname{sh}^{2} \frac{\pi x}{2 h}-\cos ^{2} \frac{\pi z}{2 h}\right)}{\left(\operatorname{sh}^{2} \frac{\pi x}{2 h}+\cos ^{2} \frac{\pi z}{2 h}\right)^{2}}\right]+
\end{aligned}
$$




$$
\begin{aligned}
& \left.+m^{\mathrm{B}}\left[\frac{\sin \frac{\pi z}{h}}{\operatorname{ch} \frac{\pi x}{2 h}+\cos \frac{\pi z}{h}}-\frac{\pi}{h}(h-z) \frac{1+\operatorname{ch} \frac{\pi x}{h} \cos \frac{\pi z}{h}}{\left(\operatorname{ch} \frac{\pi x}{h}+\cos \frac{\pi z}{h}\right)^{2}}\right]\right\}, \\
& \sigma_{z}=\frac{P}{2 h}\left\{m ^ { \mathrm { r } } \left[\frac{\operatorname{ch} \frac{\pi x}{2 h} \cos \frac{\pi z}{2 h}}{\operatorname{sh}^{2} \frac{\pi x}{2 h}+\cos ^{2} \frac{\pi z}{2 h}}+\frac{\pi}{2 h}(h-z) \times\right.\right. \\
& \left.\times \frac{\operatorname{ch} \frac{\pi x}{2 h} \sin \frac{\pi z}{2 h}\left(\operatorname{sh}^{2} \frac{\pi x}{2 h}-\cos ^{2} \frac{\pi z}{2 h}\right)}{\left(\operatorname{sh}^{2} \frac{\pi x}{2 h}+\cos ^{2} \frac{\pi z}{2 h}\right)^{2}}\right]+ \\
& \left.+m^{\mathrm{B}}\left[\frac{\sin \frac{\pi z}{h}}{\operatorname{ch} \frac{\pi x}{2 h}+\cos \frac{\pi z}{h}}+\frac{\pi}{h}(h-z) \frac{1+\operatorname{ch} \frac{\pi x}{h} \cos \frac{\pi z}{h}}{\left(\operatorname{ch} \frac{\pi x}{h}+\cos \frac{\pi z}{h}\right)^{2}}\right]\right\}, \\
& \tau_{x z}=-\frac{P \pi}{h^{2}}(h-z)\left[\frac{m^{\mathrm{r}}}{2} \frac{\operatorname{sh} \frac{\pi x}{2 h} \cos \frac{\pi z}{2 h}\left(\operatorname{ch}^{2} \frac{\pi x}{2 h}-\sin \frac{\pi z}{2 h}\right)}{\left(\operatorname{sh}^{2} \frac{\pi x}{h}+\cos \frac{\pi z}{2 h}\right)^{2}}-\right. \\
& \left.-m^{\mathrm{B}} \frac{\operatorname{sh} \frac{\pi x}{h} \sin \frac{\pi z}{h}}{\operatorname{ch}^{2} \frac{\pi x}{h}+\cos \frac{\pi z}{h}}\right]
\end{aligned}
$$


компоненты смещений чистого сдвига

$$
\begin{gathered}
U^{\mathrm{c}}=K^{\mathrm{c}} \frac{P \pi}{2 h^{2}}\left[\frac{m^{\mathrm{\Gamma}}}{2} \frac{\operatorname{sh} \frac{\pi x}{2 h} \cos \frac{\pi z}{2 h}\left(\operatorname{ch}^{2} \frac{\pi x}{2 h}-\sin ^{2} \frac{\pi z}{2 h}\right)}{\left(\operatorname{sh}^{2} \frac{\pi x}{2 h}+\cos ^{2} \frac{\pi z}{2 h}\right)^{2}}-m^{\mathrm{B}} \frac{\operatorname{sh} \frac{\pi x}{h} \sin \frac{\pi z}{h}}{\operatorname{ch} \frac{\pi x}{h}+\cos \frac{\pi z}{h}}\right], \\
W^{\mathrm{c}}=-K^{\mathrm{c}} \frac{P \pi}{2 h^{2}}\left[\frac{m^{\mathrm{\Gamma}}}{2} \frac{\operatorname{ch} \frac{\pi x}{2 h} \sin \frac{\pi z}{2 h}\left(\operatorname{sh}^{2} \frac{\pi x}{2 h}-\cos ^{2} \frac{\pi z}{2 h}\right)}{\left(\operatorname{sh}^{2} \frac{\pi x}{2 h}+\cos ^{2} \frac{\pi z}{2 h}\right)^{2}}-\right. \\
\left.-m^{\mathrm{B}} \frac{1+\operatorname{ch} \frac{\pi x}{h} \sin \frac{\pi z}{h}}{\left(\operatorname{ch} \frac{\pi x}{h}+\cos \frac{\pi z}{h}\right)^{2}}\right],
\end{gathered}
$$

вертикальные смещения от изменения плотности упругого массива

$$
W^{0}=-K^{0} \frac{P}{\pi}\left(m^{\mathrm{\Gamma}} \ln \frac{\operatorname{ch} \frac{\pi x}{2 h}+\sin \frac{\pi z}{2 h}}{\operatorname{ch} \frac{\pi x}{2 h}-\sin \frac{\pi z}{2 h}}+\frac{m^{\mathrm{B}}}{2} \ln \left|\frac{\operatorname{ch} \frac{\pi x}{h}+1}{\operatorname{ch} \frac{\pi x}{h}+\cos \frac{\pi z}{h}}\right|\right) .
$$




\section{ЗАКЛЮЧЕНИЕ}

Руководствуясь предложенными физическими зависимостями для упругих массивов и описанной выше последовательностью действий при определении их напряженно-деформированного состояния, получены решения ряда задач, имеющих различные граничные условия. В этот ряд входят как задачи, которые имели решения в прежней постановке, с использованием системы физических зависимостей в виде обобщенного закона Гука с присущими им недостатками, но решены теперь по-новому, так и задачи, решения которых не были известны в традиционной постановке. Среди последних важное место занимает общая задача по загружению полупространства наклонно действующей к его поверхности сосредоточенной силой. Ее решение можно интегрировать для любой распределенной нагрузки. Для случая действия в этой задаче сосредоточенной силы по нормали к поверхности полупространства будем иметь ее частный случай, относящийся к первой части решенных задач.

Ко второй части решенных задач также относятся задачи плоского деформирования бесконечно простирающегося упругого массива ограниченной толщины нормальной к его поверхности линейной и полосовой нагрузкой, а также другие задачи.

Таким образом, применение предложенных физических зависимостей расширяет круг аналитически решаемых задач, а сами решения выглядят проще по сравнению с теми, которые были известны в традиционной постановке. И, что самое главное, во всех получаемых решениях строго выполняются законы механики. 


\section{СПИСОКИСПОЛЬЗОВАННОЙ ЛИТЕРАТУРЫ}

1. Безухов, Н. И. Основы теории упругости, пластичности и ползучести [Текст] / Н. И. Безухов. - М.: Высшая шк., 1961. - 537 с.

2. Лейбензон, Л. С. Курс теории упругости [Текст] / Л. С. Лейбензон. - М.; Л.: ОГИЗ - Гостехиздат, 1947. - 464 с.

3. Филоненко-Бородич, М. М. Теория упругости [Текст] / М. М. ФилоненкоБородич. - М.: Физматгиз, 1959. - 364 с.

4. Тимошенко, С. П. Теория упругости [Текст]: пер. с. англ. / С. П. Тимошенко, Дж. Гудьер. - М.: Наука, 1975. - 576 с.

5. Ляв, А. Математическая теория упругости [Текст]: пер. с 4-го англ. издания / А. Ляв. - М.; Л.: Изд-во НКТП СССР, 1935. - 674 с.

6. Boussinesq, J. Application des potentiels a l'equilibre et du movement des solides elastiques [Text] / J. Boussinesq. - Paris, 1885.

7. Flamant, Comptes rendus, t. 114, Paris, 1892.

8. Флорин, В. А. Основы механики грунтов [Текст].: в 2 т. / В. А. Флорин // Л.; М.: Гос. изд-во лит-ры по строительству, архитектуре и строительным материалам. 1959. - т. 1. - 359 с.

9. Цытович, Н. А. Механика грунтов [Текст] / Н. А. Цытович. - М.: Гос. издво лит-ры по строительству, архитектуре и строительным материалам, 1963. $-636 \mathrm{c}$.

10. Kelvin, Cambridge and Dublin Math. J., 1848.

11. Жемочкин, Б. Н. Теория упругости [Текст] / Б. Н. Жемочкин. - М.: Гос. изд-во лит-ры по строительству и архитектуре, 1957. - 256 с.

12. Бадалаха, И. К. Определение напряженно-деформированного состояния упругих массивов путем выделения объемных и сдвиговых деформаций [Текст] / И. К. Бадалаха // Межведомственный сб. науч. тр. / Ин-т геотехн. механики НАН Украины. - Д.: Поліграфіст, 2000 - Вып. 18. - С. 119-127.

13. Гольдштейн, М. Н. Механика грунтов, основания и фундаменты [Текст] / М. Н. Гольдштейн, А. А. Царьков, И. И. Черкасов. - М.: Транспорт, 1981. $320 \mathrm{c}$.

14. Мышкис, А. Д. Лекции по высшей математике [Текст] / А. Д. Мышкис. М.: Наука. Глав. ред. физ.-мат. лит-ры, 1967. - 640 с.

15. Овчинников, П. П. Вища математика [Текст]: в 2 ч. / П. П. Овчинников, Ф. П. Яремчук, В. М. Михайленко. - К.: Техніка, 2003. - Ч. 1. - 600 с. 
16. Крылов, А. Н. Собрание трудов [Текст]: в 11 т. / А. Н. Крылов. - М.; Л.: Изд-во АН СССР, 1951. - Т. 1, ч. 2. - 323 с.

17. Жуковский, Н. Е. Собрание сочинений [Текст]: в 7 т. / Н. Е. Жуковский. М.; Л.: Гостехиздат 1948. - Т. 2. - 764 с.

18. Павловский, Н. Н. Собрание сочинений [Текст]: в 2 т. / Н. Н. Павловский. М.; Л.: Изд-во АН СССР, 1956. - Т. 2. - 771 с.

19. Бадалаха, И. К. Постановка и решение задач теории упругости с использованием потенциала [Текст] / И. К. Бадалаха // Будівництво: зб. наук. пр. Дніпропетр. нац. ун-ту залізн. трансп. - 1999. - Вип. 6. - С. 173-184.

20. Бадалаха, І. К. Рішення задач теорії пружності з роздільним визначенням чистих і об’ємних деформацій [Текст] / I. К. Бадалаха // Тези 69-ї Міжнародної науково-техн. конф. «Проблеми та перспективи розвитку залізничного транспорту» 21-22 травня 2009 р., Дніпропетровськ. - Д.: Вид-во Дніпропетр. нац. ун-ту залізн. трансп. акад. В. Лазаряна, 2009. C. $184-185$.

21. Бадалаха, И. К. Решение задач теории упругости с раздельным определением чистых и объемных деформаций [Текст] / И. К. Бадалаха // Зб. наук. пр. Дніпропетр. нац. ун-ту залізн. трансп. ім. акад. В. Лазаряна. - 2009. Вип. 27. - С. 154-159.

22. Двайт, Г. Б. Таблицы интегралов и другие математические формулы [Текст] / Г. Б. Двайт. - М.: Наука, 1977. - 224 с.

23. Бадалаха, И. К. Напряженно-деформированное состояние упругого полупространства от погонной линейной нагрузки, действующей на ограниченном и неограниченном протяжении его поверхности [Текст] / И. К. Бадалаха // Зб. наук. пр. Дніпропетр. нац. ун-ту залізн. трансп. ім. акад. В. Лазаряна. - 2009. - Вип. 26. - С. 98-102.

24. Мачерет, Я. А. Распределение мгновенных напоров и давлений в грунтовой массе, вызванных мгновенной нагрузкой [Текст] / Я. А. Мачерет // Тр. ВИОС. Основания и фундаменты. Сб. 4: Вибрации оснований и фундаментов. - М., 1934. - С. 64-123.

25. Бадалаха, И. К. Напряженно-деформированное состояние упругого полупространства, нагруженного равномерно распределенной нагрузкой по площади прямоугольника и по бесконечной полосе [Текст] / И. К. Бадалаха // Зб. наук. пр. Дніпропетр. нац. ун-ту залізн. трансп. ім. акад. В. Лазаряна. - 2009. - Вип. 28. - С. 82-89.

26. Короткин, В. Г. Объемная задача для упруго-изотропного полупространства [Текст] / В. Г. Короткин // Сб. 4, изыскательский выпуск / НКТП СССР, Главгидроэнергострой. Государственный всесоюзный трест по изысканиям и проектированию гидроэлектростанций и гидроэнергоузлов, Гидроэнергопроект, Ленинградское отделение. - 1938. - С. 52-85.

27. Бадалаха, И. К. Влияние гидродинамических факторов на устойчивость оснований и сооружений из насыпных грунтов [Текст]: дис ... канд. техн. наук / И. К. Бадалаха. - Д., 1980. - 199 с. 
28. Бадалаха, И. К. Напряженно-деформированное состояние бесконечно длинных упругих массивов различной ширины и ограниченной толщины на жестком основании рои их плоском деформировании [Текст] / И. К. Бадалаха // Зб. наук. пр. Дніпропетр. нац. ун-ту залізн. трансп. ім. акад. В. Лазаряна. - 2010. - Вип. 31. - С. 161-172.

29. Градштейн, И. С. Таблицы интегралов, сумм, рядов и произведений [Текст] / И. С. Градштейн, И. М. Рыжик. - М.: Наука, 1971. - 1108 с. 
Для нотаток 
УДК 624.044

Физические зависимости упругих массивов [Текст]: монография / И. К. Бадалаха. - Д.: Изд-во Днепропетр. нац. ун-та ж.-д. трансп. им. акад. В. Лазаряна, 2012. - 197 с.

ISBN 978-966-8471-51-3

Деформації пружного середовища поділяються на два види відповідно до їх походження: об'ємні й чистої формозміни. Кожен вид деформації підпорядковується своїй, окремій, залежності, тому вони визначаються окремо.

Результати виконаної роботи можуть бути використані спеціалістами в галузі фізики й механіки деформівних твердих тіл, у тому числі грунтів, викладачами, аспірантами й студентами вищих навчальних закладів, проектувальниками для розрахунку основ споруд.

Наукове видання

Бадалаха Іван Кузьмич

\title{
Фізичні залежності пружних масивів
}

\author{
Монографія \\ (Російською мовою)
}

Редактор О. О. Котова
Комп’ютерна верстка О. М. Гончаренко

Формат $60 \times 84$ 1/16. УМ. друк. арк. 11,91. Обл.-вид. арк. 10,50.

Тираж 300 пр. Зам. № ШШ

Видавництво Дніпропетровського національного університету залізничного транспорту імені академіка В. Лазаряна Свідоцтво суб’єкта видавничої справи ДК № 1315 від 31.03.2003 р.

Адреса видавництва та дільниці оперативної поліграфії: 49010, м. Дніпропетровськ, вул. Лазаряна, 2. 